\title{
SYNTHESIS OF A CFD BENCHMARK EXERCISE BASED ON A TEST IN THE PANDA FACILITY ADDRESSING THE STRATIFICATION EROSION BY A VERTICAL JET IN PRESENCE OF A FLOW OBSTRUCTION
}

\author{
Michele Andreani*(1) $^{*}$, Avinash J Gaikwad ${ }^{(2)}$, Sunil Ganju ${ }^{(3)}$, Bhuvaneshwar Gera ${ }^{(3)}$, \\ Sergey Grigoryev ${ }^{(4)}$, Luis Enrique Herranz ${ }^{(5)}$, Risto Huhtanen ${ }^{(6)}$, \\ Vivek Kale $^{(2)}$, Anton Kanaev ${ }^{(4)}$, Ralf Kapulla ${ }^{(1)}$,Stephan Kelm ${ }^{(7)}$, \\ Jongtae Kim $^{(8)}$, Takeshi Nishimura ${ }^{(9)}$, Domenico Paladino ${ }^{(1)}$, \\ Sidharth Paranjape ${ }^{(1)}$,Berthold Schramm ${ }^{(10)}$, Medhat Sharabi ${ }^{(1)}$, \\ Feng Shen ${ }^{(11)}$, Bai Wei ${ }^{(11)}$, Daqiang Yan ${ }^{(11)}$, Rongjin Zhang ${ }^{(11)}$ \\ (1) Paul Scherrer Institut (PSI), Switzerland \\ (2) Atomic Energy Regulatory Board (AERB), India \\ (3) Bhabha Atomic Research Center (BARC), India \\ (4) Nuclear Safety Institute, IBRAE, Russian Federation \\ (5) Centro de Investigaciones Energéticas, Medioambientales y Tecnológicas (CIEMAT), Spain \\ (6) VTT Technical Research Centre of Finland Ltd, Finland \\ (7) Forschungszentrum Jülich (FZJ), Germany \\ (8) Korea Atomic Energy Research Institute (KAERI), Korea \\ (9) Regulatory Standard and Research Department, \\ Secretariat of Nuclear Regulation Authority (S/NRA/R), Japan \\ (10) Gesellschaft für Anlagen- und Reaktorsicherheit (GRS), Germany \\ (11) State Power Investment Corporation Research Institute (SPICRI), People's Republic of China
}

* Corresponding author: Michele Andreani

Laboratory for Scientific Computing and Modelling

Paul Scherrer Institut, Forschungsstrasse 111, 5232 Villigen PSI, Switzerland

Tel.: ++41 563102687

Fax: ++41563104481

E-Mail: michele.andreani@psi.ch 


\begin{abstract}
The benchmark exercise discussed in this paper was conducted within the OECD/NEA project HYMERES. The specific experiment in the PANDA facility chosen for the present benchmark addresses the stratification erosion induced by a vertical steam jet, which originates from the exit of a circular pipe located below the bottom of the helium-rich layer. The mixing is somewhat slowed down by a small circular plate above the jet source. The exercise consisted of a blind phase, and an open phase. Two sets of blind simulations were requested: one set obtained using a "common model", and a second set produced by a "best estimate" model. For the "common model", a list of recommendations was given, whereas for the "best estimate" model, each participant was free to choose the modelling approach. The submitted results for the erosion times were in a large band, and especially the large differences in the results with the "common model" were not expected. The results of the best estimate simulations showed that the combination of mesh and modelling approach can lead to a wide spread of results. The most important difficulty in interpreting the results and finding the reason of the large deviations was the lack of information on the velocity field downstream of the obstruction. Therefore, for the open phase extended data from auxiliary, "zero" tests (for similar conditions but without helium layer) were provided to the participants to permit a more basic validation of their models, using a "multi-step approach". The step-by-step validation permitted some progress with respect to some of the items identified in the blind benchmark. However, large discrepancies with data in the final analyses of the test are observed, which cannot be easily attributed to specific model deficiencies or insufficient detail of the mesh. These results raised some questions in relation to best practice guidelines for the use of CFD codes for containment analysis and indicated needs for further CFD-grade experiments.
\end{abstract}

Keywords: Containment, stratification erosion, buoyant jet, benchmark, PANDA 


\section{Highlights:}

- A benchmark on gas stratification erosion was conducted using a test in the PANDA facility

- A large spread of results was observed in the blind calculations.

- The open phase was multi-step, using complementary tests with enhanced information on velocity field

- Progress was achieved, but several contributions still exhibited large discrepancies

- Results are strongly affected by the modeling of radiation heat transfer from and within the steam 


\section{INTRODUCTION}

Hydrogen generated during a (C) stulated) severe accident with core degradation is a major safety issue (Karwat et al., 1999; Breitung and Royl, 2000; Bentaib et al., 2015; Lopez-Alonso et al., 2017), because deflagration or detonation might challenge the structural integrity of the containment. The concern about hydrogen risk and the demand for additional research on accident scenarios and on mitigation measures grew after the Fukushima accident (Liang et al, 2015; Gupta, 2015; Nishimura, et al, 2015). In particular, hydrogen stratification can substantially increase the risk, as this could lead to local pockets of mixtures with high concentration of this flammable gas (Choi et al., 2001). A special concern is thus the build-up and persistence of stratification of hydrogen in certain regions, which has to be addressed by dedicated experimental research and accurate analyses (Smith, 2009). Various experimental programmes (Allelein et al., 2007; Deri et al., 2010; OECD/NEA THAI Project, 2010; Allelein et al., 2012; Studer et al., 2012; Paladino et al., 2013; Kapulla et al., 2014) and code validation activities (Schwarz et al., 2011; Kelm et al., 2016a; Andreani et al., 2016a; Sarikurt and Hassan, 2017; Abe et al., 2018) have included in-depth investigations on stratification formation and break-up/erosion processes. This research included, among others, the investigation on the interaction of a gas plume or jet with a density interface in an open space, typical of the situation in the dome of the containment. However, since the multi-compartment geometry of many containment designs is quite complex, it is necessary to assess the capability of the codes to simulate the effect of various structures on the evolution of the distribution of gases in the presence of flow obstructions. In particular, the effect on mixing of an obstruction at short distance from the origin of the efflux is of general interest (e.g., Noutsopolos and Yannopoulos, 1989), but only a few investigations with light gases exist in open literature (e.g., Chan and Jones, 1997). A first series of experiments in PANDA addressing this issue have been conducted within the HYMERES project.

HYMERES (HYdrogen Mitigation Experiments for REactor Safety) is the acronym for an OECD/NEA project (2013-2016), which is supported by thirteen countries and centered around experiments performed in the PANDA and MISTRA facilities, located at PSI in Switzerland and CEA in France, respectively (Paladino et al., 2012). The project includes various series of experiments (with helium used as simulant for hydrogen), where the mixing of a stratified atmosphere is controlled by jets or energy sinks/sources (such as heaters, coolers, etc.). In PANDA, stratified conditions, with a helium-rich layer at the top of one steam-filled vessel, are either initially prescribed or built during the transient. One of these experiments in PANDA DP1_6_2) was chosen for the present benchmark, which is similar to the recent OECD/NEA PANDA Benchmark (Andreani et al., 2016a). It addresses again the stratification erosion induced by a vertical jet, but in this new test, with injection of steam instead of air, and with an obstruction above the pipe exit. The OECD /NEA PANDA benchmark indicated that even for simple conditions the successful application of CFD to containment flows is 
still hindered by the special feature of the typical problems, i.e. long transients in very large fluid domains. Under these conditions, the application of Best Practice Guidelines (Mahaffy et al., 2015) proves to be very demanding, and often nearly impossible with the available project times and computing resources. Benchmarking activities (especially based on blind predictions) are therefore very important to reveal the weaknesses of modelling strategies established on the base of previous experience when a new problem has to be tackled and sensitivity studies must be limited to a few runs.

In order to enhance the interpretation of the comparison of the calculated results with the experimental data, for the blind benchmark the participants were requested to submit two sets of results: one set should be obtained using a "Gmon model", and a second set produced by a "best estimate" model. For the "common model", a list of recommendations was given with respect to initial and boundary conditions (e.g., homogeneous initial vertical gas and wall temperatures, modelling of the injection pipe), as well as concerning model selection (no condensation, no radiative heat transfer, standard k- $\varepsilon$ turbulence model). For the "best estimate" model, each participant was free to choose the modelling approach that was considered to be the best suited to the physical problem investigated, also on the base of previous experience, and to use refined representation of initial and boundary conditions. Each participant was expected to submit only one set of results for each of the two models. The participants were encouraged to use Best Practice Guidelines (BPG) to provide the most trustworthy set of results, but only few participants could afford more than a mesh sensitivity study using two meshes.

Both sets of submissions, i.e. those using a "Common Model" (CM) and those using a "Best Estimate Model" (BEM) model produced a large variety of results, leaving open a number of questions. The open phase of the benchmark was then considered necessary to provide more information on the importance of some physical effects and the capability of the various modelling strategies adopted by the participants to address them.

The present paper reports the outcome of the benchmark. Results for the entire set of variables requested are collected and discussed in Andreani and Paladino (2018), which will be referred to as the Benchmark Report (2018). Here, only the main aspects of the benchmark are discussed. Since the results of the phase were already illustrated in Andreani et al. (2016b), the paper focuses on the open phase of the benchmark. 


\section{THE EXPERIMENT}

Only the essential information necessary for comparing the test results and the simulations is provided in this chapter. A complete presentation of the tests and of the main experimental results is included in a project report (Kapulla, et al., 2015a).

\subsection{Configuration and test conditions}

The PANDA facility (Paladino and Dreier, 2012) is a multi-compartment, large-scale thermalhydraulics test rig located at the Paul Scherrer Institute (PSI), Switzerland. For these series of tests only the two upper vessels were used (Fig. 1, where the large manholes at the top of the vessels are also visible), which are $8 \mathrm{~m}$ in height and $4 \mathrm{~m}$ in diameter. $\mathrm{In}$, the nominal conditions of the test are indicated. The PANDA vessels and the major internal penetrations/flanges are made of stainless steel. All external surfaces are insulated, and the heat losses have been experimentally determined over a broad range of temperatures. The experiment mainly addresses the evolution of the thermalhydraulic variables in the vessel (injection vessel, on the left in the figure) where the initial stratification was created and the steam was injected. In the injection vessel (Vessel 1), a steam jet originates from a circular pipe located on the axis of the vessel and with an exit located $2 \mathrm{~m}$ below the bottom of the helium-rich layer (which starts at $6 \mathrm{~m}$ ). The mixing is somewhat slowed down by a small circular plate $(20 \mathrm{~cm}$ in diameter) also centered on the axis and positioned $1 \mathrm{~m}$ above the jet exit. The vessels are kept at approximately constant pressure (1.3 bar) during the test by venting the fluid to the atmosphere through a nozzle at the top of Vessel 2.

Prior to the test, saturated steam was injected in the vessels, and the fluid and vessel walls (as well as the obstruction plate) were thus heated to the target temperature, which was set to avoid wall condensation during the transient. Stratified steam/helium conditions then have been created in the test vessel by injecting helium above $6000 \mathrm{~mm}$ (Fig. 1). Just before the beginning of the test, a helium-rich layer occupies the region above the elevation of $5000 \mathrm{~mm}$ (measured from the lowest point on the inside of the vessel), the molar fraction of helium increasing non-linearly to about 0.22 at $8000 \mathrm{~mm}$ and above into the manhole space. The region of Vessel 1 below this layer and Vessel 2 are filled with steam. The air concentrations were between 0.1 and $0.2 \%$. The measured helium molar (5) tion at time $\mathrm{t}=0$ as a function of elevation is also displayed in Fig. 1. All concentration measurements are subject to total combined uncertainties of $<1 \%$. The measured gas and wall temperatures immediately before the start of the transient were between 105 and $108{ }^{\circ} \mathrm{C}$ (nominal value: $108{ }^{\circ} \mathrm{C}$ ). These values ensure that condensation (if any) could not play a role during the transient. All temperature measurements are subject to an uncertainty of $\pm 0.7 \mathrm{~K}$. 
The test was started by injecting superheated steam, the flow rate and the temperature being $60 \mathrm{~g} / \mathrm{s}$ and $150{ }^{\circ} \mathrm{C}$, respectively. During the test, gas and wall temperatures at several locations were measured, and helium concentration was measured at 6 elevations above the injection. Moreover, PIV measurements in a zone of fluid initially immersed in the helium-rich layer (far above the plate) provide average velocities and turbulent quantities, and thus some information on the interaction between the jet and the stratified ambient (see below).

\subsection{Main results}

In the experiment, the histories of gas temperatures and helium concentrations above the injection describe the progression of the erosion process (Fig 2), $\mathrm{Ch}$ is characterized, at each elevation, by helium concentration drop and temperature increase as a result of the rise of the leading edge of the steam jet and associated mixing (D) intain) zone to that height. Since the test was rather fast (within $600 \mathrm{~s}$ the fluid in Vessel 1 was fully mixed), and the scanning rate of the mass spectrometer is necessarily low, the time history of the erosion at the various elevations (which will be used to get a representative measure of the global success of the simulations) was obtained from the temperature measurements (scanning rate $0.5 \mathrm{~Hz}$ ), using a shold of $120^{\circ} \mathrm{C}\left(\mathrm{T}_{\lim }\right.$ in Fig. 2). This procedure for defining the "erosion times" and comparing the measurements with code predictions at the various locations is clearly not accurate, especially at elevations (notably the highest at $8 \mathrm{~m}$ ) where (1) temperature has a slow and non-uniform increase. Another option would have been to use the times of the inflection points (maximum time derivatives of the temperature curves), but the results would differ from those obtained with the used procedure at most by $50 \mathrm{~s}$ (at the highest elevation). The experimental values will be shown together with the results of the simulations in the next section.

Mean velocity measurements were obtained by PIV in a Field of View (FOV) located between the elevations of 6300 and $7000 \mathrm{~mm}$. Measurements and discussion of statistical errors are included in the project report (Kapulla et al., 2015a). Since the averaging period was of $409 \mathrm{~s}$ (centred around $\mathrm{t}_{\mathrm{c}}=$ $326.6 \mathrm{~s}$ ), the use of this information for comparing the simulations with the data at specific times is questionable. The use of the velocity measurements for assessment purposes is reconsidered in the open phase of the benchmark, for which additional data for similar conditions over a larger region above the plate (between 5000 and $7000 \mathrm{~mm}$ ) are made available. Therefore, for the sake of the open benchmark, results for velocities will not be discussed.

\section{RESULTS OF THE SIMULATIONS FOR THE BLIND BENCHMARK}

It was mentioned above that the participants in the benchmark were requested to submit two sets of results, one using the "common model" (CM) and one with the "best estimate model" (BEM). The 
specifications for the CM (Andreani et al, 2016b) Duded geometry, treatment of heat transfer at the walls (heat losses, no radiation and no condensation), initial and boundary conditions, and prescribed use of the dard high-Reynolds number k- $\varepsilon$ turbulence model.

The participants were expected to deliver the times of the erosion progression, the time histories of helium concentrations and gas temperatures at selected locations, as well as axial and transversal profiles of gas temperature and velocity at various elevations, at selected times. Moreover, the organisations delivered an accompanying document with some information on physical and numerical models.

Not all organisations submitted both sets, and two organisations submitted results with two different codes. There were thus 13 contributions with the CM and 10 with the BEM. Table 1 summarises the submissions. In table 1, only the code used the total number of cells in the mesh, the main deviations of the CM from the specifications and the differences between CM and BEM are listed.

\subsection{Results with the Common Model (CM)}

Table 1 shows that various codes (mostly commercial CFD codes) have been used, with a broad PCtrum of meshes, from 20000 cells to more than 1 million. The CM simulations were mostly performed with models complying with the specifications, the only important deviations being those referring to initial and boundary conditions. The non-obvious effect of small differences in boundary and initial conditions was not anticipated by some users. This is a good example of the importance of the benchmarking activities for establishing CFD modelling strategies to be used for the analysis of new problems. Therefore, the comparison with data of the simulations using inaccurate values of steam injection temperature and initial helium concentration profiles is somewhat affected by this deviation in the test conditions. For these contributions, the discrepancies with the data and other simulations are easy to explain. However, also for the other contributions, a very broad variety of results has been obtained. Figure 3 shows the time history of the erosion progression and, as example of gas temperature time histories, the results for one elevation. It can be observed that the mixing time (600 $\mathrm{s}$ in the experiment) is well predicted in only three simulations (AERB, IBRAE2 and PSIF), whereas most submissions exhibit either a strong underprediction or overprediction. Considering the essential elements of the setups for the simulations (Table 1), but also the additional information provided, it is not obvious how to associate the success of the predictions to any specific difference between models. Since the physical models (and their implementation in the codes) must be very similar for all simulations, the differences in the prediction can be due to the mesh (refinement in certain regions and mesh topology), numerical methods, and other effects and modelling options not considered in the specifications (e.g. wall treatment). therefore surprising that models with largely 
different number of cells lead to comparable predictions, and, vice versa, the use of detailed models with similar number of cells result in very large differences. Although post-test analyses (see below) shed light on some of these apparently puzzling results, the observed spread suggests that the set-up of a CFD simulation for a new problem is not a trivial task, and requires a careful evaluation of the applicability of previous experience for different geometrical configurations and physical conditions.

Another interesting observation concerns the temperature rise: in all simulations (and at all elevations) this is sharper than in the experiment, with the asymptotic temperature being much higher than the measured $\rightarrow$ e. The temperature trend therefore reveals a systematic difficulty to predict the erosion process, which is not shown in most concentration histories (Fig. 4) where, because of the low data frequency, the calculated sudden drop seems to be always in agreement with the experimental results. The temperature distribution above the plate is, in general, reasonably well predicted in most simulations, where the horizontal profiles, in agreement with the experiment, show low peaks along the axis. Figure 5 shows, for example, the profile at some distance downstream the plate (Level G, $\mathrm{z}=5.63 \mathrm{~m}$ ). However, with the exception of one simulation, the profiles are systematically shifted towards higher values, indicating that some mixing and/or heat transfer mechanism (convective or radiative heat transfer) $\bigcirc$ ot well predicted.

Indeed, it seems that most simulations over predicted the gas temperatures already in the region below the plate. Figure 5 shows the temperature horizontal profile just below the plate (Level $\mathrm{H}, \mathrm{z}=4.97 \mathrm{~m}$ ) at $150 \mathrm{~s}$ : the temperature drop between the pipe outlet and the plate along the axis is underpredicted. This discrepancy, in principle, could be due to the fact that the codes predict perfectly symmetric flow, whereas in the experiment the jet was not perfectly centred due to flow non-symmetry at the pipe outlet, and thus the measured temperature does not represent the value (the maximum) at the jet impingement point. On the other hand, the deviation could reveal unexpected difficulties in predicting the broadening of the free jet. This question has been tackled in the open phase of the benchmark (Section 4).

\subsection{Results with the Best Estimate Model (BEM)}

The simulations with the CM provided two main results: 1) even using very similar models (apart from the mesh) very different results can be obtained, in spite of the use of meshing strategies that could be considered "qualified" on the basis of previous experience with various tests in PANDA; 2) large discrepancies between calculated and experimental results were obtained. With respect to this second result, the agreement was expected to improve with the use of the BEM, where the participants could make use of the best modelling approach according to their experience. This mostly resulted (Table 1) in the use of a different turbulence model, the consideration of radiation heat transfer, and 
initializing the simulations with the experimental gas and wall temperature distribution. It is here behaves as a participating medium (Abu-Romia and Tien, 1967; Howell, 1988), and therefore the radiation models consider not only heat transfer between solid surfaces, but also between gas and wall and within the gas.

Figure 6 shows the results for the erosion progression (calculated from the temperature rise) and the time history of the helium concentration at the highest elevation. Comparing results with those shown in Figs. 3 and 4 for the simulations with the CM, it can be noted that in a few cases the BEM results are better than the CM results (especially in the case with the largest discrepancy the use of different models produce a dramatic improvement, which was discussed in Andreani et al., 2016b), but in some cases the discrepancies are larger than using the CM. Three results are rather surprising: 1) the spread of results is still quite large; 2) the "best" results are obtained with a rather coarse mesh (163000 cells); 3) two simulations with identical code and very similar models (GRS and FZJ), which using the CM exhibited very different results, are now nearly coincident. This shows that the effect of the mesh possibly depends on the interaction with other modelling choices (turbulence model, numerical methods, boundary conditions, etc.).

It is also interesting to observe that the three simulations where radiation heat transfer was modelled result in a noticeable speed-up of the erosion process, due to the generation of additional convective motions in the upper part of the vessel, which enhance the mixing (Kelm et al. 2016b). For one simulation (CIEMAT), the faster mixing is uniquely due to radiative heat transfer, since the turbulence model was not changed in the BEM with respect to the CM. Considering radiative heat transfer helped in improving the prediction of the gas temperatures at some elevations but not at other ones. Moreover, the results for wall temperatures are somewhat contradictory, because the simulations using radiative heat transfer show qualitatively different results. This indicates that the simulation of radiation heat transfer (in combination with other models, first of all turbulence) requires additional investigations. The possible role played by radiation heat transfer in the process of gas mixing has, among others, also been recently suggested by Filippov et al. (2017).

\subsection{Conclusions and open questions originating from blind simulations}

h sets of submissions, i.e. those using a 9 mmon model" (CM) and those using a "best estimate" model (BEM), produced a large variety of results. Especially the large differences in the results with the $\mathrm{CM}$ were not expected because, in order to establish some means for comparing the results, a 
comprehensive set of specifications have been recommended. The open phase of the benchmark was then considered necessary to provide more information on effect of meshing strategies.

The comparison between predicted and experimental results, as well as between simulations, raised a few questions about the actual importance of considering radiative heat transfer, the relation between mesh topology and other modelling aspects (turbulence model, numerical methods, boundary conditions, etc.), and the effect of some test conditions that were not modelled (e.g. non-symmetry of the flow at the outlet of the pipe).

The most important difficulty in interpreting the results and finding the reason of the large deviations No the lack of information on the velocity field downstream of the obstruction. In fact, the interaction between the jet and the density stratification is expected to be controlled by the velocity distribution at the interface between the rising jet and that interface. Since the velocity field changes in time, due to the upwards movement of the leading edge of the jet, a meaningful comparison would require a time-dependent experimental information. Unfortunately, due to the short duration of the test, only long-term averages (on the time scale of the experiment) can be generated, and the comparison between the requested calculated instantaneous velocity profiles and these averaged values could not provide any hint on the fidelity of the simulation. One of the main difficulties to interpret the large spread of the erosion times is thus the lack of experimental information on the flow field, not only because of the problems discussed above but also because this is only available for a limited PIV area, which is at large distance from the obstruction. Therefore, an open benchmark using only the experimental information of test HP1_6_2 would lead to little progress in the understanding of the erosion process above an obstruction. The availability of velocity information under quasisteady state conditions collected in auxiliary tests (Section 4.1) without helium ("zero" tests), but with the same geometrical configuration, permitted, however, to obtain new information to be used in the evaluation of the calculations. In fact, although, in principle, one would need information on flow patterns and temperature field below and above the plate for the specific test, some progress in the analyses could be expected from the use of information on the extended region above the plate provided in these "zero" tests.

It was then agreed that a meaningful open benchmark could be run, but this should include the analyses of the data on flow obtained in auxiliary tests, and should be organized in a number of steps (see next chapter), to gradually build confidence in the models used. 


\section{THE OPEN BENCHMARK}

To help resolve the questions resulting from the blind phase, an open phase of the benchmark was conducted, where the extended data from the auxiliary "zero" tests (for similar conditions but without helium layer) were provided to the participants to permit a more basic validation of their models, by comparing the results for the flow downstream of the obstruction, which, in turn, are affected by the accuracy in simulating the free jet below the obstruction. A "cascade" of simulations was thus proposed, which aimed to separate the validation of the modelling approach for representing pure fluid dynamic phenomena from the application of the selected methodology to test HP1_6_2, where also heat/mass transfer effects play an equally (or even more) important role.

For each step, a summary of the contributions will be presented in tables, which include only the main information on the mesh and modelling options. The tables include the equivalent CPU time using one processor to provide a unified estimate of the computational overhead. A more complete list of aspects considered in the simulations and details on model selection is included in the Benchmark Report (2018).

\subsection{The "zero" tests}

The two configurations (and PIV windows) for the tests with obstruction are shown in Fig. 7. In the complementary, "zero", tests, the geometry was exactly the same as for Test HP1_6_2, the only difference being the initial and boundary conditions. Additionally, it is noted that for the "zero" tests the zone covered by PIV measurements spans a much larger zone, extending $2 \mathrm{~m}$ above the obstruction.

These tests were conducted injecting a constant steam flow rate in initially steam-filled vessels. In two tests (HP1_6_0 and HP1_7_0), pure steam was injected in steam-filled vessels, and the pressure was kept constant. The only difference was the flow rate.

These two tests, which were expected to deliver complementary information with respect to Test HP1_6_2, were used for the benchmark. A third test (HP1_8_0), which was performed with air and steam, and rising pressure was not used. For the two tests HP1_6_0 and HP1_7_0, the following nominal conditions apply:

- Pressure: 1.3 bar 
- Initial steam and wall temperature: $108^{\circ} \mathrm{C}$

- Injected steam temperature: $150{ }^{\circ} \mathrm{C}$

- Steam flow rate: $60 \mathrm{~g} / \mathrm{s}\left(\mathrm{HP} 1 \_6 \_0\right) ; 30 \mathrm{~g} / \mathrm{s}\left(\mathrm{HP} 1 \_7 \_0\right)$ Although test HP1_6_0 was the only with the same boundary conditions as 1 -6_2, the analysis of
second test was also included, because in test HP1_6_0 temperatures were not recorded.

The open phase of the benchmark thus consisted of a number of steps, aiming to separate the qualification of the fluid-dynamic models from the validation of the full models for test HP1_6_2, for which the success of the predictions also depend on the appropriate representation of heat and mass transfer processes. The open phase of the benchmark was thus composed of four sets of results:

Q rediction of vertical velocity and turbulent kinetic energy (TKE) distributions for Test HP1_6_0 using a "common model" (CM);

2) prediction of vertical velocity distributions and temperatures for test HP1_7_0 using a "bestestimate model" (BEM);

3) prediction of vertical velocity, turbulent kinetic energy and temperatures (although not measured) for test HP1_6_0 using the BEM;

4) post-test simulation of test HP1_6_2 with BEM, including the same mesh for the plate region used for Step 3

Additionally, also the analysis of SETH-2 test ST1_2_2 (Erkan et al., 2009; Paladino et al., 2013), with the same initial and boundary conditions as for test HP_6_2, but without obstruction, was included as Step 5, to verify the capability of the models to properly predict the erosion process in absence of the obstacle. This step was optional for participants.

\section{Step 1 (analysis with CM of test HP1_6_0, where temperatures were not measured)}

The first step of the open benchmark was the analysis of test HP1_6_0 (with the same flow rate, 60 $\mathrm{g} / \mathrm{s}$, as in test HP1_6_2), using the CM model, i.e., using a common set of recommendations regarding modelling selection and initial and boundary conditions:

- In order to isolate possible spurious sources of deviations in the final Step 4 (post-test calculation of Test HP1_6_2), also test HP1_6_0 had to be run using the full geometrical model, including the straight portion of the inlet pipe 
- Initial fluid and wall temperature: $107{ }^{\circ} \mathrm{C}$; Pressure and inlet flow rates: experimental curves. Flat pipe outlet velocity profile. Pipe exit turbulence intensity: $4 \%$.

- Fluid temperature at pipe outlet: $142{ }^{\circ} \mathrm{C}$ (precise value not critical, but important)

- Obstruction plate heat capacity included.

- Heat losses, condensation and radiative heat transfer neglected. Wall heat transfer to be considered: inner surface wall temperature to be prescribed (constant, at $107{ }^{\circ} \mathrm{C}$ )

- Standard high-Re k- $\varepsilon$ turbulence model with Standard wall functions

- Value of $\mathrm{y}+$ in the cells below the plate between $\mathrm{R}=3$ and $10 \mathrm{~cm}$ around 30.

- Recommended simulation time: $500 \mathrm{~s}$

The summary of contributions is listed in Table 2

It is noted that most organizations contributed results using a finer mesh than that used for the blind benchmark, two used the same mesh, and one used a coarser mesh. Some of these choices are justified in the Benchmark Report (2018). None of the submitted contributions was based on a comprehensive sensitivity study aiming to obtain mesh-insensitive results.

The first variables to be considered in the comparison between data and calculated results are horizontal and vertical profiles of vertical velocity, extracted from the velocity field. Figure 8 shows the experimental information (averaged over 205 s), along with the elevation of the selected horizontal profiles. Figure 8 also includes the experimental profiles of axial velocity, maximum of the vertical velocity and difference between the maxima in the left and right half-plane in the PIV window (this last being and indicator of the non-symmetry of the flow past the obstruction). $\mathrm{Y}_{1}$ and $\mathrm{Y}_{2}$ are the elevations where the vertical velocity changes sign and the maximum coincides with the axial value, respectively. The former is the height of the recirculation zone (bubble) downstream of the obstruction; the latter indicates the position where the flow recovers the structure of a full jet (with maximum in the centre).

Figure 9 shows the calculated results for the vertical profiles of the axial velocity and the maximum vertical velocity. The comparison of the axial profile below the plate (between 4000 and $5000 \mathrm{~mm}$ ), although no data are available for evaluating the quality of the predictions, is of some interest because it shows that very large differences (also qualitative) exist between the simulations of CIEMAT and BARC and the other ones. However, the differences are substantially reduced in the region downstream of the plate, with maximum values being in a band of $0.25 \mathrm{~m} / \mathrm{s}$, which indicates a 
relatively small effect of the predictions below the plate and that most differences above the plate originate from the representation of the flow obstruction region. In fact, simulations showing the same flow development below the plate (S/NRA/R, KAERI and FZJ), diverge above the plate. The simulation of FZJ produced the highest values of the velocity at the top of the measurement region. It is also interesting to observe that the velocities are still growing with distance at the top of the measurement region, whereas the experimental values reach the maximum at lower elevation (between 6000 and $6500 \mathrm{~mm}$ ). Finally, the module of the minimum velocity in the "bubble" above the plate is underpredicted in all simulations. The vertical distribution of the maximum velocity also shows a similar spread of results, with all calculations underpredicting the distance from the plate where the maximum attains its highest value. Two calculations also overpredict the highest values.

The comparison of the velocities is to some extent affected by the still slowly evolving flow field in some calculations, which is well recognized in the time history of the maximum velocity shown in the Benchmark Report (2018), which is not constant at the end of the simulation. In the experiment, however, the overlapping of the long-time average central profile using the entire data set with results using sub-sets indicates a practically steady-state condition. The reason for the evolving flow field is the slow fluid temperature increase, possibly due to the underprediction of heat transfer with the structures.

However, considering that some differences in the velocity distribution originate from the way the experimentally unknown temperature field is predicted, the agreement between predictions is reasonably good. The differences are larger for the Turbulent Kinetic Energy (TKE) in the recirculation zone (Fig. 10), whereas in the developed flow region the simulations converge to the experimental value. The large (also qualitative differences) in the distributions are thus confined to the recirculation region, and decay with distance from the obstruction.

An important feature of the transient that could not be investigated in test HP1_6_2, but could have an effect on the interaction of the jet with the density interface, was the symmetry of the flow structure and the flow evolution downstream of the plate. Asymmetric flow could either be produced by the impingement or directly originated at the outlet of the pipe. Whereas the flow below the obstruction cannot be observed also in the "zero" tests, its structure above the plate could be characterized, and it is reasonable to assume that it is representative of that produced in the reference test with helium. Therefore, an important aspect of the assessment of the models using the data from the zero tests is to verify whether the models correctly predict non-symmetries (if any) in the flow. For Step 1, since the recommendations included the modelling of the straight section of the pipe only, the verification only regards the production of non-symmetries in presence of axis-symmetric injection conditions.

Figure 11 shows the difference between the maxima in the two half-planes of the PIV window, which has a maximum in the zone close to the obstruction and vanishes within half a meter downstream of it. 
St simulations do not display any substantial asymmetry in the flow field past the plate. Only the contributions from AERB and BARC present a qualitatively important deviation from symmetry, the former in the recirculation zone, the latter a slowly increasing difference with distance from the plate. Figure 11 also shows the elevation where the flow recovers the jet structure (with maximum velocity in the centre). It can be observed that the predictions, although obtained with the same turbulence model, are in a large band. Considering the 2-D velocity maps provided by the participants (Benchmark Report, 2018), however, it is presumed that at least a part of the differences could be due to the different criterion used to determine the condition of "equality" between axial and maximum vertical velocity.

It has already been observed that large discrepancies exist in the recirculation zone between calculations and experiments, and between the calculated results. This is more evident from the time history of the minimum velocity (Fig. 12), which shows underprediction in all simulations. It is surprising, however, that, in spite of the large differences in the velocity profile, the height of the recirculation zone is well predicted in all calculations.

Finally, the horizontal profiles of velocity and TKE at the two elevations indicated in Fig. 10 are shown in Figs. 13 and 14, respectively. With respect to velocities, at the elevation of the middle of the recirculation zones, with the exception of a narrow region around the axis, the simulations are in agreement with each other, and also in agreement with the measurements. A large spread of results, however, is exhibited by the turbulent kinetic energies, as regards both maximum values and profiles.

Similar considerations also apply to the elevation at large distance from the obstruction, where the velocities are in good agreement (especially outside the jet core), but the calculated TKE shows a large variety of values and horizontal profile shapes.

These profiles indicate that even using the same turbulence model, the results can be quite different, depending on other modelling choices. Large difference between the results obtained with GOTHIC and those produced by CFD codes had to be expected because of the large differences in mesh detail (Table 2). The differences between CFD codes, however, are more difficult to justify, also because no correlation can be easily established between number of cells and main features of the calculated profiles. These results suggest that the mesh topology (and possibly details of the numerical model) could play an important role.

The general remark on the results of Step 1 is that even for the simplified conditions of HP1-6-0, important differences are observed, which are not related to turbulence modelling (the same standard $\mathrm{k}-\varepsilon$ for all) or radiation heat transfer (not considered), but probably originating mostly from the mesh (and topology) and, to a lesser extent, heat transfer and implemented boundary conditions. 


\subsection{Step 2 (analysis with BEM of test HP1_7_0, where temperatures were measured)}

Since temperatures were not recorded for Test HP1_6_0, and models constructed for best estimate simulations of the benchmark test should be validated also for their capability to properly predict fluid temperatures, the analysis of test HP1_7_0 is considered, although the steam mas flow rate was the half of that in test HP1_6_2 (30 g/s instead of $60 \mathrm{~g} / \mathrm{s})$.

In this test, the Reynolds number was thus 13500 instead of 27000 . Since the effects of the Re number, at least on free jet flow development, are significant up to Reynolds numbers around 10000, and the TKE decay downstream of the plate is similar in the two tests, HP1_7_0 could be used for developing the BEM modelling approach to be used for HP1_6_0 (and HP1_6_2). In particular, in the multi-step approach for the open benchmark, the comparison of the results with test HP1_7_0 was intended to provide the validation of the models with respect to representation of heat transfer.

Table 3 reports the main features of the simulations contributed to the benchmark. Unless otherwise noted (FZJ and VTT), the mesh used is the as that used for Step 1. Most users adopted the SST turbulence model, one the RNG model and some retained the standard turbulence model choice. With respect to Step 1, other important model modifications in some contributions were the consideration of radiation and the velocity profile at the pipe exit pipe (obtained by representing the straight section of the pipe or prescribed on the base of off-line calculations of the flow inside the entire pipe)

Figure 15 shows the experimental flow field and the vertical velocity profiles to be used for the comparison. It is noted that both the height of the recirculation bubble and the height at which the flow recovers the full-jet structure are smaller than for Test HP1_6_0.

Figure 16 shows two vertical profiles of vertical velocity: the distribution along the axis and the profile of the maximum velocity. The general agreement between the various simulations is similar to that observed for Step 1, and, in the region above the plate, better for the simulations that exhibit a close agreement below the plate (KAERI, S/NRA/R and FZJ). However, the flow evolution below the plate has a minor effect on the velocity field above the plate. These results suggest that actually the jet "loses the memory" above the plate. 

lerpredictions in the far-field velocity larger than for Step 1 are observed in the results of CIEMAT. Worse results were also obtained by VTT. Although the changes in modelling certainly play a prevailing role, and some mesh modifications with respect to Step 1 complicate the picture, this result possibly indicates that the disagreement between simulations does not depend on the jet Reynolds number. Moreover, the interpretation of the results is complicated by certain instabilities and large asymmetries in the velocity field which were exhibited by some simulations, but not observed in the experiment (Benchmark Report, 2018). Some of the calculated asymmetries appeared already at the pipe outlet, produced by bent in the lower part of the injection pipe (Figs. 1 and 7). However, PIV measurements immediately above the pipe exit performed in other HYMERES experiments for similar conditions (Paranjape et al., 2018) indicated that a well-developed turbulent pipe profile existed at the pipe exit. Although at the higher velocity of Test HP1_6_2 the effect of the bent could in principle be larger, the considerations above related to test HP1_7_0 suggested that one of the open issues of the blind benchmark, namely the effect of possible non-symmetries at the pipe exit the flow development above the obstruction, could be considered of minor importance for the evaluation of the results of the benchmark test. Detailed discussions on these and other aspects of the flow are discussed in the Benchmark Report (2018).

Concerning the fluid temperatures, the time histories are presented and discussed in the Benchmark Report (2018). Here, we just mention that the two simulations including radiation heat transfer (FZJ and BARC) correctly predict the short time required for the fluid to reach equilibrium values with the structures, whereas the other predictions show still increasing temperatures at 1000 s. It can be observed that these two simulations exhibit the most accurate predictions for the axial temperature profile (Fig. 17). The largest deviations occur at the position close to the plate, due to the difficulty to correctly predict the details of the flow within the recirculation zone nchmark Report, 2018).

Concerning the radial profile at Level D (only this one was required in the benchmark specifications), both simulations show a too narrow profile (but this is true for all simulations) and underprediction of the temperatures at some distance from the axis (Fig. 17). Although the results of BARC are probably somewhat biased by a large (and difficult to explain) temperature drop below the plate and the radial distribution at $1000 \mathrm{~s}$ calculated by FZJ is affected (Benchmark Report, 2018) by a plume oscillation (still of small amplitude at level D), it can be concluded that the two simulations agree with each other with respect to the effect radiation heat transfer can have. Globally, these simulations can also be regarded as the most successful, since the contribution of VTT (which does not model radiation) also shows good agreement above the plate, but these good results are due to the lower injection temperature (it can be noted in the axial temperature profile, Fig. 17). 


\subsection{Step 3 (analysis with BEM of Test HP1_6_0)}

Step 3 was intended, in the multi-step approach of the open benchmark, to provide the participants the opportunity to validate the model selection made for test HP1_7_0 for the same flow as used in Test HP1_6_2, and to refine the mesh, if needed. In Step 3, additional horizontal profiles of velocity and TKE at low elevations were requested.

The overview of contributions for Step 3 is presented in Table 4.

Figure 18 shows the axial and maximum velocity vertical profiles. For convenience, the results obtained for Step 1 are also displayed using the same scales.

Two participants (BARC and CIEMAT) used the same mesh as for Step 1, whereas the other four users used slightly finer meshes, adopting modifications that were suggested by the simulation of Step 2. Four participants used the same model settings as for Step 2, but CIEMAT and VTT used different turbulence model (or corrections).

Figure 18 shows that differences in injection velocity for five participants (not considering the contribution of CIEMAT, which for Step 1 used a too low value) are now more pronounced than for Step 1, due to the different assumptions for steam injection temperature (prescribed or calculated) and, possibly, details of the mesh used for the pipe. The development of the flow immediately above the pipe is also affected by the use of a velocity profile at the pipe exit, so that the profiles below the plate diverge more than for Step 1.

In the region above the plate, results of BARC, CIEMAT, FZJ, and VTT are very similar to those obtained using the common model, and the results of S/NRA/R only show a noticeable difference for the maximum velocity. These results possibly indicate that the selection of the turbulence model, for these conditions, a small impact on the prediction of the global features of the flow. The largest differences between Step 1 and Step 3 regard the simulations of PSI and CIEMAT, where, in both cases, the pipe exit conditions were strongly modified with respect to the calculations with the common model. The calculations of PSI with the "CFD like" GOTHIC code were "worse" than with the common model, but, due to the more physical representation of the pipe exit conditions (pipe exit velocity and temperature profiles are considered), are the best suitable to be compared with CFD codes in this benchmark.

The axial profiles of TKE (Benchmark Report, 2018) also show that the selection of the turbulence model (different from the standard k- $\varepsilon$ model in all calculations but that of PSI) has a strong influence in the region immediately above the plate, but its effect is substantially reduced already at relatively short distance from the top of the recirculation zone. 
This consideration is especially true for the calculations of BARC, where the results with both developed to handle this kind of flows. The results of CIEMAT display the largest change, but for this simulation are not clear whether the large difference in mesh and inlet velocity were the most important effects. Finally, the radial profiles show that also the predicted axial values of S/NRA/R (for which no axial profile was provided) are less sensitive to the turbulence model selection than expected.

Obviously, these considerations cannot lead to the conclusion that the selection of turbulence model is immaterial for the representation of the flow past the obstruction and for the erosion process in transient test HP1_6_2, since an apparently small difference in the velocity and TKE distribution can have a large influence on the turbulent diffusivity distribution.

Indeed, the details of the results for Step 3 are also interesting in view of their prospective importance for the prediction of the benchmark test. Both velocity and TKE horizontal profiles show a large spread between the various simulations, and this suggests that the differences in the flow predictions could results in large differences in the erosion rate.

Finally, some considerations can be made on the predictions of fluid temperatures, although no experimental values have been recorded. It is observed that large differences exist in the free jet temperature decay (Fig. 19), although the steam injection temperature is the same, apart from the calculation of VTT. Moreover, the radial profiles, similarly to the results for Step 2, show that radiation heat transfer has a strong effect on temperature spatial evolution. It is also interesting to mention that the time to reach a quasi-steady-state condition is very different in the various predictions (Benchmark Report, 2018) and, for some simulations, do not appear consistent with the results (also experimental) obtained for test HP1_7_0 (Step 2). In consideration of similar differences between the various simulations observed for test HP1_7_0 (where experimental values were available), it can tentatively be concluded that the modeling of radiation also permits a better representation of the time development of the temperature field.

\section{$4.5 \sqrt{5}$ (analysis with BEM of test ST1_2_2, without plate)}

Before the post-test results for the benchmark test are discussed, the analyses of test ST1_2_2 are briefly illustrated. The analyses of this test (conducted with the same configuration used for HP1_6_2, with the only difference that no obstruction was present), were expected to offer the opportunity to 
PIV measurements at short distance from the pipe exit.

The configuration of test ST1_2_2 (Erkan et al., 2009; Paladino et al., 2013) is shown in Fig. 20, where the initial distribution of helium in the injection vessel is compared with that present at the start of test HP1_6_2. Figure 20 also shows the comparison of the other important boundary conditions, i.e. steam flow rate and temperature. The maximum helium concentration was slightly higher than in the benchmark test, whereas the steam injection temperature was lower. As for the steam mass flow rate, differences can be regarded as negligible.

It will be seen below that these conditions and the absence of the obstruction resulted in an about $30 \%$ faster stratification break-up.

Table 5 presents an overview of the four calculations contributed to this part of the benchm Figure 21 shows the progression of the erosion, based on the gas temperature threshold of $115^{\circ} \mathrm{C}$. This limit is lower than for HP1_6_2 because the steam injection temperature was lower (Fig. 20).

The simulations of the three organizations that submitted results also for the other steps (AERB, FZJ and PSI) accurately predict the erosion process, with some differences appearing only towards the end of the transient. The good predictions of these three contributions are also confirmed by the time histories of the helium gas concentration (Fig. 22).

The only notable discrepancy in the three simulations are the delayed helium concentration drop calculated by FZJ at level D $(\mathrm{z}=6.3 \mathrm{~m})$, which, however, may be due to a graphical representation effect, because (see Benchmark Report, 2018) the frequency of the required data (every 5 s) does not permit to display the calculated drop and recovery of the helium concentration during the time interval between two measurements.

Figure 23 shows the vertical velocity axial distribution and the horizontal profile at $\mathrm{z}=5003 \mathrm{~mm}$. It is observed that the calculation with GOTHIC (PSI) accurately predicts the maximum velocity at short distance from the pipe exit, whereas the other two simulations strongly overpredict it. The width of the profile instead is better calculated by the simulations of AERB and FZJ. It could be inferred that the velocity decay further up has been properly calculated in all simulations, because otherwise it would not be possible to capture the erosion rate. The analysis of the results of Step 4, however, will lead to different conclusions (see Section 4.6).

As regards the discrepancies at short distance from the pipe exit, although it can be presumed that they can partly be due to the slightly off-center position of the jet (in the third dimension), the results 
of AERB and FZJ suggest that either the pipe exit conditions or the near-field jet spreading is not properly calculated in their simulations.

This conclusion is supported by the observation of the axial profiles of gas temperature (Fig. 24) at two different times.

In both simulations of AERB and FZJ, the central temperature remains at the value of the injected steam up to above $5 \mathrm{~m}$, whereas the experimental value at that elevation has already dropped by more than $5 \mathrm{~K}$. In the calculation by PSI, however, the gas temperature drops smoothly to the lower measured value. The analyses presented in the Benchmark Report (2018) show that this result is due to the representation of both velocity and temperature profile at the pipe exit, due to the flow development inside the pipe and heat transfer with the pipe walls. In fact, if the velocity and temperature profiles are assumed flat, both axial velocity and temperature do not start decreasing below $5 \mathrm{~m}$.

It can be concluded that in the FZJ and AERB calculations either the entrainment in the free jet is too little, which produces a too small jet broadening, or the pipe exit conditions were not properly accounted for. For FZJ, the good agreement with the measurements for test HP1_7_0 (Step 2, half steam flow rate) with respect to both temperature (Fig. 17) and velocity profiles (Benchmark Report, 2018), suggests that the predictions are affected by the Reynolds number. The comparison between simulations for Test ST1_2_2 and HP1_6_2 (shown in the Benchmark Report, 2018) also indicates that the jet spreading below the plate depends on the evolution of the eddy diffusivity.

The elevation where the jet is fully developed (central temperature starts decreasing) is thus predicted at about $5000 \mathrm{~mm}$ and $5300 \mathrm{~mm}$ in the FZJ and AERB calculations, respectively. The underprediction of the jet spreading in the FZJ and AERB simulations can be recognized from the too narrow temperature profile, which is also present in the PSI calculation, although to a lesser extent.

This result suggests that also for Step 4 (to be discussed below) the simulations of FZJ and AERB should be affected by inaccuracies in the simulation of flow field and temperatures below the plate.

The axial temperature profiles show that only the calculation (FZJ) considering radiation heat transfer captures the correct level of temperature at large distance during the erosion process (time $<500 \mathrm{~s}$ ), although the temperatures drop between the elevation where the jet is fully developed and the bottom of the helium layer is overpredicted. In general, also the horizontal temperature profiles (Fig. 25) show a better success of the FZJ simulation, although not all results seem to offer a consistent picture (Fig. 26).

From the profiles in the mixed region below the tip of the jet (Benchmark Report, 2018) one can conclude that the modelling of radiation heat transfer was necessary to correctly calculate the fluid 
temperatures. Also at higher levels (Fig. 25 and 26), where the upwards propagation of the jet produced the temperature rise and helium concentration drop, the temperatures before the mixing (Level D) or during or immediately afterwards (Level C) are much better predicted by FZJ. At times after the mixing has occurred (250 s at level D and especially at $500 \mathrm{~s}$ at level C), however, the calculation of FZJ shows notable discrepancies, possibly due to the (small) asymmetry in the flow and temperature field appearing shortly before the dissolution of the helium layer is completed (Benchmark Report, 2018).

Some additional information can be obtained on the gas temperature time histories at the required positions (not shown). First of all, the three simulations of AERB, FZJ and PSI correctly predict the temperatures in vicinity to the pipe exit, which shows that the correct boundary conditions were applied and no numerical diffusion corrupted the simulations from the injection elevation. The best predictions were obtained by FZJ using radiation, although the results of PSI using a coarse mesh and a standard correlation for convective heat transfer are nearly equally successful.

The results for off-axis positions above the initial density interface are somewhat contradictory, with generally better results obtained by PSI, with the effect of radiation being to excessively reduce the temperatures during the entire transient and also after the full dissolution of the helium layer. The discrepancy is especially large at some positions during the erosion process. On the contrary, at lower positions, the calculation with radiation permits to reproduce the correct temperature, whereas the calculation with GOTHIC predicts a strong superheat, the largest differences occurring during the transient compression and erosion of the helium layer, before mixing. Although it cannot be excluded that convective heat transfer could also play a major role in the different predictions (since both turbulence model and meshes were different), it can be presumed that the largest contribution to the divergent behaviour could come from the modelling of radiation in the simulation of FZJ.

Since all positions where the discrepancies are very large are all in regions of slow velocities and even in nearly stagnant zones, these result suggest that the strongest effect of radiation could be observed in the zones where the temperature increase due to compression of the fluid cannot be contrasted by the weak convective heat transfer but is kept low by the effect of radiation heat transfer. Moreover, the results indicate that the effect of radiation heat transfer may show up more distinctly during transients rather under quasi-steady state conditions (such as those established in Test ST1_2_2 after the dissolution of the stratification), which would be justified by the different time scales of the convective heat transfer (which is bound to the finite propagation velocity through the fluid) and radiation heat transfer (which instead has practically an immediate effect). 


\subsection{Step 4 (post-test analysis of benchmark test HP1_6_2)}

The last step in the analysis is the post-test simulation of the benchmark test HP1_6_2. In accordance with the rationale for the multi-step approach, mesh around the plate and modelling would be expected to be the same as for Step 3 (HP1_6_0), with some corrections to the mesh in the main domain due to the knowledge gained from the analysis of Test ST1_2_2, featuring helium layer erosion without obstruction. Table 6, where the overview of the contributions is presented, shows that only FZJ and PSI ran all steps and followed this path in building the model used for the final calculation. Also BARC and S/NRA/R submitted results with same mesh and model setting, but opted not to perform the additional Step 5. It is also noted that one organization used a coarser mesh than for Step 3: CIEMAT model reverted to the coarse mesh model used for the blind benchmark.

Also with respect to the turbulence model, in some contributions Step 4 has not been analyzed with the same selection used for the other steps. As regards radiation heat transfer, it is noted that one contribution (VTT) used this model only for Step 4: therefore a preliminary validation using the data of HP1_7_0 an ST1_2_2 (Steps 2 and 5) was not performed.

Figure 27 shows the erosion progression determined using the times at which for each location the gas temperature rises to $120{ }^{\circ} \mathrm{C},{ }^{1}$ whereas Fig. 28 shows the helium concentration time history at level B $(\mathrm{z}=7.48 \mathrm{~m})$. In these figures, the results obtained in the open phase are compared with those submitted for the blind benchmark, using the best estimate model. It is noted that VTT did not contribute best estimate results for the blind benchmark, and thus no comparison is possible for this organization.

It is observed (Fig. 28) that several organizations that participated in the blind benchmark could obtain better results (especially BARC, S/NRA/R and GRS, with this third organization, however, supplying the final results with a model that was not verified in Steps 1 to 5). AERB, CIEMAT and Q , instead, practically obtained the same results as for the blind simulations. This outcome of the multi-step validation of the model for CIEMAT is obvious, because the final step was run with the same mesh and model settings as for the blind exercise. For AERB (Benchmark Report, 2018), the results (using a much finer mesh) have been shown to depend on the mesh around the plate and therefore on an accurate prediction of velocity field near the plate (comparing the results of sensitivity studies for Step 3), overall grid size in the stratification region (Step 5), and numerical scheme. On the other hand, the Realizable k- $\varepsilon$ was preferred to the standard and the RNG model only for the better convergence obtained with this turbulence model. For FZJ, the results could seem somewhat surprising, because for all other steps the model delivered rather accurate predictions, at least at some

\footnotetext{
${ }^{1}$ See Section 2.2. Results up to level B $(7500 \mathrm{~mm})$ are confirmed by the inspection of the temperature predictions. For level A (8000 mm), the "erosion time" is somewhat underestimated.
} 
distance from the pipe exit. It has to be considered, however, that the results of the blind benchmark have not been revised systematically applying the outcome of the other steps, since they used a Best Dimate Model constructed on the base of a comprehensive validation experience. Numerical effects were also investigated, but showed no considerable importance.

A possible contribution to the discrepancy can be due to the underprediction of the jet broadening below the plate, as suggested by the results of Step 5, and/or the slightly larger maximum velocity and TKE at high elevations (observed in Step 3). The difference in the results can be explained neither by the modelling of radiation alone (also considered by BARC, CIEMAT, GRS, and VTT), nor by mesh or turbulence model or injection conditions. It is likely that a combination of all these elements finally produced the large differences observed in the final results of FZJ for the transient test. Especially interesting is the comparison between the calculations of FZJ and BARC, because for Step 3 the results of both simulations were close to each other, the only important differences being the TKE magnitude at some distance from the plate, and the elevation of the full jet recovery.

This suggests that the results for the erosion progression are strongly affected by the turbulence model, and wall layer treatment of the jet impingement zone (including the best choice for the size of the cell adjacent to the plate). Some additional elements to partly explain similarities and differences between results will be provided by the analyses of the flow variables (see below), but only further analyses and sensitivity studies by the individual organizations could bring some light on the role played by mesh and modelling choice.

As for other simulations, the concentrations time histories confirm that FZJ and CIEMAT (and at late times also VTT) overpredict the erosion rate, whereas the others either capture or slightly underpredict the mixing rate. It is also observed that in the simulation of VTT, the erosion is initially slower (Level F, at $\mathrm{z}=6 \mathrm{~m}$ ), probably due to the delay in the reattachment of the flow above the plate (see below), which was observed for Step 2, but not for Step 3 (Benchmark Report, 2018).

As for the effect of modelling radiation (which, in the case of BARC lead to very accurate results, but, for FZJ resulted in too fast mixing), Fig. 29 shows the comparison of the results obtained by GRS (Schramm et al., 2017). For this simulation, the accelerating effect of radiation is very large. In other investigations (e.g. Kelm et al., 2016b) for similar conditions, radiation was shown to promote mixing due to its influence on temperature (and density) fields, although its effect was not as substantial as in the simulations shown here. Additional studies on the effects of radiation are presented in the Benchmark Report (2018). 
The apparently excellent global results obtained with GOTHIC and coarse meshes are probably affected by compensation of errors, as indicated by the comparison for the velocity.

Due to the large averaging time of the available velocity measurements, the comparison between the calculated axial and horizontal velocity profiles (Fig. 30) has to be taken with some caution, as shown by the large fluctuations in the measurements over a $10 \mathrm{~s}$ period around the central time ( $300 \mathrm{~s})$ of the averaging period. Nevertheless, it can be recognized that the velocity in the PIV window calculated by PSI is far too low, which suggests that the good predictions for the erosion result from the compensation of errors between the lower velocities in the far field above the plate and numerical diffusion. Since the results for Step 5 in the near field (without plate) were excellent and the results for Step 3 (without helium layer) were reasonably good, it can be concluded that the complexity of the transient test HP1_6_2 lead to overprediction of the velocity decay between the plate and the density (1) rface. It can also be observed that the same order (from faster to slower) in the erosion timing displayed by concentration and temperature traces can be found in the magnitude of the velocity: the simulation of FZJ, which predicts fast erosion, also predicts the largest velocity in the PIV window. Vice versa, the simulation of AERB, which underpredicts the velocity, also slightly underpredicts the erosion rate.

A special attention deserves the simulation of VTT, which used a very detailed mesh and calculated (as result from the detailed modeling of the injection pipe) an asymmetric velocity and temperature profile at the pipe exit. The evolution of the calculated flow field (Benchmark Report, 2018) shows for about $200 \mathrm{~s}$ a splitting of the velocity field above the plate, and only at about $220 \mathrm{~s}$ the plume/jet structure is recovered. Since for the same flow rate (Step 3) this delay was not observed (but it was observed in test HP1_7_0, Step 2), the results suggest that the density interface produced the same effect as a lower mass flow rate. No conclusive explanation could be found so far for the results concerning the recirculation zone above the plate. On the other hand, the simulation of BARC (taken as example, but similar behavior is displayed by FZJ and CIEMAT) also show an Den" flow structure at early times, but already after $100 \mathrm{~s}$ the full jet/plume is recovered. This behavior does not depend on the steam injection temperature (constant at $150{ }^{\circ} \mathrm{C}$ in the BARC calculation).

The observation related to the VTT simulation is particularly important, because it suggests that the set-up of a model based on the good results for simplified conditions can prove not to be equally successful when applied to the more complex situation of a test featuring transient behavior and strong density differences.

Some important observations can be made on the axial and horizontal temperature distributions (Figs. 31 and 32), Generally, the most accurate axial temperature distributions are obtained by the models including radiation heat transfer, but also the simulation with GOTHIC, which accurately accounts for the temperature drop between the pipe exit and the plate, displays a quite remarkable agreement. The 
initially too large temperature difference between the elevations below and above the plate calculated by VTT is related to the delay in the re-attachment of the flow (see above).

Due to the complexity of the phenomena, it is not possible to draw any conclusions, but it is simply observed that no obvious correlation seem to exist between accuracy in the calculation of the temperatures and mixing rate. The horizontal temperature profile immediately below the plate (Level H) shows that all simulations predict a too narrow peak, with rather flat profile at some distance from the axis. At this elevation, the most distinct difference between some simulations including radiation (CIEMAT, FZJ and VTT) and those not representing this heat transfer mode is the larger temperature drop between the centre of the jet/plume and the periphery of the flow. This, however, is not observed in the calculation of BARC.

For the region above the plate, the results are obviously affected by the different progression of the stratification erosion. It can be observed, however, that at intermediate elevations (Levels G and D) most of the simulations including radiation predict better the temperature profiles, especially the temperatures near the wall.

The results obtained with the models with radiation at the bottom of the dome (Level C) seem to be less accurate than those neglecting this heat transfer mode. In fact, whereas at $250 \mathrm{~s}$ the top of the jet has already reached that elevation in the simulations of CIEMAT, FXJ and BARC, and therefore the temperature profiles are not comparable, at $500 \mathrm{~s}$ in all simulations the mixing has been completed at level $\mathrm{C}$, and one can observe large discrepancies with data in the simulations with radiation (again, excluding BARC), of the same magnitude, though opposite in sign, as the other simulations.

The simulation of FZJ at $150 \mathrm{~s}$, moreover, is affected by asymmetry, similar to that observed for test ST1_2_2. In this case, it is easy to explain this profile with the bending and fluctuation of the plume, as shown by the temperature field at various times (Benchmark Report, 2018). It is interesting to note that the presence of a density interface acts on the plume as a lower flow rate. In fact, large-scale oscillations were predicted for HP1_7_0 (30 g/s), but not for HP1_6_0 (60 g/s).

The comparisons of the gas temperature time histories at various positions (Benchmark Report, 2018) permitted a better evaluation of the role played by radiation. Especially interesting is the consideration of the temperature level reached during the transient, the temperature after the stratification moved above a specific location, and the temperature rise time at the various locations.

An example of temperatures at higher locations is shown in Fig. 33. At the position along the axis (Fig. 33 left), the calculations can be divided in three groups: 1) the simulations with radiation are the most accurate with respect to the final values, but underpredict the times of the rise (erosion), with the exception of BARC; 2) the CFD simulations without radiation overpredict the temperature; 3) the 
results of PSI with GOTHIC are in between. Concerning the slopes of the temperature increase, the

Finally, the temperatures at two positions off-axis below the initial bottom of the helium layer are considered (Fig. 34). Close to the centre ( $325 \mathrm{~mm}$ form the axis), at the elevation of the recirculation bubble above the plate $\left(\mathrm{GH} \_19, \mathrm{z}=5.3 \mathrm{~m}\right)$ the results are in a band of $15 \mathrm{~K}$, with the PSI results being the most accurate. Further off-axis (about $900 \mathrm{~mm}$ form the centre), and at the level of the injection, therefore at a position weakly affected by convective motions, the CFD simulations with radiation show substantially better results than the other calculations. At this position (K_17, $z=4 \mathrm{~m}$ ), and this was the case also for test ST1-2-2 (Benchmark Report, 2018), the role played by radiation in nearly stagnant region appear more clearly. It can also be shown that in these regions, where the heat-up is initially mainly caused by compression, radiation is the only effective heat transfer process, since it does not require the development of the boundary layer, which affects instead convective heat transfer (Filippov et al., 2017)

It must also be remarked that the various predictions including radiation heat transfer produced different results, as expected from the use of different models and methods to calculate the steam absorptivity. The differences, also connected to the different time progression of the erosion, are large during the mixing process, and vanish at many locations after the helium layer has been dissolved.

\section{CONCLUSIONS}

The experiment in the PANDA facility chosen for the present benchmark, test HP1_6_2, addresses the stratification erosion induced by a vertical steam jet, which originates from the exit of a circular pipe located below the bottom of the helium-rich layer. The mixing is somewhat slowed down by a small, horizontal, circular plate above the jet source. The benchmark consisted of two phases: blind 
and open. The results of the blind benchmark exhibited a large spread of results, some showing very large discrepancies with the measured data, which was not expected, especially for the part addressing the use of a "common" model. The results of the blind simulations made evident that further investigations and validation studies were necessary to separate different sources of errors and avoid their mutual elimination (compensating errors) in a complex model.

It was recognized that the most important difficulty in interpreting the results and finding the reason of the large deviations was the lack of information on the velocity field downstream of the obstruction, since only long-time averaged velocities were available in the region of the initial density interface, above $6 \mathrm{~m}$. It was therefore agreed that valuable information on the flow produced by the interaction of the free jet with the obstruction could be obtained from auxiliary tests without helium ("zero" tests), but with the same geometrical configuration and featuring an extended region above the plate where velocity measurements were available.

Therefore, the open benchmark included the analyses of the data on the flow structure above the plate obtained in these auxiliary tests: a "cascade" of simulations was thus proposed, which aimed to separate the validation of the modelling approach for representing pure fluid dynamic phenomena (using the data of the "zero" tests HP1_6_0 and HP1_7_0) from the application of the selected mesh and models to the more complex test HP1_6_2, for which the success of the predictions also depend on the appropriate representation of heat and mass transfer processes

The open phase of the benchmark thus consisted of steps. Additionally, also the analysis of SETH-2 test ST1_2_2, with the same initial and boundary conditions as for test HP1_6_2, but without obstruction, was included as Step 5, to verify the capability of the models to properly predict the erosion process in absence of the obstacle, and therefore test their basic capabilities to represent the global features of the transient in the entire flow domain. Finally, also the velocity measurements at the pipe exit in two other test series were used in some comparisons to verify the appropriate representation of the injection conditions.

Since each participant was requested to submit one set (best estimate) of final results for each step and only the main sensitivity studies of some organisations were contributed to the final report, only general conclusion will be discussed, leaving the detailed answers to the questions to future publications of the individual organisations.

The simulations with a common model for the quasi-steady state conditions of test HP1_6_0 without helium (step 1) were expected to provide the opportunity to investigate the effect of mesh on the simulation of the interaction of the jet with the plate. The final results submitted exhibited notable differences, especially in relation to transversal distributions and flow development downstream of the 
plate. Although the simulations could be affected by numerics and the spurious effects of the calculated slow change of the thermal field, the analysis indicate that meshing strategies could not converge to produce similar results and setting an adequate mesh for representing the flow modification due to an obstacle poses a real challenge. It can be inferred that the variety of results later obtained for the full test are strongly affected by the meshes used in this region. This is somehow confirmed by the observations that the few simulations of test ST1_2_2 (Step 5) without obstacle were all reasonably successful, although performed with largely different meshes and model selections.

As regards the interaction of mesh and model selection (which produced in the blind benchmark astonishingly different results using a turbulence model but not a different one), no new information could be generated within this benchmark, due to the lack of systematic analysis and the use of similar turbulence models (all variants of $\mathrm{k}-\varepsilon$ and $\mathrm{k}-\omega$ models). However, comparing results for test HP1_6_0 with the "common" model and the "best-estimate" model (Steps 1 and 3, respectively), it was observed that the selection of models had a smaller effect than expected, and was seemingly less important than the mesh.

Thermal radiation heat transfer was confirmed to have an accelerating effect on the progression of the erosion process, independently of the specific model used, and a substantial part of the information obtained from the temperatures indicates that this heat transfer mode should be modelled to get the correct spatial and temporal evolution of the thermal field. However, since various simulations with radiation were only performed for the final step, some results appear to be contradictory, and inaccuracies (possibly compensated or enhanced by radiation) are certainly associated with the use of meshes not optimised for the flow investigated (see above) and with the possible interaction with other models and effects, no firm conclusion could be reached on the necessity and approach to model radiation heat transfer. This issue will certainly require further investigations, both experimental and numerical.

\section{definite conclusion could be reached with respect to best choice for turbulence model, as the} meshing appeared to be a more important issue for the conditions investigated in this benchmark.

The velocity and temperature distributions in the jet at the pipe exit have some effect on flow and thermal variables, but this is mostly confined to the region below the obstruction. Finally, the effect of numerical methods has been reported from some participants and for some contributions it can be suspected to be responsible for some anomalous results and the submission of the final results with meshes not optimised. However, the absence of systematic studies in most contributions show how difficult is to implement in the analyses of transients requiring hundreds hours of CPU a rigorous approach to guarantee mesh and time step independence. 
The main conclusion of the open benchmark is that the step-by-step validation permitted some gress with respect to some of the items identified above. However, large discrepancies with data in the final analyses of the test are observed, which cannot be easily attributed to specific model deficiencies. The uncertainty is partly due to the difficulty to perform exhaustive analyses for each step including all effects, partly to the physical model limitations (e.g. use of RANS models for turbulence), and partly to specific features of the reference test that cannot be tackled in simulations of simplified conditions. On the one hand, even for the simpler fluid conditions of the tests without density interface, mesh and models could not be fully assessed. On the other hand, it is clear that the complexity of the physical conditions prevailing in the selected test, where the modification of the flow produced by the obstacle interacts with the stratification erosion process, rendered the splitting of the problem in hydrodynamic and heat/mass transfer components of lesser use than anticipated.

A few general considerations should be added in relation to the blind benchmark and the large, unexpected, differences in the results. The spread of the results was similar, for instance, to the recent OECD/NEA-PSI CFD benchmark without obstruction, although specifications for the "common" model were given, which covered various aspects of the simulation (geometry, turbulence modelling, initial, and boundary conditions, some fluid and flow properties, etc.). Since other aspects of the simulation were not considered in the specifications (e.g. wall treatment), and therefore some of the differences could be due to specific code inputs as well as to the numerical methods used, and the level of validation (including mesh convergence studies and application of BPG) was different for the various contributions, it is difficult to draw any conclusions from the comparison of the requested results. However, it can be observed that, similarly to previous exercises for similar flows and configurations (but without obstruction), the present results suggest that whenever a new problem is tackled, established modelling strategies must be evaluated again. The outcome of the exercise reinforced the awareness of the spread of results that can be obtained if the adequacy of the mesh is solely evaluated on the base of previous experience and limited mesh refinement studies. In this respect, the fact that some of the best results were obtained with rather coarse meshes should not be used as argument in favour of this approach for applications, without appropriate validation. Furthermore, even the use of very detailed meshes, resulting from systematic studies (going close to the full application of the BPG) does not lead necessarily to similar results, which are possibly affected by the topology of the mesh, local refinement, details of the numerics, and other effects.

These considerations lead to a few key-findings of this comparison. First of all grid independence must be proven for each physical and geometrical model as well as set of boundary conditions and cannot be simply assumed that this can be concluded from a similar case. Small changes in the flow/setup, here the implementation of a small flow obstruction, may challenge the model validity range. This suggests that continuous validation and a backward assessment of previous results (e.g. those obtained for the OECD/NEA benchmark or other tests addressing stratification break-up) is 
necessary. The results furthermore highlight that the effect of user-defined numerical or physical assumptions is in the order of those of model differences.

In summary, the results highlight, for the sake of more precise conclusions, the need for proper grid convergence studies, beyond the prescriptions of the BPG (not addressing mesh topology), which possibly should be improved by including, for instance, recommendations on mesh structure for an open catalogue of simple flows. Moreover, following the practice of the $\mathrm{V} \& \mathrm{~V}$ community (e.g. that organised by ASME), in future benchmarks more attention has to be paid to code inputs and their effects on the numerical solution of the equations.

The results obtained by each participant using the best estimate models show that the combination of mesh and modelling approach again can result in a wide spread of results, with the quality of the results not always being improved using a model selection that proved to be successful for other configurations and test conditions. For instance, the use of refined turbulence models (such as SST) and considering radiative heat transfer did not result in fully satisfactory predictions, and made evident that further investigations and validation studies are necessary to separate different sources of errors and avoid their elimination (compensating errors) in a complex model.

It is important here to stress once more the importance of blind benchmarks, because they disclose the difficulty in tackling new problems for which the modelling strategy must be derived from previous experience. This is true, we believe, in general, but it is more true for containment related problems, because the strict application of BPG is hindered by the long computation times associated with complex geometries, large volumes and long transients.

Finally, considering the specific configuration of the test on which it was based, the present benchmark revealed the (partly unexpected) difficulty to simulate a flow in presence of a simple obstruction. This observation suggests that in the future more experimental data and associated V\&V will be required to validate the codes for more complex geometries. These issues are currently addressed in the OECD/NEA project HYMERES-2 (OECD/NEA, 2017). 


\section{ACKNOWLEGMENTS}

The experiment was conducted by the Experimental thermal-hydraulic group of PSI, composed by three of the authors - R, Kapulla, S. Paranjape and D. Paladino - and G. Mignot, M. Fehlmann, L. Ryan and S. Suter. The authors gratefully acknowledge the support of all the countries participating in the OECD/NEA HYMERES project and the OECD/NEA secretariat. The authors would like to thank all the members of the Management Board and the Programme Review Group of the HYMERES project for their help in defining the test programme and evaluating the test results.

\section{REFERENCES}

Abe, S., Studer, E., Ishigaki, M., Sibamoto, Y., and Yonomoto, T. (2018) "Stratification Breakup by a Diffuse Buoyant Jet: The MISTRA HM1-1 and 1-1bis Experiments and their CFD Analysis", Nucl. Eng. Design, 331, pp. 162-175.

Abu-Romia, M.M. and Tien, C.L (1967) “Appropriate Mean Absorption Coefficients for Infrared Radiation of Gases", Journal of Heat Transfer, 89(4), 321-327.

Allelein, H.-J., Fischer, K., Vendel, J., Malet, J., Studer, E., Schwarz, S., Houkema, M., H. Paillère, H., Bentaib, A. (2007). International Standard Problem ISP-47 on Containment Thermal Hydraulics. Nuclear Energy Agency, Committee on the Safety of Nuclear Installations, Final Report NEA/CSNI/R(2007)10, 2007.

Allein, H.-J., Reinecke, E.-A., Belt, A., Broxtermann, P., and Kelm, S. (2012) “Combined Analytical and Experimental Investigations for LWR Containment Phenomena”, Nucl. Eng, Technol., 44(3), 249-260.

Andreani, M., Badillo, A., and Kapulla, R. (2016a) "Synthesis of the OECD/NEA-PSI CFD Benchmark Exercise", Nucl. Eng. Design, 299, 59-80

Andreani, M., Daqiang, Y., Gaikwad, A.J., Ganju, S., Gera., B., Grigoryev, S., Herranz, L.E., Huhtanen, R., Kanaev, A., Kelm, S., Kim, J., Nishimura, T., Schramm, B., Sharabi, M., and Paladino, D. (2016b) "Synthesis of a blind CFD benchmark exercise based on a test in the PANDA facility addressing the stratification erosion by a vertical jet in presence of a flow obstruction", 
OECD/NEA 6th Workshop on Computational Fluid Dynamics for Nuclear Reactor Safety (CFD4NRS-6), MIT, Cambridge, MA-USA, 13-15 September, 2016.

Andreani, M. and Paladino D., (Benchmark Report, 2018), prepared by PSI (with appendices contributed by AERB, FZJ and VTT) (2018) “OECD/NEA HYMERES project: synthesis of results of the benchmark on PANDA test HP1_6_2”, PSI Technical Note AN-42-17-07 Rev.1, Project report HYMERES-P-17-48, May 2018.

Bentaib, A., Meynet, N., and Bleyer, A. (2015) "Overview on Hydrogen Risk Research and Development Activities: Methodology and Open Issues”, Nucl. Eng. Technol., 47, 26-32.

Breitung, W. and Royl, P. (2000) "Procedure and Tools for Deterministic Analysis and Control of Hydrogen Behavior in Severe Accidents". Nucl. Eng. Design, 202, 249-268.

Chan, C.K. and Jones, S.C. (1997) "Gas Mixing Experiments in a Large Enclosure", Proc. of the $18^{\text {th }}$ Annual Conf. of the Canadian Nuclear Society

Choi, Y.-S., Lee, U.-J., and Park, G.-C. (2001) "Study on local hydrogen behaviors in a subcompartment of the NPP containment”, Nucl. Eng, Design, 208, 99-116.

Deri, E., Cariteau, B., and Abdo D. (2010) "Air fountains in the erosion of gaseous stratifications", Int. J. Heat and Fluid Flow, Volume 31, Issue 5, Pages 935-941.

Erkan, N., Mignot, G., Kapulla, R., Paladino, D., Zboray, R., Strassberger, H.J., Bissels, W. and Fehlmann, M. (2009) “OECD SETH-2 PANDA Test ST1_2_2 Quick-Look Report”, PSI internal technical report TM-42-09-07-0, April 2009.

Filippov, A.S., Grigoryev, S.Yu., O.V. Tarasov, O.V. (2017) "On the possible role of thermal radiation in containment thermal-hydraulics experiments by the example of CFD analysis of TOSQAN T114 air-He test", Nucl. Eng. Design, 310, 175-186.

Gupta, S. (2015) "Experimental Investigations Relevant for Hydrogen and Fission Product Issues Raised by the Fukushima Accident”, Nucl. Eng. Technol., 47, 11-25. 
Howell. J.R. (1988) "Thermal Radiation in Participating Media: The Past the Present, and Some Possible Futures", Journal Heat Transfer, 110, 1220-1229.

Huhtanen, R. (2018), Private Communication.

Kapulla, R., Mignot, G., Paranjape, S., Suter, S., Fehlmann, M., and Paladino, D. (2015a) "OECD/NEA HYMERES Project: Jet/Plume interacting with flow obstruction HP1 Series. Test Series Report”, PSI internal report TM-42-15-16, Rev.0, Project report HYMERES-P-15-26, Nov. 2015 (report restricted to project participants).

Kapulla, R., Paranjape, S., Mignot, G., Suter, S., Fehlmann, M., and Paladino D. (2015b) “OECD/NEA HYMERES Project: PANDA Tests HP1_6_0, HP1_7_0, HP1_8_0, and HP1_678_Disk Data Report”, PSI internal report TM-42-15-13 Rev. 0, Project report HYMERES-P-15-25, October 2015 (report restricted to project participants).

Karwat, H. et al. (1999) "State-of-the-Art Report on Containment Thermal-hydraulics and Hydrogen Distribution”, OECD/NEA group of experts, CSNI/R(99)-16 (1999).

Kelm, S., Ritterath, M., Prasser, H.-M., and Allelein, H.J. (2016a) "Application of the MINIPANDA Test Case 'Erosion of a Stratified Layer by a Vertical Jet' for CFD Validation”, Nucl. Eng. Design, 299, 124-135.

Kelm, S., Müller, H., and Allelein, H.-J. (2106b) "Importance of thermal radiation heat transfer modeling in containment typical flows", Paper submitted for presentation at the CFD4NRS-6, MIT, Cambridge, MA, USA, September 13-15.

Liang, R. et al., (2015) "Status Report on Hydrogen Management and Related Computer Codes“, NEA/CSNI/R(2014)8,

Lopez-Alonso E., Papini D., and Jimenez G. (2017) "Hydrogen Distribution and Passive Autocatalytic Recombiner (PAR) Mitigation in a PWR-KWU Containment Type", Annals of Nuclear Energy, 109, pp. 600-611.

Mahaffy, J., et al. (2015). Best Practice Guidelines for the Use of CFD in Nuclear Reactor Safety Applications - Revision” NEA/CSNI/R(2014)11. 
Noutsopoulos, G.C. and Yannopoulos, P.C. (1989) “Axial Dilution in Obtructed Round Buoyant Jet”,

Nishimura, T., Hoshi, H. and Hotta, A. (2015) "Current Research and Development Activities on Fission Products and Hydrogen Risk after the Accident at Fukushima Daiichi Nuclear Power Station", Nucl. Eng. Technol., 47, 1-10

OECD/NEA HYMERES-2 project (2017-2021).

OECD/NEA THAI Project (2010) "Hydrogen and Fission Product Issues Relevant for Containment Safety Assessment under Severe Accident Conditions" Final Report, 22 June 2010, Report NEA/CSNI/R(2010)3.

Paladino, D. and Dreier, J. (2012) "PANDA a Multi Purposes Integral Test Facility", Science and Technology of Nuclear Installations, 2012, Article ID 239319, doi:10.1155/2012/239319.

Paladino, D., Mignot, G., Kapulla, R., Zboray, R., Andreani, M., Tkatschenko, I., Studer, E., and Brinster, J. (2013) “OECD/SETH-2 Project: PANDA and MISTRA Experiments addressing Key Safety Issues for Water Reactor Containment", Proceedings of the $15^{\text {th }}$ International Topical Meeting on Nuclear Reactor Thermal-hydraulics (NURETH-15), paper 106, Pisa, Italy, 12-17 May 12-17, 2013.

Paranjape, S., Kapulla, R., Mignot, G. and Paladino D. (2018) “Gas Redistribution Caused by Interacting Heat Sources in the Presence of a Vertical Condenser," $12^{\text {th }}$ International Topical Meeting on Nuclear Reactor Thermal-Hydraulics, Operation and Safety (NUTHOS-12), Qingdao, China, October 14-18, 2018.

Sarikurt, F.S. and Hassan, Y.A. (2017) "Large Eddy Simulations of Erosion of a Stratified Layer by a Buoyant Jet”, Int. J. of Heat and Mass Transfer, Vol. 112, p. 354-365.

Schramm, B., Stewering, J., and Sonnenkalb M. (2017) "Einsatz von CFD-Codes für die Simulation von unfalltypischen Phänomenen im Sicherheitseinschluss: Validierung und gezielte Modellerweiterung”, Anschlussbericht RS1526, 2017 GRS-472, ISBN 978-3-946607-55-7. 
Schwarz, S., Fischer, K., Bentaib, A., Burkhardt, J., Lee, J.-J., Duspiva, J., Visser, D., Kyttala, J., Royl, P., Kim, J., Kostka, P. and Liang, R. (2011) “Benchmark on Hydrogen Distribution in a Containment based on the OECD-NEA THAI HM-2 Experiment”, Nucl. Technol., 175(3), 594-603.

Smith B.L. (2009) "Identification and Prioritization of Generic Nuclear Safety Problems Requiring CFD Analysis", Proc. 17 $7^{\text {th }}$ Int. Conf. on Nuclear Engineering (ICONE-17), Paper 75482, Brussels, Belgium, July12-16, 2009.

Studer, E., Brinster, J., Tkatschenko, I., Mignot, G., Paladino, D., and Andreani, M. (2012)

"Interaction of a light gas stratified layer with an air jet coming from below: Large scale experiments and scaling issues”, Nucl. Eng. Design, 253, 406-412. 


\section{LIST OF TABLES}

Table 1: Summary of submissions for the blind benchmark

Q 1 le 2: Overview of contributions for Step 1 of the open benchmark

Table 3: Overview of contributions for Step 2 of the open benchmark

Table 4: Overview of contributions for Step 3 of the open benchmark

Table 5: Overview of simulations performed for Step 5 of the open benchmark

Table 6: Overview of contributions for Step 4 of the open benchmark 
Table 1: Summary of submissions for the blind benchmark

\begin{tabular}{|c|c|c|c|c|}
\hline \multirow[b]{2}{*}{$\begin{array}{l}\text { Organi- } \\
\text { sation } \\
\text { Contri- } \\
\text { bution* }\end{array}$} & \multicolumn{3}{|l|}{$\mathrm{CM}$} & \multirow{2}{*}{$\begin{array}{l}\text { BEM } \\
\text { Differences from CM }\end{array}$} \\
\hline & Code & $\begin{array}{l}\text { Mesh } \\
\sum_{n+1} \text { ells } \\
3\end{array}$ & $\begin{array}{l}\text { Main } \\
\text { deviations } \\
\text { from } \\
\text { specifications }\end{array}$ & \\
\hline AERB & FLUENT 16 & 163 & - & RNG k- $\varepsilon$ \\
\hline BARC & $\begin{array}{l}\text { CFD- } \\
\text { ACE+ }\end{array}$ & 164 & $\begin{array}{l}\text { Injected steam } \\
\text { temperature }\end{array}$ & RNG k- $\varepsilon$ \\
\hline CIEMAT & FLUENT 15 & 135 & - & radiative heat transfer included \\
\hline FZJ & CFX-15 & 565 & $\begin{array}{l}\text { Constant } \\
\text { pressure }\end{array}$ & $\begin{array}{l}\text { SST, condensation and radiative heat } \\
\text { transfer included. Initial gas and wall } \mathrm{T} \\
\text { distribution. }\end{array}$ \\
\hline GRS & CFX-15 & 1412 & - & $\begin{array}{l}\text { Slightly refined mesh ( } 1.7 \text { million cells), } \\
\text { SST, radiative heat transfer included }\end{array}$ \\
\hline $\begin{array}{l}\text { IBRAE } \\
\text { IBRAE }\end{array}$ & CFX-12 & 110 & $\begin{array}{l}\text { No man-hole, } \\
\text { injected steam } \\
\text { temperature }\end{array}$ & Different code (CABARET) \\
\hline $\begin{array}{l}\text { IBRAE } \\
\text { IBRAE2 }\end{array}$ & $\begin{array}{l}\text { FLUENT } \\
14.5 .7\end{array}$ & 355 & $\begin{array}{l}\text { No man-hole, } \\
\text { plate } \\
\text { thickness }=0\end{array}$ & RSM \\
\hline KAERI & $\begin{array}{l}\text { OpenFOAM } \\
2.3 .1\end{array}$ & 158 & $\begin{array}{l}\text { Square pipe } \\
\text { and plate }\end{array}$ & \\
\hline S/NRA/R & FLUENT 15 & 655 & - & $\begin{array}{l}\text { SST, pre-conditioning phase }(\mathrm{He} \\
\text { injection) simulated }\end{array}$ \\
\hline $\begin{array}{l}\text { PSI } \\
\text { PSIF }\end{array}$ & FLUENT 15 & 560 & $\begin{array}{l}\text { Half vessels, } \\
\text { no man-hole }\end{array}$ & \\
\hline $\begin{array}{l}\text { PSI } \\
P S I G\end{array}$ & GOTHIC 8.1 & 20 & Square plate & $\begin{array}{l}\text { Initial gas and wall } \mathrm{T} \text { distribution. } \\
\text { Enhanced HTC at the walls }\end{array}$ \\
\hline SPICRI & CFX-13 & 1284 & $\begin{array}{l}\text { Initial } \mathrm{T} \text { and } \\
\mathrm{X}_{\mathrm{He}} \text { No wall } \\
\text { heat capacity }\end{array}$ & SST \\
\hline VTT & FLUENT 16 & 2872 & & \\
\hline
\end{tabular}


Table 2: Overview of contributions for Step 1 of the open benchmark

\begin{tabular}{|c|c|c|c|c|c|}
\hline $\begin{array}{l}\text { Organi- } \\
\text { sation }\end{array}$ & Code & $\begin{array}{l}\text { Cells x } 10^{3} \\
(\text { Nr. Cells for } \\
\text { Blind } \\
\text { simulations }) \\
\text { (U=unstructured } \\
\text { mesh) }\end{array}$ & $\begin{array}{l}\text { Reported } \\
\text { deviations from } \\
\text { specifications }\end{array}$ & $\begin{array}{l}\text { Size of cells } \\
\text { below } \\
\text { obstruction } \\
\text { (m) }\end{array}$ & $\begin{array}{l}\text { Equivalent } \\
\text { CPU time } \\
\text { on one } \\
\text { core } \\
\text { (h) }\end{array}$ \\
\hline AERB & $\begin{array}{l}\text { FLUENT } \\
\text { V.16 }\end{array}$ & $\begin{array}{l}240 \\
(163)\end{array}$ & $\begin{array}{l}\text { Constant } \\
\text { Pressure B.C. }\end{array}$ & $\begin{array}{l}0.03 \\
y+=40-125\end{array}$ & 1500 \\
\hline BARC & $\begin{array}{l}\text { CFD-ACE+ } \\
\text { Version } \\
2011\end{array}$ & $\begin{array}{l}690(\mathrm{U}) \\
(164)\end{array}$ & $\begin{array}{l}\text { Constant } \\
\text { Pressure B.C. }\end{array}$ & $\begin{array}{l}0.03 \\
y+=30-50\end{array}$ & 848 \\
\hline CIEMAT & $\begin{array}{l}\text { FLUENT } \\
16.2\end{array}$ & $\begin{array}{l}256(\mathrm{U}) \\
(135)\end{array}$ & $\begin{array}{l}350 \mathrm{~s} \text { transient } \\
\text { time }\end{array}$ & $\begin{array}{l}(\mathrm{V}=9.4 \mathrm{e}-7 \\
\left.\mathrm{m}^{3}\right) \\
\sqrt[3]{ } \sqrt{\mathrm{V} \sim 0.01}\end{array}$ & 24 \\
\hline FZJ & CFX-17 & $\begin{array}{l}570 \\
(565)\end{array}$ & - & $\begin{array}{l}0.005 \\
y+\sim 30\end{array}$ & 1606 \\
\hline KAERI & $\begin{array}{l}\text { OpenFOAM } \\
2.4 \mathrm{X}\end{array}$ & $\begin{array}{l}260 \\
(158)\end{array}$ & - & 0.04 & 375 \\
\hline $\mathrm{S} / \mathrm{NRA} / \mathrm{R}$ & $\begin{array}{l}\text { FLUENT } \\
\text { V15.0 }\end{array}$ & $\begin{array}{l}843(\mathrm{U}) \\
(655)\end{array}$ & - & 0.0036 & 696 \\
\hline PSI & $\begin{array}{l}\text { GOTHIC } \\
8.1\end{array}$ & $\begin{array}{l}41 \\
(20)\end{array}$ & - & $\begin{array}{l}0.01 \\
y+\sim 17-38\end{array}$ & 768 \\
\hline VTT & $\begin{array}{l}\text { FLUENT } \\
\text { V16.0 }\end{array}$ & $\begin{array}{l}2214 \\
(2872)\end{array}$ & $\begin{array}{l}\text { Enhanced wall } \\
\text { treatment }\end{array}$ & 0.0125 & 2500 \\
\hline
\end{tabular}


Table 3: Overview of contributions for Step 2 of the open benchmark

\begin{tabular}{|c|c|c|c|c|c|c|}
\hline $\begin{array}{l}\text { Organisa- } \\
\text { tion }\end{array}$ & Code & $\begin{array}{l}\text { Nr. } \\
\text { Cells } \\
\times 10^{3} \\
\text { (Step 1) }\end{array}$ & $\begin{array}{l}\text { Turbulence } \\
\text { model }\end{array}$ & Heat transfer & Annotations & $\begin{array}{l}\text { Equivale } \\
\text { nt CPU } \\
\text { time on } \\
\text { one core } \\
\text { (h) }\end{array}$ \\
\hline AERB & $\begin{array}{l}\text { FLUENT } \\
\text { V.16 }\end{array}$ & $\begin{array}{l}240 \\
(240)\end{array}$ & $\begin{array}{l}\text { Standard k- } \varepsilon \\
\text { Standard } \\
\text { wall } \\
\text { functions }\end{array}$ & $\begin{array}{l}\text { Heat loss: } \\
\text { heat flux B.C. } \\
\text { No Rad }\end{array}$ & $\begin{array}{l}\text { Constant } \\
\text { pressure B.C. } \\
\text { Lower steam } \\
\text { injection } \\
\text { temperature }\end{array}$ & 1500 \\
\hline BARC & $\begin{array}{l}\text { CFD-ACE+ } \\
\text { Version } \\
2011\end{array}$ & $\begin{array}{l}690 \\
(690)\end{array}$ & $\begin{array}{l}\text { RNG k-E } \\
\text { Non-equil. } \\
\text { wall } \\
\text { function }\end{array}$ & $\begin{array}{l}\text { Heat losses: } \\
\text { considered } \\
\text { (heat flux } \\
\text { B.C.) } \\
\text { Rad.: Discrete } \\
\text { Ordinate(DO) } \\
\text { Method }\end{array}$ & $\begin{array}{l}\text { Constant } \\
\text { pressure B.C. }\end{array}$ & 1536 \\
\hline CIEMAT & $\begin{array}{l}\text { FLUENT } \\
16.2\end{array}$ & $\begin{array}{l}256 \\
(256)\end{array}$ & $\begin{array}{l}\text { Standard k- } \varepsilon \\
\text { Standard } \\
\text { wall } \\
\text { functions }\end{array}$ & $\begin{array}{l}\text { Heat losses: } \\
\text { considered } \\
\text { No radiation }\end{array}$ & $\begin{array}{l}\text { Lower steam } \\
\text { injection } \\
\text { temperature } \\
\text { Constant } \\
\text { pressure B.C. }\end{array}$ & 44 \\
\hline FZJ & CFX-17 & $\begin{array}{l}660 \\
(570)\end{array}$ & $\begin{array}{l}\text { SST, } \\
\text { production } \\
\text { limiter for } \\
\text { TKE, Low } \\
\text { Re } \\
\text { corrections } \\
\text { Automatic } \\
\text { wall } \\
\text { treatment }\end{array}$ & $\begin{array}{l}\text { Heat losses: } \\
\text { considered } \\
\text { using } \\
\text { effective HTC } \\
\text { on outer } \\
\text { surface and } \\
\text { average steel } \\
\text { T. } \\
\text { Rad: Monte } \\
\text { Carlo method } \\
\text { with } \kappa=1 \mathrm{~m}^{-1} \\
\text { (gray gas) }\end{array}$ & 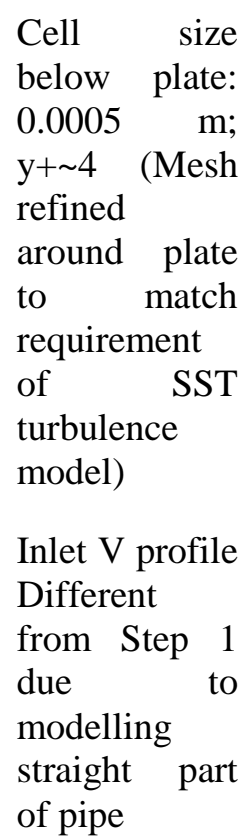 & 2932 \\
\hline
\end{tabular}




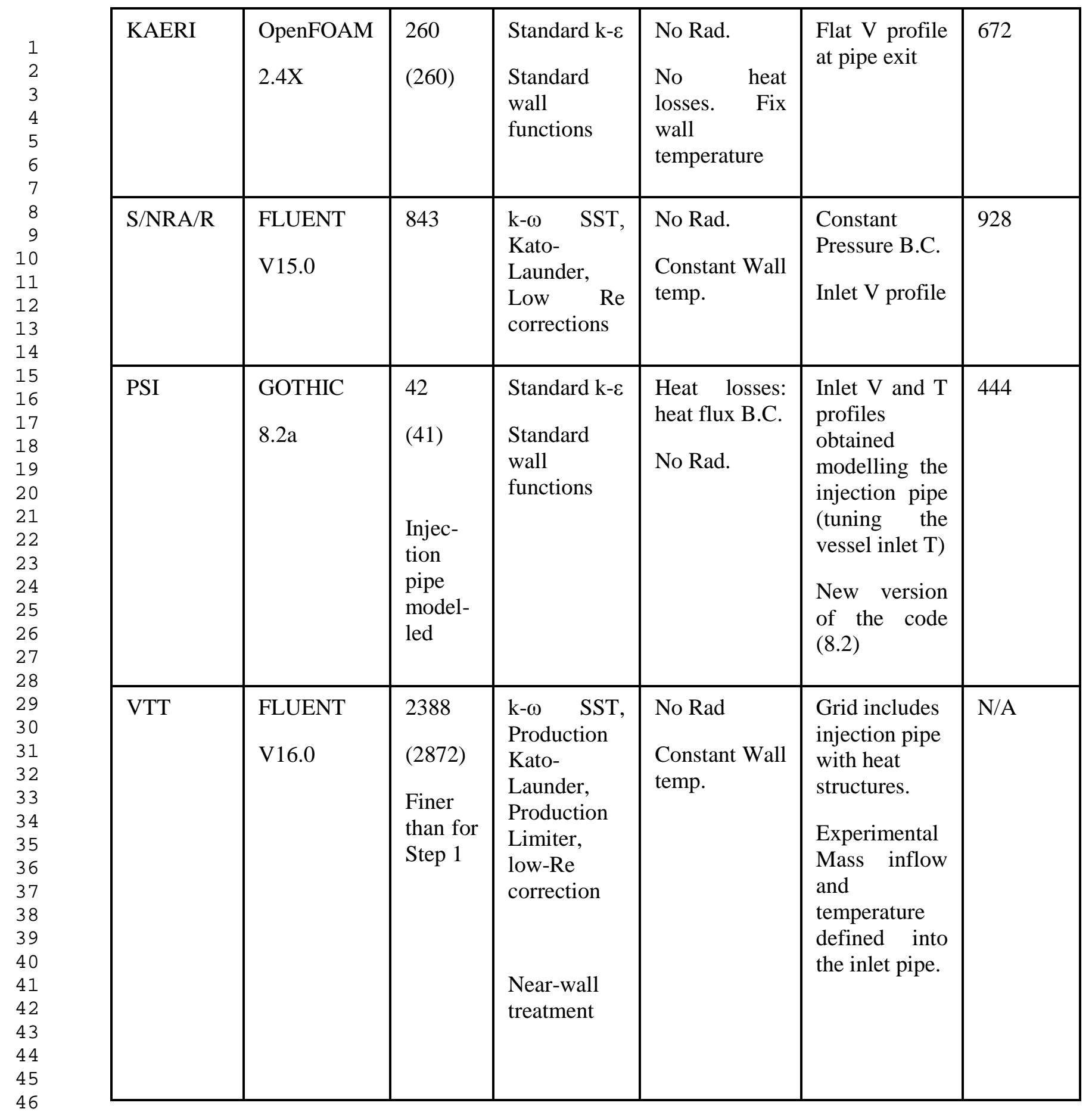


Table 4: Overview of contributions for Step 3 of the open benchmark

\begin{tabular}{|c|c|c|c|c|c|c|}
\hline $\begin{array}{l}\text { Organi- } \\
\text { sation }\end{array}$ & Code & $\begin{array}{l}\text { Nr. } \\
\text { Cells } \\
\times 10^{3} \\
\text { (used } \\
\text { for } \\
\text { steps 1 } \\
\text { and 2) }\end{array}$ & $\begin{array}{l}\text { Turbulence } \\
\text { model } \\
\text { (choice for Step } \\
\text { 2) }\end{array}$ & $\begin{array}{l}\text { Heat } \\
\text { transfer }\end{array}$ & Annotations & $\begin{array}{l}\text { Equi- } \\
\text { valent } \\
\text { CPU } \\
\text { time on } \\
\text { one } \\
\text { core (h) }\end{array}$ \\
\hline BARC & $\begin{array}{l}\text { CFD- } \\
\text { ACE+ } \\
\text { Version } \\
2011\end{array}$ & $\begin{array}{l}690 \\
(690 / \\
690)\end{array}$ & $\begin{array}{l}\text { RNG k- } \varepsilon \\
\text { Non-equil. wall } \\
\text { function } \\
\text { (same) }\end{array}$ & $\begin{array}{l}\text { Heat } \\
\text { losses: } \\
\text { considered } \\
\text { (heat flux } \\
\text { B.C.) } \\
\text { Rad: DO } \\
\text { method }\end{array}$ & $\begin{array}{l}\text { Constant } \\
\text { pressure B.C. }\end{array}$ & 904 \\
\hline CIEMAT & $\begin{array}{l}\text { FLUENT } \\
17.1\end{array}$ & $\begin{array}{l}429 \\
(256 / \\
256)\end{array}$ & $\begin{array}{l}\text { SST k- } \omega \\
\text { Standard wall } \\
\text { functions }\end{array}$ & $\begin{array}{l}\text { Heat } \\
\text { losses: } \\
\text { Considered } \\
\text { Rad: DO } \\
\text { method }\end{array}$ & $\begin{array}{l}\text { Mesh refined } \\
\text { Min/Maximum } \\
\text { volume }\left(\mathrm{m}^{3}\right) \text { : } \\
1.1424 \mathrm{e}-07 / \\
1.4587 \mathrm{e}-02 \\
\text { cell below plate } \\
\approx 7.3 \mathrm{e}-07 \\
\text { New version of } \\
\text { the code used }\end{array}$ & 412 \\
\hline FZJ & CFX-17 & $\begin{array}{l}660 \\
(570 / \\
660)\end{array}$ & $\begin{array}{l}\text { SST } \\
\text { Automatic wall } \\
\text { treatment. } \\
\text { (same) }\end{array}$ & $\begin{array}{l}\text { Radiative } \\
\text { heat } \\
\text { transfer } \\
\text { considered. } \\
\text { Gray gas. } \\
\text { Absorption } \\
\text { coefficient: } \\
1 \mathrm{~m}^{-1}\end{array}$ & $\begin{array}{l}\text { Cell size below } \\
\text { plate: } 0.0005 \\
\text { m; y+ 4 } \\
\text { (same as for } \\
\text { Step 2) }\end{array}$ & 2882 \\
\hline S/NRA/R & $\begin{array}{l}\text { FLUENT } \\
\text { V15.0 }\end{array}$ & $\begin{array}{l}843 \\
(843 / \\
843)\end{array}$ & $\begin{array}{l}\text { SST,Kato- } \\
\text { Launder, low } \\
\text { Re corrections } \\
\text { (same) }\end{array}$ & $\begin{array}{l}\text { No Rad. } \\
\text { Constant } \\
\text { Wall temp. }\end{array}$ & $\begin{array}{l}\text { Cell size below } \\
\text { plate: } 0.0036 \\
\text { m. } \\
\text { Cond./Rad. No } \\
\text { Inlet V profile }\end{array}$ & 480 \\
\hline
\end{tabular}




\begin{tabular}{|c|c|c|c|c|c|c|c|}
\hline $\begin{array}{l}1 \\
2 \\
3 \\
4 \\
5 \\
6 \\
7 \\
8 \\
9\end{array}$ & PSI & $\begin{array}{l}\text { GOTHIC } \\
8.2\end{array}$ & $\begin{array}{l}42 \\
(41 / 42)\end{array}$ & $\begin{array}{l}\text { Standard k- } \varepsilon \\
\text { Standard wall } \\
\text { functions } \\
\text { (same) }\end{array}$ & $\begin{array}{l}\text { Heat } \\
\text { losses: heat } \\
\text { flux B.C. } \\
\text { No } \\
\text { Radiation }\end{array}$ & & 755 \\
\hline $\begin{array}{l}10 \\
11 \\
12 \\
13 \\
14 \\
15 \\
16 \\
17 \\
18 \\
19 \\
20 \\
21 \\
22 \\
23 \\
24 \\
25\end{array}$ & VTT & $\begin{array}{l}\text { FLUENT } \\
\text { V16.0 }\end{array}$ & $\begin{array}{l}2311 \\
(2214 \\
2378)\end{array}$ & $\begin{array}{l}\text { SST k- } \omega \text {, } \\
\text { Production } \\
\text { Kato-Launder, } \\
\text { Production } \\
\text { Limiter, } \\
\text { low Re } \\
\text { corrections } \\
\text { Near wall } \\
\text { treatment } \\
\text { (same) }\end{array}$ & $\begin{array}{l}\text { No } \\
\text { Radiation } \\
\text { Constant } \\
\text { Wall temp. }\end{array}$ & $\begin{array}{l}\text { Grid includes } \\
\text { injection pipe } \\
\text { with heat } \\
\text { structures. } \\
\text { Experimental } \\
\text { Mass inflow } \\
\text { and } \\
\text { temperature } \\
\text { defined into the } \\
\text { inlet pipe }\end{array}$ & N/A \\
\hline
\end{tabular}


Table 5: Overview of simulations performed for Step 5 of the open benchmark

\begin{tabular}{|c|c|c|c|c|c|c|}
\hline $\begin{array}{l}\text { Organi- } \\
\text { sation }\end{array}$ & Code & $\begin{array}{l}\text { Nr. } \\
\text { Cells } \\
\times 10^{3} \\
\\
\text { (Steps } \\
2 / 3)\end{array}$ & $\begin{array}{l}\text { Turbulence } \\
\text { model } \\
\text { (Steps } 2 / 3 \text { ) }\end{array}$ & $\begin{array}{l}\text { Heat } \\
\text { transfer }\end{array}$ & Annotations & $\begin{array}{l}\text { Equivalent } \\
\text { CPU time } \\
\text { on one } \\
\text { core }(h)\end{array}$ \\
\hline AERB & $\begin{array}{l}\text { FLUENT } \\
\text { V.16 }\end{array}$ & $\begin{array}{l}895 \\
(240)\end{array}$ & $\begin{array}{l}\text { Realizable k- } \varepsilon \\
\text { Non- } \\
\text { equilibrium } \\
\text { wall functions } \\
\text { (Standard k-E, } \\
\begin{array}{l}\text { standard wall } \\
\text { functions)) }\end{array}\end{array}$ & $\begin{array}{l}\text { Heat loss: } \\
\text { heat flux } \\
\text { B.C. } \\
\text { No } \\
\text { Radiation }\end{array}$ & $\begin{array}{l}\text { Straight part } \\
\text { of Injection } \\
\text { pipe } \\
\text { modelled to } \\
\text { get velocity } \\
\text { profile. } \\
\text { Constant } \\
\text { pressure B.C. }\end{array}$ & 2160 \\
\hline FZJ & CFX-16.1 & $\begin{array}{l}560 \\
(660)\end{array}$ & $\begin{array}{l}\text { SST } \\
\text { Automatic wall } \\
\text { treatment }\end{array}$ & $\begin{array}{l}\text { Heat loss: } \\
\text { heat flux } \\
\text { B.C. } \\
\text { Radiative } \\
\text { heat } \\
\text { transfer } \\
\text { considered. } \\
\text { Gray gas. } \\
\text { Absorption } \\
\text { coefficient: }^{1} \text { - }^{1}\end{array}$ & $\begin{array}{l}\text { Straight part } \\
\text { of Injection } \\
\text { pipe } \\
\text { modelled to } \\
\text { get velocity } \\
\text { profile (w/o } \\
\text { heat transfer) } \\
\text { Constant } \\
\text { pressure B.C. }\end{array}$ & 2660 \\
\hline PSI & $\begin{array}{l}\text { GOTHIC } \\
8.2 \mathrm{a}\end{array}$ & 42 & $\begin{array}{l}\text { Standard k- } \varepsilon \\
\text { Standard wall } \\
\text { functions }\end{array}$ & $\begin{array}{l}\text { Heat } \\
\text { losses: heat } \\
\text { flux B.C. } \\
\text { No } \\
\text { Radiation }\end{array}$ & $\begin{array}{l}\text { Same mesh } \\
\text { as for other } \\
\text { steps } \\
\text { (including } \\
\text { denser mesh } \\
\text { at } 5 \mathrm{~m} \text { ) }\end{array}$ & 755 \\
\hline SPICRI & $\begin{array}{l}\cos C Y C A S \\
1.0\end{array}$ & 5.5 & $\begin{array}{l}\text { Standard k- } \varepsilon \\
\text { Standard wall } \\
\text { functions }\end{array}$ & $\begin{array}{l}\text { Wall heat } \\
\text { transfer } \\
\text { considered }\end{array}$ & $\begin{array}{l}\text { Axis- } \\
\text { symmetric } \\
\text { model }\end{array}$ & 27.9 \\
\hline
\end{tabular}


Table 6: Overview of contributions for Step 4 of the open benchmark

\begin{tabular}{|c|c|c|c|c|c|c|}
\hline $\begin{array}{l}\text { Organi- } \\
\text { sation }\end{array}$ & Code & $\begin{array}{l}\text { Nr. } \\
\text { Cells } \\
\times 10^{3} \\
\text { (Steps } \\
1,2,3 \\
\text { and 5) }\end{array}$ & $\begin{array}{l}\text { Turbulence } \\
\text { model } \\
\text { (steps 2,3,5) }\end{array}$ & $\begin{array}{l}\text { Heat } \\
\text { transfer }\end{array}$ & Annotations & $\begin{array}{l}\text { Equiva- } \\
\text { lent } \mathrm{CPU} \\
\text { time on } \\
\text { one core } \\
\text { (h) }\end{array}$ \\
\hline AERB & $\begin{array}{l}\text { FLUENT } \\
\text { V16.1 }\end{array}$ & $\begin{array}{l}899 \\
(240, \\
240, \\
\text { N/A, } \\
894)\end{array}$ & $\begin{array}{l}\text { Realizable } \\
\text { k- } \varepsilon \\
\text { (standard) } \\
\text { Non-equil. } \\
\text { wall } \\
\text { function } \\
\text { (standard) }\end{array}$ & $\begin{array}{l}\text { Heat losses: } \\
\text { heat flux } \\
\text { B.C. } \\
\text { No Rad. }\end{array}$ & $\begin{array}{l}\text { Straight part of } \\
\text { Injection pipe } \\
\text { modelled to get } \\
\text { velocity profile. } \\
\text { Constant } \\
\text { pressure B.C. }\end{array}$ & 1730 \\
\hline BARC & $\begin{array}{l}\text { CFD- } \\
\text { ACE+ } \\
\text { Version } \\
2011\end{array}$ & $\begin{array}{l}690 \\
(690 . \\
690, \\
690, \\
N / A)\end{array}$ & $\begin{array}{l}\text { RNG k- } \varepsilon \\
\text { Non-equil. } \\
\text { wall } \\
\text { function } \\
\text { (same) }\end{array}$ & $\begin{array}{l}\text { Heat losses: } \\
\text { heat flux } \\
\text { B.C. } \\
\text { No Cond. } \\
\text { Rad: DO } \\
\text { method }\end{array}$ & $\begin{array}{l}\text { Cell size below } \\
\text { plate: } 0.03 \mathrm{~m} \text {; } \\
\mathrm{y}+\text { between } 30 \\
\text { and } 50 . \\
\text { Constant } \\
\text { pressure B.C. }\end{array}$ & 1536 \\
\hline CIEMAT & $\begin{array}{l}\text { FLUENT } \\
17.1\end{array}$ & $\begin{array}{l}133 \\
(260, \\
260, \\
420, \\
N / A)\end{array}$ & $\begin{array}{l}\text { Standard k-E } \\
\text { Standard } \\
\text { wall } \\
\text { functions } \\
\text { (SST) }\end{array}$ & $\begin{array}{l}\text { Heat losses: } \\
\text { Considered } \\
\text { No Cond. } \\
\text { Radiation: } \\
\text { DO method }\end{array}$ & $\begin{array}{l}\text { Submitted } \\
\text { results using } \\
\text { the same model } \\
\text { as for BEM } \\
\text { blind } \\
\text { simulation }\end{array}$ & $\begin{array}{l}188 \\
1920 \text { for } \\
\text { the BEM } \\
\text { blind } \\
\text { simulation }\end{array}$ \\
\hline FZJ & CFX-17 & $\begin{array}{l}660 \\
(570, \\
660, \\
660, \\
550)\end{array}$ & $\begin{array}{l}\text { SST } \\
\text { Automatic } \\
\text { wall } \\
\text { treatment } \\
\\
\text { (same) }\end{array}$ & $\begin{array}{l}\text { Radiative } \\
\text { heat } \\
\text { transfer: } \\
\text { Monte } \\
\text { Carlo. Gray } \\
\text { gas, } \\
\text { absorption } \\
\text { coefficient: } \\
1 \mathrm{~m}^{-1}\end{array}$ & $\begin{array}{l}\text { Cell size below } \\
\text { plate: } 0.0005 \\
\text { m; } y+\sim 4\end{array}$ & 5551 \\
\hline
\end{tabular}




\begin{tabular}{|c|c|c|c|c|c|c|}
\hline GRS & CFX-17 & 7000 & $\begin{array}{l}\text { SST, } \\
\text { Including } \\
\text { buoyancy } \\
\text { turbulence } \\
\text { terms } \\
\text { automatic } \\
\text { wall } \\
\text { treatment }\end{array}$ & $\begin{array}{l}\text { Radiative } \\
\text { heat } \\
\text { transfer: } \\
\text { DTM (64 } \\
\text { rays), gray } \\
\text { gas, } \\
\text { absorption } \\
\text { coefficient } \\
\sim 1 \mathrm{~m}^{-1} \text { (from } \\
\text { RS1500 } \\
\text { correlation) }\end{array}$ & $\begin{array}{l}\text { Straight part of } \\
\text { injection pipe } \\
\text { modelled to get } \\
\text { velocity profile }\end{array}$ & 17700 \\
\hline S/NRA/R & $\begin{array}{l}\text { FLUENT } \\
\text { V15.0 }\end{array}$ & $\begin{array}{l}843 \\
(843, \\
843, \\
843, \\
N / A)\end{array}$ & $\begin{array}{l}\text { SST, } \\
\text { Kato- } \\
\text { Launder, } \\
\text { low Re } \\
\text { corrections } \\
\text { no wall } \\
\text { treatment } \\
\text { (same) }\end{array}$ & $\begin{array}{l}\text { No Cond. } \\
\text { No Rad. } \\
\text { Constant } \\
\text { Wall temp. }\end{array}$ & $\begin{array}{l}\text { Cell size below } \\
\text { plate: } 0.0036 \\
\mathrm{~m} . \\
\text { Constant } \mathrm{P} \\
\text { Inlet V profile }\end{array}$ & 208 \\
\hline PSI & $\begin{array}{l}\text { GOTHIC } \\
8.2\end{array}$ & $\begin{array}{l}42 \\
(41.4 \\
2,42,4 \\
2)\end{array}$ & $\begin{array}{l}\text { Standard k- } \varepsilon \\
\text { Standard } \\
\text { wall } \\
\text { functions } \\
\text { (same) }\end{array}$ & $\begin{array}{l}\text { Heat losses: } \\
\text { heat flux } \\
\text { B.C. } \\
\text { Rad: not } \\
\text { considered }\end{array}$ & $\begin{array}{l}\text { Contrarily to } \\
\text { BEM simulation for } \\
\text { the blind } \\
\text { benchmark, the } \\
\text { low initial } \\
\text { values of the } \\
\text { wall } \\
\text { temperature at } \\
\text { the top of the } \\
\text { dome were not } \\
\text { used for a large } \\
\text { part of the } \\
\text { dome, and } \\
\text { Standard } \\
\text { convective } \\
\text { HTC } \\
\text { correlation } \\
\text { were used (no } \\
\text { enhancement } \\
\text { factor) }\end{array}$ & 768 \\
\hline
\end{tabular}




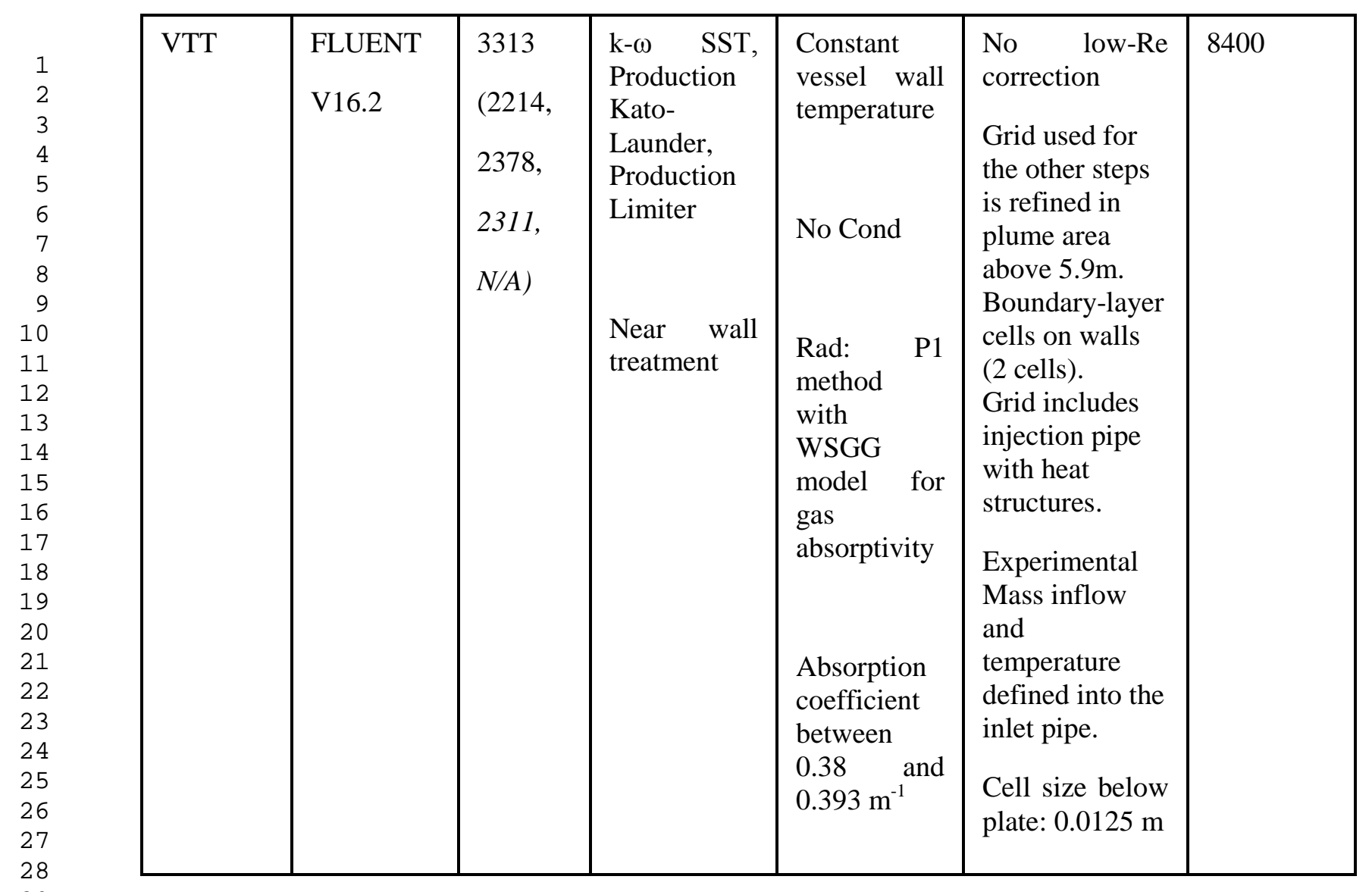




\section{LIST OF FIGURES}

Figure 1: Cutaway drawing of the vessels (left), configuration for the experiment (middle), and initial helium concentration vertical profile in Vessel 1 (right).

Figure 2: Time histories of helium concentrations (left) and gas temperatures (right) at various elevations along axis of Vessel 1.

Figure 3: Blind benchmark: Erosion progression (right) calculated with the CM and time history (left) of gas temperature at position B20 $(\mathrm{z}=7500 \mathrm{~mm})$, shown as an example. The dashed line shows how the erosion time at each elevation (e.g. at the elevation of position B20) is derived.

Figure 4: Blind benchmark: Helium concentration time histories at elevation A (left) and B (right) calculated with the "Common Model" (CM)

Figure 5: Blind benchmark: Gas temperature horizontal profile at level $\mathrm{G}$ at $300 \mathrm{~s}$ (left) and at level $\mathrm{H}$ at $150 \mathrm{~s}$ (right) calculated with the CM. Square marks show the experimental data.

Figure 6: Blind benchmark: Erosion progression (left) and helium concentration time history at Level $\mathrm{B}$ (right) calculated with the BEM.

Figure 7: (top) vertical section (plane $315^{\circ}-135^{\circ}$ ) and horizontal section of Vessel 1 showing the locations of the concentration and gas temperature measurements and the Field of View (FOV) for PIV measurements used for HP1_6_2 (Vessel 2 and IP are not shown); (bottom) position of the combined PIV window used for Tests HP1_X_0.

Figure 8: Open benchmark, Step 1: Average experimental flow field (left) measured in Test HP1_6_0, and vertical velocity profiles used in the comparisons with calculated results (right).

Figure 9: Open benchmark, Step 1: Axial profile of the vertical velocity (left) and vertical profile of the maximum vertical velocity (right).

Figure 10: Open benchmark, Step 1: Measured distribution of Turbulent Kinetic Energy (left) and comparison between experimental and calculated axial profile

Figure 11: Open benchmark, Step 1: Difference of the maximum velocity in the two half planes (left) and position (Y2) of the full jet recovery (right).

Figure 12: Open benchmark, Step 1: Time history of the minimum vertical velocity (left) and elevation (Y1) of the top of the recirculation zone (right). 
Figure 13: Open benchmark, Step 1: Horizontal profiles of the vertical velocity at the elevation of the middle of the recirculation zone (left) and at an elevation in the flow developed region (right).

Figure 14: Open benchmark, Step 1: Horizontal profiles of the TKE at the elevation of the middle of the recirculation zone (left) and at an elevation in the flow developed region (right).

Figure 15: Open benchmark, Step 2: Average experimental flow field (left) measured in Test HP1_7_0, and vertical velocity profiles used in the comparisons with calculated results (right).

Figure 16: Open benchmark, Step 2: Axial profile of the vertical velocity (left) and vertical profile of the maximum vertical velocity (right).

Figure 17: Open benchmark, Step 2: Temperature radial profile at $6.276 \mathrm{~m}$ (left) and axial temperature distribution (right).

Figure 18: Open benchmark, Step 3: Axial velocity and maximum velocity vertical profiles for Step 3, compared with results for Step 1.

Figure 19: Open benchmark, Step 3: Axial temperature profile and horizontal profile at $6.3 \mathrm{~m}$.

Figure 20: Open benchmark, Step 5: Configuration for SETH-2 test ST1_2_2, and important initial and boundary conditions of test ST1_2 and repetition test ST1_2_2 compared with those used for Test HP1_6_2.

Figure 21: Open benchmark, Step 5: Progression of erosion along the axis in test ST1_2_2, using gas temperature rise times.

Figure 22: Open benchmark, Step 5: Helium concentration time histories at various elevations along the axis of Vessel 1.

Figure 23: Open benchmark, Step 5: Vertical velocity axial profile (left) and horizontal profile at $\mathrm{z}=5003 \mathrm{~mm}(1 \mathrm{~m}$ above pipe exit)

Figure 24: Open benchmark, Step 5: Axial temperature profile at two times.

Figure 25: Open benchmark, Step 5: horizontal profiles at $6.3 \mathrm{~m}$ at $150 \mathrm{~s}$ (before mixing) and $300 \mathrm{~s}$ (after mixing)

Figure 26: Open benchmark, Step 5: horizontal profiles at $6.93 \mathrm{~m}$ at $250 \mathrm{~s}$ (during mixing or immediately after) and $500 \mathrm{~s}$ (after mixing)

Figure 27: Open benchmark, Step 4: Erosion progression calculated in the open phase (left), compared with best estimate results contributed to the blind benchmark. 
Figure 28: Open benchmark, Step 4: Helium concentration time history at $\mathrm{z}=6.48 \mathrm{~m}$ calculated in the open phase (left), compared with best estimate results contributed to the blind benchmark.

Figure 29: Open benchmark, Step 4: Example of results obtained using the model for radiation or neglecting it: helium concentration time histories calculated at 8 m (left, GRS, Schramm et al., 2017) and at $7.5 \mathrm{~m}$ (right, VTT, Huhtanen, 2018).

Figure 30: Open benchmark, Step 4: Vertical velocity averaged axial and horizontal profiles in the PIV window.

Figure 31: Open benchmark, Step 4: Axial gas temperature distributions at two times

Figure 32: Open benchmark, Step 4: Gas temperature horizontal profiles at various elevations and two times.

Figure 33: Open benchmark, Step 4: Gas temperature time histories at two positions (left: on the axis; right: at $570 \mathrm{~mm}$ from the wall) at $6.5 \mathrm{~m}$.

Figure 34: Open benchmark, Step 4: Off-axis (r: $\pm 325 \mathrm{~mm})$ gas temperature time histories at two positons below the initial bottom of the helium layer (z: 5301 and $4000 \mathrm{~mm}$, respectively) 

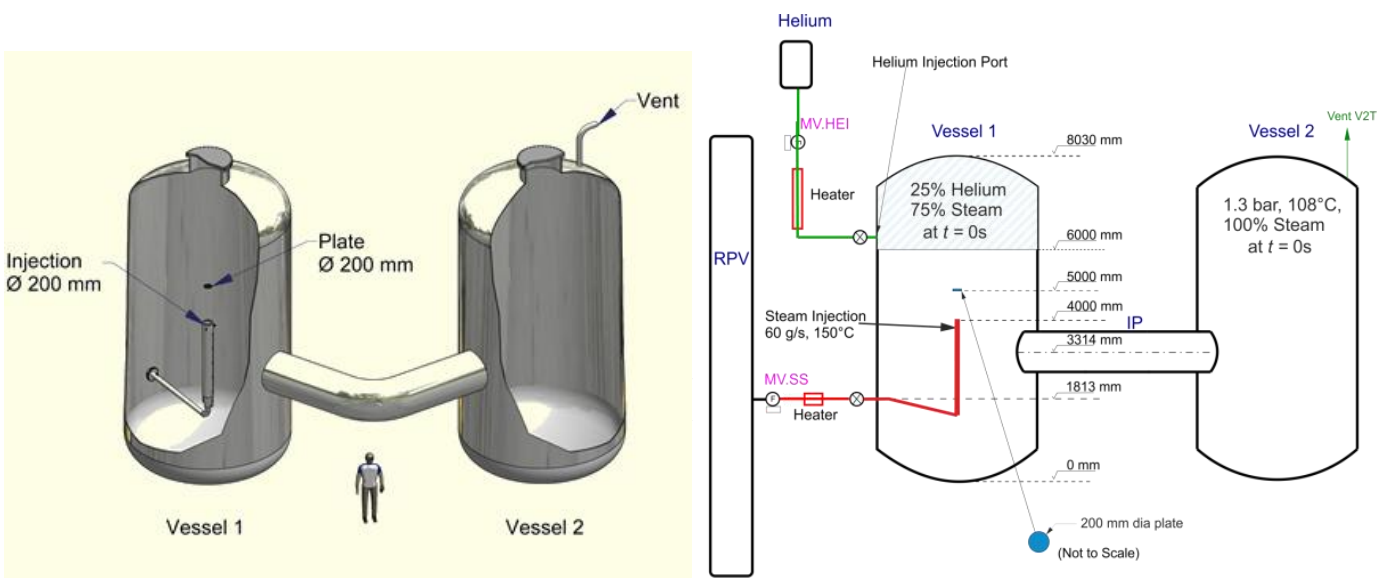

Thure 1: Cutaway drawing of the vessels (left), configuration for the experiment (middle), and initial helium concentration vertical profile in Vessel 1 (right).
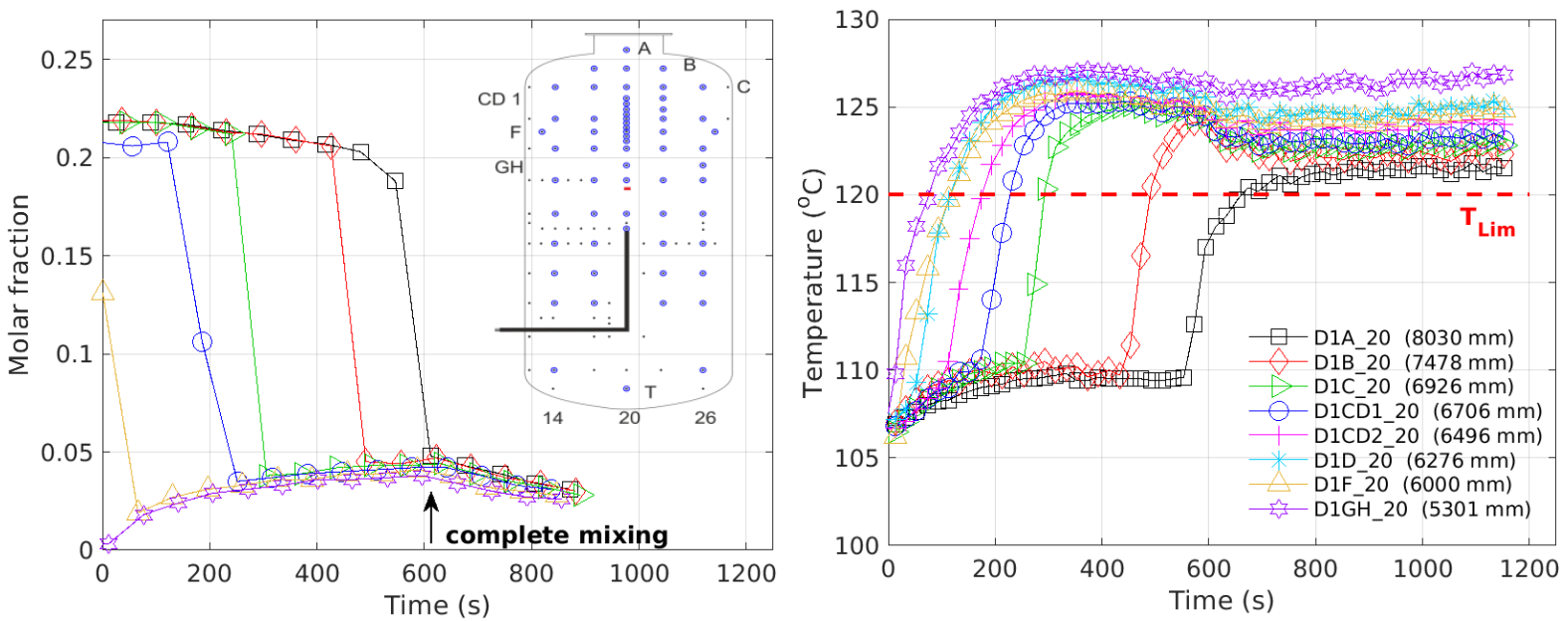

Figure 2: Time histories of helium concentrations (left) and gas temperatures (right) at various elevations along axis of Vessel 1.
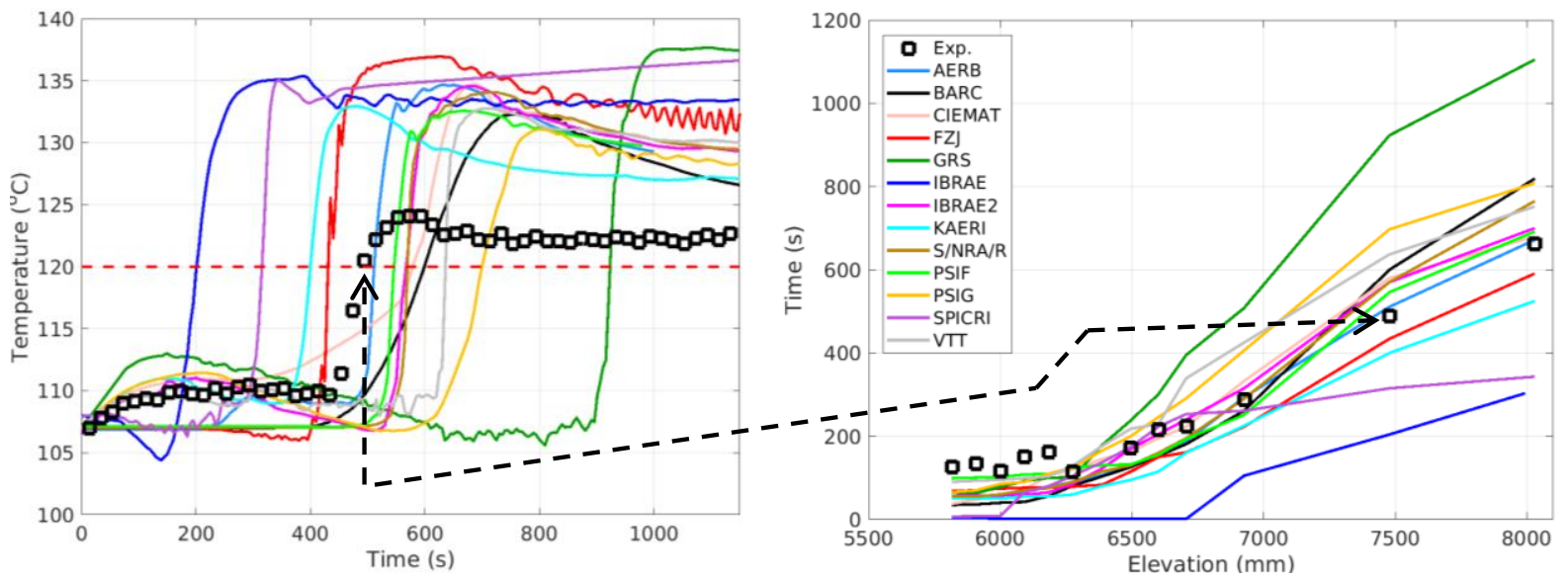

Figure 3: Blind benchmark: erosion progression (right) calculated with the CM and time history (left) of gas temperature at position B20 (z=7500 mm), shown as an example. The dashed line shows how the erosion time at each elevation (e.g. at the elevation of position B20) is derived. 

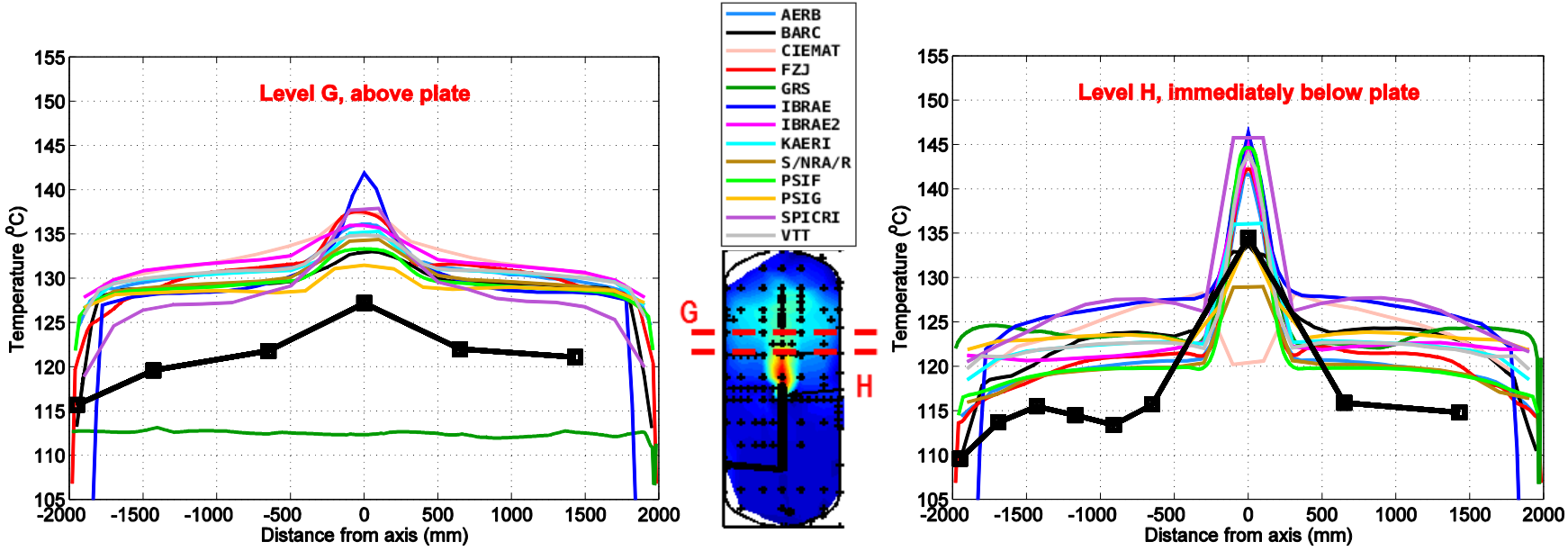

Figure 5: Blind benchmark: Gas temperature horizontal profile at level $\mathrm{G}$ at $300 \mathrm{~s}$ (left) and at level $\mathrm{H}$ at $150 \mathrm{~s}$ (right) calculated with the CM. Square marks show the experimental data.
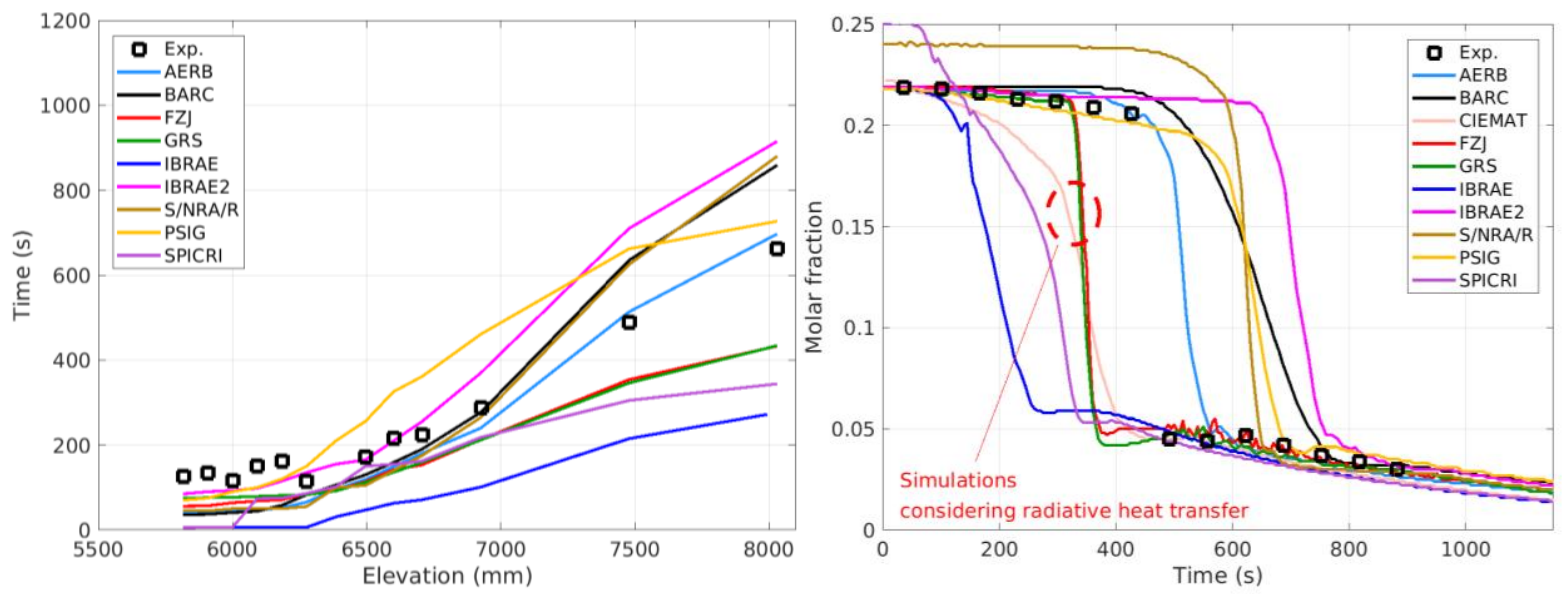

Figure 6: Blind benchmark: Erosion progression (left) and helium concentration time history at Level B (right) calculated with the BEM. 

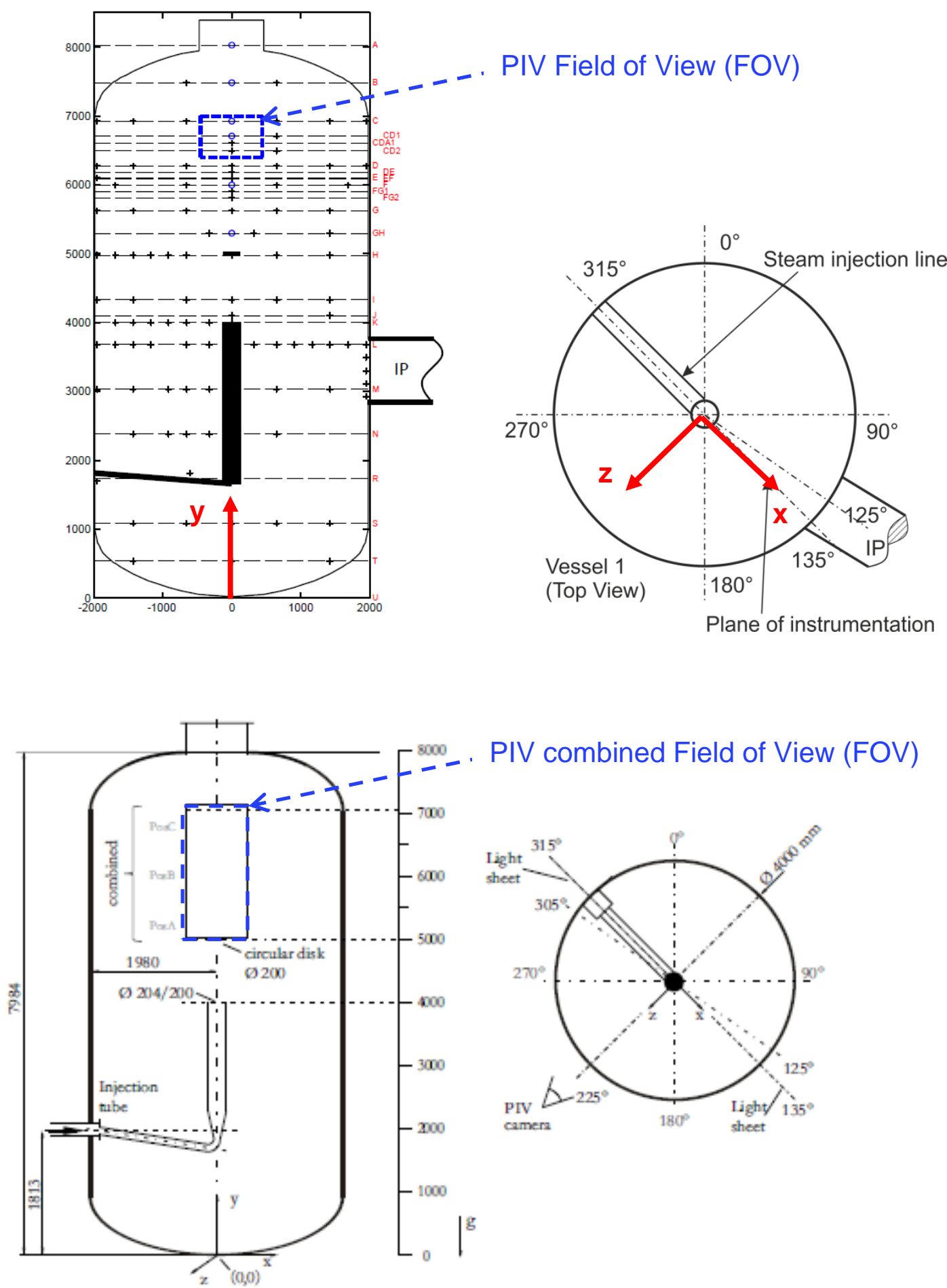

Figure 7: (top) vertical section (plane $315^{\circ}-135^{\circ}$ ) and horizontal section of Vessel 1 showing the locations of the concentration and gas temperature measurements and the Field of View (FOV) for PIV measurements used for HP1_6_2 (Vessel 2 and IP are not shown); (bottom) position of the combined PIV window used for Tests HP1_X_0. 

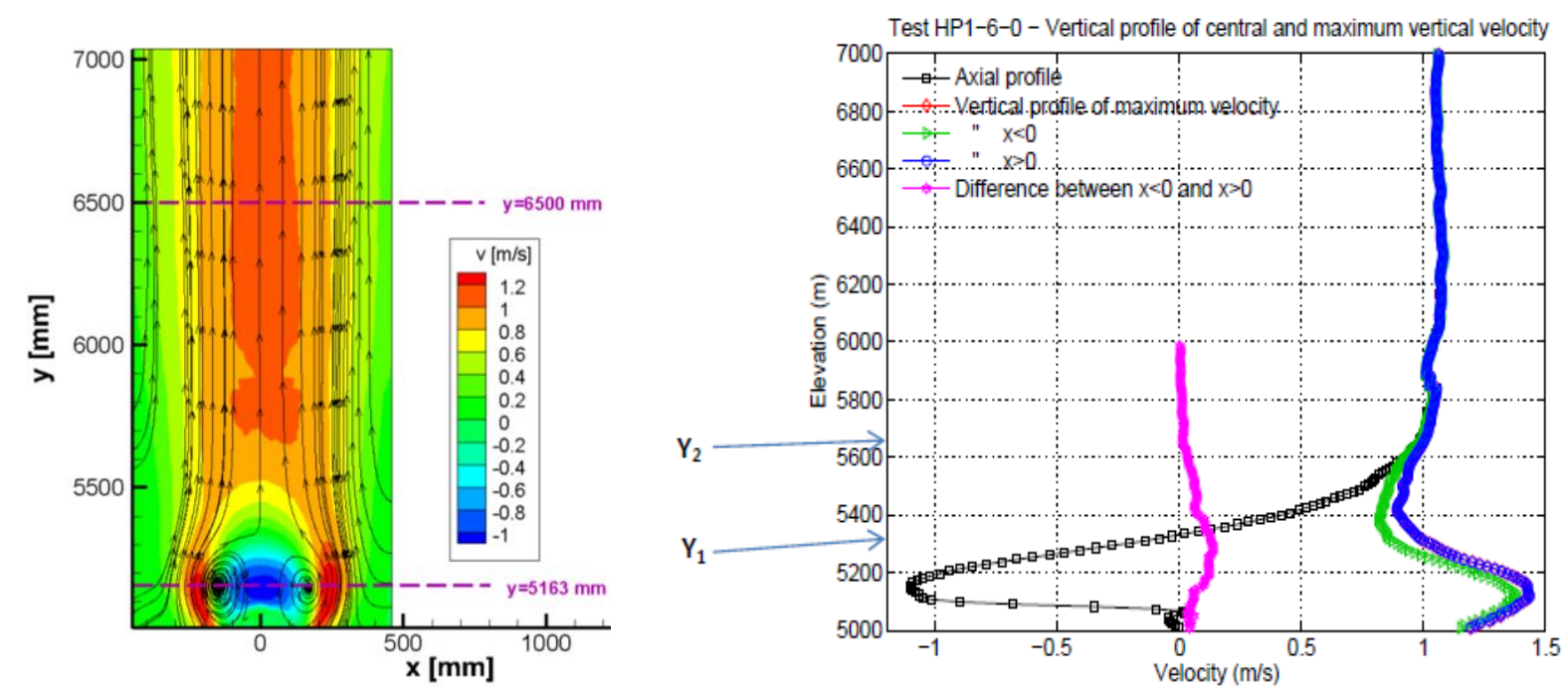

Figure 8: Open benchmark, Step 1: Average experimental flow field (left) measured in Test HP1_6_0, and vertical velocity profiles used in the comparisons with calculated results (right).
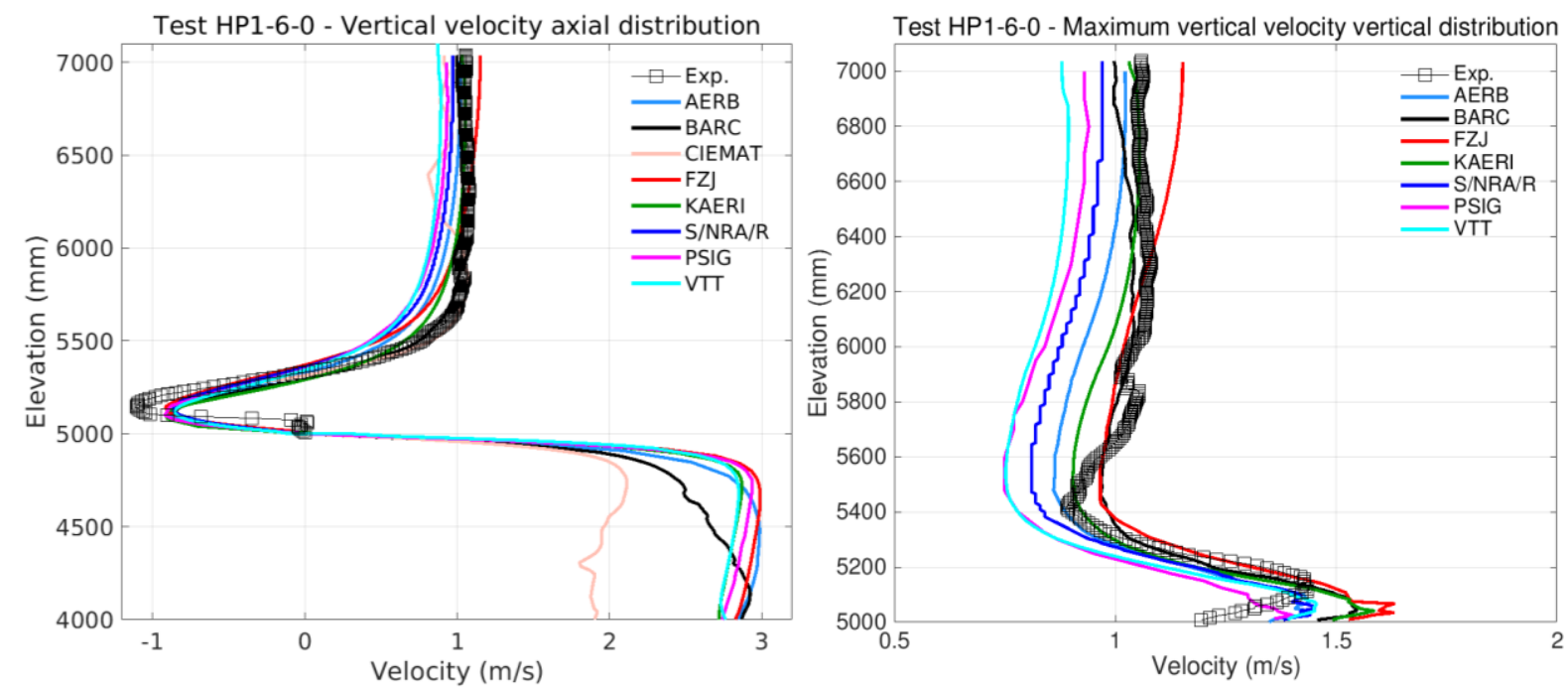

Figure 9: Open benchmark, Step 1: Axial profile of the vertical velocity (left) and vertical profile of the maximum vertical velocity (right). 

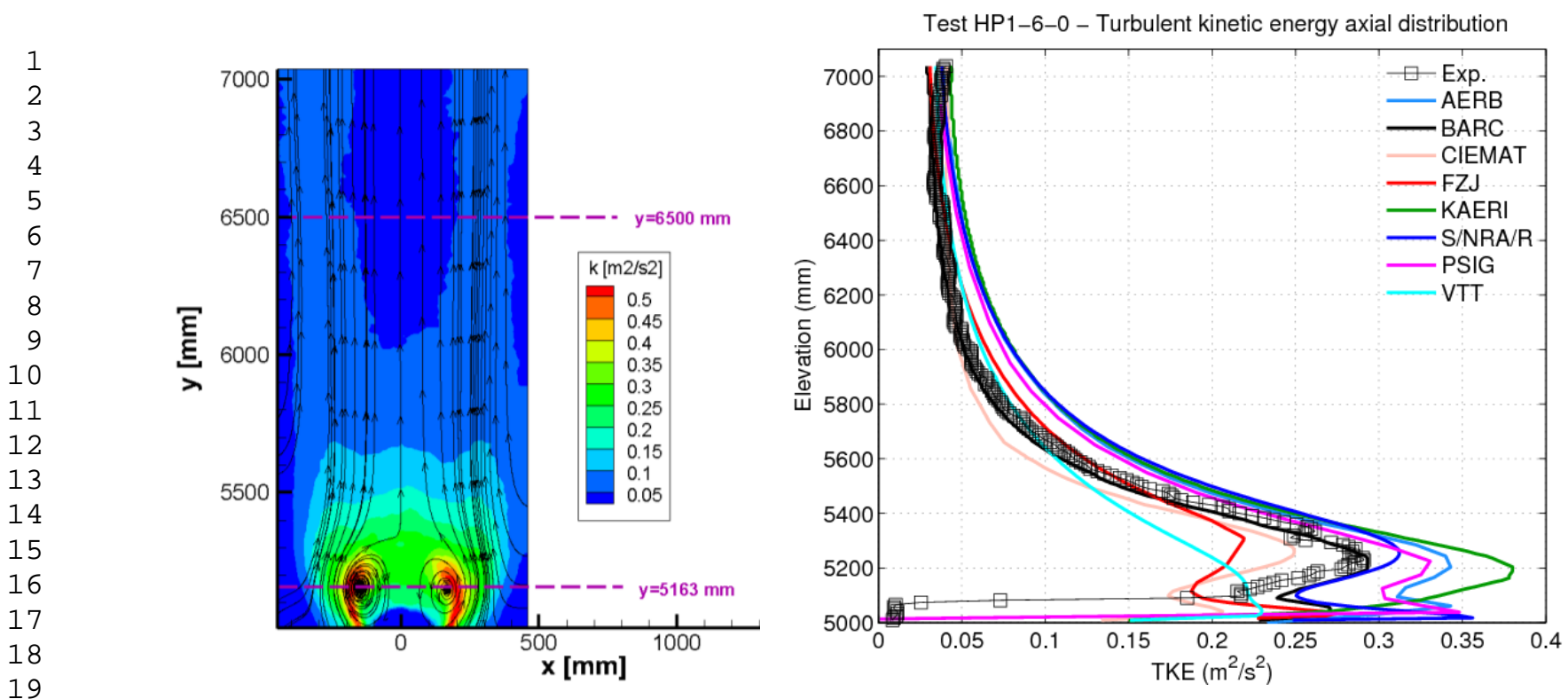

Figure 10: Open benchmark, Step 1: Measured distribution of Turbulent Kinetic Energy (left) and comparison between experimental and calculated axial profile.
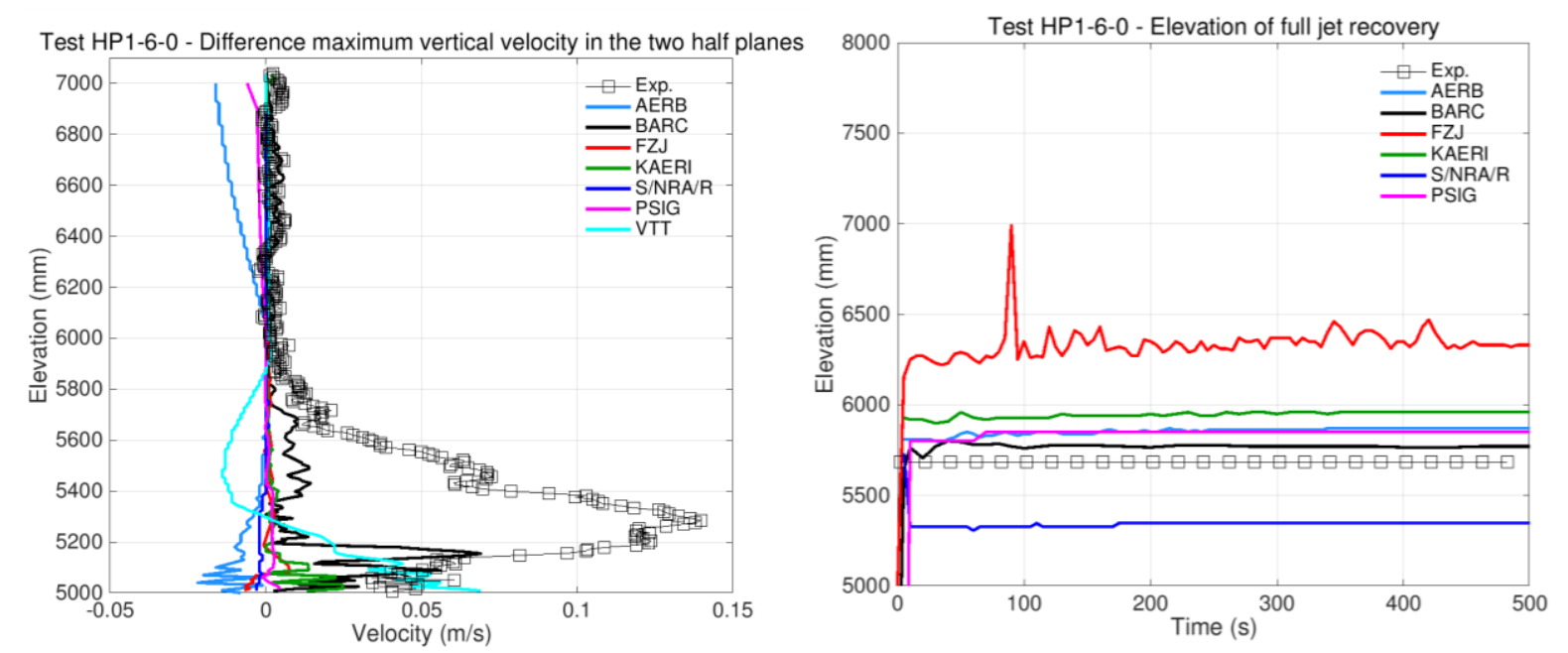

Figure 11: Open benchmark, Step 1: Difference of the maximum velocity in the two half planes (left) and position (Y2) of the full jet recovery (right). 

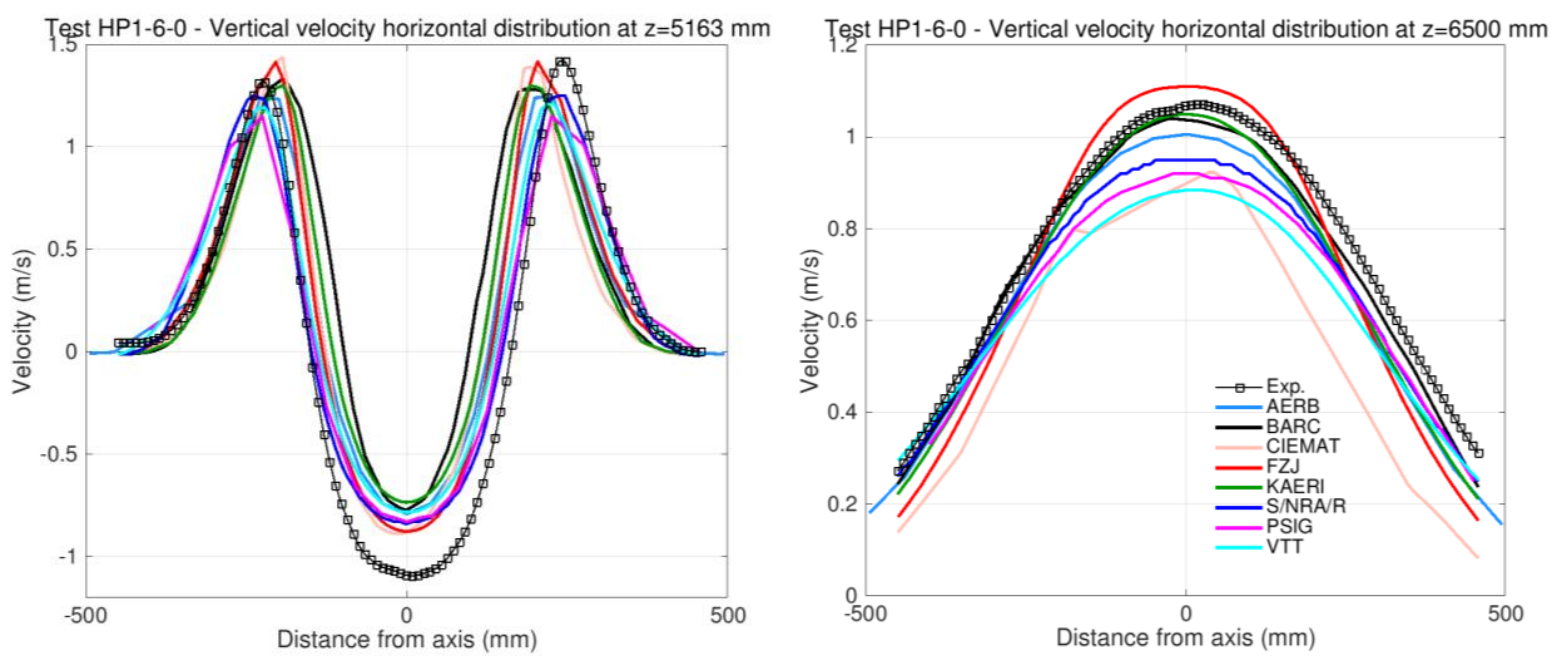

Figure 13: Open benchmark, Step 1: Horizontal profiles of the vertical velocity at the elevation of the middle of the recirculation zone (left) and at an elevation in the flow developed region (right). 

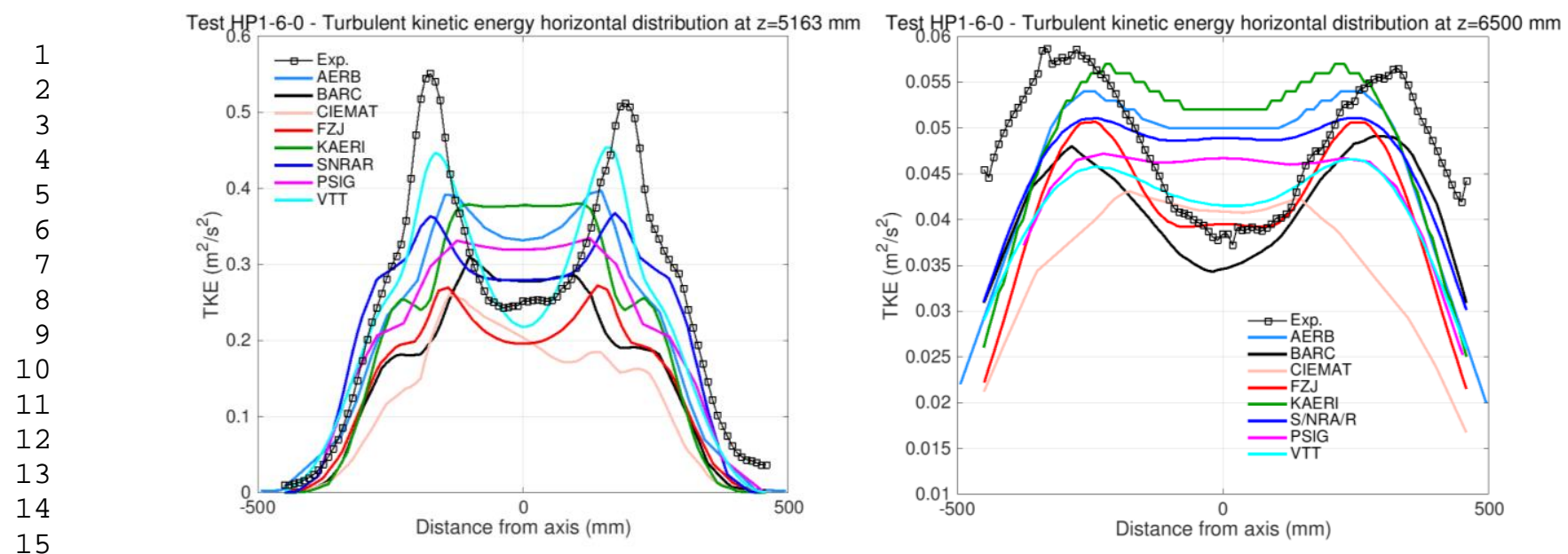

Figure 14: Open benchmark, Step 1: Horizontal profiles of the TKE at the elevation of the middle of the recirculation zone (left) and at an elevation in the flow developed region (right).
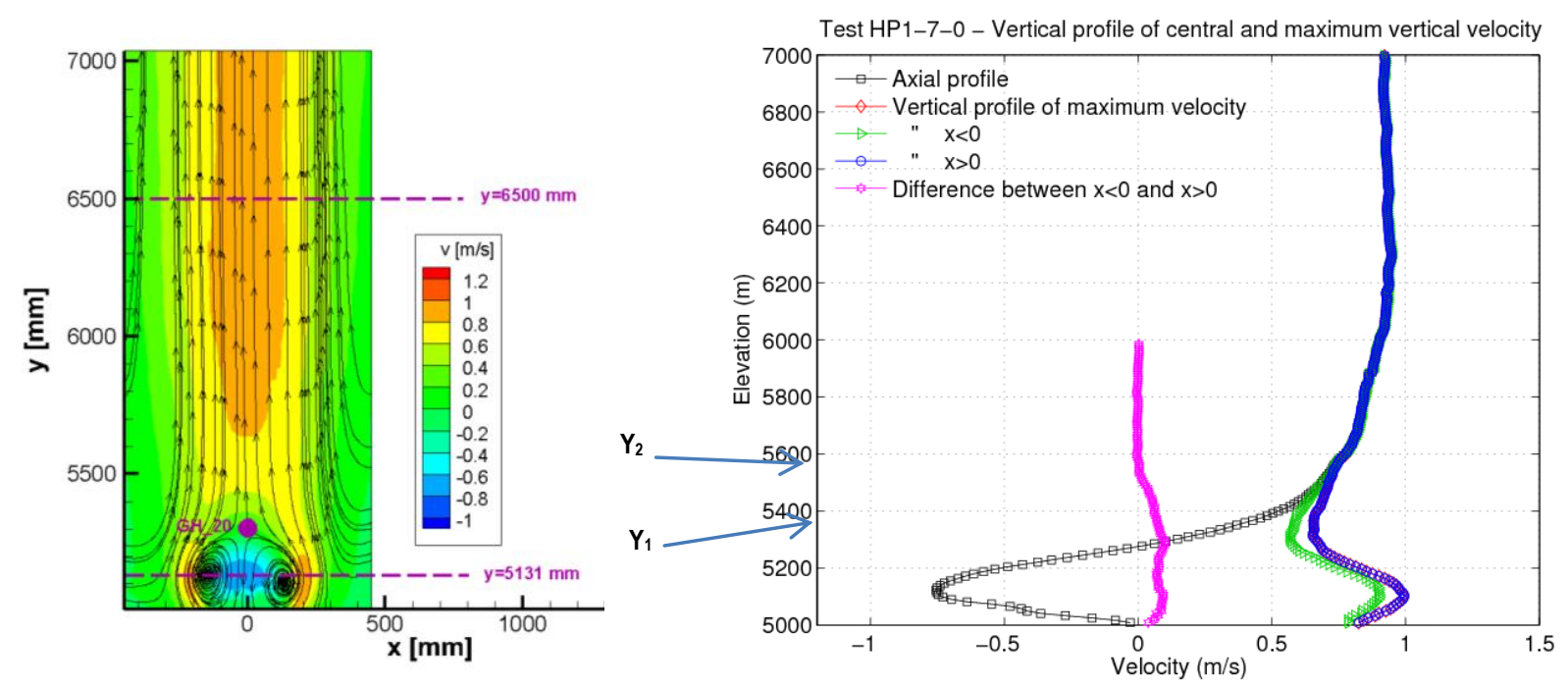

Figure 15: Open benchmark, Step 2: Average experimental flow field (left) measured in Test HP1_7_0, and vertical velocity profiles used in the comparisons with calculated results (right). 

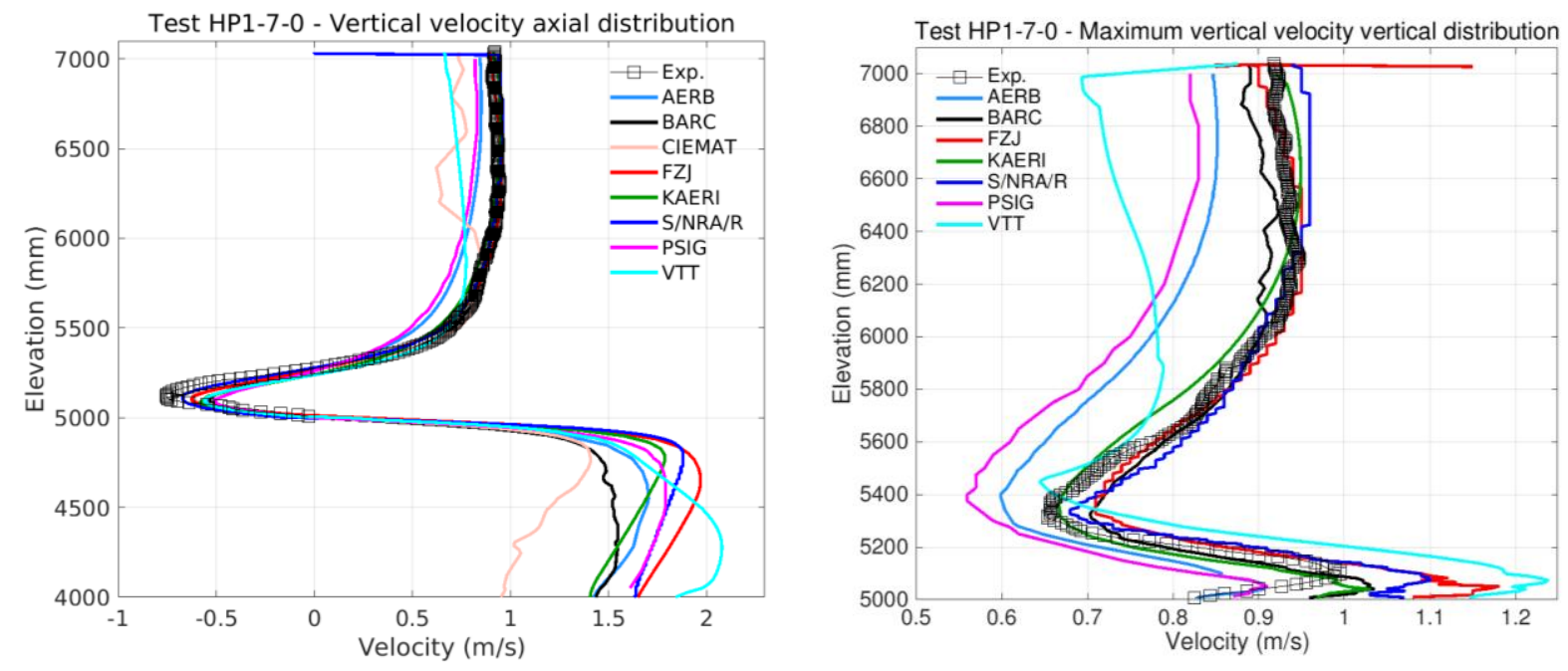

Figure 16: Open benchmark, Step 2: Axial profile of the vertical velocity (left) and vertical profile of the maximum vertical velocity (right).
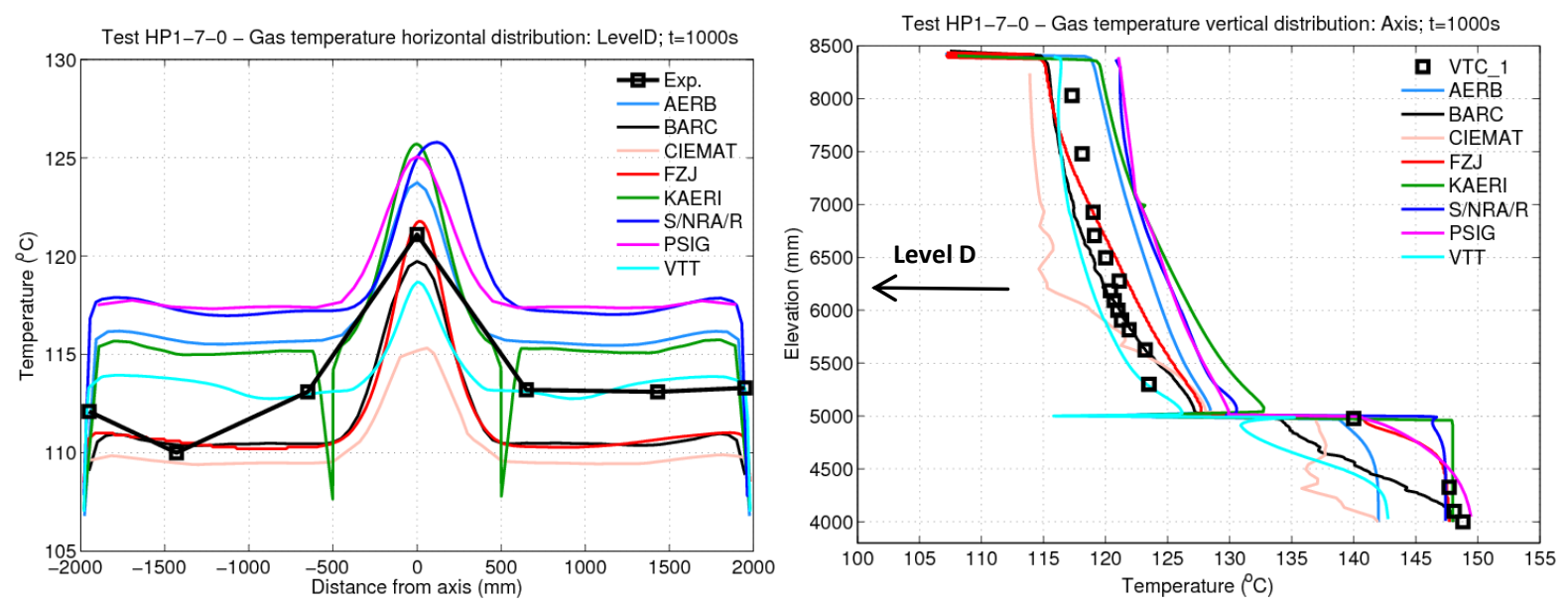

Figure 17: Open benchmark, Step 2: Temperature radial profile at $6.276 \mathrm{~m}$ (left) and axial temperature distribution (right). 

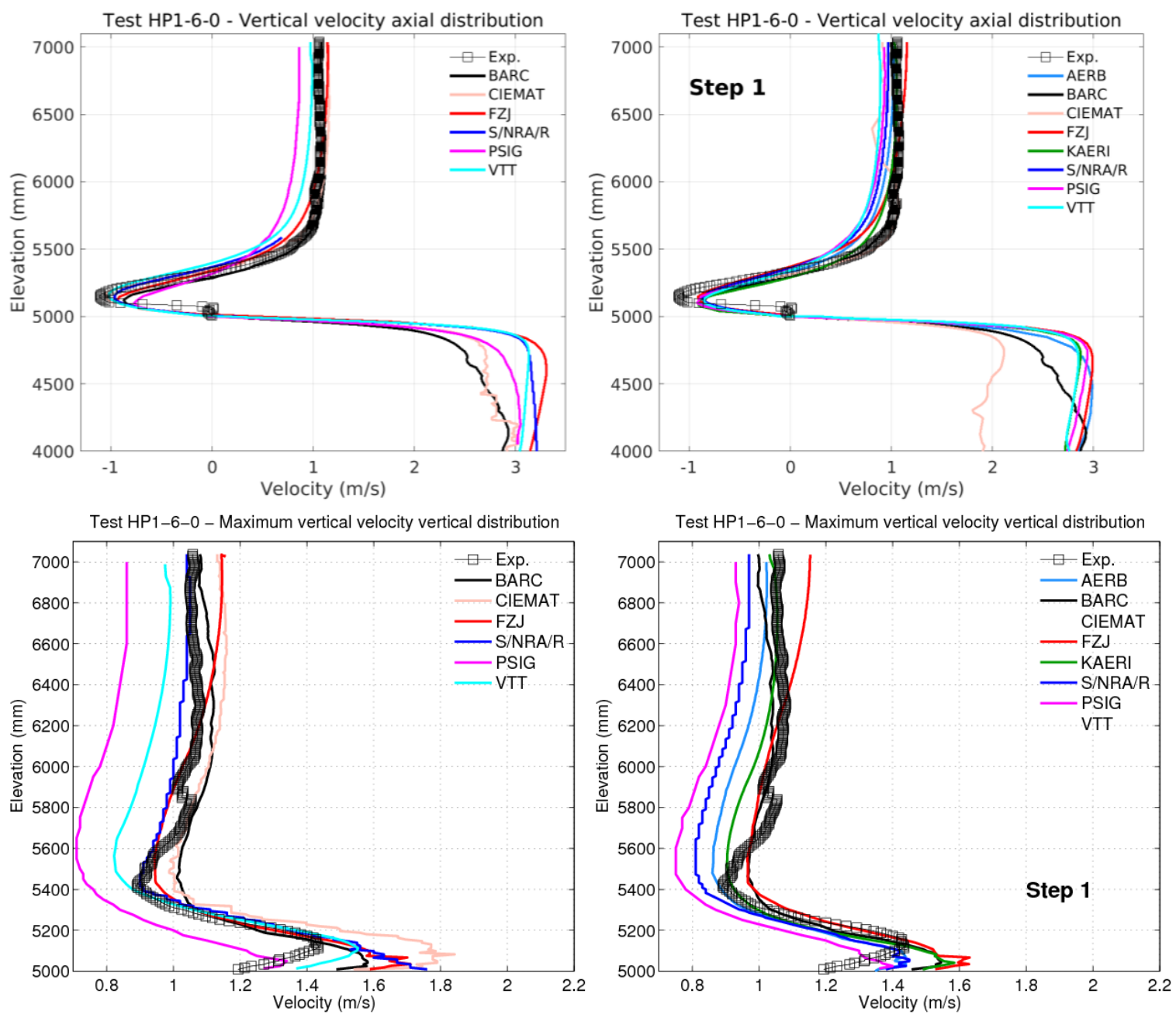

Figure 18: Open benchmark, Step 3: Axial velocity and maximum velocity vertical profiles for Step 3, compared with results for Step 1.
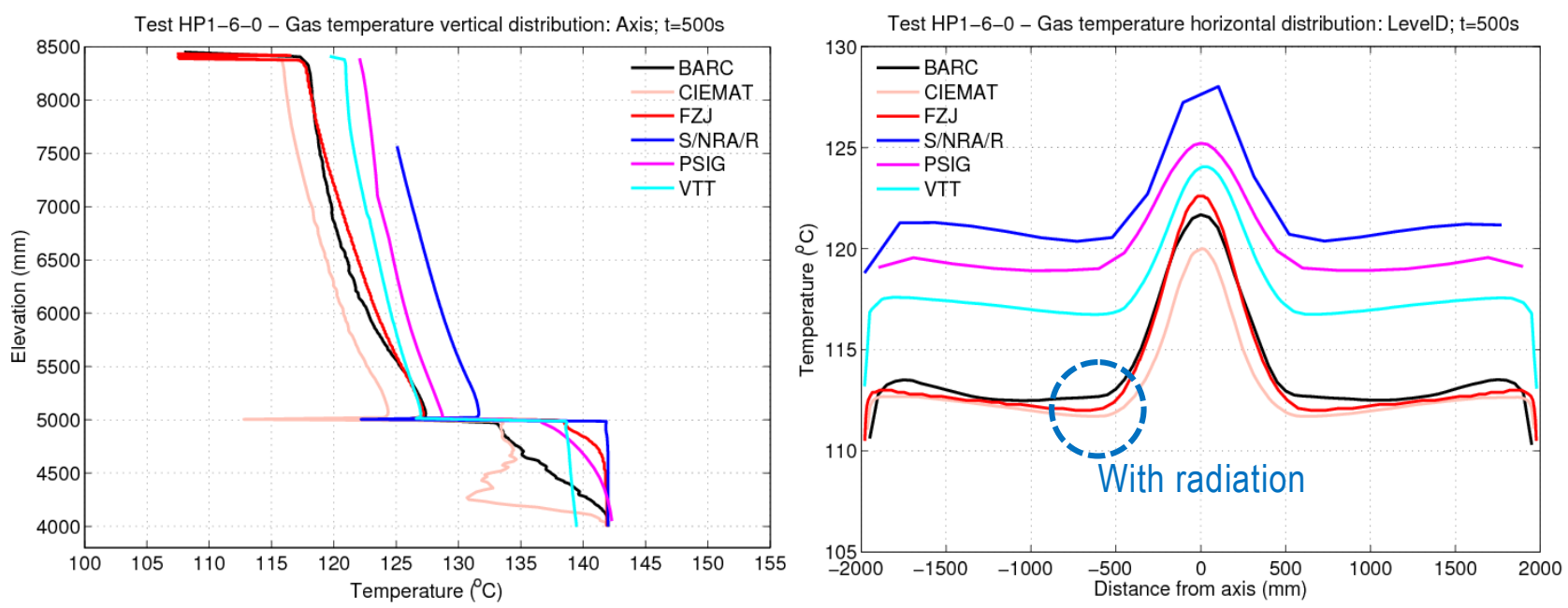

Figure 19: Open benchmark, Step 3: Axial temperature profile and horizontal profile at $6.3 \mathrm{~m}$. 

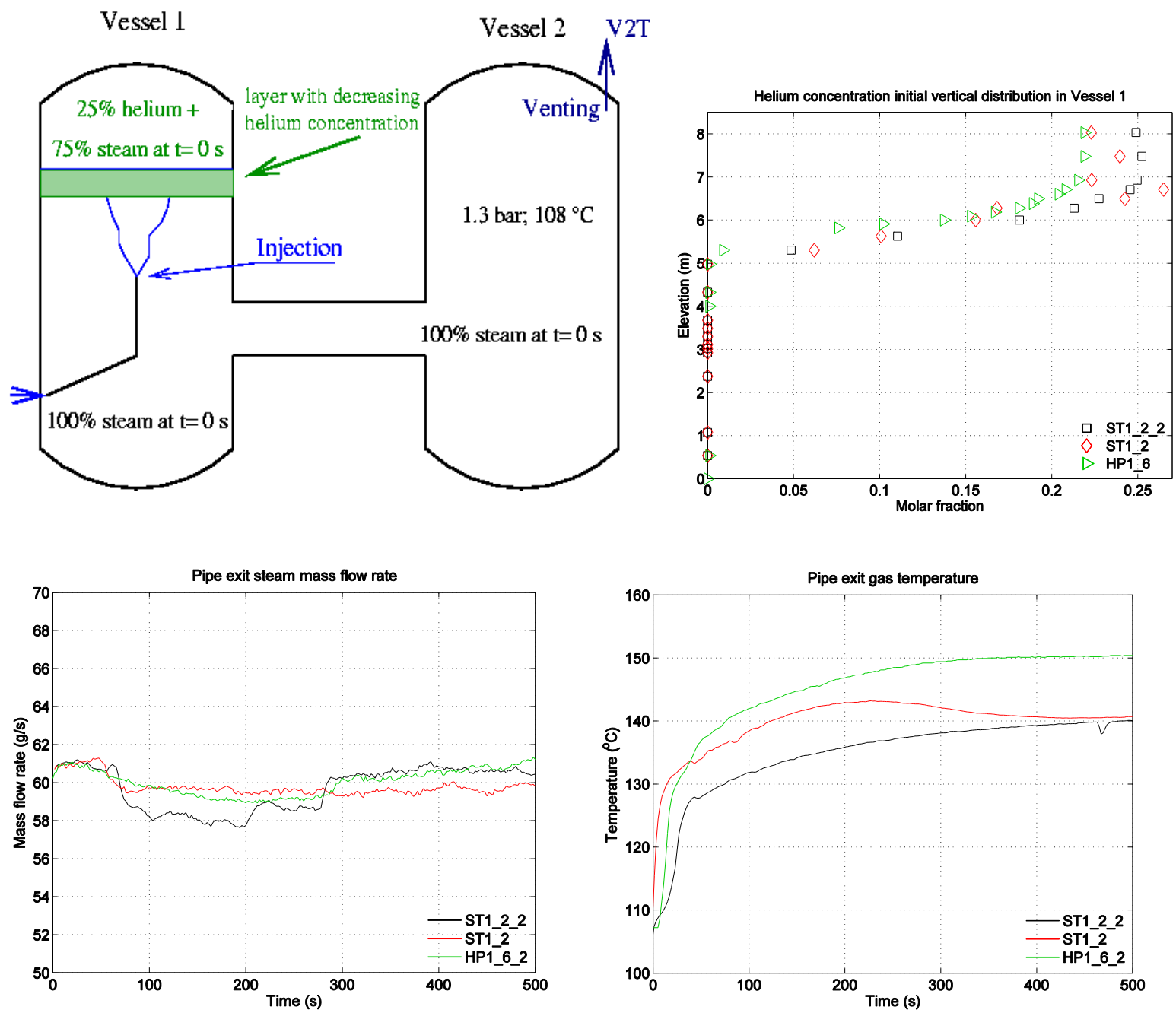

Figure 20: Open benchmark, Step 5: Configuration for SETH-2 test ST1_2_2, and important initial and boundary conditions of test ST1_2 and repetition test ST1_2_2 compared with those used for Test HP1_6_2. 
1

2

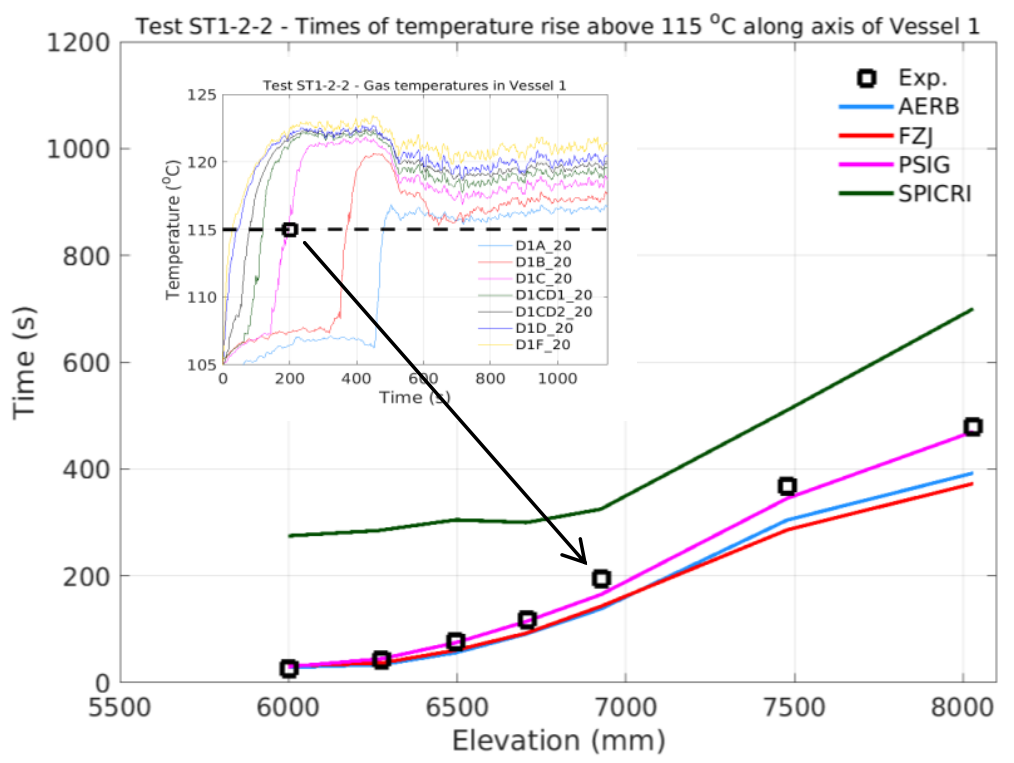

Figure 21: Open benchmark, Step 5: Progression of erosion along the axis in test ST1_2_2, using gas temperature rise times. 

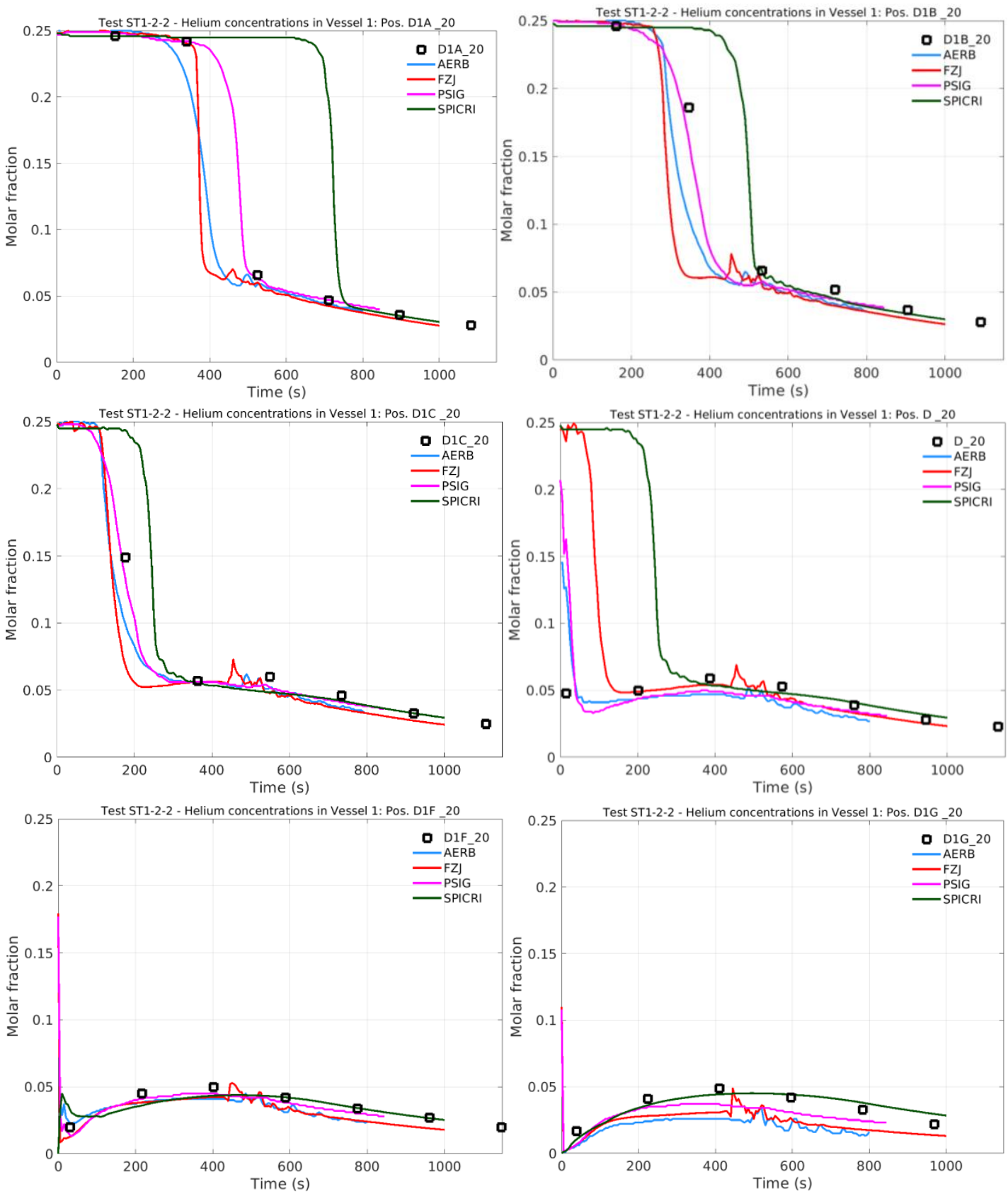

Figure 22: Open benchmark, Step 5: Helium concentration time histories at various elevations along the axis of Vessel 1. 

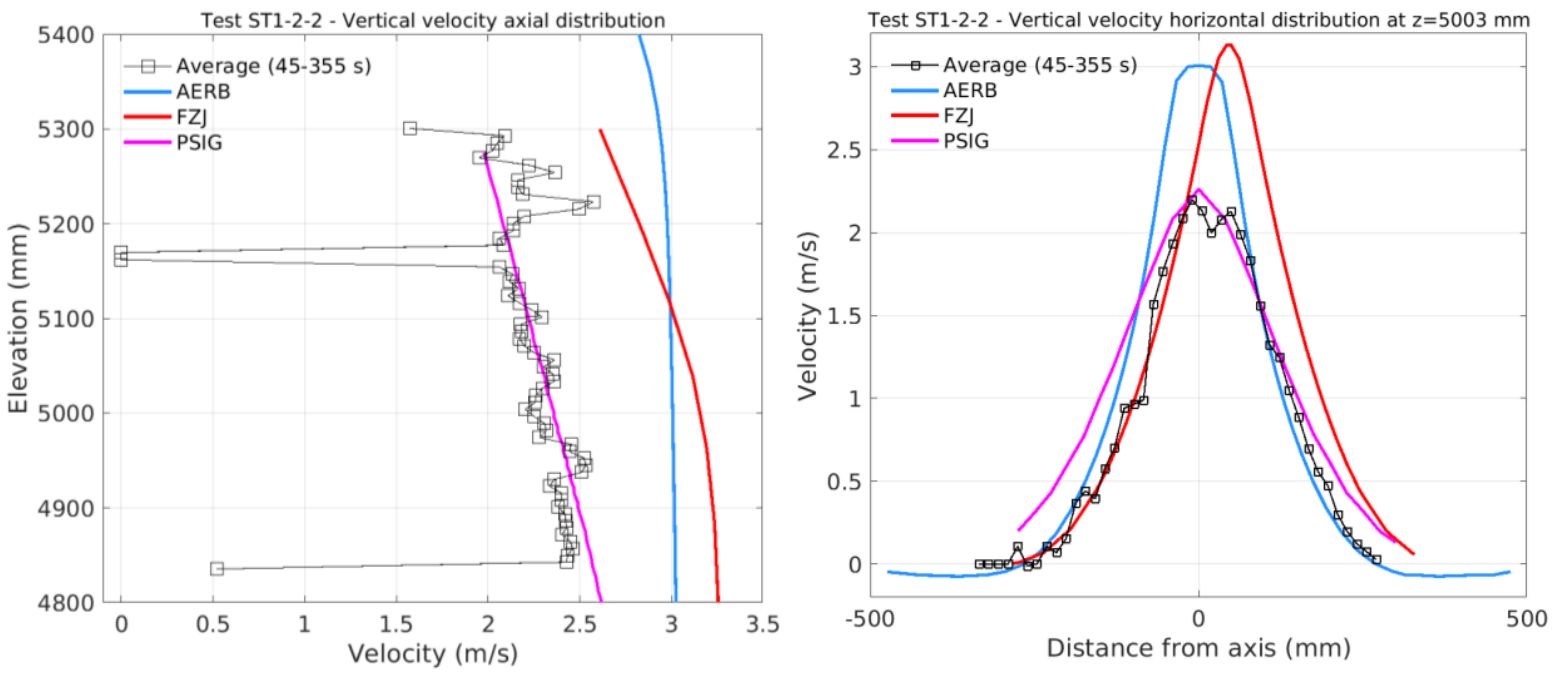

Figure 23: Open benchmark, Step 5: Vertical velocity axial profile (left) and horizontal profile at $\mathrm{z}=5003 \mathrm{~mm}(1 \mathrm{~m}$ above pipe exit)
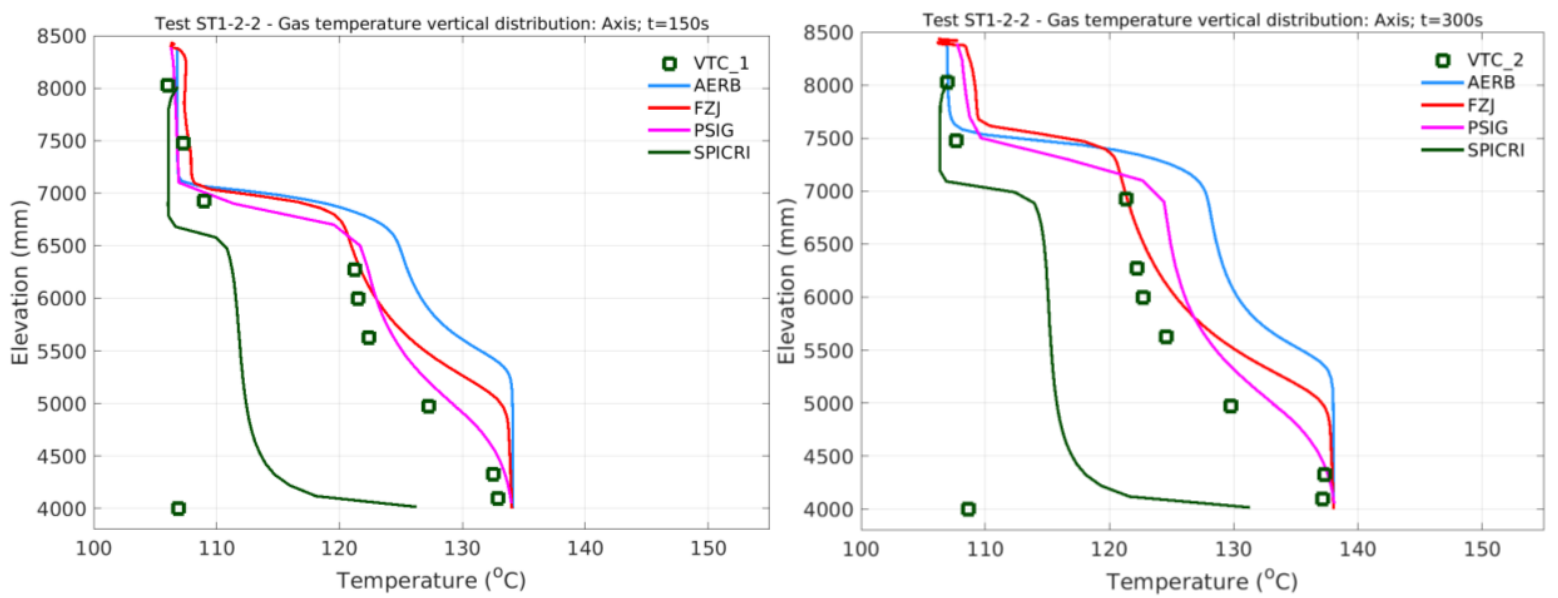

Figure 24: Open benchmark, Step 5: Axial temperature profile at two times.
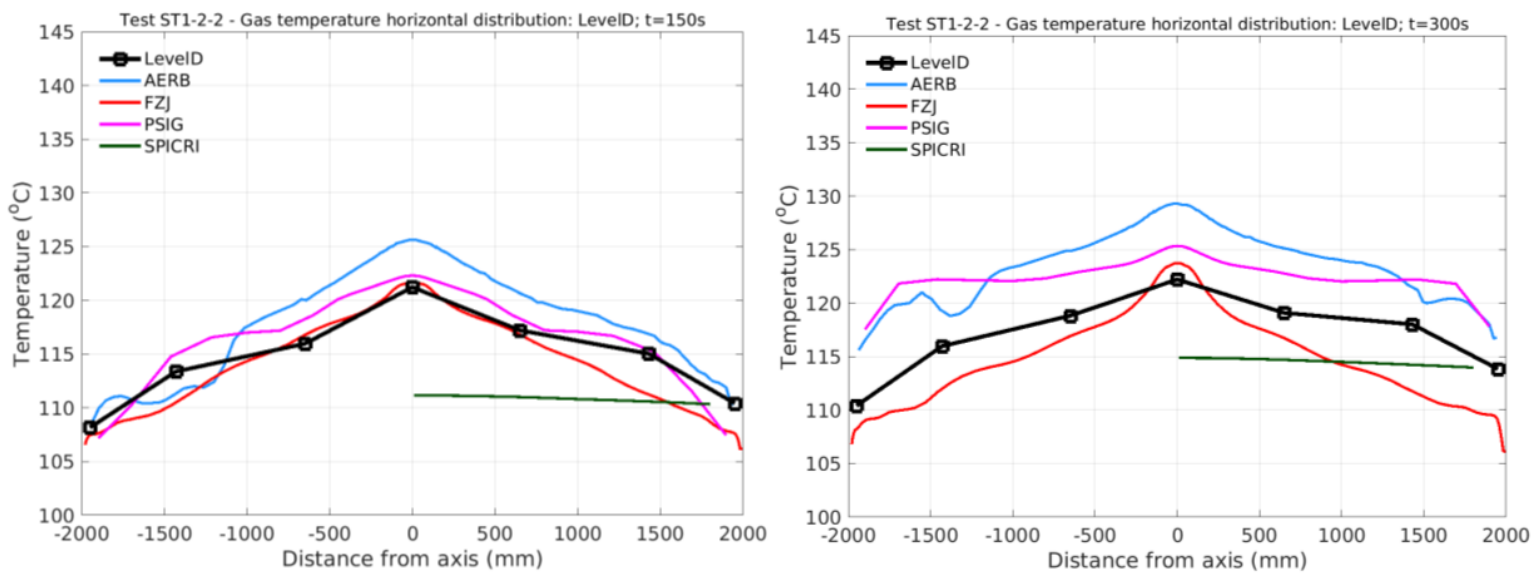

Figure 25: Open benchmark, Step 5: Horizontal profiles at $6.3 \mathrm{~m}$ at $150 \mathrm{~s}$ (before mixing) and $300 \mathrm{~s}$ (after mixing) 

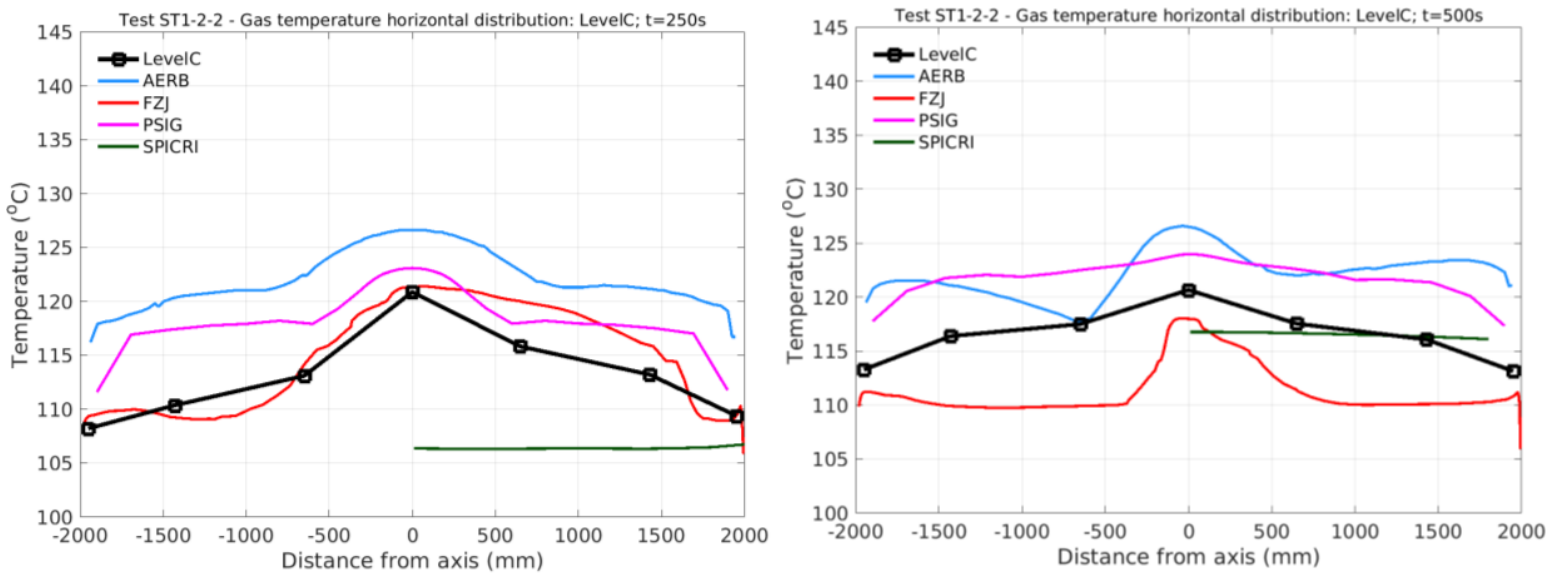

Figure 26: Open benchmark, Step 5: Horizontal profiles at $6.93 \mathrm{~m}$ at $250 \mathrm{~s}$ (during mixing or immediately after) and $500 \mathrm{~s}$ (after mixing)
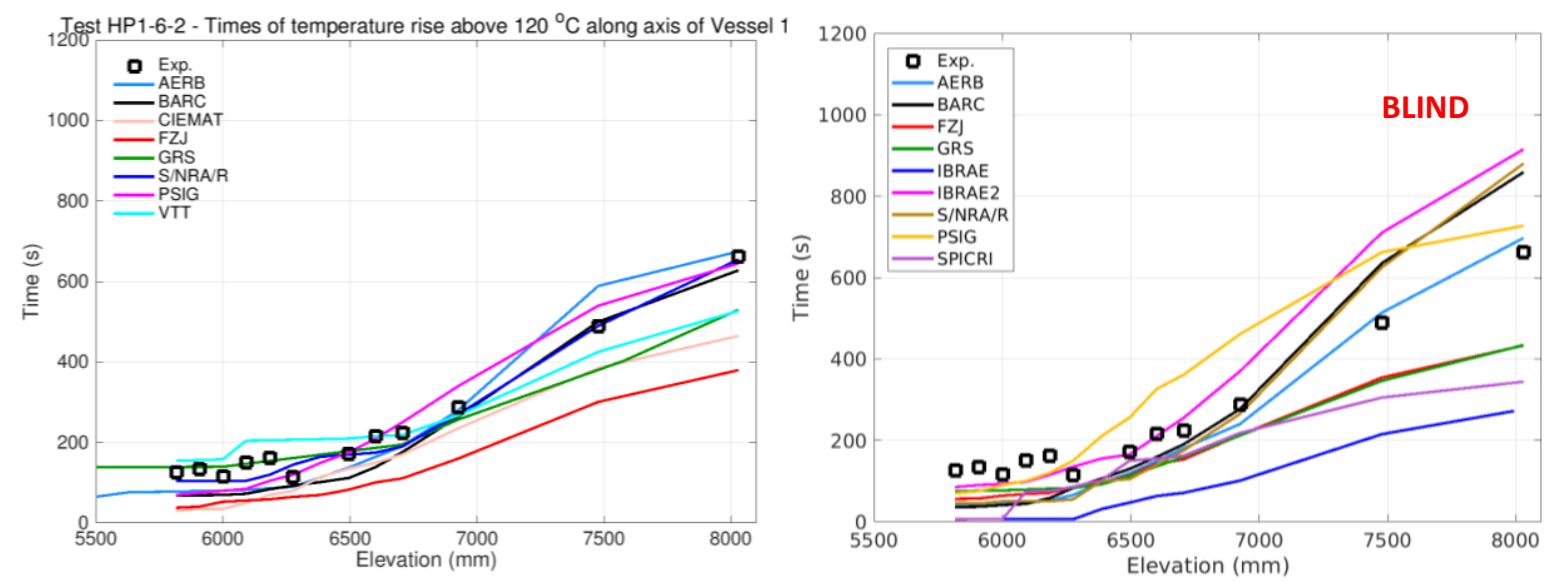

Figure 27: Open benchmark, Step 4: Erosion progression calculated in the open phase (left), compared with best estimate results contributed to the blind benchmark.
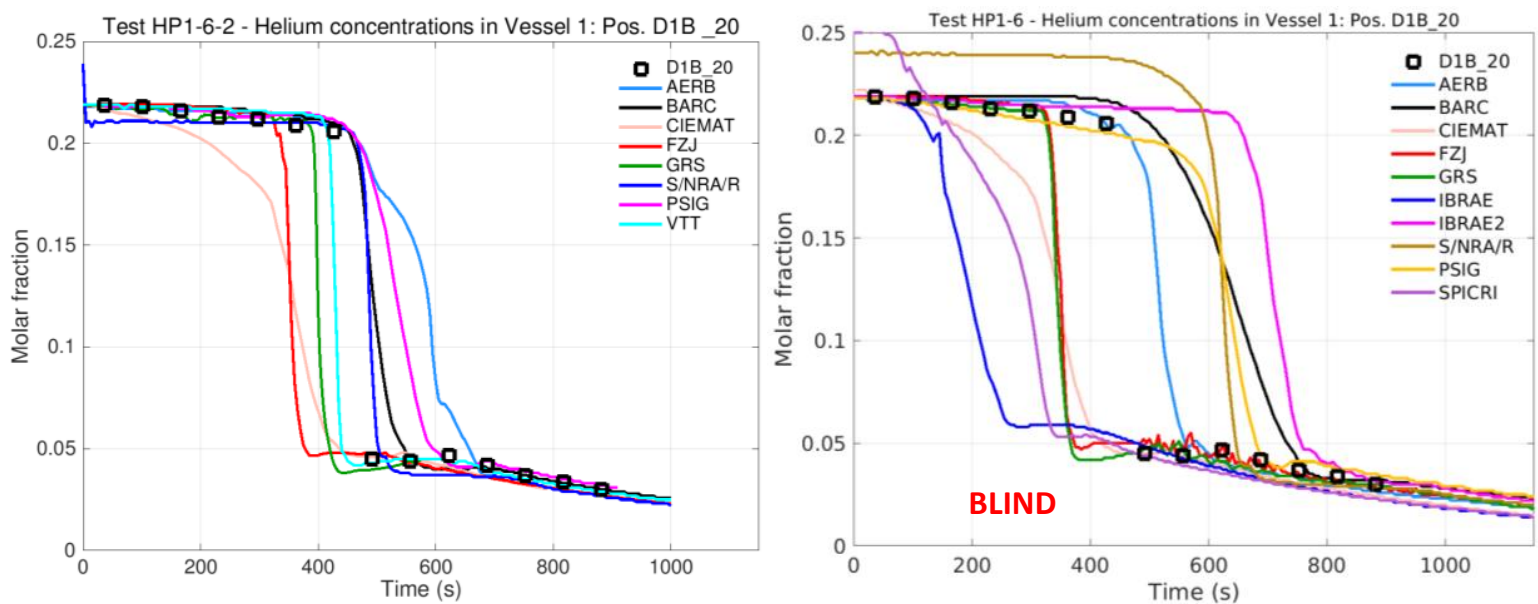

Figure 28: Open benchmark, Step 4: Helium concentration time history at $\mathrm{z}=6.48 \mathrm{~m}$ calculated in the open phase (left), compared with best estimate results contributed to the blind benchmark. 

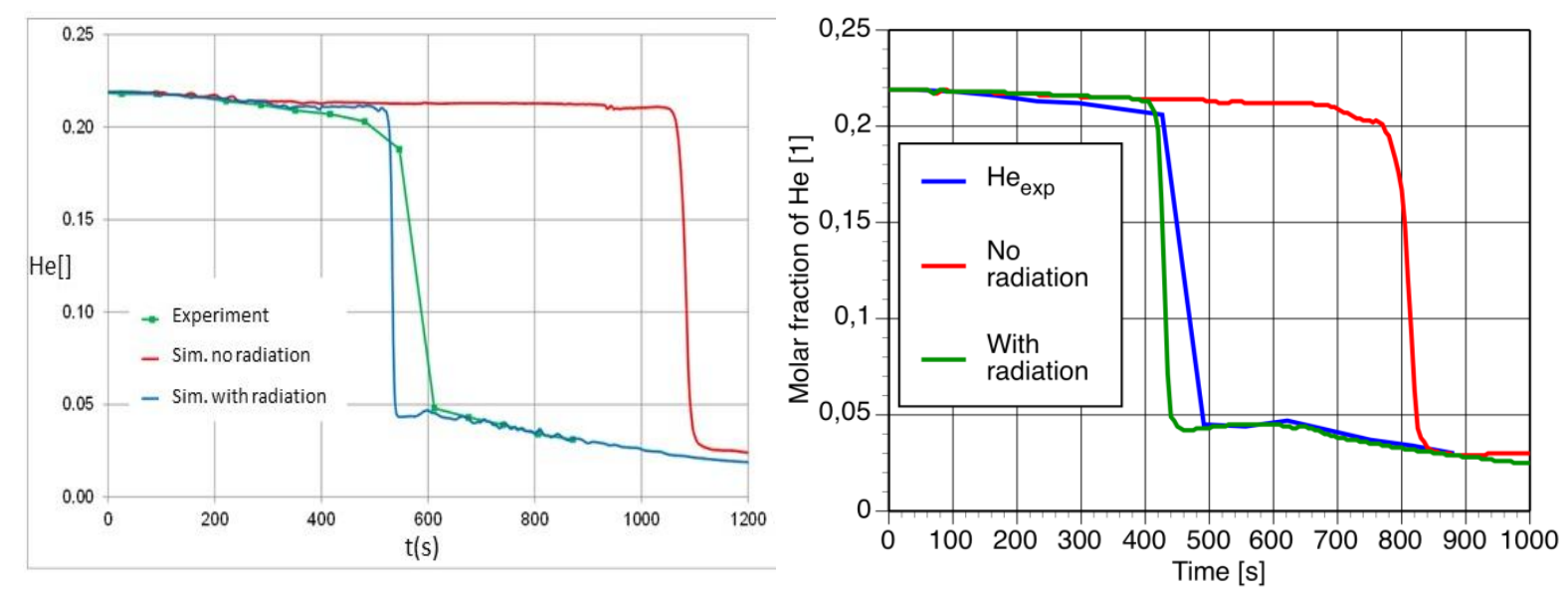

Figure 29: Open benchmark, Step 4: Example of results (helium concentration time histories) obtained at $8 \mathrm{~m}$ (left) by GRS (Schramm et al., 2017) and at $7.5 \mathrm{~m}$ (right) by VTT (Huhtanen, 2018) using the model for radiation or neglecting it.
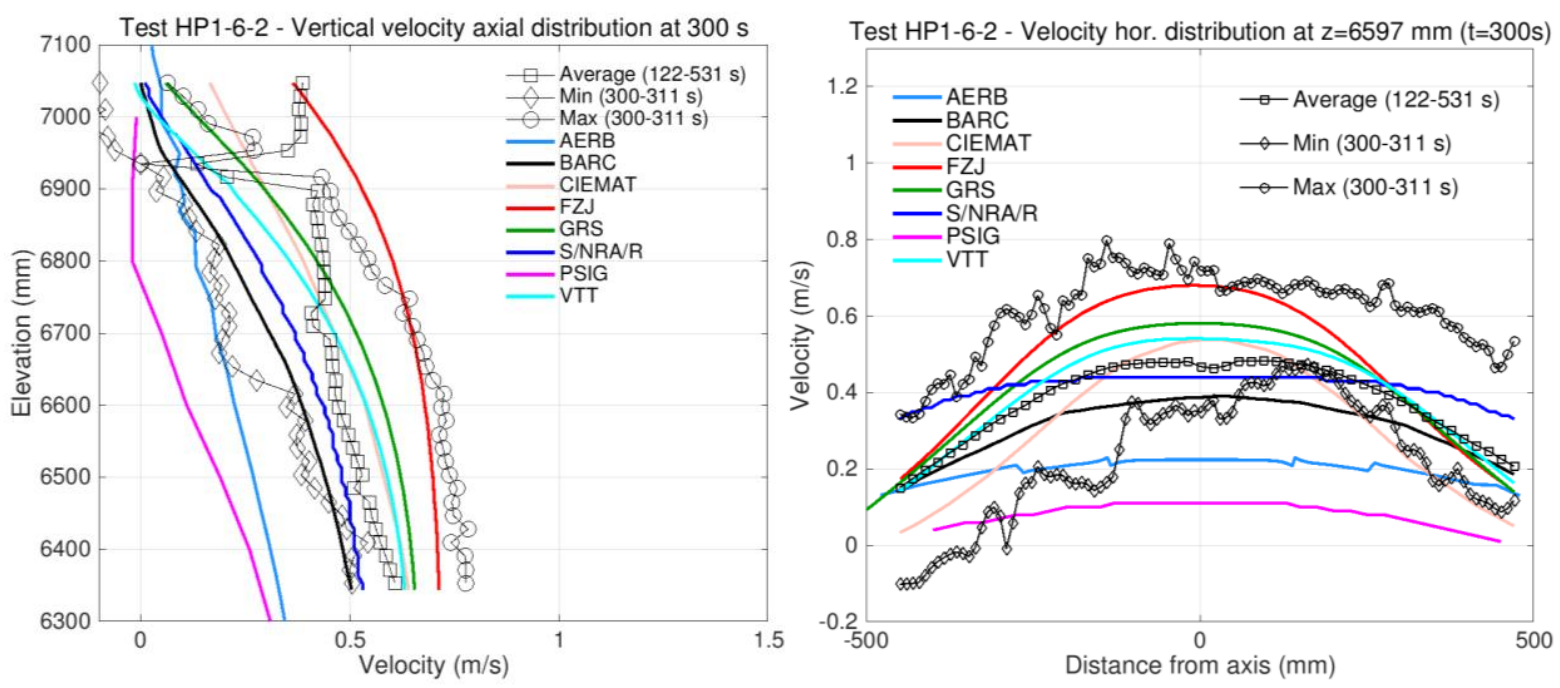

Figure 30: Open benchmark, Step 4: Vertical velocity averaged axial and horizontal profiles in the PIV window. 

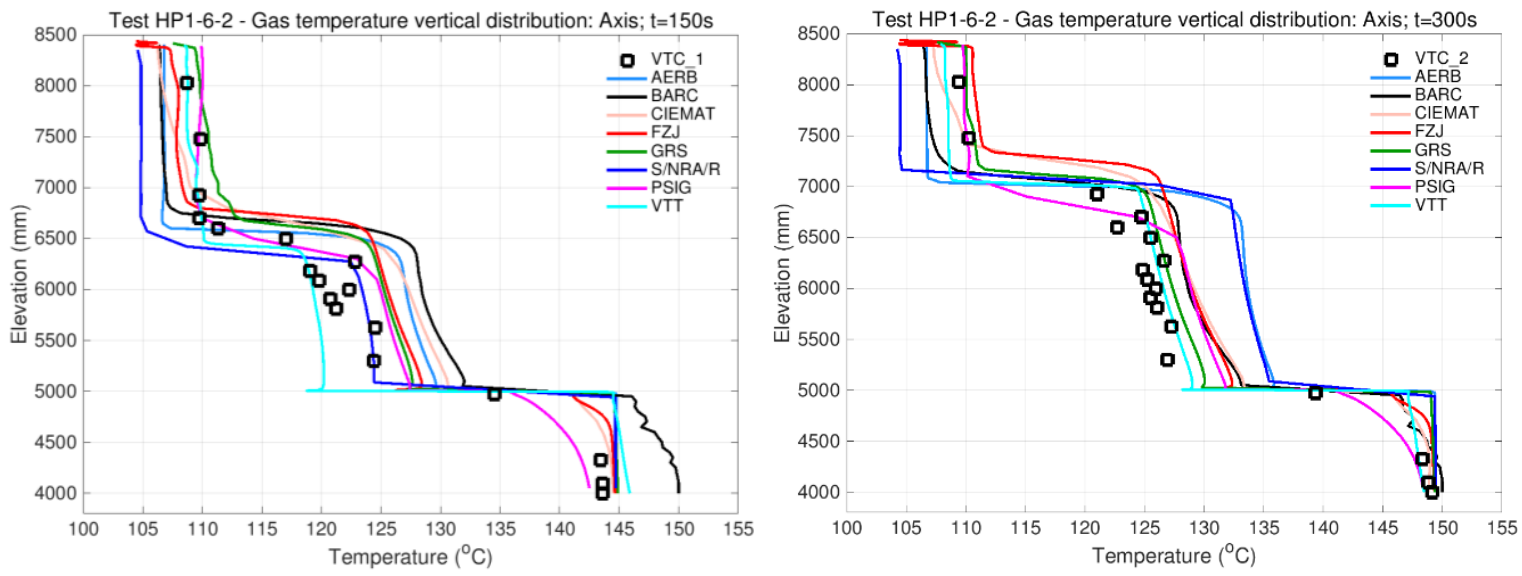

Figure 31: Open benchmark, Step 4: Axial gas temperature distributions at two times. 

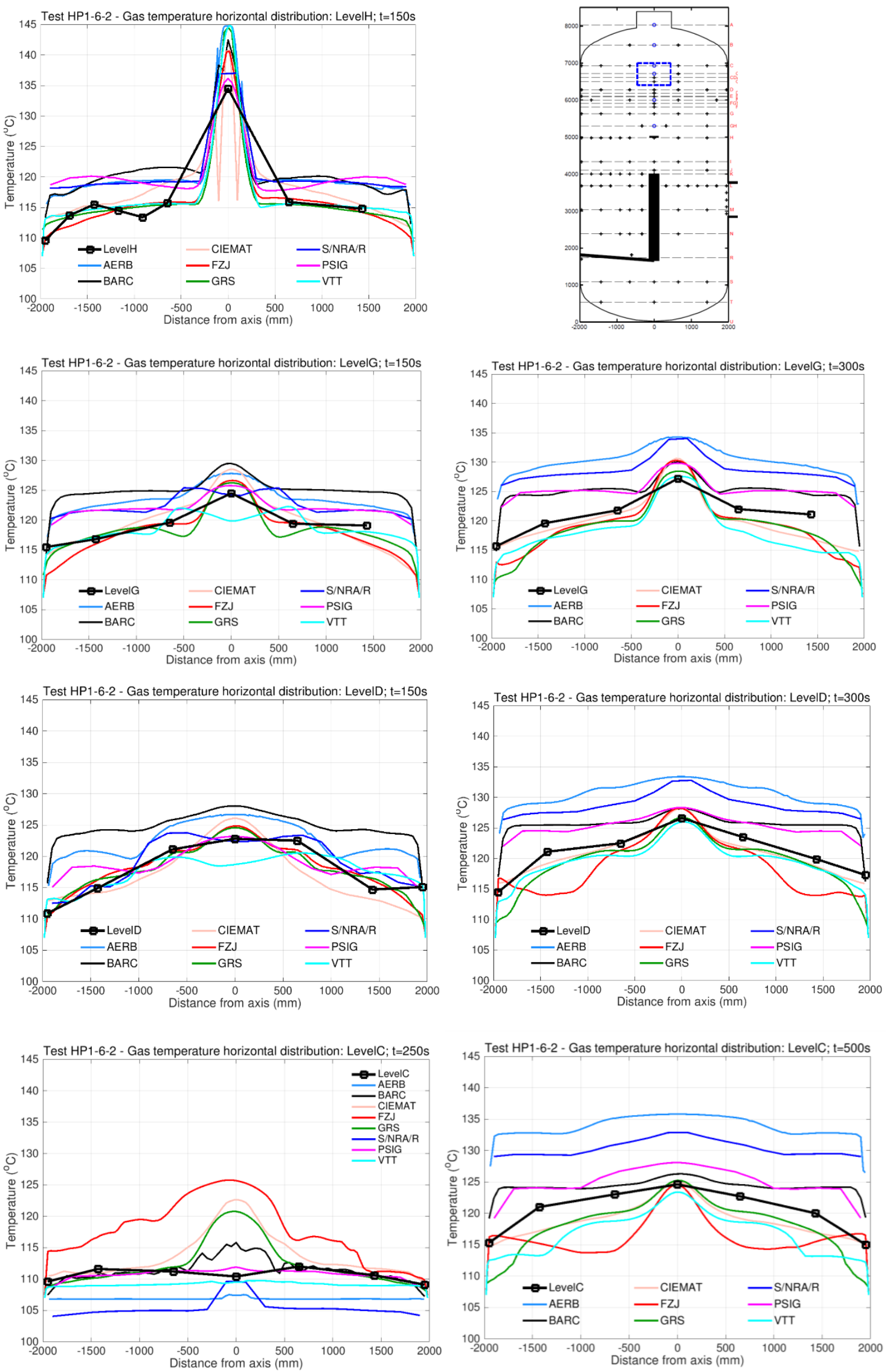

Figure 32: Open benchmark, Step 4: Gas temperature horizontal profiles at various elevations and two times. 

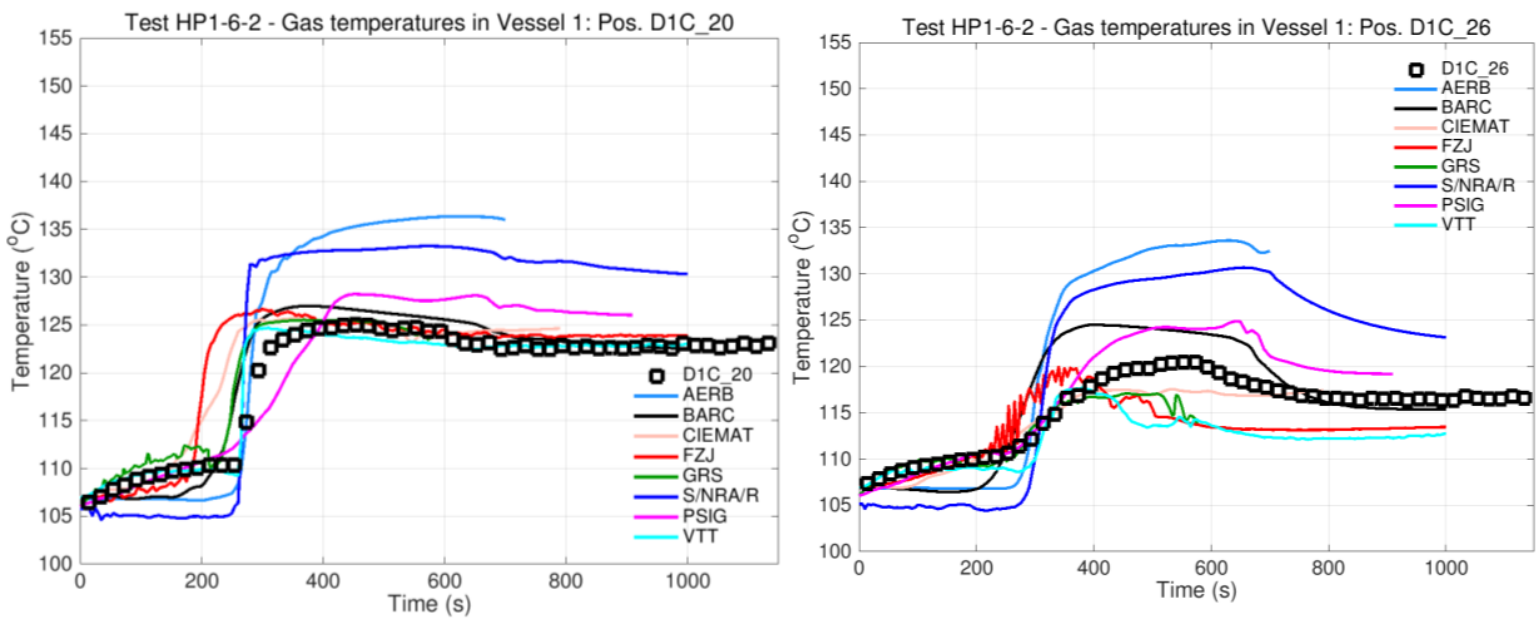

Figure 33: Open benchmark, Step 4: Gas temperature time histories at two positions (left: on the axis; right: at $570 \mathrm{~mm}$ from the wall) at $6.5 \mathrm{~m}$.
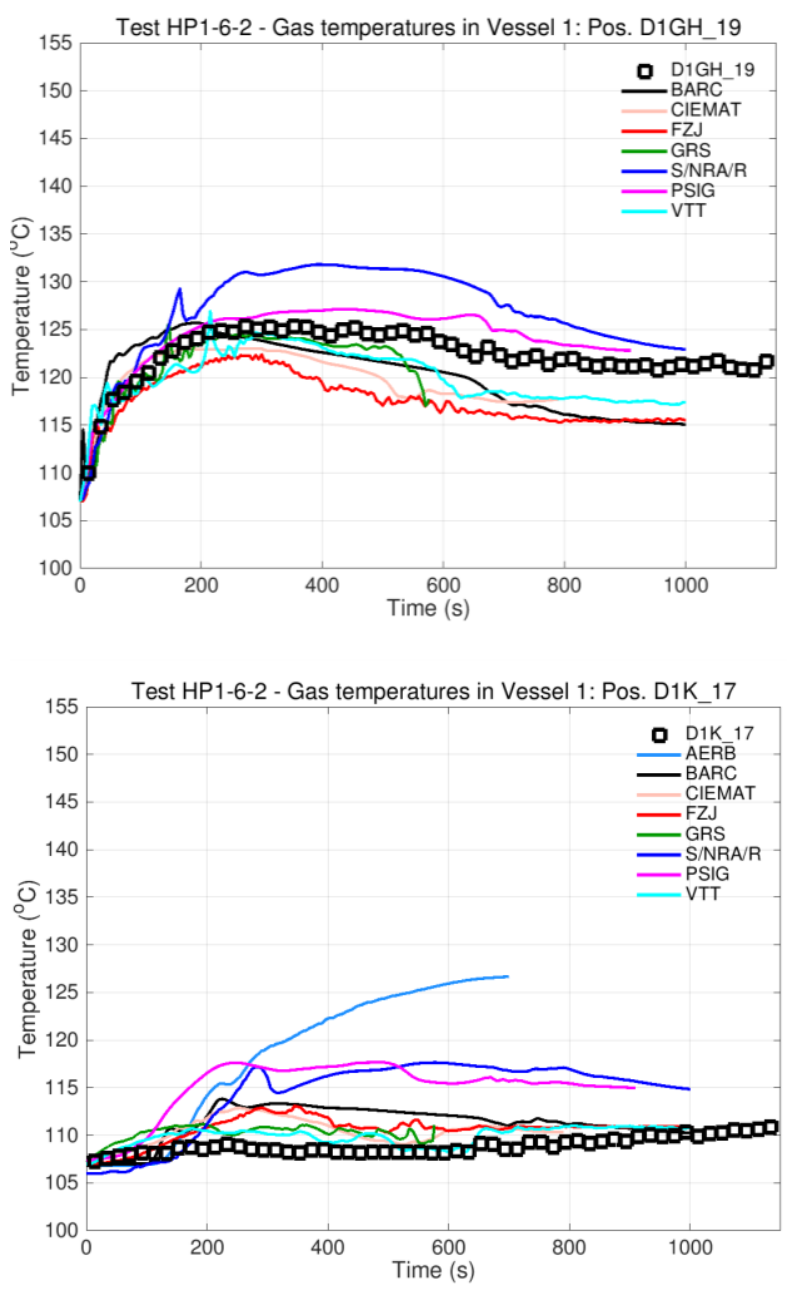

Figure 34: Open benchmark, Step 4: Off-axis $(\mathrm{r}: \pm 325 \mathrm{~mm})$ gas temperature time histories at two positons below the initial bottom of the helium layer (z: 5301 and $4000 \mathrm{~mm}$, respectively) 


\section{REFERENCES}

Abe, S., Studer, E., Ishigaki, M., Sibamoto, Y., and Yonomoto, T. (2018) "Stratification Breakup by a Diffuse Buoyant Jet: The MISTRA HM1-1 and 1-1bis Experiments and their CFD Analysis", Nucl. Eng. Design, 331, pp. 162-175.

Abu-Romia, M.M. and Tien, C.L (1967) “Appropriate Mean Absorption Coefficients for Infrared Radiation of Gases”, Journal of Heat Transfer, 89(4), 321-327.

Allelein, H.-J., Fischer, K., Vendel, J., Malet, J., Studer, E., Schwarz, S., Houkema, M., H. Paillère, H., Bentaib, A. (2007). International Standard Problem ISP-47 on Containment Thermal Hydraulics. Nuclear Energy Agency, Committee on the Safety of Nuclear Installations, Final Report NEA/CSNI/R(2007)10, 2007.

Allein, H.-J., Reinecke, E.-A., Belt, A., Broxtermann, P., and Kelm, S. (2012) “Combined Analytical and Experimental Investigations for LWR Containment Phenomena”, Nucl. Eng, Technol., 44(3), 249-260.

Andreani, M., Badillo, A., and Kapulla, R. (2016a) "Synthesis of the OECD/NEA-PSI CFD Benchmark Exercise”, Nucl. Eng. Design, 299, 59-80

Andreani, M., Daqiang, Y., Gaikwad, A.J., Ganju, S., Gera., B., Grigoryev, S., Herranz, L.E., Huhtanen, R., Kanaev, A., Kelm, S., Kim, J., Nishimura, T., Schramm, B., Sharabi, M., and Paladino, D. (2016b) "Synthesis of a blind CFD benchmark exercise based on a test in the PANDA facility addressing the stratification erosion by a vertical jet in presence of a flow obstruction", OECD/NEA 6th Workshop on Computational Fluid Dynamics for Nuclear Reactor Safety (CFD4NRS-6), MIT, Cambridge, MA-USA, 13-15 September, 2016.

Andreani, M. and Paladino D., (Benchmark Report, 2018), prepared by PSI (with appendices contributed by AERB, FZJ and VTT) (2018) “OECD/NEA HYMERES project: synthesis of results of the benchmark on PANDA test HP1_6_2”, PSI Technical Note AN-42-17-07 Rev.1, Project report HYMERES-P-17-48, May 2018. 
Bentaib, A., Meynet, N., and Bleyer, A. (2015) "Overview on Hydrogen Risk Research and Development Activities: Methodology and Open Issues”, Nucl. Eng. Technol., 47, 26-32.

Breitung, W. and Royl, P. (2000) "Procedure and Tools for Deterministic Analysis and Control of Hydrogen Behavior in Severe Accidents". Nucl. Eng. Design, 202, 249-268.

Chan, C.K. and Jones, S.C. (1997) "Gas Mixing Experiments in a Large Enclosure", Proc. of the $18^{\text {th }}$ Annual Conf. of the Canadian Nuclear Society

Choi, Y.-S., Lee, U.-J., and Park, G.-C. (2001) "Study on local hydrogen behaviors in a subcompartment of the NPP containment", Nucl. Eng, Design, 208, 99-116.

Deri, E., Cariteau, B., and Abdo D. (2010) "Air fountains in the erosion of gaseous stratifications", Int. J. Heat and Fluid Flow, Volume 31, Issue 5, Pages 935-941.

Erkan, N., Mignot, G., Kapulla, R., Paladino, D., Zboray, R., Strassberger, H.J., Bissels, W. and Fehlmann, M. (2009) “OECD SETH-2 PANDA Test ST1_2_2 Quick-Look Report”, PSI internal technical report TM-42-09-07-0, April 2009.

Filippov, A.S., Grigoryev, S.Yu., O.V. Tarasov, O.V. (2017) "On the possible role of thermal radiation in containment thermal-hydraulics experiments by the example of CFD analysis of TOSQAN T114 air-He test”, Nucl. Eng. Design, 310, 175-186.

Gupta, S. (2015) "Experimental Investigations Relevant for Hydrogen and Fission Product Issues Raised by the Fukushima Accident”, Nucl. Eng. Technol., 47, 11-25.

Howell. J.R. (1988) “Thermal Radiation in Participating Media: The Past the Present, and Some Possible Futures", Journal Heat Transfer, 110, 1220-1229.

Huhtanen, R. (2018), Private Communication.

Kapulla, R., Mignot, G., Paranjape, S., Suter, S., Fehlmann, M., and Paladino, D. (2015a) “OECD/NEA HYMERES Project: Jet/Plume interacting with flow obstruction HP1 Series. Test Series Report", PSI internal report TM-42-15-16, Rev.0, Project report HYMERES-P-15-26, Nov. 2015 (report restricted to project participants). 
Kapulla, R., Paranjape, S., Mignot, G., Suter, S., Fehlmann, M., and Paladino D. (2015b) “OECD/NEA HYMERES Project: PANDA Tests HP1_6_0, HP1_7_0, HP1_8_0, and HP1_678_Disk Data Report”, PSI internal report TM-42-15-13 Rev. 0, Project report HYMERES-P-15-25, October 2015 (report restricted to project participants).

Karwat, H. et al. (1999) "State-of-the-Art Report on Containment Thermal-hydraulics and Hydrogen Distribution”, OECD/NEA group of experts, CSNI/R(99)-16 (1999).

Kelm, S., Ritterath, M., Prasser, H.-M., and Allelein, H.J. (2016a) "Application of the MINIPANDA Test Case 'Erosion of a Stratified Layer by a Vertical Jet' for CFD Validation”, Nucl. Eng. Design, 299, 124-135.

Kelm, S., Müller, H., and Allelein, H.-J. (2106b) "Importance of thermal radiation heat transfer modeling in containment typical flows", Paper submitted for presentation at the CFD4NRS-6, MIT, Cambridge, MA, USA, September 13-15.

Liang, R. et al., (2015) "Status Report on Hydrogen Management and Related Computer Codes", NEA/CSNI/R(2014)8,

Lopez-Alonso E., Papini D., and Jimenez G. (2017) "Hydrogen Distribution and Passive Autocatalytic Recombiner (PAR) Mitigation in a PWR-KWU Containment Type", Annals of Nuclear Energy, 109, pp. 600-611.

Mahaffy, J., et al. (2015). Best Practice Guidelines for the Use of CFD in Nuclear Reactor Safety Applications - Revision” NEA/CSNI/R(2014)11.

Noutsopoulos, G.C. and Yannopoulos, P.C. (1989) “Axial Dilution in Obtructed Round Buoyant Jet”, Journal of Hydraulic Engineering, 115(1), pp. 71-81.

Nishimura, T., Hoshi, H. and Hotta, A. (2015) "Current Research and Development Activities on Fission Products and Hydrogen Risk after the Accident at Fukushima Daiichi Nuclear Power Station", Nucl. Eng. Technol., 47, 1-10

OECD/NEA HYMERES-2 project (2017-2021). 
OECD/NEA THAI Project (2010) "Hydrogen and Fission Product Issues Relevant for Containment

Safety Assessment under Severe Accident Conditions" Final Report, 22 June 2010, Report NEA/CSNI/R(2010)3.

Paladino, D. and Dreier, J. (2012) "PANDA a Multi Purposes Integral Test Facility", Science and Technology of Nuclear Installations, 2012, Article ID 239319, doi:10.1155/2012/239319.

Paladino, D., Mignot, G., Kapulla, R., Zboray, R., Andreani, M., Tkatschenko, I., Studer, E., and Brinster, J. (2013) “OECD/SETH-2 Project: PANDA and MISTRA Experiments addressing Key Safety Issues for Water Reactor Containment”, Proceedings of the $15^{\text {th }}$ International Topical Meeting on Nuclear Reactor Thermal-hydraulics (NURETH-15), paper 106, Pisa, Italy, $12-17$ May 12-17, 2013.

Paranjape, S., Kapulla, R., Mignot, G. and Paladino D. (2018) "Gas Redistribution Caused by Interacting Heat Sources in the Presence of a Vertical Condenser," $12^{\text {th }}$ International Topical Meeting on Nuclear Reactor Thermal-Hydraulics, Operation and Safety (NUTHOS-12), Qingdao, China, October 14-18, 2018.

Sarikurt, F.S. and Hassan, Y.A. (2017) "Large Eddy Simulations of Erosion of a Stratified Layer by a Buoyant Jet”, Int. J. of Heat and Mass Transfer, Vol. 112, p. 354-365.

Schramm, B., Stewering, J., and Sonnenkalb M. (2017) “Einsatz von CFD-Codes für die Simulation von unfalltypischen Phänomenen im Sicherheitseinschluss: Validierung und gezielte Modellerweiterung”, Anschlussbericht RS1526, 2017 GRS-472, ISBN 978-3-946607-55-7.

Schwarz, S., Fischer, K., Bentaib, A., Burkhardt, J., Lee, J.-J., Duspiva, J., Visser, D., Kyttala, J., Royl, P., Kim, J., Kostka, P. and Liang, R. (2011) "Benchmark on Hydrogen Distribution in a Containment based on the OECD-NEA THAI HM-2 Experiment”, Nucl. Technol., 175(3), 594-603.

Smith B.L. (2009) "Identification and Prioritization of Generic Nuclear Safety Problems Requiring CFD Analysis", Proc. 17 ${ }^{\text {th }}$ Int. Conf. on Nuclear Engineering (ICONE-17), Paper 75482, Brussels, Belgium, July12-16, 2009. 
Studer, E., Brinster, J., Tkatschenko, I., Mignot, G., Paladino, D., and Andreani, M. (2012)

"Interaction of a light gas stratified layer with an air jet coming from below: Large scale experiments and scaling issues”, Nucl. Eng. Design, 253, 406-412. 


\section{Highlights:}

- A benchmark on gas stratification erosion was conducted using an experiment in the PANDA facility

- A large spread of results was observed in the blind calculations.

- The open phase was multi-step, using complementary tests with enhanced information on velocity field

- Progress was achieved, but several contributions still exhibited large discrepancies

- Results are strongly affected by the modeling of radiation heat transfer from and within the steam 


\title{
SYNTHESIS OF A CFD BENCHMARK EXERCISE BASED ON A TEST IN THE PANDA FACILITY ADDRESSING THE STRATIFICATION EROSION BY A VERTICAL JET IN PRESENCE OF A FLOW OBSTRUCTION
}

\author{
Michele Andreani $^{*(1)}$, Avinash J Gaikwad ${ }^{(2)}$, Sunil Ganju ${ }^{(3)}$, Bhuvaneshwar Gera ${ }^{(3)}$, \\ Sergey Grigoryev ${ }^{(4)}$, Luis Enrique Herranz ${ }^{(5)}$, Risto Huhtanen ${ }^{(6)}$, \\ Vivek Kale $^{(2)}$, Anton Kanaev ${ }^{(4)}$, Ralf Kapulla ${ }^{(1)}$, Stephan Kelm ${ }^{(7)}$, \\ Jongtae Kim $^{(8)}$, Takeshi Nishimura ${ }^{(9)}$, Domenico Paladino ${ }^{(1)}$, \\ Sidharth Paranjape ${ }^{(1)}$, Berthold Schramm $^{(10)}$, Medhat Sharabi ${ }^{(1)}$, \\ Feng Shen ${ }^{(11)}$, Bai Wei ${ }^{(11)}$, Daqiang Yan ${ }^{(11)}$, Rongjin Zhang ${ }^{(11)}$ \\ (1) Paul Scherrer Institut (PSI), Switzerland \\ (2) Atomic Energy Regulatory Board (AERB), India \\ (3) Bhabha Atomic Research Center (BARC), India \\ (4) Nuclear Safety Institute, IBRAE, Russian Federation \\ (5) Centro de Investigaciones Energéticas, Medioambientales y Tecnológicas (CIEMAT), Spain \\ (6) VTT Technical Research Centre of Finland Ltd, Finland \\ (7) Forschungszentrum Jülich (FZJ), Germany \\ (8) Korea Atomic Energy Research Institute (KAERI), Korea \\ (9) Regulatory Standard and Research Department, \\ Secretariat of Nuclear Regulation Authority (S/NRA/R), Japan \\ (10) Gesellschaft für Anlagen- und Reaktorsicherheit (GRS), Germany \\ (11) State Power Investment Corporation Research Institute (SPICRI), People's Republic of China
}

* Corresponding author: Michele Andreani

Laboratory for Scientific Computing and Modelling

Paul Scherrer Institut, Forschungsstrasse 111, 5232 Villigen PSI, Switzerland

Tel.: ++41563102687

Fax: ++4156310 4481

E-Mail: michele.andreani@psi.ch 



\title{
SYNTHESIS OF A CFD BENCHMARK EXERCISE BASED ON A TEST IN THE PANDA FACILITY ADDRESSING THE STRATIFICATION EROSION BY A VERTICAL JET IN PRESENCE OF A FLOW OBSTRUCTION
}

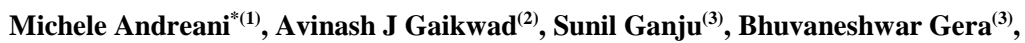 \\ Sergey Grigoryev ${ }^{(4)}$, Luis Enrique Herranz ${ }^{(5)}$, Risto Huhtanen ${ }^{(6)}$, \\ Vivek Kale ${ }^{(2)}$, Anton Kanaev ${ }^{(4)}$, Ralf Kapulla ${ }^{(1)}$, Stephan Kelm ${ }^{(7)}$, \\ Jongtae Kim $^{(8)}$, Takeshi Nishimura ${ }^{(9)}$, Domenico Paladino ${ }^{(1)}$, \\ Sidharth Paranjape ${ }^{(1)}$,Berthold Schramm ${ }^{(10)}$, Medhat Sharabi ${ }^{(1)}$, \\ Feng Shen ${ }^{(11)}$, Bai Wei ${ }^{(11)}$, Daqiang Yan ${ }^{(11)}$, Rongjin Zhang ${ }^{(11)}$ \\ (1) Paul Scherrer Institut (PSI), Switzerland \\ (2) Atomic Energy Regulatory Board (AERB), India \\ (3) Bhabha Atomic Research Center (BARC), India \\ (4) Nuclear Safety Institute, IBRAE, Russian Federation \\ (5) Centro de Investigaciones Energéticas, Medioambientales y Tecnológicas (CIEMAT), Spain \\ (6) VTT Technical Research Centre of Finland Ltd, Finland \\ (7) Forschungszentrum Jülich (FZJ), Germany \\ (8) Korea Atomic Energy Research Institute (KAERI), Korea \\ (9) Regulatory Standard and Research Department, \\ Secretariat of Nuclear Regulation Authority (S/NRA/R), Japan \\ (10) Gesellschaft für Anlagen- und Reaktorsicherheit (GRS), Germany \\ (11) State Power Investment Corporation Research Institute (SPICRI), People's Republic of China
}

Laboratory for Scientific Computing and Modelling

Paul Scherrer Institut, Forschungsstrasse 111, 5232 Villigen PSI, Switzerland

Tel.: ++41563102687

Fax: ++41563104481

E-Mail: michele.andreani@psi.ch 


\begin{abstract}
The benchmark exercise discussed in this paper was conducted within the OECD/NEA project HYMERES. The specific experiment in the PANDA facility chosen for the present benchmark addresses the stratification erosion in a vessel where the upper region contained initially a mixture of steam and helium, and the remaining volume was filled with steam. The mixing is induced by a vertical steam jet, which originates from the exit of a circular pipe located below the bottom of the helium-rich layer. The-stratification erosion process mixing is somewhat slowed down by a small circular plate above the jet source. The exercise consisted of a blind phase, and an open phase. Two sets of blind simulations were requested: one set obtained using a "common model", and a second set produced by a "best estimate" model. For the "common model”, a list of recommendations was given, whereas for the "best estimate" model, each participant was free to choose the modelling approach. The submitted results for the erosion times were in a large band, and especially the large differences in the results with the "common model" were not expected. The results of the best estimate simulations showed that the combination of mesh and modelling approach can lead to a wide spread of results. The most important difficulty in interpreting the results and finding the reason of the large deviations was the lack of information on the velocity field downstream of the obstruction. Therefore, for the open phase extended data from auxiliary, "zero" tests (for similar conditions but without helium layer) were provided to the participants to permit a more basic validation of their models, using a "multi-step approach". The step-by-step validation permitted some progress with respect to some of the items identified in the blind benchmark. However, large discrepancies with data in the final analyses of the test are observed, which cannot be easily attributed to specific model deficiencies or insufficient detail of the mesh. These results raised some questions in relation to best practice guidelines for the use of Computational Fluid Dynamic (CFD) codes for containment analysis and indicated needs for further CFD-grade experiments.
\end{abstract}

Keywords: Containment, stratification erosion, buoyant jet, benchmark, PANDA 


\section{Highlights:}

- A benchmark on gas stratification erosion was conducted using an experimenttest in the PANDA facility

- A large spread of results was observed in the blind calculations.

- The open phase was multi-step, using complementary tests with enhanced information on velocity field

- Progress was achieved, but several contributions still exhibited large discrepancies

- Results are strongly affected by the modeling of radiation heat transfer from and within the steam 


\section{INTRODUCTION}

Hydrogen generated during a-(postulated) severe accident with core degradation is a major safety issue (Karwat et al., 1999; Breitung and Royl, 2000; Bentaib et al., 2015; Lopez-Alonso et al., 2017), because deflagration or detonation might challenge the structural integrity of the containment. The concern about hydrogen risk and the demand for additional research on accident scenarios and on mitigation measures grew after the Fukushima Daiichi accident (Liang et al, 2015; Gupta, 2015; Nishimura, et al, 2015). In particular, hydrogen stratification can substantially increase the risk, as this could lead to local pockets of mixtures with high concentration of this flammable gas (Choi et al., 2001). A special concern is thus the build-up and persistence of stratification of hydrogen in certain regions, which has to be addressed by dedicated experimental research and accurate analyses (Smith, 2009). Various experimental programmes (Allelein et al., 2007; Deri et al., 2010; OECD/NEA THAI Project, 2010; Allelein et al., 2012; Studer et al., 2012; Paladino et al., 2013; Kapulla et al., 2014) and code validation activities (Schwarz et al., 2011; Kelm et al., 2016a; Andreani et al., 2016a; Sarikurt and Hassan, 2017; Abe et al., 2018) have included in-depth investigations on stratification formation and break-up/erosion processes. This research included, among others, the investigation on the interaction of a gas plume or jet with a density interface in an open space, typical of the situation in the dome of the containment. However, since the multi-compartment geometry of many containment designs is quite complex, it is necessary to assess the capability of the codes to simulate the effect of various structures on the evolution of the distribution of gases in the presence of flow obstructions. In particular, the effect on mixing of an obstruction at short distance from the origin of the efflux is of general interest (e.g., Noutsopolos and Yannopoulos, 1989), but only a few investigations with light gases exist in open literature (e.g., Chan and Jones, 1997). A first series of experiments in PANDA addressing this issue have been conducted within the HYMERES project.

HYMERES (HYdrogen Mitigation Experiments for REactor Safety) is the acronym for an OECD/NEA project (2013-2016), which is supported by thirteen countries and centered around experiments performed in the PANDA and MISTRA facilities, located at PSI in Switzerland and CEA in France, respectively (Paladino et al., 2012). The project includes various series of experiments (with helium used as simulant for hydrogen), where the mixing of a stratified atmosphere is controlled by jets or energy sinks/sources (such as heaters, coolers, etc.). In PANDA, stratified conditions, with a helium-rich layer at the top of one steam-filled vessel, are either initially prescribed or built during the transient. One of these experiments in PANDA (HP1_6_2) was chosen for the present benchmark, which is similar to the recent OECD/NEA PANDA Benchmark (Andreani et al., 2016a). It addresses again the stratification erosion induced by a vertical jet, but in this new test, with injection of steam instead of air, and with an obstruction above the pipe exit. The conditions of the experiment address an idealised accident scenario, where a dry release of pure light gas (Hydrogen in Light Water 
reactors) is followed by a phase of steam release. In most postulated scenarios, stratification and mixing would be in competition, due to the continuous efflux into the containment and operation of safety features. The simpler conditions investigated in HYMERES are, however, more suitable for fundamental code validation. In other projects (e.g. ERCOSAM-SAMARA, Paladino et al., 2018), stratification break-up caused by the operation of safety measures was investigated, neglecting the continuous steam release.

The OECD /NEA PANDA benchmark indicated that even for simple conditions the successful application of CFD to containment flows is still hindered by the special feature of the typical problems, i.e. long transients in very large fluid domains. Under these conditions, the application of Best Practice Guidelines (Mahaffy et al., 2015) proves to be very demanding, and often nearly impossible with the available project times and computing resources. Benchmarking activities (especially based on blind predictions) are therefore very important to reveal the weaknesses of modelling strategies established on the base of previous experience when a new problem has to be tackled and sensitivity studies must be limited to a few runs.

In order to enhance the interpretation of the comparison of the calculated results with the experimental data, for the blind benchmark the participants were requested to submit two sets of results: one set should be obtained using a "common model", and a second set produced by a "best estimate" model. "The common model" was an attempt to overcome the difficulties to identify merits and limitations of the simulations due to the effect of a large number of parameters, which, for instance, affected the OECD/NEA benchmark (Andreani et al., 2016a). For the "common model", a list of recommendations was given with respect to initial and boundary conditions (e.g., homogeneous initial vertical gas and wall temperatures, modelling of the injection pipe), as well as concerning model selection (no condensation, no radiative heat transfer, standard k- $\varepsilon$ turbulence model). For the "best estimate" model, each participant was free to choose the modelling approach that was considered to be the best suited to the physical problem investigated, also on the base of previous experience, and to use refined representation of initial and boundary conditions. Each participant was expected to submit only one set of results for each of the two models. The participants were encouraged to use Best Practice Guidelines (BPG) to provide the most trustworthy set of results, but only few participants could afford more than a mesh sensitivity study using two meshes.

Both sets of submissions, i.e. those using a "Common Model" (CM) and those using a "Best Estimate Model” (BEM) model produced a large variety of results, leaving open a number of questions. The open phase of the benchmark was then considered necessary to provide more information on the importance of some physical effects and the capability of the various modelling strategies adopted by the participants to address them. 
The present paper reports the outcome of the benchmark. Results for the entire set of variables requested are collected and discussed in Andreani and Paladino (2018), which will be referred to as the Benchmark Report (2018). Here, only the main aspects of the benchmark are discussed. Since the results of the blind phase were already illustrated in Andreani et al. (2016b), the paper focuses on the open phase of the benchmark.

\section{THE EXPERIMENT}

Only the essential information necessary for comparing the test results and the simulations is provided in this chapter. A complete presentation of the tests and of the main experimental results is included in a project report (Kapulla, et al., 2015a).

\subsection{Configuration and test conditions}

The PANDA facility (Paladino and Dreier, 2012) is a multi-compartment, large-scale thermalhydraulics test rig located at the Paul Scherrer Institute (PSI), Switzerland. For these series of tests only the two upper vessels were used (Fig. 1, where the large manholes at the top of the vessels are also visible), which are $8 \mathrm{~m}$ in height and $4 \mathrm{~m}$ in diameter. In Fig. 1, the nominal conditions of the test are indicated. The PANDA vessels and the major internal penetrations/flanges are made of stainless steel. All external surfaces are insulated, and the heat losses have been experimentally determined over a broad range of temperatures. The experiment mainly addresses the evolution of the thermalhydraulic variables in the vessel (injection vessel, on the left in the figure) where the initial stratification was created and the steam was injected. In the injection vessel (Vessel 1), a steam jet originates from a circular pipe located on the axis of the vessel and with an exit located $2 \mathrm{~m}$ below the bottom of the helium-rich layer (which starts at $6 \mathrm{~m}$ ). Steam is produced in the RPV vessel (scaled Reactor Pressure Vessel, used for this function in integral experiments), which, for fundamental tests like those in HYMERES, is configured as the steam source. The mixing is somewhat slowed down by a small circular plate (20 cm in diameter) also centered on the axis and positioned $1 \mathrm{~m}$ above the jet exit. The vessels are kept at approximately constant pressure $(1.3$ bar) during the test by venting the fluid to the atmosphere through a nozzle at the top of Vessel 2.

Prior to the test, saturated steam was injected in the vessels, and the fluid and vessel walls (as well as the obstruction plate) were thus heated to the target temperature, which was set to avoid wall condensation during the transient. Stratified steam/helium conditions then have been created in the test vessel by injecting helium above $6000 \mathrm{~mm}$ (Fig. 1). Just before the beginning of the test, a helium-rich layer occupies the region above the elevation of $5000 \mathrm{~mm}$ (measured from the lowest 
point on the inside of the vessel), the molar fraction of helium increasing non-linearly to about 0.22 at $8000 \mathrm{~mm}$ and above into the manhole space. The region of Vessel 1 below this layer and Vessel 2 are filled with steam. The air concentrations were between 0.1 and $0.2 \%$. The measured helium molar fraction at time $t=0$ as a function of elevation is also displayed in Fig. 1 . The uncertainty in the measurement of gas concentration is $+-1.5 \%$ absolute. All concentration measurements are subject to total combined uncertainties of $<1 \%$. The measured gas and wall temperatures immediately before the start of the transient were between 105 and $108^{\circ} \mathrm{C}$ (nominal value: $108{ }^{\circ} \mathrm{C}$ ). These values ensure that condensation (if any) could not play a role during the transient. All temperature measurements are subject to an uncertainty of $\pm 0.7 \mathrm{~K}$.

The test was started by injecting superheated steam, the flow rate and the temperature being $60 \mathrm{~g} / \mathrm{s}$ and $150{ }^{\circ} \mathrm{C}$, respectively. During the test, gas and wall temperatures at several locations were measured, and helium concentration was measured at 6 elevations above the injection. Moreover, PIV measurements in a zone of fluid initially immersed in the helium-rich layer (far above the plate) provide average velocities and turbulent quantities, and thus some information on the interaction between the jet and the stratified ambient (see below).

\subsection{Main results}

In the experiment, the-time evolutionhistories of gas temperatures and helium concentrations above the injection describe the progression of the erosion process (Fig 2),-The erosionwhich is characterized, at each elevation, by helium concentration drop and temperature increase as a result of the rise of the leading edge of the steam jet and associated mixing (fountain) zone to that height. $\underline{A}$ visualisation of the stratification erosion process in Vessel 1 is provided by the evolution of the twodimensional spatial distributions of gas temperatures in the vertical plane containing the axis (Kapulla et al., 2015a). In section 4.6, some of the calculated results for both gas temperatures and helium concentrations contributed to the open benchmark will be presented.

Since the test was rather fast (within $600 \mathrm{~s}$ the fluid in Vessel 1 was fully mixed), and the scanning rate of the mass spectrometer is necessarily low, the time history of the erosion at the various elevations (which will be used to get a representative measure of the global success of the simulations) was obtained from the temperature measurements (scanning rate $0.5 \mathrm{~Hz}$ ), using a threshold of $120^{\circ} \mathrm{C}$ ( $\mathrm{T}_{\mathrm{lim}}$ in Fig. 2). This procedure for defining the "erosion times" and comparing the measurements with code predictions at the various locations is clearly not accurate, especially at elevations (notably the highest at $8 \mathrm{~m}$ ) where the temperature has a slow and non-uniform increase. Another option would have been to use the times of the inflection points (maximum time derivatives of the temperature curves), but the results would differ from those obtained with the used procedure at 
most by $50 \mathrm{~s}$ (at the highest elevation). The experimental values will be shown together with the results of the simulations in the next section.

Mean velocity measurements were obtained by PIV in a Field of View (FOV) located between the elevations of 6300 and $7000 \mathrm{~mm}$. Measurements and discussion of statistical errors are included in the project report (Kapulla et al., 2015a). Since the averaging period was of $409 \mathrm{~s}$ (centred around $\mathrm{t}_{\mathrm{c}}=$ $326.6 \mathrm{~s}$ ), the use of this information for comparing the simulations with the data at specific times is questionable. The use of the velocity measurements for assessment purposes is reconsidered in the open phase of the benchmark, for which additional data for similar conditions over a larger region above the plate (between 5000 and $7000 \mathrm{~mm}$ ) are made available. Therefore, for the sake of the open benchmark, results for velocities will not be discussed.

\section{RESULTS OF THE SIMULATIONS FOR THE BLIND BENCHMARK}

It was mentioned above that the participants in the benchmark were requested to submit two sets of results, one using the "common model" (CM) and one with the "best estimate model" (BEM). The specifications for the CM (Andreani et al, 2016b) included geometry, treatment of heat transfer at the walls (heat losses, no radiation and no condensation), initial and boundary conditions, and prescribed use of the standard high-Reynolds number k- $\varepsilon$ turbulence model. The main specifications for the CM were:

- Geometry: the upper man-hole and the upper, straight section of the injection pipe had to be represented. The pipe was supposed to be only a hydraulic obstruction and not to participate in heat transfer processes.

- As regards walls, the model should include: the heat capacity of the walls and obstruction plate; heat transfer between fluid and vessel walls and obstruction plate; heat losses from the outer side of the vessel walls, assumed to be uniformly distributed over the entire outer heat transfer surface of the vessel. The model (including insulation or not) shall be set to produce the correct heat flux (provided in the specifications) at nominal wall temperature $\left(107^{\circ} \mathrm{C}\right)$.

- No condensation and no radiative heat transfer should be considered.

- Turbulence: standard high-Reynolds k- $\varepsilon$ turbulence model should be used (with full treatment for effect of buoyancy on turbulent kinetic energy and dissipation rate). Turbulent Schmidt number: 0.8; turbulent Prandtl number: 0.9. Flat profile for velocity and turbulent intensity at the exit of the injection pipe. Turbulence intensity at the pipe outlet: $4 \%$. Molecular diffusion (Diffusivity between 1 and $\sim 3 \mathrm{~cm}^{2} / \mathrm{s}$ at $\mathrm{p}=1.3 \mathrm{bar}$ and $\mathrm{T}=388 \mathrm{~K}$ ) should also be considered. 
- Initial and boundary conditions: initial gas and wall temperatures: $107{ }^{\circ} \mathrm{C}$; experimental vertical distribution for gas concentrations. Experimental values for pressure, and steam mass flow rate and temperature.

The effect of buoyancy on turbulence parameters was prescribed because it is known that the effect of turbulence production due to buoyancy can be quite important for mixing transient involving large density differences (e.g. Visser et, 2012). However, the role played by buoyancy in the dissipation rate equation is controversial in literature, and therefore no recommendation was made for the parameter entering in the equation for $\varepsilon$, also because in some codes the default cannot be easily modified by the users.

The participants were expected to deliver the times of the erosion progression, the time evolutionhistories of helium concentrations and gas temperatures at selected locations, as well as axial and transversal profiles of gas temperature and velocity at various elevations, at selected times. Moreover, the organisations delivered an accompanying document with some information on physical and numerical models.

Not all organisations submitted both sets, and two organisations submitted results with two different codes. There were thus 13 contributions with the CM and 10 with the BEM. Table 1 summarises the submissions. In table 1, only the code used the total number of cells in the mesh, the main deviations of the CM from the specifications and the differences between CM and BEM are listed.

\subsection{Results with the Common Model (CM)}

Table 1 shows that various codes (mostly commercial CFD codes) have been used, with a broad spectrum of meshes, from 20000 cells to more than 1 million. The CM simulations were mostly performed with models complying with the specifications, the only important deviations being those referring to initial and boundary conditions. The non-obvious effect of small differences in boundary and initial conditions was not anticipated by some users. This is a good example of the importance of the benchmarking activities for establishing CFD modelling strategies to be used for the analysis of new problems. Therefore, the comparison with data of the simulations using inaccurate values of steam injection temperature and initial helium concentration profiles is somewhat affected by this deviation in the test conditions. For these contributions, the discrepancies with the data and other simulations are easy to explain. However, also for the other contributions, a very broad variety of results has been obtained. Figure 3 shows the time history of the erosion progression and, as example of gas temperature time evolutionhistories, the results for one elevation. It can be observed that the mixing time (600 s in the experiment) is well predicted in only three simulations (AERB, IBRAE2 and PSIF), whereas most submissions exhibit either a strong underprediction or overprediction. 
Considering the essential elements of the setups for the simulations (Table 1), but also the additional information provided, it is not obvious how to associate the success of the predictions to any specific difference between models. Since the physical models (and their implementation in the codes) must be very similar for all simulations, the differences in the prediction can enly be due be attributed to the mesh (refinement in certain regions and mesh topology), numerical methods, and other effects and modelling options not considered in the specifications (e.g. wall treatment, buoyancy effect on turbulence dissipation rate). Although previous comparison exercises for basic conditions displayed large difference in simulations using the same turbulence model (e.g., Gallego et al. 2007), iIt is therefore surprising in this benchmark that models with largely different number of cells lead to comparable predictions, and, vice versa, the use of detailed models with similar number of cells result in very large differences. Although post-test analyses (see below) shed light on some of these apparently puzzling results, the observed spread suggests that the set-up of a CFD simulation for a new problem is not a trivial task, and requires a careful evaluation of the applicability of previous experience for different geometrical configurations and physical conditions.

Another interesting observation concerns the temperature rise: in all simulations (and at all elevations) this is sharper than in the experiment, with the asymptotic temperature being much higher than the measured value (see Fig. 2). The temperature trend therefore reveals a systematic difficulty to predict the erosion process, which is not shown in most concentrations evolutionhistories (Fig. 4) where, because of the low data frequency, the calculated sudden drop seems to be always in agreement with the experimental results. The temperature distribution above the plate is, in general, reasonably well predicted in most simulations, where the horizontal profiles, in agreement with the experiment, show low peaks along the axis. Figure 5 shows, for example, the profile at some distance downstream the plate (Level G, z=5.63 m). However, with the exception of one simulation, the profiles are systematically shifted towards higher values, indicating that some mixing and/or heat transfer mechanism (convective or radiative heat transfer) is not well predicted. On the other hand, the small heat losses did not play an important role on the short time scale of the experiment.

Indeed, it seems that most simulations over predicted the gas temperatures already in the region below the plate. Figure 5 shows the temperature horizontal profile just below the plate (Level $\mathrm{H}, \mathrm{z}=4.97 \mathrm{~m}$ ) at $150 \mathrm{~s}$ : the temperature drop between the pipe outlet and the plate along the axis is underpredicted. This discrepancy, in principle, could be due to the fact that the codes predict perfectly symmetric flow, whereas in the experiment the jet was not perfectly centred due to flow non-symmetry at the pipe outlet, and thus the measured temperature does not represent the value (the maximum) at the jet impingement point. On the other hand, the deviation could reveal unexpected difficulties in predicting the broadening of the free jet. This question has been tackled in the open phase of the benchmark (Section 4). 


\subsection{Results with the Best Estimate Model (BEM)}

The simulations with the CM provided two main results: 1) even using very similar models (apart from the mesh) very different results can be obtained, in spite of the use of meshing strategies that could be considered "qualified" on the basis of previous experience with various tests in PANDA; 2) large discrepancies between calculated and experimental results were obtained. With respect to this second result, the agreement was expected to improve with the use of the BEM, where the participants could make use of the best modelling approach according to their experience. This mostly resulted (Table 1) in the use of a different turbulence model, the consideration of radiation heat transfer, and initializing the simulations with the experimental gas and wall temperature distribution. It is here appropriate to note that the steam in the mixture (absorbing, scattering and emitting infrared radiation) behaves as a participating medium (Abu-Romia and Tien, 1967; Howell, 1988), and therefore the radiation models consider not only heat transfer between solid surfaces, but also between gas and wall and within the gas.

Figure 6 shows the results for the erosion progression (calculated from the temperature rise) and the ime-evolutionhistory of the helium concentration at the highest elevation. Comparing results with those shown in Figs. 3 and 4 for the simulations with the CM, it can be noted that in a few cases the BEM results are better than the CM results (especially in the case with the largest discrepancy the use of different models produce a dramatic improvement, which was discussed in Andreani et al., 2016b), but in some cases the discrepancies are larger than using the CM. Three results are rather surprising: 1) the spread of results is still quite large; 2) the "best" results are obtained with a rather coarse mesh (163000 cells); 3) two simulations with identical code and very similar models (GRS and FZJ), which using the CM exhibited very different results, are now nearly coincident. This shows that the effect of the mesh possibly depends on the interaction with other modelling choices (turbulence model, numerical methods, boundary conditions, etc.).

It is also interesting to observe that the three simulations where radiation heat transfer was modelled result in a noticeable speed-up of the erosion process, due to the generation of additional convective motions in the upper part of the vessel, which enhance the mixing (Kelm et al. 2016b). For one simulation (CIEMAT), the faster mixing is uniquely due to radiative heat transfer, since the turbulence model was not changed in the BEM with respect to the CM. Considering radiative heat transfer helped in improving the prediction of the gas temperatures at some elevations but not at other ones. Moreover, the results for wall temperatures are somewhat contradictory, because the simulations using radiative heat transfer show qualitatively different results. This indicates that the simulation of radiation heat transfer (in combination with other models, first of all turbulence) requires additional 
investigations. The possible role played by radiation heat transfer in the process of gas mixing has, among others, also been recently suggested by Filippov et al. (2017).

\subsection{Conclusions and open questions originating from blind simulations}

Both sets of submissions, i.e. those using thea "common model" (CM) and those using the a "best estimate" model(BEM), produced a large variety of results. Especially the large differences in the results with the $\mathrm{CM}$ were not expected because, in order to establish some means for comparing the results, a comprehensive set of specifications have been recommended. The open phase of the benchmark was then considered necessary to provide more information on effect of meshing strategies.

The comparison between predicted and experimental results, as well as between simulations, raised a few questions about the actual importance of considering radiative heat transfer, the relation between mesh topology and other modelling aspects (turbulence model, numerical methods, boundary conditions, etc.), and the effect of some test conditions that were not modelled (e.g. non-symmetry of the flow at the outlet of the pipe).

The most important difficulty in interpreting the results and finding the reason of the large deviations was the lack of information on the velocity field downstream of the obstruction. In fact, the interaction between the jet and the density stratification is expected to be controlled by the velocity distribution at the interface between the rising jet and that interface (Paladino et al., 2013). Since the velocity field changes in time, due to the upwards movement of the leading edge of the jet, a meaningful comparison would require a time-dependent experimental information. Unfortunately, due to the short duration of the test, only long-term averages (on the time scale of the experiment) can be generated, and the comparison between the requested calculated instantaneous velocity profiles and these averaged values could not provide any hint on the fidelity of the simulation. One of the main difficulties to interpret the large spread of the erosion times is thus the lack of experimental information on the flow field, not only because of the problems discussed above but also because this is only available for a limited PIV area, which is at large distance from the obstruction. Therefore, an open benchmark using only the experimental information of test HP1_6_2 would lead to little progress in the understanding of the erosion process above an obstruction. The availability of velocity information under quasi-steady state conditions collected in auxiliary tests (Section 4.1) without helium ("zero" tests), but with the same geometrical configuration, permitted, however, to obtain new information to be used in the evaluation of the calculations. In fact, although, in principle, one would need information on flow patterns and temperature field below and above the plate for the specific 
test, some progress in the analyses could be expected from the use of information on the extended region above the plate provided in these "zero" tests.

It was then agreed that a meaningful open benchmark could be run, but this should include the analyses of the data on flow obtained in auxiliary tests, and should be organized in a number of steps (see next chapter), to gradually build confidence in the models used. All data were available to the open benchmark participants.

\section{THE OPEN BENCHMARK}

To help resolve the questions resulting from the blind phase, an open phase of the benchmark was conducted, where the extended data from the auxiliary "zero" tests (for similar conditions but without helium layer) were provided to the participants to permit a more basic validation of their models, by comparing the results for the flow downstream of the obstruction, which, in turn, are affected by the accuracy in simulating the free jet below the obstruction. A "cascade" of simulations was thus proposed, which aimed to separate the validation of the modelling approach for representing pure fluid dynamic phenomena from the application of the selected methodology to test HP1_6_2, where also heat/mass transfer effects play an equally (or even more) important role.

For each step, a summary of the contributions will be presented in tables, which include only the main information on the mesh and modelling options. The tables include the equivalent CPU time using one processor to provide a unified estimate of the computational overhead. A more complete list of aspects considered in the simulations and details on model selection is included in the Benchmark Report (2018).

\subsection{The "zero" tests and the multi-step open benchmark}

The two configurations (and PIV windows) for the tests with obstruction are shown in Fig. 7. In the complementary, "zero", tests, the geometry was exactly the same as for Test HP1_6_2, the only difference being the initial and boundary conditions. Additionally, it is noted that for the "zero" tests the zone covered by PIV measurements spans a much larger zone, extending $2 \mathrm{~m}$ above the obstruction. 
These tests were conducted injecting a constant steam flow rate in initially steam-filled vessels. In two tests (HP1_6_0 and HP1_7_0), pure steam was injected in steam-filled vessels, and the pressure was kept constant. The only difference was the flow rate.

These two tests, which were expected to deliver complementary information with respect to Test HP1_6_2, were used for the benchmark. A third test (HP1_8_0), which was performed with air and steam, and rising pressure was not used. For the two tests HP1_6_0 and HP1_7_0, the following nominal conditions apply:

- Pressure: 1.3 bar

- Initial steam and wall temperature: $108^{\circ} \mathrm{C}$

- Injected steam temperature: $150{ }^{\circ} \mathrm{C}$

- Steam flow rate: 60 g/s (HP1_6_0); 30 g/s (HP1_7_0)

Although test HP1_6_0 was the only with the same boundary conditions as HP1_6_2, the analysis of second test was also included, because in test HP1_6_0 temperatures were not recorded.

The open phase of the benchmark thus consisted of a number of steps, aiming to separate the qualification of the fluid-dynamic models from the validation of the full models for test HP1_6_2, for which the success of the predictions also depend on the appropriate representation of heat and mass transfer processes. The open phase of the benchmark was thus composed of four sets of results:

1) prediction of vertical velocity and turbulent kinetic energy (TKE) distributions for Test HP1_6_0 using a "common model" (CM);

2) prediction of vertical velocity distributions and temperatures for test HP1_7_0 using a "bestestimate model” (BEM);

3) prediction of vertical velocity, turbulent kinetic energy and temperatures (although not measured) for test HP1_6_0 using the BEM;

4) post-test simulation of test HP1_6_2 with BEM, including the same mesh for the plate region used for Step 3

Additionally, also the analysis of SETH-2 test ST1_2_2 (Erkan et al., 2009; Paladino et al., 2013), with the same initial and boundary conditions as for test HP_6_2, but without obstruction, was included as Step 5, to verify the capability of the models to properly predict the erosion process in absence of the obstacle. This step was optional for participants. 
The test configurations, and simulation requirements for the various steps are summarized in Fig. 8.

\subsection{Step 1 (analysis with CM of test HP1_6_0, where temperatures were not measured)}

The first step of the open benchmark was the analysis of test HP1_6_0 (with the same flow rate, 60 $\mathrm{g} / \mathrm{s}$, as in test HP1_6_2), using the CM model, i.e., using a common set of recommendations regarding modelling selection and initial and boundary conditions:

- In order to isolate possible spurious sources of deviations in the final Step 4 (post-test calculation of Test HP1_6_2), also test HP1_6_0 had to be run using the full geometrical model, including the straight portion of the inlet pipe

- Use of prescribed I.C. and B.C.:

- Initial fluid and wall temperature: $107^{\circ} \mathrm{C}$; Pressure and inlet flow rates: experimental curves. Flat pipe outlet velocity profile. Pipe exit turbulence intensity: $4 \%$.

- Fluid temperature at pipe outlet: $142{ }^{\circ} \mathrm{C}$ (precise value not critical, but important)

- Obstruction plate heat capacity included.

- Heat losses, condensation and radiative heat transfer neglected. Wall heat transfer to be considered: inner surface wall temperature to be prescribed (constant, at $107^{\circ} \mathrm{C}$ )

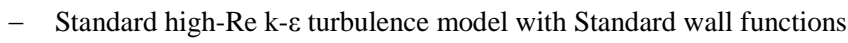

_ Value of $\mathrm{y}^{+}$in the cells below the plate between $\mathrm{R}=3$ and $10 \mathrm{~cm}$ around 30.

- Recommended simulation time: $500 \mathrm{~s}$

The summary of contributions is listed in Table 2

It is noted that most organizations contributed results using a finer mesh than that used for the blind benchmark, two used the same mesh, and one used a coarser mesh. Some of these choices are justified in the Benchmark Report (2018). None of the submitted contributions was based on a comprehensive sensitivity study aiming to obtain mesh-insensitive results.

The first variables to be considered in the comparison between data and calculated results are horizontal and vertical profiles of vertical velocity, extracted from the velocity field. Figure 98 shows the experimental information (averaged over 205 s), along with the elevation of the selected horizontal profiles. Figure $\underline{9} 8$ also includes the experimental profiles of axial velocity, maximum of 
the vertical velocity and difference between the maxima in the left and right half-plane in the PIV window (this last being and indicator of the non-symmetry of the flow past the obstruction). $\mathrm{Y}_{1}$ and $\mathrm{Y}_{2}$ are the elevations where the vertical velocity changes sign and the maximum coincides with the axial value, respectively. The former is the height of the recirculation zone (bubble) downstream of the obstruction; the latter indicates the position where the flow recovers the structure of a full jet (with maximum in the centre).

Figure 109 shows the calculated results for the vertical profiles of the axial velocity and the maximum vertical velocity. The comparison of the axial profile below the plate (between 4000 and $5000 \mathrm{~mm}$ ), although no data are available for evaluating the quality of the predictions, is of some interest because it shows that very large differences (also qualitative) exist between the simulations of CIEMAT and BARC and the other ones. However, the differences are substantially reduced in the region downstream of the plate, with maximum values being in a band of $0.25 \mathrm{~m} / \mathrm{s}$, which indicates a relatively small effect of the predictions below the plate and that most differences above the plate originate from the representation of the flow obstruction region. In fact, simulations showing the same flow development below the plate (S/NRA/R, KAERI and FZJ), diverge above the plate. The simulation of FZJ produced the highest values of the velocity at the top of the measurement region. It is also interesting to observe that the velocities are still growing with distance at the top of the measurement region, whereas the experimental values reach the maximum at lower elevation (between 6000 and $6500 \mathrm{~mm}$ ). Finally, the module of the minimum velocity in the "bubble" above the plate is underpredicted in all simulations. The vertical distribution of the maximum velocity also shows a similar spread of results, with all calculations underpredicting the distance from the plate where the maximum attains its highest value. Two calculations also overpredict the highest values.

The comparison of the velocities is to some extent affected by the still slowly evolving flow field in some calculations, which is well recognized in the evolutionhistory of the maximum velocity shown in the Benchmark Report (2018), which is not constant at the end of the simulation. In the experiment, however, the overlapping of the long-time average central profile using the entire data set with results using sub-sets indicates a practically steady-state condition. The reason for the evolving flow field is the slow fluid temperature increase, possibly due to the underprediction of heat transfer with the structures.

However, considering that some differences in the velocity distribution originate from the way the experimentally unknown temperature field is predicted, the agreement between predictions is reasonably good. The differences are larger for the Turbulent Kinetic Energy (TKE) in the recirculation zone (Fig. 11ㅂ), whereas in the developed flow region the simulations converge to the experimental value. The large (also qualitative differences) in the distributions are thus confined to the recirculation region, and decay with distance from the obstruction. 
An important feature of the transient that could not be investigated in test HP1_6_2, but could have an effect on the interaction of the jet with the density interface, was the symmetry of the flow structure and the flow evolution downstream of the plate. Asymmetric flow could either be produced by the impingement or directly originated at the outlet of the pipe. Whereas the flow below the obstruction cannot be observed also in the "zero" tests, its structure above the plate could be characterized, and it is reasonable to assume that it is representative of that produced in the reference test with helium. Therefore, an important aspect of the assessment of the models using the data from the zero tests is to verify whether the models correctly predict non-symmetries (if any) in the flow. For Step 1, since the recommendations included the modelling of the straight section of the pipe only, the verification only regards the production of non-symmetries in presence of axis-symmetric injection conditions.

Figure 121 shows the difference between the maxima in the two half-planes of the PIV window, which has a maximum in the zone close to the obstruction and vanishes within half a meter downstream of it. Most simulations do not display any substantial asymmetry in the flow field past the plate. Only the contributions from AERB and BARC present a qualitatively important deviation from symmetry, the former in the recirculation zone, the latter a slowly increasing difference with distance from the plate. This asymmetry could have an effect on the erosion process, and therefore on the interpretation of the results provided by AERB and BARC. Figure $1 \underline{2} 1$ also shows the elevation where the flow recovers the jet structure (with maximum velocity in the centre). It can be observed that the predictions, although obtained with the same turbulence model, are in a large band. Considering the 2-D velocity maps provided by the participants (Benchmark Report, 2018), however, it is presumed that at least a part of the differences could be due to the different criterion used to determine the condition of "equality" between axial and maximum vertical velocity.

It has already been observed that large discrepancies exist in the recirculation zone between calculations and experiments, and between the calculated results. This is more evident from the-time evolutionhistory of the minimum velocity (Fig. 1르), which shows underprediction in all simulations. It is surprising, however, that, in spite of the large differences in the velocity profile, the height of the recirculation zone is well predicted in all calculations.

Finally, the horizontal profiles of velocity and TKE at the two elevations indicated in Fig. $1 \underline{10}$ are shown in Figs. 143 and 1ㅁ4, respectively. With respect to velocities, at the elevation of the middle of the recirculation zones, with the exception of a narrow region around the axis, the simulations are in agreement with each other, and also in agreement with the measurements. A large spread of results, however, is exhibited by the turbulent kinetic energies, as regards both maximum values and profiles.

Similar considerations also apply to the elevation at large distance from the obstruction, where the velocities are in good agreement (especially outside the jet core), but the calculated TKE shows a large variety of values and horizontal profile shapes. 
These profiles indicate that even using the same turbulence model, the results can be quite different, depending on other modelling choices. Large difference between the results obtained with GOTHIC and those produced by CFD codes had to be expected because of the large differences in mesh detail (Table 2). The differences between CFD codes, however, are more difficult to justify, also because no correlation can be easily established between number of cells and main features of the calculated profiles. These results suggest that the mesh topology (and possibly details of the numerical model) could play an important role.

The general remark on the results of Step 1 is that even for the simplified conditions of HP1-6-0, important differences are observed, which are not related to turbulence modelling (the same standard $\mathrm{k}-\varepsilon$ for all) or radiation heat transfer (not considered), but probably originating mostly from the mesh (and topology) and, to a lesser extent, heat transfer and implemented boundary conditions.

Another observation, important for the interpretation of the results for the benchmark test (HP1_6_2), is that the differences in the erosion rates are likely to originate from a different prediction of the fluid dynamics below the density interface, and not related to the representation of the interaction region.

\subsection{Step 2 (analysis with BEM of test HP1_7_0, where temperatures were measured)}

Since temperatures were not recorded for Test HP1_6_0, and models constructed for best estimate simulations of the benchmark test should be validated also for their capability to properly predict fluid temperatures, the analysis of test HP1_7_0 is considered, although the steam mas flow rate was the half of that in test HP1_6_2 (30 g/s instead of $60 \mathrm{~g} / \mathrm{s})$.

In this test, the Reynolds number was thus 13500 instead of 27000. Since the effects of the Re number, at least on free jet flow development, are significant up to Reynolds numbers around 10000, and the TKE decay downstream of the plate is similar in the two tests, HP1_7_0 could be used for developing the BEM modelling approach to be used for HP1_6_0 (and HP1_6_2). In particular, in the multi-step approach for the open benchmark, the comparison of the results with test HP1_7_0 was intended to provide the validation of the models with respect to representation of heat transfer.

Table 3 reports the main features of the simulations contributed to the benchmark. Unless otherwise noted (FZJ and VTT), the mesh used is the onesame as that used for Step 1. Most users adopted the SST turbulence model, one the RNG model and some retained the standard turbulence model choice. With respect to Step 1, other important model modifications in some contributions were the consideration of radiation and the velocity profile at the pipe exit pipe (obtained by representing the straight section of the pipe or prescribed on the base of off-line calculations of the flow inside the 
entire pipe). Buoyancy effects on turbulence parameters were included in all simulations. For codes that do not offer this option in connection with certain turbulence models (e.g. SST), the buoyancy term has been added using external functions. These effects are also considered for all following steps.

Figure $1 \underline{6} 5$ shows the experimental flow field and the vertical velocity profiles to be used for the comparison. It is noted that both the height of the recirculation bubble and the height at which the flow recovers the full-jet structure are smaller than for Test HP1_6_0.

Figure 176 shows two vertical profiles of vertical velocity: the distribution along the axis and the profile of the maximum velocity. The general agreement between the various simulations is similar to that observed for Step 1, and, in the region above the plate, better for the simulations that exhibit a close agreement below the plate (KAERI, S/NRA/R and FZJ). However, the flow evolution below the plate has a minor effect on the velocity field above the plate. These results suggest that actually the jet "loses the memory" above the plate.

Underpredictions in the far-field velocity larger than for Step 1 are observed in the results of CIEMAT. Worse results were also obtained by VTT. Although the changes in modelling certainly play a prevailing role, and some mesh modifications with respect to Step 1 complicate the picture, this result possibly indicates that the disagreement between simulations does not depend on the jet Reynolds number. Moreover, the interpretation of the results is complicated by certain instabilities and large asymmetries in the velocity field which were exhibited by some simulations, but not observed in the experiment (Benchmark Report, 2018). Some of the calculated asymmetries appeared already at the pipe outlet, produced by bent in the lower part of the injection pipe (Figs. 1 and 7). However, PIV measurements immediately above the pipe exit performed in other HYMERES experiments for similar conditions (Paranjape et al., 2018) indicated that a well-developed turbulent pipe profile existed at the pipe exit. Although at the higher velocity of Test HP1_6_2 the effect of the bent could in principle be larger, the considerations above related to test HP1_7_0 suggested that one of the open issues of the blind benchmark, namely the effect of possible non-symmetries at the pipe exit the flow development above the obstruction, could be considered of minor importance for the evaluation of the results of the benchmark test. Detailed discussions on these and other aspects of the flow are discussed in the Benchmark Report (2018).

Concerning the fluid temperatures, the-time evolutionhistories isare presented and discussed in the Benchmark Report (2018). Here, we just mention that the two simulations including radiation heat transfer (FZJ and BARC) correctly predict the short time required for the fluid to reach equilibrium values with the structures, whereas the other predictions show still increasing temperatures at $1000 \mathrm{~s}$. It can be observed that these two simulations exhibit the most accurate predictions for the axial temperature profile (Fig. 1 187 ). The largest deviations occur at the position close to the plate, due to 
the difficulty to correctly predict the details of the flow within the recirculation zone (Benchmark Report, 2018).

Concerning the radial profile at Level D (only this one was required in the benchmark specifications), both simulations show a too narrow profile (but this is true for all simulations) and underprediction of the temperatures at some distance from the axis (Fig. 1ㅁ7). Although the results of BARC are probably somewhat biased by a large (and difficult to explain) temperature drop below the plate and the radial distribution at $1000 \mathrm{~s}$ calculated by FZJ is affected (Benchmark Report, 2018) by a plume oscillation (still of small amplitude at level D), it can be concluded that the two simulations agree with each other with respect to the effect radiation heat transfer can have. Globally, these simulations can also be regarded as the most successful, since the contribution of VTT (which does not model radiation) also shows good agreement above the plate, but these good results are due to the lower injection temperature (it can be noted in the axial temperature profile, Fig. 1ㅁ7).

\subsection{Step 3 (analysis with BEM of Test HP1_6_0)}

Step 3 was intended, in the multi-step approach of the open benchmark, to provide the participants the opportunity to validate the model selection made for test HP1_7_0 for the same flow as used in Test HP1_6_2, and to refine the mesh, if needed. In Step 3, additional horizontal profiles of velocity and TKE at low elevations were requested.

The overview of contributions for Step 3 is presented in Table 4 .

Figure 198 shows the axial and maximum velocity vertical profiles. For convenience, the results obtained for Step 1 are also displayed using the same scales.

Two participants (BARC and CIEMAT) used the same mesh as for Step 1, whereas the other four users used slightly finer meshes, adopting modifications that were suggested by the simulation of Step 2. Four participants used the same model settings as for Step 2, but CIEMAT and VTT used different turbulence model (or corrections).

Figure 198 shows that differences in injection velocity for five participants (not considering the contribution of CIEMAT, which for Step 1 used a too low value) are now more pronounced than for Step 1, due to the different assumptions for steam injection temperature (prescribed or calculated) and, possibly, details of the mesh used for the pipe. The development of the flow immediately above the pipe is also affected by the use of a velocity profile at the pipe exit, so that the profiles below the plate diverge more than for Step 1. 
In the region above the plate, results of BARC, CIEMAT, FZJ, and VTT are very similar to those obtained using the common model, and the results of S/NRA/R only show a noticeable difference for the maximum velocity. These results possibly indicate that the selection of the turbulence model, for these conditions, has a small impact on the prediction of the global features of the flow. The largest differences between Step 1 and Step 3 regard the simulations of PSI and CIEMAT, where, in both cases, the pipe exit conditions were strongly modified with respect to the calculations with the common model. The calculations of PSI with the "CFD like" GOTHIC code were "worse" than with the common model, but, due to the more physical representation of the pipe exit conditions (pipe exit velocity and temperature profiles are considered), are the best suitable to be compared with CFD codes in this benchmark.

The axial profiles of TKE (Benchmark Report, 2018) also show that the selection of the turbulence model (different from the standard $k-\varepsilon$ model in all calculations but that of PSI) has a strong influence in the region immediately above the plate, but its effect is substantially reduced already at relatively short distance from the top of the recirculation zone.

This consideration is especially true for the calculations of BARC, where the results with both standard and RNG model are nearly coincident. This is not expected, because the RNG model was developed to handle this kind of flows. The results of CIEMAT display the largest change, but for this simulation are not clear whether the large difference in mesh and inlet velocity were the most important effects. Finally, the radial profiles show that also the predicted axial values of S/NRA/R (for which no axial profile was provided) are less sensitive to the turbulence model selection than expected.

Obviously, these considerations cannot lead to the conclusion that the selection of turbulence model is immaterial for the representation of the flow past the obstruction and for the erosion process in transient test HP1_6_2, since an apparently small difference in the velocity and TKE distribution can have a large influence on the turbulent diffusivity distribution.

Indeed, the details of the results for Step 3 are also interesting in view of their prospective importance for the prediction of the benchmark test. Both velocity and TKE horizontal profiles show a large spread between the various simulations, and this suggests that the differences in the flow predictions could results in large differences in the erosion rate.

Finally, some considerations can be made on the predictions of fluid temperatures, although no experimental values have been recorded. It is observed that large differences exist in the free jet temperature decay (Fig. 2019), although the steam injection temperature is the same, apart from the calculation of VTT. Moreover, the radial profiles, similarly to the results for Step 2, show that radiation heat transfer has a strong effect on temperature spatial evolution. It is also interesting to 
mention that the time to reach a quasi-steady-state condition is very different in the various predictions (Benchmark Report, 2018) and, for some simulations, do not appear consistent with the results (also experimental) obtained for test HP1_7_0 (Step 2). In consideration of similar differences between the various simulations observed for test HP1_7_0 (where experimental values were available), it can tentatively be concluded that the modeling of radiation also permits a better representation of the time development of the temperature field.

\subsection{Step 5 (analysis with BEM of test ST1_2_2, without plate)}

Before the post-test results for the benchmark test are discussed, the analyses of test ST1_2_2 are briefly illustrated. The analyses of this test (conducted with the same configuration used for HP1_6_2, with the only difference that no obstruction was present), were expected to offer the opportunity to validate/improve the mesh outside the region of the obstruction plate, also taking advantage of the PIV measurements at short distance from the pipe exit.

The configuration of test ST1_2_2 (Erkan et al., 2009; Paladino et al., 2013) is shown in Fig. 210, where the initial distribution of helium in the injection vessel is compared with that present at the start of test HP1_6_2. Figure $2 \underline{1} \theta$ also shows the comparison of the other important boundary conditions, i.e. steam flow rate and temperature. The maximum helium concentration was slightly higher than in the benchmark test, whereas the steam injection temperature was lower. As for the steam mass flow rate, differences can be regarded as negligible.

It will be seen below that these conditions and the absence of the obstruction resulted in an about $30 \%$ faster stratification break-up.

Table 5 presents an overview of the four calculations contributed to this part of the benchmark.

Figure 221 shows the progression of the erosion, based on the gas temperature threshold of $115^{\circ} \mathrm{C}$. This limit is lower than for HP1_6_2 because the steam injection temperature was lower (Fig. 2110).

The simulations of the three organizations that submitted results also for the other steps (AERB, FZJ and PSI) accurately predict the erosion process, with some differences appearing only towards the end of the transient. The good predictions of these three contributions are also confirmed by the time evolutionhistories of the helium gas concentration (Fig. 2יz).

The only notable discrepancy in the three simulations are the delayed helium concentration drop calculated by FZJ at level D ( $\mathrm{z}=6.3 \mathrm{~m})$, which, however, may be due to a graphical representation effect, because (see Benchmark Report, 2018) the frequency of the required data (every 5 s) does not 
permit to display the calculated drop and recovery of the helium concentration during the time interval between two measurements.

Figure $2 \underline{4} 3$ shows the vertical velocity axial distribution and the horizontal profile at $\mathrm{z}=5003 \mathrm{~mm}$. It is observed that the calculation with GOTHIC (PSI) accurately predicts the maximum velocity at short distance from the pipe exit, whereas the other two simulations strongly overpredict it. The width of the profile instead is better calculated by the simulations of AERB and FZJ. It could be inferred that the velocity decay further up has been properly calculated in all simulations, because otherwise it would not be possible to capture the erosion rate. The analysis of the results of Step 4, however, will lead to different conclusions (see Section 4.6).

As regards the discrepancies at short distance from the pipe exit, although it can be presumed that they can partly be due to the slightly off-center position of the jet (in the third dimension), the results of AERB and FZJ suggest that either the pipe exit conditions or the near-field jet spreading is not properly calculated in their simulations.

This conclusion is supported by the observation of the axial profiles of gas temperature (Fig. 254) at two different times.

In both simulations of AERB and FZJ, the central temperature remains at the value of the injected steam up to above $5 \mathrm{~m}$, whereas the experimental value at that elevation has already dropped by more than $5 \mathrm{~K}$. In the calculation by PSI, however, the gas temperature drops smoothly to the lower measured value. The analyses presented in the Benchmark Report (2018) show that this result is due to the representation of both velocity and temperature profile at the pipe exit, due to the flow development inside the pipe and heat transfer with the pipe walls. In fact, if the velocity and temperature profiles are assumed flat, both axial velocity and temperature do not start decreasing below $5 \mathrm{~m}$.

It can be concluded that in the FZJ and AERB calculations either the entrainment in the free jet is too little, which produces a too small jet broadening, or the pipe exit conditions were not properly accounted for. For FZJ, the good agreement with the measurements for test HP1_7_0 (Step 2, half steam flow rate) with respect to both temperature (Fig. 1ㅁㄱ) and velocity profiles (Benchmark Report, 2018), suggests that the predictions are affected by the Reynolds number. The comparison between simulations for Test ST1_2_2 and HP1_6_2 (shown in the Benchmark Report, 2018) also indicates that the jet spreading below the plate depends on the evolution of the eddy diffusivity.

The elevation where the jet is fully developed (central temperature starts decreasing) is thus predicted at about $5000 \mathrm{~mm}$ and $5300 \mathrm{~mm}$ in the FZJ and AERB calculations, respectively. The 
underprediction of the jet spreading in the FZJ and AERB simulations can be recognized from the too narrow temperature profile, which is also present in the PSI calculation, although to a lesser extent.

This result suggests that also for Step 4 (to be discussed below) the simulations of FZJ and AERB should be affected by inaccuracies in the simulation of flow field and temperatures below the plate.

The axial temperature profiles show that only the calculation (FZJ) considering radiation heat transfer captures the correct level of temperature at large distance during the erosion process (time $<500 \mathrm{~s}$ ), although the temperatures drop between the elevation where the jet is fully developed and the bottom of the helium layer is overpredicted. In general, also the horizontal temperature profiles (Fig. 26도) show a better success of the FZJ simulation, although not all results seem to offer a consistent picture (Fig. 276).

From the profiles in the mixed region below the tip of the jet (Benchmark Report, 2018) one can conclude that the modelling of radiation heat transfer was necessary to correctly calculate the fluid temperatures. Also at higher levels (Fig. $2 \underline{6} 5$ and 276), where the upwards propagation of the jet produced the temperature rise and helium concentration drop, the temperatures before the mixing (Level D) or during or immediately afterwards (Level C) are much better predicted by FZJ. At times after the mixing has occurred (250 s at level D and especially at $500 \mathrm{~s}$ at level C), however, the calculation of FZJ shows notable discrepancies, possibly due to the (small) asymmetry in the flow and temperature field appearing shortly before the dissolution of the helium layer is completed (Benchmark Report, 2018).

Some additional information can be obtained on the gas temperature-time evolutionhistories at the required positions (not shown). First of all, the three simulations of AERB, FZJ and PSI correctly predict the temperatures in vicinity to the pipe exit, which shows that the correct boundary conditions were applied and no numerical diffusion corrupted the simulations from the injection elevation. The best predictions were obtained by FZJ using radiation, although the results of PSI using a coarse mesh and a standard correlation for convective heat transfer are nearly equally successful.

The results for off-axis positions above the initial density interface are somewhat contradictory, with generally better results obtained by PSI, with the effect of radiation being to excessively reduce the temperatures during the entire transient and also after the full dissolution of the helium layer. The discrepancy is especially large at some positions during the erosion process. On the contrary, at lower positions, the calculation with radiation permits to reproduce the correct temperature, whereas the calculation with GOTHIC predicts a strong superheat, the largest differences occurring during the transient compression and erosion of the helium layer, before mixing. Although it cannot be excluded that convective heat transfer could also play a major role in the different predictions (since both 
turbulence model and meshes were different), it can be presumed that the largest contribution to the divergent behaviour could come from the modelling of radiation in the simulation of FZJ.

Since all positions where the discrepancies are very large are all in regions of slow velocities and even in nearly stagnant zones, these result suggest that the strongest effect of radiation could be observed in the zones where the temperature increase due to compression of the fluid cannot be contrasted by the weak convective heat transfer but is kept low by the effect of radiation heat transfer. Moreover, the results indicate that the effect of radiation heat transfer may show up more distinctly during transients rather under quasi-steady state conditions (such as those established in Test ST1_2_2 after the dissolution of the stratification), which would be justified by the different time scales of the convective heat transfer (which is bound to the finite propagation velocity through the fluid) and radiation heat transfer (which instead has practically an immediate effect).

\subsection{Step 4 (post-test analysis of benchmark test HP1_6_2)}

The last step in the analysis is the post-test simulation of the benchmark test HP1_6_2. For visualisation purposes, the distributions of helium concentration and gas temperatures at various times calculated by one of the participants are shown in Figs. 28 and 29.

In accordance with the rationale for the multi-step approach, mesh around the plate and modelling would be expected to be the same as for Step 3 (HP1_6_0), with some corrections to the mesh in the main domain due to the knowledge gained from the analysis of Test ST1_2_2, featuring helium layer erosion without obstruction. Table 6, where the overview of the contributions is presented, shows that only FZJ and PSI ran all steps and followed this path in building the model used for the final calculation. Also BARC and S/NRA/R submitted results with same mesh and model setting, but opted not to perform the additional Step 5. It is also noted that one organization used a coarser mesh than for Step 3: CIEMAT model reverted to the coarse mesh model used for the blind benchmark.

Also with respect to the turbulence model, in some contributions Step 4 has not been analyzed with the same selection used for the other steps. As regards radiation heat transfer, it is noted that one contribution (VTT) used this model only for Step 4: therefore a preliminary validation using the data of HP1_7_0 an ST1_2_2 (Steps 2 and 5) was not performed.

Figure 3027 shows the erosion progression determined using the times at which for each location the gas temperature rises to $120{ }^{\circ} \mathrm{C},{ }^{1}$ whereas Fig. $31 z 8$ shows the helium concentration-time

${ }^{1}$ See Section 2.2. Results up to level B $(7500 \mathrm{~mm})$ are confirmed by the inspection of the temperature predictions. For level A (8000 mm), the "erosion time" is somewhat underestimated. 
evolutionhistory at level B $(\mathrm{z}=7.48 \mathrm{~m})$. In these figures, the results obtained in the open phase are compared with those submitted for the blind benchmark, using the best estimate model. It is noted that VTT did not contribute best estimate results for the blind benchmark, and thus no comparison is possible for this organization.

It is observed (Fig. $\underline{31 z 8}$ ) that several organizations that participated in the blind benchmark could obtain better results (especially BARC, S/NRA/R and GRS, with this third organization, however, supplying the final results with a model that was not verified in Steps 1 to 5). AERB, CIEMAT and FZJ, instead, practically obtained the same results as for the blind simulations. This outcome of the multi-step validation of the model for CIEMAT is obvious, because the final step was rum with the same mesh and model settings as for the blind exercise.For AERB (Benchmark Report, 2018), the results (using a much finer mesh) have been shown to depend on the mesh around the plate and therefore on an accurate prediction of velocity field near the plate (comparing the results of sensitivity studies for Step 3), overall grid size in the stratification region (Step 5), and numerical scheme. On the other hand, the Realizable k- $\varepsilon$ was preferred to the standard and the RNG model only for the better convergence obtained with this turbulence model. For FZJ, the results could seem somewhat surprising, because for all other steps the model delivered rather accurate predictions, at least at some distance from the pipe exit. It has to be considered, however, that the results of the blind benchmark have not been revised systematically applying the outcome of the other steps, since they used a Best Estimate Model constructed on the base of a comprehensive validation experience. Numerical effects were also carefully investigated by FZJ, but showed no considerable importance on global results.

A possible contribution to the discrepancy can be due to the underprediction of the jet broadening below the plate, as suggested by the results of Step 5, and/or the slightly larger maximum velocity and TKE at high elevations (observed in Step 3). The difference in the results can be explained neither by the modelling of radiation alone (also considered by BARC, CIEMAT, GRS, and VTT), nor by mesh or turbulence model or injection conditions. It is likely that a combination of all these elements finally produced the large differences observed in the final results of FZJ for the transient test. Especially interesting is the comparison between the calculations of FZJ and BARC, because for Step 3 the results of both simulations were close to each other, the only important differences being the TKE magnitude at some distance from the plate, and the elevation of the full jet recovery.

This suggests that the results for the erosion progression are strongly affected by the turbulence model, and wall layer treatment of the jet impingement zone (including the best choice for the size of the cell adjacent to the plate). Some additional elements to partly explain similarities and differences between results will be provided by the analyses of the flow variables (see below), but only further analyses and sensitivity studies by the individual organizations could bring some light on the role played by mesh and modelling choice. 
As for other simulations, the concentrations-time evolutionhistories confirm that FZJ and CIEMAT (and at late times also VTT) overpredict the erosion rate, whereas the others either capture or slightly underpredict the mixing rate. It is also observed that in the simulation of VTT, the erosion is initially slower (Level F, at $\mathrm{z}=6 \mathrm{~m}$ ), probably due to the delay in the reattachment of the flow above the plate (see below), which was observed for Step 2, but not for Step 3 (Benchmark Report, 2018).

As for the effect of modelling radiation (which, in the case of BARC lead to very accurate results, but, for FZJ resulted in too fast mixing), Fig. $32 z 9$ shows the comparison of the results obtained by GRS (Schramm et al., 2017) and VTT (Huhtanen, 2018). For this simulation, the accelerating effect of radiation is very large. In other investigations (e.g. Kelm et al., 2016b) for similar conditions, radiation was shown to promote mixing due to its influence on temperature (and density) fields, although its effect was not as substantial as in the simulations shown here. Additional studies on the effects of radiation are presented in the Benchmark Report (2018).

The apparently excellent global results obtained with GOTHIC and coarse meshes are probably affected by compensation of errors, as indicated by the comparison for the velocity.

Due to the large averaging time of the available velocity measurements, the comparison between the calculated axial and horizontal velocity profiles (Fig. $3 \underline{3} \theta$ ) has to be taken with some caution, as shown by the large fluctuations in the measurements over a $10 \mathrm{~s}$ period around the central time (300 s) of the averaging period. Nevertheless, it can be recognized that the velocity in the PIV window calculated by PSI is far too low, which suggests that the good predictions for the erosion result from the compensation of errors between the lower velocities in the far field above the plate and numerical diffusion. Since the results for Step 5 in the near field (without plate) were excellent and the results for Step 3 (without helium layer) were reasonably good, it can be concluded that the complexity of the transient test HP1_6_2 lead to overprediction of the velocity decay between the plate and the density interface. It can also be observed that the same order (from faster to slower) in the erosion timing displayed by concentration and temperature traces can be found in the magnitude of the velocity: the simulation of FZJ, which predicts fast erosion, also predicts the largest velocity in the PIV window. Vice versa, the simulation of AERB, which underpredicts the velocity, also slightly underpredicts the erosion rate.

A special attention deserves the simulation of VTT, which used a very detailed mesh and calculated (as result from the detailed modeling of the injection pipe) an asymmetric velocity and temperature profile at the pipe exit. The evolution of the calculated flow field (Benchmark Report, 2018) shows for about $200 \mathrm{~s}$ a splitting of the velocity field above the plate (which can also be inferred from the temperature distribution at 100 and $200 \mathrm{~s}$ in Fig. 28), and only at about $220 \mathrm{~s}$ the plume/jet structure is 
recovered (see temperature distribution at $300 \mathrm{~s}$ in Fig. 28). Since for the same flow rate (Step 3) this delay was not observed (but it was observed in test HP1_7_0, Step 2), the results suggest that the density interface produced the same effect as a lower mass flow rate. No conclusive explanation could be found so far for the results concerning the recirculation zone above the plate. On the other hand, the simulation of BARC (taken as example, but similar behavior is displayed by FZJ and CIEMAT) also show an "open” flow structure at early times, but already after $100 \mathrm{~s}$ the full jet/plume is recovered. This behavior does not depend on the steam injection temperature (constant at $150{ }^{\circ} \mathrm{C}$ in the BARC calculation).

The observation related to the VTT simulation is particularly important, because it suggests that the set-up of a model based on the good results for simplified conditions can prove not to be equally successful when applied to the more complex situation of a test featuring transient behavior and strong density differences.

Some important observations can be made on the axial and horizontal temperature distributions (Figs. $3 \underline{4} 1$ and 35z), Generally, the most accurate axial temperature distributions are obtained by the models including radiation heat transfer, but also the simulation with GOTHIC, which accurately accounts for the temperature drop between the pipe exit and the plate, displays a quite remarkable agreement. The initially too large temperature difference between the elevations below and above the plate calculated by VTT is related to the delay in the re-attachment of the flow (see above).

Due to the complexity of the phenomena, it is not possible to draw any conclusions, but it is simply observed that no obvious correlation seem to exist between accuracy in the calculation of the temperatures and mixing rate. The horizontal temperature profile immediately below the plate (Level $\mathrm{H}$ ) shows that all simulations predict a too narrow peak, with rather flat profile at some distance from the axis. At this elevation, the most distinct difference between some simulations including radiation (CIEMAT, FZJ and VTT) and those not representing this heat transfer mode is the larger temperature drop between the centre of the jet/plume and the periphery of the flow. This, however, is not observed in the calculation of BARC.

For the region above the plate, the results are obviously affected by the different progression of the stratification erosion. It can be observed, however, that at intermediate elevations (Levels G and D) most of the simulations including radiation predict better the temperature profiles, especially the temperatures near the wall.

The results obtained with the models with radiation at the bottom of the dome (Level C) seem to be less accurate than those neglecting this heat transfer mode. In fact, whereas at $250 \mathrm{~s}$ the top of the jet has already reached that elevation in the simulations of CIEMAT, FZXJ and BARC, and therefore the temperature profiles are not comparable, at $500 \mathrm{~s}$ in all simulations the mixing has been completed at 
level C, and one can observe large discrepancies with data in the simulations with radiation (again, excluding BARC), of the same magnitude, though opposite in sign, as the other simulations.

The simulation of FZJ at $150 \mathrm{~s}$, moreover, is affected by asymmetry, similar to that observed for test ST1_2_2. In this case, it is easy to explain this profile with the bending and fluctuation of the plume, as shown by the temperature field at various times (Benchmark Report, 2018). It is interesting to note that the presence of a density interface acts on the plume as a lower flow rate. In fact, large-scale oscillations were predicted for HP1_7_0 (30 g/s), but not for HP1_6_0 (60 g/s).

The comparisons of the gas temperature evolutionhistories at various positions (Benchmark Report, 2018) permitted a better evaluation of the role played by radiation. Especially interesting is the consideration of the temperature level reached during the transient, the temperature after the stratification moved above a specific location, and the temperature rise time at the various locations.

An example of temperatures at higher locations is shown in Fig. 3조. At the position along the axis (Fig. $3 \underline{6} 3$ left), the calculations can be divided in three groups: 1) the simulations with radiation are the most accurate with respect to the final values, but underpredict the times of the rise (erosion), with the exception of BARC; 2) the CFD simulations without radiation overpredict the temperature; 3) the results of PSI with GOTHIC are in between. Concerning the slopes of the temperature increase, the results are rather sparse, with GOTHIC obtaining better results at intermediate levels, and only CFD simulations with radiation (especially BARC) being able to predict the sudden rise at higher elevations.

As regards the off-axis positions initially immerged in the helium layer (Fig. 3즈, right), the two CFD simulations without radiation clearly overpredict the temperatures at all positions, whereas four of the CFD simulations with radiation (CIEMAT, FZJ, GRS and VTT) underpredict the temperatures to various extent. In general, the BARC simulation (also with radiation) is the most successful. The calculation of PSI, however, is nearly equally accurate.

Finally, the temperatures at two positions off-axis below the initial bottom of the helium layer are considered (Fig. 374). Close to the centre (325 mm form the axis), at the elevation of the recirculation bubble above the plate (GH_19, $\mathrm{z}=5.3 \mathrm{~m}$ ) the results are in a band of $15 \mathrm{~K}$, with the PSI results being the most accurate. Further off-axis (about $900 \mathrm{~mm}$ form the centre), and at the level of the injection, therefore at a position weakly affected by convective motions, the CFD simulations with radiation show substantially better results than the other calculations. At this position (K_17, z=4 m), and this was the case also for test ST1-2-2 (Benchmark Report, 2018), the role played by radiation in nearly stagnant region appear more clearly. It can also be shown that in these regions, where the heat-up is 
initially mainly caused by compression, radiation is the only effective heat transfer process, since it does not require the development of the boundary layer, which affects instead convective heat transfer (Filippov et al., 2017)

It must also be remarked that the various predictions including radiation heat transfer produced different results, as expected from the use of different models and methods to calculate the steam absorptivity. The differences, also connected to the different time progression of the erosion, are large during the mixing process, and vanish at many locations after the helium layer has been dissolved.

\section{CONCLUSIONS}

The experiment in the PANDA facility chosen for the present benchmark, test HP1_6_2, addresses the stratification erosion induced by a vertical steam jet, which originates from the exit of a circular pipe located below the bottom of the helium-rich layer. The mixing is somewhat slowed down by a small, horizontal, circular plate above the jet source. The benchmark consisted of two phases: blind and open. The results of the blind benchmark exhibited a large spread of results, some showing very large discrepancies with the measured data, which was not expected, especially for the part addressing the use of a "common" model. The results of the blind simulations made evident that further investigations and validation studies were necessary to separate different sources of errors and avoid their mutual elimination (compensating errors) in a complex model.

It was recognized that the most important difficulty in interpreting the results and finding the reason of the large deviations was the lack of information on the velocity field downstream of the obstruction, since only long-time averaged velocities were available in the region of the initial density interface, above $6 \mathrm{~m}$. It was therefore agreed that valuable information on the flow produced by the interaction of the free jet with the obstruction could be obtained from auxiliary tests without helium ("zero" tests), but with the same geometrical configuration and featuring an extended region above the plate where velocity measurements were available.

Therefore, the open benchmark included the analyses of the data on the flow structure above the plate obtained in these auxiliary tests: a "cascade" of simulations was thus proposed, which aimed to separate the validation of the modelling approach for representing pure fluid dynamic phenomena (using the data of the "zero" tests HP1_6_0 and HP1_7_0) from the application of the selected mesh and models to the more complex test HP1_6_2, for which the success of the predictions also depend on the appropriate representation of heat and mass transfer processes 
The open phase of the benchmark thus consisted of four steps, with an optional fifth stepAdditionally, also the analysis of SETH-2 test ST1_2_2, with the same initial and boundary conditions as for test HP1_6_2, but without obstruction, was included as Step 5, to verify the capability of the models to properly predict the erosion process in absence of the obstacle, and therefore test their basic capabilities to represent the global features of the transient in the entire flow domain. Finally, also the velocity measurements at the pipe exit in two other test series were used in some comparisons to verify the appropriate representation of the injection conditions.

Since each participant was requested to submit one set (best estimate) of final results for each step and only the main sensitivity studies of some organisations were contributed to the final report, only general conclusion will be discussed, leaving the detailed answers to the questions to future publications of the individual organisations.

The simulations with a common model for the quasi-steady state conditions of test HP1_6_0 without helium (step 1) were expected to provide the opportunity to investigate the effect of mesh on the simulation of the interaction of the jet with the plate. The final results submitted exhibited notable differences, especially in relation to transversal distributions and flow development downstream of the plate. Although the simulations could be affected by numerics and the spurious effects of the calculated slow change of the thermal field, the analysis indicate that meshing strategies could not converge to produce similar results and setting an adequate mesh for representing the flow modification due to an obstacle poses a real challenge. It can be inferred that the variety of results later obtained for the full test are strongly affected by the meshes used in this region. This is somehow confirmed by the observations that the few simulations of test ST1_2_2 (Step 5) without obstacle were all reasonably successful, although performed with largely different meshes and model selections.

As regards the interaction of mesh and model selection (which produced in the blind benchmark astonishingly different results using a turbulence model but not a different one), no new information could be generated within this benchmark, due to the lack of systematic analysis and the use of similar turbulence models (all variants of k- $\varepsilon$ and k- $\omega$ models). However, comparing results for test HP1_6_0 with the "common" model and the "best-estimate" model (Steps 1 and 3, respectively), it was observed that the selection of models had a smaller effect than expected, and was seemingly less important than the mesh.

Thermal radiation heat transfer was confirmed to have an accelerating effect on the progression of the erosion process, independently of the specific model used, and a substantial part of the information obtained from the temperatures indicates that this heat transfer mode should be modelled to get the correct spatial and temporal evolution of the thermal field. However, since various simulations with 
radiation were only performed for the final step, some results appear to be contradictory, and inaccuracies (possibly compensated or enhanced by radiation) are certainly associated with the use of meshes not optimised for the flow investigated (see above) and with the possible interaction with other models and effects, no firm conclusion could be reached on the necessity and approach to model radiation heat transfer. This issue will certainly require further investigations, both experimental and numerical.

No definite conclusion could be reached with respect to best choice for turbulence model, as the meshing appeared to be a more important issue for the conditions investigated in this benchmark.

The velocity and temperature distributions in the jet at the pipe exit have some effect on flow and thermal variables, but this is mostly confined to the region below the obstruction. Finally, the effect of numerical methods has been reported from some participants and for some contributions it can be suspected to be responsible for some anomalous results and the submission of the final results with meshes not optimised. However, the absence of systematic studies in most contributions show how difficult is to implement in the analyses of transients requiring hundreds hours of CPU a rigorous approach to guarantee mesh and time step independence.

The main conclusion of the open benchmark is that the step-by-step validation permitted some progress with respect to some of the items identified above. However, large discrepancies with data in the final analyses of the test are observed, which cannot be easily attributed to specific model deficiencies. The uncertainty is partly due to the difficulty to perform exhaustive analyses for each step including all effects, partly to the physical model limitations (e.g. use of RANS models for turbulence), and partly to specific features of the reference test that cannot be tackled in simulations of simplified conditions. On the one hand, even for the simpler fluid conditions of the tests without density interface, mesh and models could not be fully assessed. On the other hand, it is clear that the complexity of the physical conditions prevailing in the selected test, where the modification of the flow produced by the obstacle interacts with the stratification erosion process, rendered the splitting of the problem in hydrodynamic and heat/mass transfer components of lesser use than anticipated.

A few general considerations should be added in relation to the blind benchmark and the large, unexpected, differences in the results. The spread of the results was similar, for instance, to the recent OECD/NEA-PSI CFD benchmark without obstruction, although specifications for the "common" model were given, which covered various aspects of the simulation (geometry, turbulence modelling, initial, and boundary conditions, some fluid and flow properties, etc.). Since other aspects of the simulation were not considered in the specifications (e.g. wall treatment, buoyancy effect on dissipation rate), and therefore some of the differences could be due to specific code inputs as well as to the numerical methods used, and the level of validation (including mesh convergence studies and 
application of BPG) was different for the various contributions, it is difficult to draw any conclusions from the comparison of the requested results. However, it can be observed that, similarly to previous exercises for similar flows and configurations (but without obstruction), the present results suggest that whenever a new problem is tackled, established modelling strategies must be evaluated again. The outcome of the exercise reinforced the awareness of the spread of results that can be obtained if the adequacy of the mesh is solely evaluated on the base of previous experience and limited mesh refinement studies. In this respect, the fact that some of the best results were obtained with rather coarse meshes should not be used as argument in favour of this approach for applications, without appropriate validation. Furthermore, even the use of very detailed meshes, resulting from systematic studies (going close to the full application of the BPG) does not lead necessarily to similar results, which are possibly affected by the topology of the mesh, local refinement, details of the numerics, and other effects.

These considerations lead to a few key-findings of this comparison. First of all grid independence must be proven for each physical and geometrical model as well as set of boundary conditions and cannot be simply assumed that this can be concluded from a similar case. Small changes in the flow/setup, here the implementation of a small flow obstruction, may challenge the model validity range. This suggests that continuous validation and a backward assessment of previous results (e.g. those obtained for the OECD/NEA benchmark or other tests addressing stratification break-up) is necessary. The results furthermore highlight that the effect of user-defined numerical or physical assumptions is in the order of those of model differences.

In summary, the results highlight, for the sake of more precise conclusions, the need for proper grid convergence studies, beyond the prescriptions of the BPG (not addressing mesh topology), which possibly should be improved by including, for instance, recommendations on mesh structure for an open catalogue of simple flows. Moreover, following the practice of the V\&V community (e.g. that organised by ASME), in future benchmarks more attention has to be paid to code inputs and their effects on the numerical solution of the equations.

The results obtained by each participant using the best estimate models show that the combination of mesh and modelling approach again can result in a wide spread of results, with the quality of the results not always being improved using a model selection that proved to be successful for other configurations and test conditions. For instance, the use of refined turbulence models (such as SST) and considering radiative heat transfer did not result in fully satisfactory predictions, and made evident that further investigations and validation studies are necessary to separate different sources of errors and avoid their elimination (compensating errors) in a complex model.

It is important here to stress once more the importance of blind benchmarks, because they disclose the difficulty in tackling new problems for which the modelling strategy must be derived from previous 
experience. This is true, we believe, in general, but it is more true for containment related problems, because the strict application of BPG is hindered by the long computation times associated with complex geometries, large volumes and long transients.

Finally, considering the specific configuration of the test on which it was based, the present benchmark revealed the (partly unexpected) difficulty to simulate a flow in presence of a simple obstruction. This observation suggests that in the future more experimental data and associated V\&V will be required to validate the codes for more complex geometries. These issues are currently addressed in the OECD/NEA project HYMERES-2 (OECD/NEA, 2017). 


\section{ACKNOWLEGMENTS}

The experiment was conducted by the Experimental thermal-hydraulic group of PSI, composed by three of the authors - R, Kapulla, S. Paranjape and D. Paladino - and G. Mignot, M. Fehlmann, L. Ryan and S. Suter. The authors gratefully acknowledge the support of all the countries participating in the OECD/NEA HYMERES project and the OECD/NEA secretariat. The authors would like to thank all the members of the Management Board and the Programme Review Group of the HYMERES project for their help in defining the test programme and evaluating the test results.

\section{REFERENCES}

Abe, S., Studer, E., Ishigaki, M., Sibamoto, Y., and Yonomoto, T. (2018) “Stratification Breakup by a Diffuse Buoyant Jet: The MISTRA HM1-1 and 1-1bis Experiments and their CFD Analysis”, Nucl. Eng. Design, 331, pp. 162-175.

Abu-Romia, M.M. and Tien, C.L (1967) “Appropriate Mean Absorption Coefficients for Infrared Radiation of Gases”, Journal of Heat Transfer, 89(4), 321-327.

Allelein, H.-J., Fischer, K., Vendel, J., Malet, J., Studer, E., Schwarz, S., Houkema, M., H. Paillère, H., Bentaib, A. (2007). International Standard Problem ISP-47 on Containment Thermal Hydraulics. Nuclear Energy Agency, Committee on the Safety of Nuclear Installations, Final Report NEA/CSNI/R(2007)10, 2007.

Allein, H.-J., Reinecke, E.-A., Belt, A., Broxtermann, P., and Kelm, S. (2012) “Combined Analytical and Experimental Investigations for LWR Containment Phenomena”, Nucl. Eng, Technol., 44(3), 249-260.

Andreani, M., Badillo, A., and Kapulla, R. (2016a) "Synthesis of the OECD/NEA-PSI CFD Benchmark Exercise”, Nucl. Eng. Design, 299, 59-80

Andreani, M., Daqiang, Y., Gaikwad, A.J., Ganju, S., Gera., B., Grigoryev, S., Herranz, L.E., Huhtanen, R., Kanaev, A., Kelm, S., Kim, J., Nishimura, T., Schramm, B., Sharabi, M., and Paladino, D. (2016b) "Synthesis of a blind CFD benchmark exercise based on a test in the PANDA facility addressing the stratification erosion by a vertical jet in presence of a flow obstruction”, 
OECD/NEA 6th Workshop on Computational Fluid Dynamics for Nuclear Reactor Safety (CFD4NRS-6), MIT, Cambridge, MA-USA, 13-15 September, 2016.

Andreani, M. and Paladino D., (Benchmark Report, 2018), prepared by PSI (with appendices contributed by AERB, FZJ and VTT) (2018) “OECD/NEA HYMERES project: synthesis of results of the benchmark on PANDA test HP1_6_2”, PSI Technical Note AN-42-17-07 Rev.1, Project report HYMERES-P-17-48, May 2018.

Bentaib, A., Meynet, N., and Bleyer, A. (2015) “Overview on Hydrogen Risk Research and Development Activities: Methodology and Open Issues”, Nucl. Eng. Technol., 47, 26-32.

Breitung, W. and Royl, P. (2000) "Procedure and Tools for Deterministic Analysis and Control of Hydrogen Behavior in Severe Accidents”. Nucl. Eng. Design, 202, 249-268.

Chan, C.K. and Jones, S.C. (1997) “Gas Mixing Experiments in a Large Enclosure”, Proc. of the $18^{\text {th }}$ Annual Conf. of the Canadian Nuclear Society

Choi, Y.-S., Lee, U.-J., and Park, G.-C. (2001) "Study on local hydrogen behaviors in a subcompartment of the NPP containment”, Nucl. Eng, Design, 208, 99-116.

Deri, E., Cariteau, B., and Abdo D. (2010) “Air fountains in the erosion of gaseous stratifications”, Int. J. Heat and Fluid Flow, Volume 31, Issue 5, Pages 935-941.

Erkan, N., Mignot, G., Kapulla, R., Paladino, D., Zboray, R., Strassberger, H.J., Bissels, W. and Fehlmann, M. (2009) “OECD SETH-2 PANDA Test ST1_2_2 Quick-Look Report”, PSI internal technical report TM-42-09-07-0, April 2009.

Filippov, A.S., Grigoryev, S.Yu., O.V. Tarasov, O.V. (2017) “On the possible role of thermal radiation in containment thermal-hydraulics experiments by the example of CFD analysis of TOSQAN T114 air-He test”, Nucl. Eng. Design, 310, 175-186.

Gallego E., Migoya E., Martín-Valdepeñas, J.M., Crespo, A.,García, J., Venetsanos, A. Papanikolaou, E., Kumar, S., Studer, E., Dagba, Y., Jordan ,T., Jahn, W., Høiset, S., Makarov, D., Piechna, J., (2007) "An intercomparison exercise on the capabilities of CFD models to predict distribution and mixing of $\mathrm{H}_{2}$ in a closed vessel”, International Journal of Hydrogen Energy, 32, 2235 $-2245$ 
Gupta, S. (2015) “Experimental Investigations Relevant for Hydrogen and Fission Product Issues Raised by the Fukushima Accident”, Nucl. Eng. Technol., 47, 11-25.

Howell. J.R. (1988) "Thermal Radiation in Participating Media: The Past the Present, and Some Possible Futures”, Journal Heat Transfer, 110, 1220-1229.

Huhtanen, R. (2018), Private Communication.

Kapulla, R., Mignot, G., Paranjape, S., Suter, S., Fehlmann, M., and Paladino, D. (2015a) “OECD/NEA HYMERES Project: Jet/Plume interacting with flow obstruction HP1 Series. Test Series Report”, PSI internal report TM-42-15-16, Rev.0, Project report HYMERES-P-15-26, Nov. 2015 (report restricted to project participants).

Kapulla, R., Paranjape, S., Mignot, G., Suter, S., Fehlmann, M., and Paladino D. (2015b) “OECD/NEA HYMERES Project: PANDA Tests HP1_6_0, HP1_7_0, HP1_8_0, and HP1_678_Disk Data Report”, PSI internal report TM-42-15-13 Rev. 0, Project report HYMERES-P-15-25, October 2015 (report restricted to project participants).

Karwat, H. et al. (1999) "State-of-the-Art Report on Containment Thermal-hydraulics and Hydrogen Distribution”, OECD/NEA group of experts, CSNI/R(99)-16 (1999).

Kelm, S., Ritterath, M., Prasser, H.-M., and Allelein, H.J. (2016a) "Application of the MINIPANDA Test Case ‘Erosion of a Stratified Layer by a Vertical Jet’ for CFD Validation”, Nucl. Eng. Design, 299, 124-135.

Kelm, S., Müller, H., and Allelein, H.-J. (2106b) "Importance of thermal radiation heat transfer modeling in containment typical flows”, Paper submitted for presentation at the CFD4NRS-6, MIT, Cambridge, MA, USA, September 13-15.

Liang, R. et al., (2015) "Status Report on Hydrogen Management and Related Computer Codes“, NEA/CSNI/R(2014)8, 
Lopez-Alonso E., Papini D., and Jimenez G. (2017) “Hydrogen Distribution and Passive Autocatalytic Recombiner (PAR) Mitigation in a PWR-KWU Containment Type”, Annals of Nuclear Energy, 109, pp. 600-611.

Mahaffy, J., et al. (2015). Best Practice Guidelines for the Use of CFD in Nuclear Reactor Safety Applications - Revision” NEA/CSNI/R(2014)11.

Noutsopoulos, G.C. and Yannopoulos, P.C. (1989) “Axial Dilution in Obstructed Round Buoyant Jet”, Journal of Hydraulic Engineering, 115(1), pp. 71-81.

Nishimura, T., Hoshi, H. and Hotta, A. (2015) "Current Research and Development Activities on Fission Products and Hydrogen Risk after the Accident at Fukushima Daiichi Nuclear Power Station”, Nucl. Eng. Technol., 47, 1-10

OECD/NEA HYMERES-2 project (2017-2021).

OECD/NEA THAI Project (2010) "Hydrogen and Fission Product Issues Relevant for Containment Safety Assessment under Severe Accident Conditions” Final Report, 22 June 2010, Report NEA/CSNI/R(2010)3.

Paladino, D. and Dreier, J. (2012) “PANDA a Multi Purposes Integral Test Facility”, Science and Technology of Nuclear Installations, 2012, Article ID 239319, doi:10.1155/2012/239319.

Paladino, D., Mignot, G., Kapulla, R., Zboray, R., Andreani, M., Tkatschenko, I., Studer, E., and Brinster, J. (2013) “OECD/SETH-2 Project: PANDA and MISTRA Experiments addressing Key Safety Issues for Water Reactor Containment”, Proceedings of the $15^{\text {th }}$ International Topical Meeting on Nuclear Reactor Thermal-hydraulics (NURETH-15), paper 106, Pisa, Italy, 12-17 May 12-17, 2013.

Paladino, D., Andreani, M., Guentay, S., Mignot, G., Kapulla, R., Paranjape, S., Sharabi, M., Kisselev, A.,Yudina, T., Filippov, Kamnev, M., Khizbullin, A., Tyurikov, O., Liang, Z., Daniele Abdo, D., Brinster, J., Dabbene, F., Kelm, S., Klauck, M., Götz, L., Gehr, R., Malet, J., Bentaib, A., Bleyer, A., Lemaitre, P., Porcheron, E., Benz, S., Jordan, T., Xu, Z., Boyd, C., Siccama, A., Visser, D. (2016) "Outcomes from the EURATOM-ROSATOM ERCOSAM SAMARA projects on 
containment thermal-hydraulics for severe accident management”, Nuclear Eng. and Design, 308 , 103-114.

Paranjape, S., Kapulla, R., Mignot, G. and Paladino D. (2018) "Gas Redistribution Caused by Interacting Heat Sources in the Presence of a Vertical Condenser,” $12^{\text {th }}$ International Topical Meeting on Nuclear Reactor Thermal-Hydraulics, Operation and Safety (NUTHOS-12), Qingdao, China, October 14-18, 2018.

Sarikurt, F.S. and Hassan, Y.A. (2017) “Large Eddy Simulations of Erosion of a Stratified Layer by a Buoyant Jet”, Int. J. of Heat and Mass Transfer, Vol. 112, p. 354-365.

Schramm, B., Stewering, J., and Sonnenkalb M. (2017) "Einsatz von CFD-Codes für die Simulation von unfalltypischen Phänomenen im Sicherheitseinschluss: Validierung und gezielte

Modellerweiterung”, Anschlussbericht RS1526, 2017 GRS-472, ISBN 978-3-946607-55-7.

Schwarz, S., Fischer, K., Bentaib, A., Burkhardt, J., Lee, J.-J., Duspiva, J., Visser, D., Kyttala, J., Royl, P., Kim, J., Kostka, P. and Liang, R. (2011) "Benchmark on Hydrogen Distribution in a Containment based on the OECD-NEA THAI HM-2 Experiment”, Nucl. Technol., 175(3), 594-603. Smith B.L. (2009) "Identification and Prioritization of Generic Nuclear Safety Problems Requiring CFD Analysis”, Proc. 17 $7^{\text {th }}$ Int. Conf. on Nuclear Engineering (ICONE-17), Paper 75482, Brussels, Belgium, July12-16, 2009.

Studer, E., Brinster, J., Tkatschenko, I., Mignot, G., Paladino, D., and Andreani, M. (2012) "Interaction of a light gas stratified layer with an air jet coming from below: Large scale experiments and scaling issues”, Nucl. Eng. Design, 253, 406-412.

Visser, D.C., Houkema, M., Siccama, N.B., and Komen, E.M.J. (2012) "Validation of a FLUENT CFD model for hydrogen distribution in a containment”, Nuclear Engineering and Design, 245, 161$\underline{171 .}$
Formatted: Font: (Default) Times New Roman, Italic Formatted: Font: (Default) Times New Roman Formatted: Font: (Default) Times New Roman, Bold Formatted: Font: (Default) Times New Roman 


\section{LIST OF TABLES}

Table 1: Summary of submissions for the blind benchmark

Table 2: Overview of contributions for Step 1 (Exp. HP1_6_0) of the open benchmark

Table 3: Overview of contributions for Step 2 (Exp. HP1_7_0) of the open benchmark

Table 4: Overview of contributions for Step 3 (Exp. HP1 6 0) of the open benchmark

Table 5: Overview of simulations performed for Step 5 (Exp. ST1_2_2) of the open benchmark

Table 6: Overview of contributions for Step 4 (Exp. HP1_6_2) of the open benchmark 
Table 1: Summary of submissions for the blind benchmark

\begin{tabular}{|c|c|c|c|c|}
\hline & \multicolumn{3}{|l|}{$\mathrm{CM}$} & \multirow{2}{*}{$\begin{array}{l}\text { BEM } \\
\text { Differences from CM }\end{array}$} \\
\hline $\begin{array}{l}\text { Organi- } \\
\text { sation } \\
\text { Contri- } \\
\text { bution* }\end{array}$ & Code & $\frac{\frac{\text { Numb }}{\text { er of }}}{\frac{\text { cells }}{x 10^{3}}}$ & $\begin{array}{l}\text { Main } \\
\text { deviations } \\
\text { from } \\
\text { specifications }\end{array}$ & \\
\hline AERB & FLUENT 16 & 163 & - & RNG k- $\varepsilon$ \\
\hline BARC & $\begin{array}{l}\text { CFD- } \\
\mathrm{ACE}+\end{array}$ & 164 & $\begin{array}{l}\text { Injected steam } \\
\text { temperature }\end{array}$ & RNG k- $\varepsilon$ \\
\hline CIEMAT & FLUENT 15 & 135 & - & $\begin{array}{l}\text { radiative heat transfer (Discrete } \\
\text { Ordinates Method) included }\end{array}$ \\
\hline FZJ & CFX-15 & 565 & $\begin{array}{l}\text { Constant } \\
\text { pressure }\end{array}$ & $\begin{array}{l}\text { SST, condensation and radiative heat } \\
\text { transfer included (Monte Carlo Method). } \\
\text { Initial gas and wall } \mathrm{T} \text { distribution. }\end{array}$ \\
\hline GRS & CFX-15 & 1412 & - & $\begin{array}{l}\text { Slightly refined mesh ( } 1.7 \text { million cells), } \\
\text { SST, radiative heat transfer (Discrete } \\
\text { Transfer Model included }\end{array}$ \\
\hline $\begin{array}{l}\text { IBRAE } \\
\text { IBRAE }\end{array}$ & CFX-12 & 110 & $\begin{array}{l}\text { No man-hole, } \\
\text { injected steam } \\
\text { temperature }\end{array}$ & Different code (CABARET) \\
\hline $\begin{array}{l}\text { IBRAE } \\
\text { IBRAE2 }\end{array}$ & $\begin{array}{l}\text { FLUENT } \\
14.5 .7\end{array}$ & 355 & $\begin{array}{l}\text { No man-hole, } \\
\text { plate } \\
\text { thickness }=0\end{array}$ & RSM \\
\hline KAERI & $\begin{array}{l}\text { OpenFOAM } \\
2.3 .1\end{array}$ & 158 & $\begin{array}{l}\text { Square pipe } \\
\text { and plate }\end{array}$ & \\
\hline $\mathrm{S} / \mathrm{NRA} / \mathrm{R}$ & FLUENT 15 & 655 & - & $\begin{array}{l}\text { SST, pre-conditioning phase }(\mathrm{He} \\
\text { injection) simulated }\end{array}$ \\
\hline $\begin{array}{l}\text { PSI } \\
\text { PSIF }\end{array}$ & FLUENT 15 & 560 & $\begin{array}{l}\text { Half vessels, } \\
\text { no man-hole }\end{array}$ & \\
\hline $\begin{array}{l}\text { PSI } \\
\text { PSIG }\end{array}$ & GOTHIC 8.1 & 20 & Square plate & $\begin{array}{l}\text { Initial gas and wall } \mathrm{T} \text { distribution. } \\
\text { Enhanced HTC at the walls }\end{array}$ \\
\hline SPICRI & CFX-13 & 1284 & $\begin{array}{l}\text { Initial } \mathrm{T} \text { and } \\
\mathrm{X}_{\mathrm{He}} \text {. No wall } \\
\text { heat capacity }\end{array}$ & SST \\
\hline VTT & FLUENT 16 & 2872 & & \\
\hline
\end{tabular}

*for multiple contributions from the same organisation 
Table 2: Overview of contributions for Step 1 (Exp. HP1_6_0) of the open benchmark

\begin{tabular}{|c|c|c|c|c|c|}
\hline $\begin{array}{l}\text { Organi- } \\
\text { sation }\end{array}$ & Code & $\begin{array}{l}\text { Number of } \\
\text { cGells } \times 10^{3} \\
\text { (Nr. Cells for } \\
\text { Blind } \\
\text { simulations) } \\
\text { (U=unstructured } \\
\text { mesh) }\end{array}$ & $\begin{array}{l}\text { Reported } \\
\text { deviations from } \\
\text { specifications }\end{array}$ & $\begin{array}{l}\text { Size of cells } \\
\text { below } \\
\text { obstruction } \\
\text { (m) }\end{array}$ & $\begin{array}{l}\text { Equivalent } \\
\text { CPU time } \\
\text { on one } \\
\text { core } \\
\text { (h) }\end{array}$ \\
\hline AERB & $\begin{array}{l}\text { FLUENT } \\
\text { V.16 }\end{array}$ & $\begin{array}{l}240 \\
(163)\end{array}$ & $\begin{array}{l}\text { Constant } \\
\text { Pressure B.C. }\end{array}$ & $\begin{array}{l}0.03 \\
y+=40-125\end{array}$ & 1500 \\
\hline BARC & $\begin{array}{l}\text { CFD-ACE+ } \\
\text { Version } \\
2011\end{array}$ & $\begin{array}{l}690(\mathrm{U}) \\
(164)\end{array}$ & $\begin{array}{l}\text { Constant } \\
\text { Pressure B.C. }\end{array}$ & $\begin{array}{l}0.03 \\
y^{+=}=30-50\end{array}$ & 848 \\
\hline CIEMAT & $\begin{array}{l}\text { FLUENT } \\
16.2\end{array}$ & $\begin{array}{l}256(U) \\
(135)\end{array}$ & $\begin{array}{l}350 \mathrm{~s} \text { transient } \\
\text { time }\end{array}$ & $\begin{array}{l}(\mathrm{V}=9.4 \mathrm{e}-7 \\
\left.\mathrm{m}^{3}\right) \\
3 \sqrt{ } \mathrm{V} \sim 0.01\end{array}$ & 24 \\
\hline FZJ & CFX-17 & $\begin{array}{l}570 \\
(565)\end{array}$ & - & $\begin{array}{l}0.005 \\
y^{+\sim 30}\end{array}$ & 1606 \\
\hline KAERI & $\begin{array}{l}\text { OpenFOAM } \\
2.4 \mathrm{X}\end{array}$ & $\begin{array}{l}260 \\
(158)\end{array}$ & - & 0.04 & 375 \\
\hline S/NRA/R & $\begin{array}{l}\text { FLUENT } \\
\text { V15.0 }\end{array}$ & $\begin{array}{l}843(\mathrm{U}) \\
(655)\end{array}$ & - & 0.0036 & 696 \\
\hline PSI & $\begin{array}{l}\text { GOTHIC } \\
8.1\end{array}$ & $\begin{array}{l}41 \\
(20)\end{array}$ & - & $\begin{array}{l}0.01 \\
\mathrm{y}^{+\sim 17-38}\end{array}$ & 768 \\
\hline VTT & $\begin{array}{l}\text { FLUENT } \\
\text { V16.0 }\end{array}$ & $\begin{array}{l}2214 \\
(2872)\end{array}$ & $\begin{array}{l}\text { Enhanced wall } \\
\text { treatment }\end{array}$ & 0.0125 & 2500 \\
\hline
\end{tabular}


Table 3: Overview of contributions for Step 2 (Exp. HP1_7_0) of the open benchmark

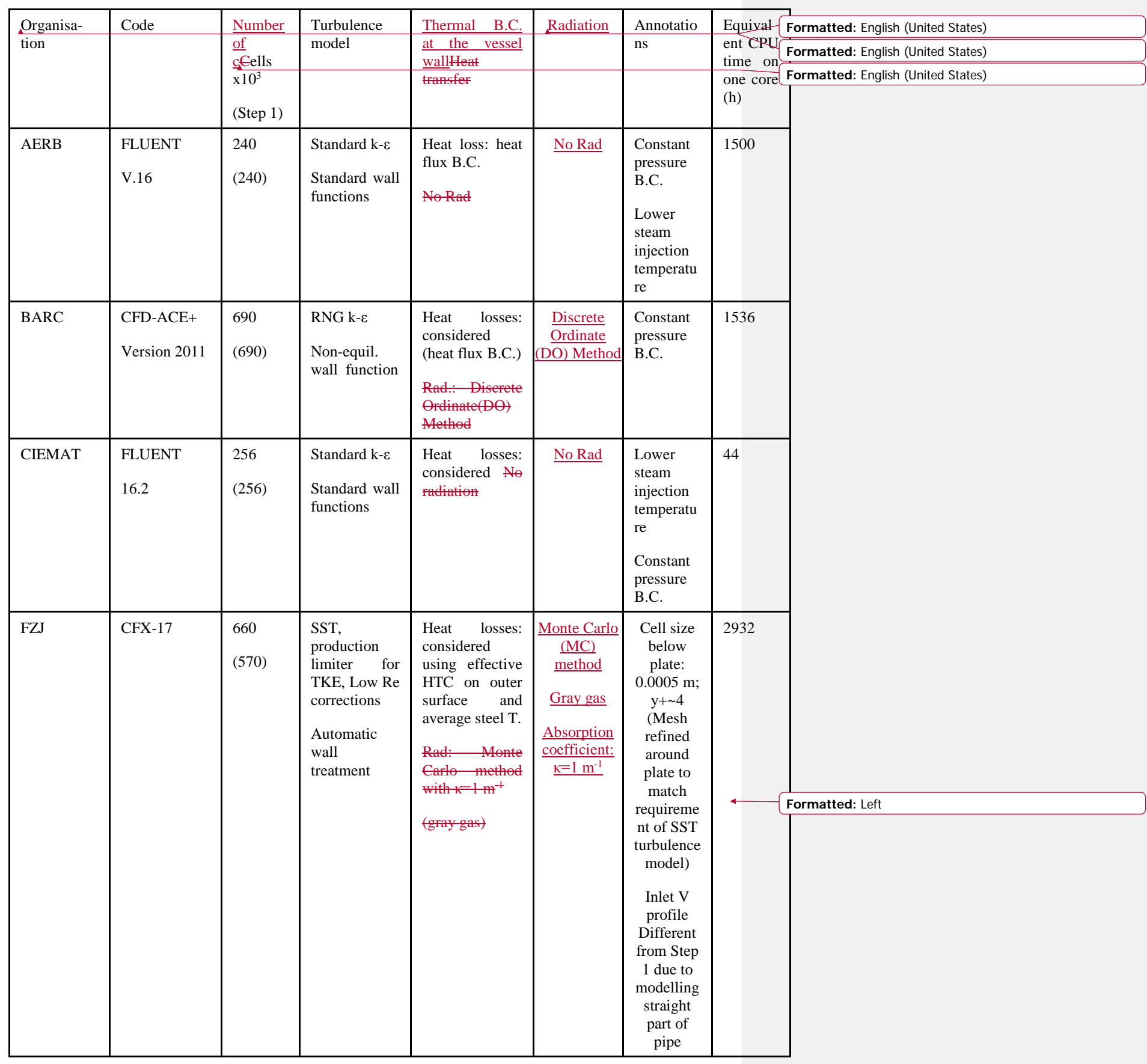


1

2

\begin{tabular}{|c|c|c|c|c|c|c|c|}
\hline KAERI & $\begin{array}{l}\text { OpenFOAM } \\
2.4 \mathrm{X}\end{array}$ & $\begin{array}{l}260 \\
(260)\end{array}$ & $\begin{array}{l}\text { Standard k- } \varepsilon \\
\text { Standard wall } \\
\text { functions }\end{array}$ & $\begin{array}{l}\text { No Rad. } \\
\text { No heat losses. } \\
\text { Fix wall } \\
\text { temperature }\end{array}$ & No Rad. & $\begin{array}{l}\text { Flat } \mathrm{V} \\
\text { profile at } \\
\text { pipe exit }\end{array}$ & 672 \\
\hline S/NRA/R & $\begin{array}{l}\text { FLUENT } \\
\text { V15.0 }\end{array}$ & 843 & $\begin{array}{l}\text { k- } \omega \quad \text { SST, } \\
\text { Kato- } \\
\text { Launder, } \\
\text { Low Re } \\
\text { corrections }\end{array}$ & $\begin{array}{l}\text { No Rad. } \\
\text { Constant Wall } \\
\text { temp. }\end{array}$ & No Rad. & $\begin{array}{l}\text { Constant } \\
\text { Pressure } \\
\text { B.C. } \\
\text { Inlet V } \\
\text { profile }\end{array}$ & 928 \\
\hline PSI & $\begin{array}{l}\text { GOTHIC } \\
8.2 \mathrm{a}\end{array}$ & $\begin{array}{l}42 \\
(41) \\
\text { Injec- } \\
\text { tion } \\
\text { pipe } \\
\text { model- } \\
\text { led }\end{array}$ & $\begin{array}{l}\text { Standard k- } \varepsilon \\
\text { Standard wall } \\
\text { functions }\end{array}$ & $\begin{array}{l}\text { Heat losses: } \\
\text { heat flux B.C. } \\
\text { No Rad. }\end{array}$ & No Rad. & $\begin{array}{l}\text { Inlet } \mathrm{V} \\
\text { and } \mathrm{T} \\
\text { profiles } \\
\text { obtained } \\
\text { modelling } \\
\text { the } \\
\text { injection } \\
\text { pipe } \\
\text { (tuning } \\
\text { the vessel } \\
\text { inlet T) } \\
\text { New } \\
\text { version of } \\
\text { the code } \\
\text { (8.2) }\end{array}$ & 444 \\
\hline VTT & $\begin{array}{l}\text { FLUENT } \\
\text { V16.0 }\end{array}$ & $\begin{array}{l}2388 \\
(2872) \\
\text { Finer } \\
\text { than for } \\
\text { Step 1 }\end{array}$ & $\begin{array}{l}\text { k- } \omega \text { SST, } \\
\text { Production } \\
\text { Kato- } \\
\text { Launder, } \\
\text { Production } \\
\text { Limiter, } \\
\text { low-Re } \\
\text { correction } \\
\\
\\
\text { Near-wall } \\
\text { treatment }\end{array}$ & $\begin{array}{l}\text { No Rad } \\
\text { Constant Wall } \\
\text { temp. }\end{array}$ & No Rad & $\begin{array}{l}\text { Grid } \\
\text { includes } \\
\text { injection } \\
\text { pipe with } \\
\text { heat } \\
\text { structures. } \\
\text { Experime } \\
\text { ntal Mass } \\
\text { inflow } \\
\text { and } \\
\text { temperatu } \\
\text { re defined } \\
\text { into the } \\
\text { inlet pipe. }\end{array}$ & N/A \\
\hline
\end{tabular}


1

2

3

4

5

6

Table 4: Overview of contributions for Step 3 (Exp. HP1_6_0) of the open benchmark

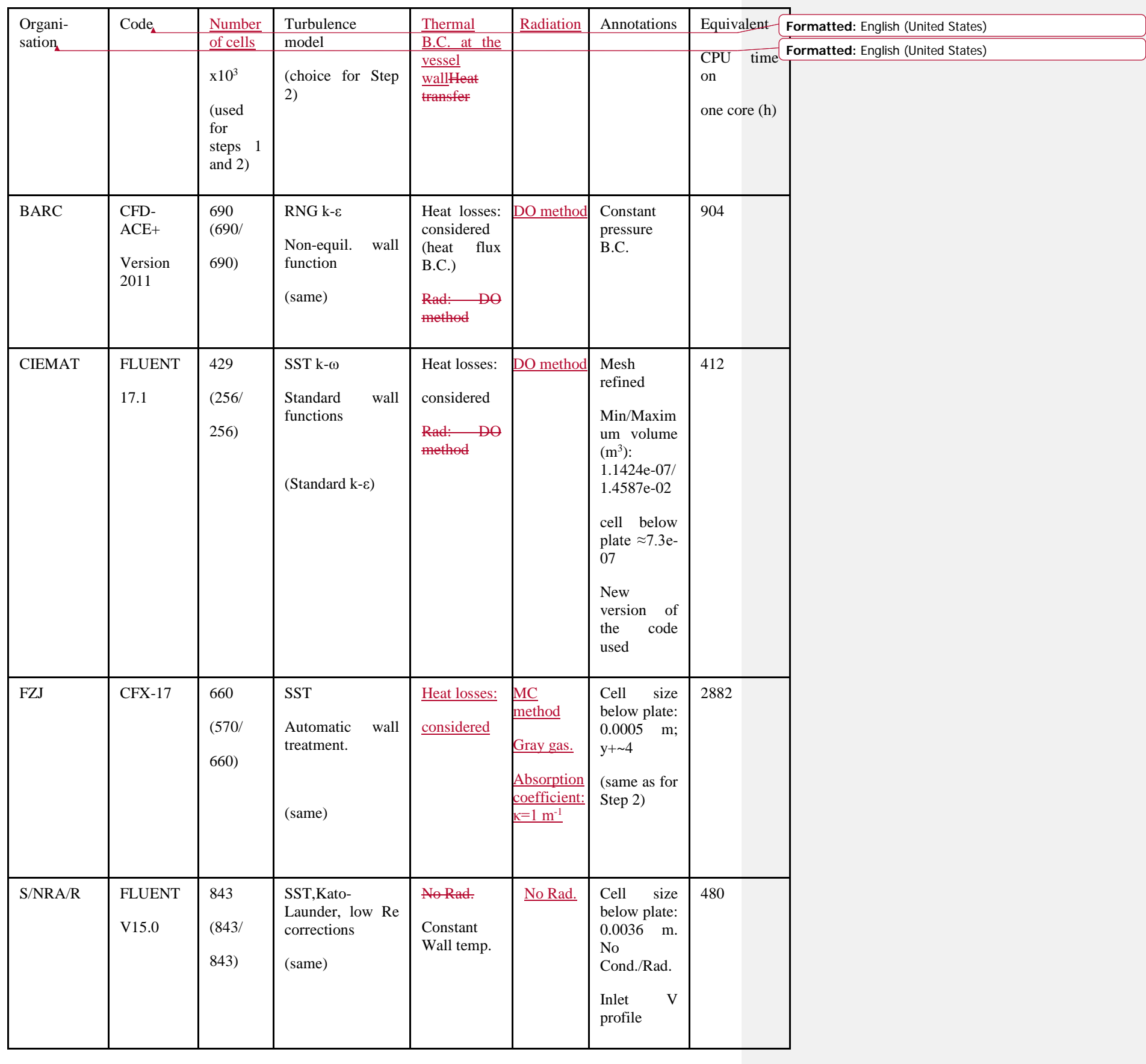


1

2 
Table 5: Overview of simulations performed for Step 5 (Exp. ST1 2 2) of the open benchmark

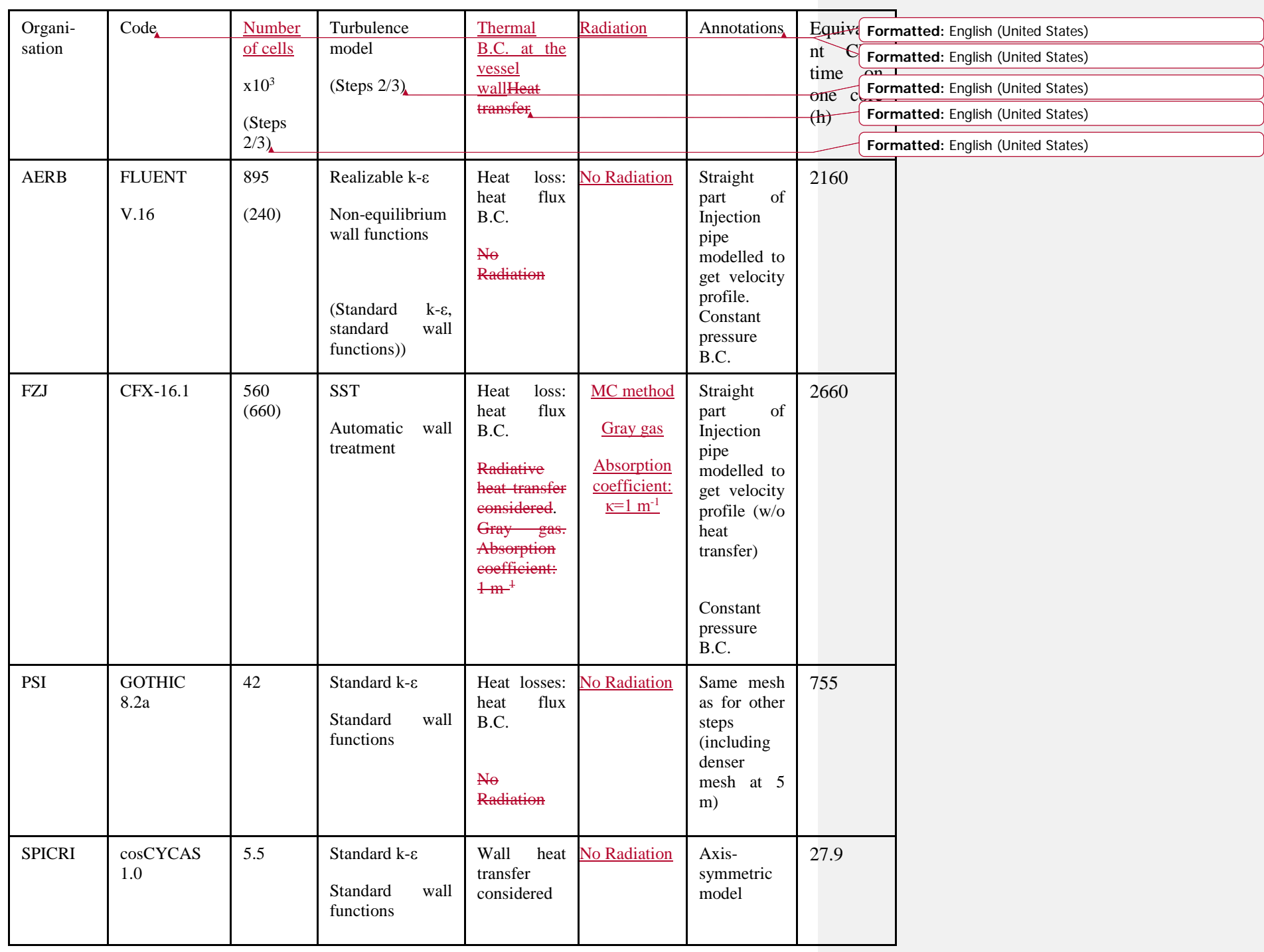


Table 6: Overview of contributions for Step 4 (Exp. HP1_6_2) of the open benchmark

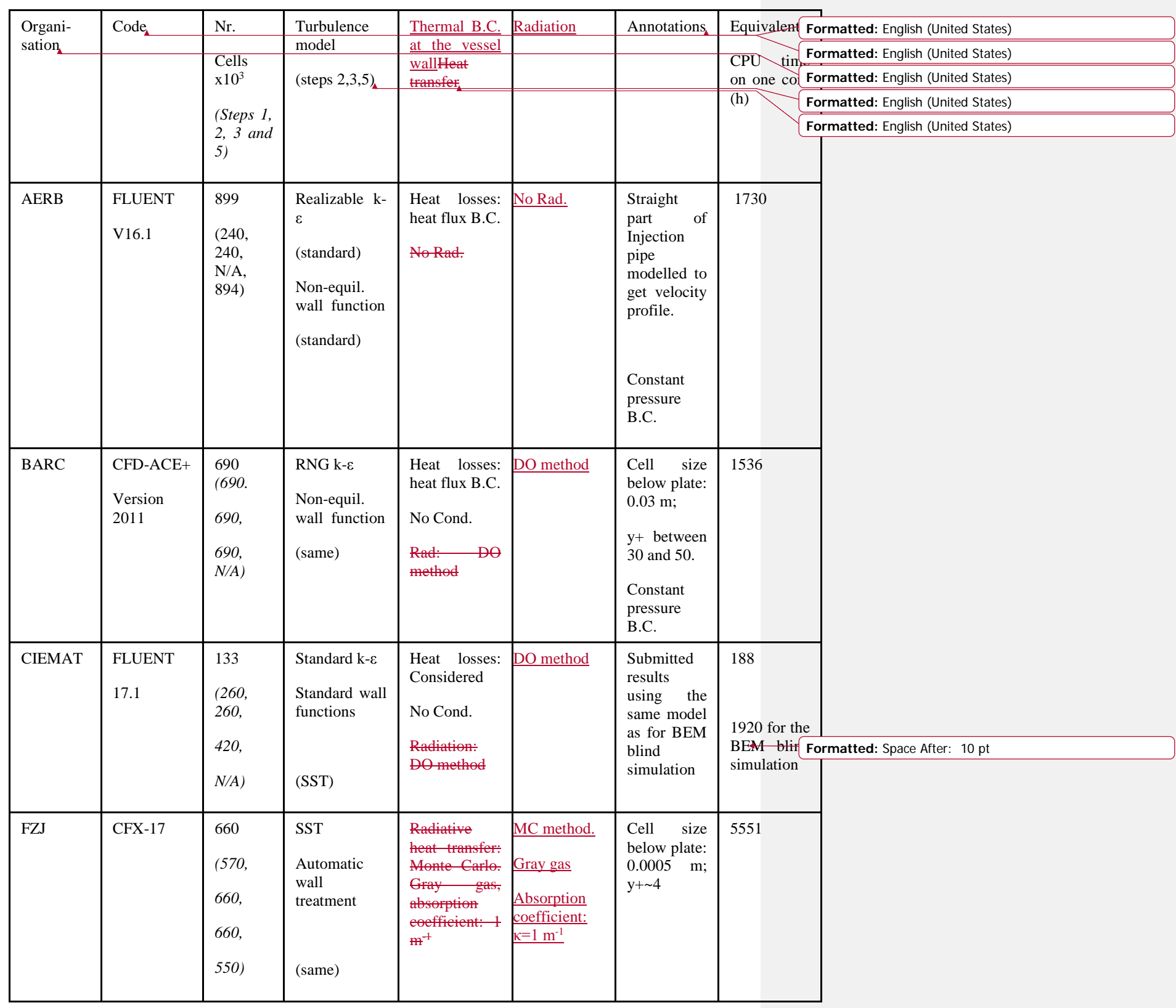


1

2

4

5

\begin{tabular}{|c|c|c|c|c|c|c|c|c|}
\hline GRS & CFX-17 & 7000 & $\begin{array}{l}\text { SST, } \\
\text { Including } \\
\text { buoyancy } \\
\text { turbulence } \\
\text { terms } \\
\text { automatic } \\
\text { wall } \\
\text { treatment }\end{array}$ & $\begin{array}{l}\text { Radiative } \\
\text { heat transfer: } \\
\text { DTM (64 } \\
\text { rays), gray } \\
\text { gas, } \\
\text { absorption } \\
\text { coefficient } ~ \\
\mathrm{~m}^{-1} \text { (from } \\
\text { RS1500 } \\
\text { eorrelation) }\end{array}$ & 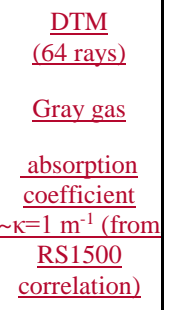 & $\begin{array}{l}\text { Straight part } \\
\text { of injection } \\
\text { pipe } \\
\text { modelled to } \\
\text { get velocity } \\
\text { profile }\end{array}$ & 17700 & \\
\hline \multirow[t]{7}{*}{$\mathrm{S} / \mathrm{NRA} / \mathrm{R}_{\mathrm{a}}$} & \multirow{7}{*}{$\begin{array}{l}\text { FLUENT }_{\Delta} \\
\text { V15.0 }\end{array}$} & \multirow{7}{*}{$\begin{array}{l}843 \\
(843, \\
843, \\
843, \\
N / A)\end{array}$} & \multirow{7}{*}{$\begin{array}{l}\text { SST, } \\
\text { Kato- } \\
\text { Launder, low } \\
\text { Re } \\
\text { corrections } \\
\text { no wall } \\
\text { treatment } \\
\text { (same) }\end{array}$} & \multirow{6}{*}{$\begin{array}{l}\text { No Cond. } \\
\text { No Rad. } \\
\text { Constant } \\
\text { Wall temp. }\end{array}$} & \multirow[t]{7}{*}{ No Rad. } & \multirow{7}{*}{$\begin{array}{l}\text { Cell size } \\
\text { below plate: } \\
0.0036 \mathrm{~m} \text {. } \\
\text { Constant } \mathrm{P} \\
\text { Inlet } \\
\text { profile }\end{array}$} & \multirow[t]{7}{*}{208} & Formatted: English (United States) \\
\hline & & & & & & & & Formatted: English (United States) \\
\hline & & & & & & & & Formatted: English (United States) \\
\hline & & & & & & & & Formatted: English (United States) \\
\hline & & & & & & & & \\
\hline & & & & & & & & \multirow[t]{3}{*}{ Formatted: English (United States) } \\
\hline & & & & & & & & \\
\hline PSI & $\begin{array}{l}\text { GOTHIC } \\
8.2\end{array}$ & $\begin{array}{l}42 \\
(41.42 \\
42,42 \\
)\end{array}$ & $\begin{array}{l}\text { Standard k- } \varepsilon \\
\text { Standard wall } \\
\text { functions } \\
\text { (same) }\end{array}$ & $\begin{array}{l}\text { Heat losses: } \\
\text { heat flux B.C. } \\
\text { Rad: not } \\
\text { eonsidered }\end{array}$ & No Rad. & $\begin{array}{l}\text { Contrarily to } \\
\text { BEM sE } \\
\text { simulation for } \\
\text { the blind } \\
\text { benchmark, } \\
\text { the low initial } \\
\text { values of the } \\
\text { wall } \\
\text { temperature } \\
\text { at the top of } \\
\text { the dome } \\
\text { were not used } \\
\text { for a large } \\
\text { part of the } \\
\text { dome, and } \\
\text { Standard } \\
\text { convective } \\
\text { HTC } \\
\text { correlation } \\
\text { were used (no } \\
\text { enhancement } \\
\text { factor) }\end{array}$ & 768 & \\
\hline
\end{tabular}


1

2 


\section{LIST OF FIGURES}

Figure 1: Cutaway drawing of the vessels (left), configuration for the experiment (middle), and initial helium concentration vertical profile in Vessel 1 (right).

Figure 2: Time-Evolutionhistories of helium concentrations (left) and gas temperatures (right) at various elevations along axis of Vessel 1.

Figure 3: Blind benchmark: Erosion progression (right) calculated with the CM and evolutiontime history (left) of gas temperature at position B20 (z=7500 mm), shown as an example. The dashed line shows how the erosion time at each elevation (e.g. at the elevation of position B20) is derived.

Figure 4: Blind benchmark: Helium concentration evolutiontime histories at elevation A (left) and B (right) calculated with the "Common Model” (CM)

Figure 5: Blind benchmark: Gas temperature horizontal profile at level $\mathrm{G}$ at $300 \mathrm{~s}$ (left) and at level $\mathrm{H}$ at $150 \mathrm{~s}$ (right) calculated with the CM. Square marks show the experimental data.

Figure 6: Blind benchmark: Erosion progression (left) and helium concentration evolutiontime history at Level B (right) calculated with the BEM.

Figure 7: (top) vertical section (plane $315^{\circ}-135^{\circ}$ ) and horizontal section of Vessel 1 showing the locations of the concentration and gas temperature measurements and the Field of View (FOV) for PIV measurements used for HP1_6_2 (Vessel 2 and IP are not shown); (bottom) position of the combined PIV window used for Tests HP1_X_0.

Figure 8: Experiments and models used in the five steps of the open benchmark.

Figure 98: Open benchmark, Step 1: Average experimental flow field (left) measured in Test HP1_6_0, and vertical velocity profiles used in the comparisons with calculated results (right).

Figure 109: Open benchmark, Step 1: Axial profile of the vertical velocity (left) and vertical profile of the maximum vertical velocity (right).

Figure 110: Open benchmark, Step 1: Measured distribution of Turbulent Kinetic Energy (left) and comparison between experimental and calculated axial profile

Figure 124: Open benchmark, Step 1: Difference of the maximum velocity in the two half planes (left) and position (Y2) of the full jet recovery (right).

Figure 1ㄹz: Open benchmark, Step 1: EvolutionTime history of the minimum vertical velocity (left) and elevation (Y1) of the top of the recirculation zone (right). 
Figure 143: Open benchmark, Step 1: Horizontal profiles of the vertical velocity at the elevation of the middle of the recirculation zone (left) and at an elevation in the flow developed region (right).

Figure 1며: Open benchmark, Step 1: Horizontal profiles of the TKE at the elevation of the middle of the recirculation zone (left) and at an elevation in the flow developed region (right).

Figure 165: Open benchmark, Step 2: Average experimental flow field (left) measured in Test HP1_7_0, and vertical velocity profiles used in the comparisons with calculated results (right).

Figure 176: Open benchmark, Step 2: Axial profile of the vertical velocity (left) and vertical profile of the maximum vertical velocity (right).

Figure 187: Open benchmark, Step 2: Temperature radial profile at $6.276 \mathrm{~m}$ (left) and axial temperature distribution (right).

Figure 198: Open benchmark, Step 3: Axial velocity and maximum velocity vertical profiles for Step 3, compared with results for Step 1.

Figure 2019: Open benchmark, Step 3: Axial temperature profile and horizontal profile at $6.3 \mathrm{~m}$.

Figure 21ㅂ: Open benchmark, Step 5: Configuration for SETH-2 test ST1_2_2, and important initial and boundary conditions of test ST1_2 and repetition test ST1_2_2 compared with those used for Test HP1_6_2.

Figure 221: Open benchmark, Step 5: Progression of erosion along the axis in test ST1_2_2, using gas temperature rise times.

Figure 23zz: Open benchmark, Step 5: Helium concentration evolutiontime histories at various elevations along the axis of Vessel 1.

Figure 243: Open benchmark, Step 5: Vertical velocity axial profile (left) and horizontal profile at $\mathrm{z}=5003 \mathrm{~mm}$ ( $1 \mathrm{~m}$ above pipe exit)

Figure 254: Open benchmark, Step 5: Axial temperature profile at two times.

Figure 265: Open benchmark, Step 5: horizontal profiles at $6.3 \mathrm{~m}$ at $150 \mathrm{~s}$ (before mixing) and $300 \mathrm{~s}$ (after mixing)

Figure 276: Open benchmark, Step 5: horizontal profiles at $6.93 \mathrm{~m}$ at $250 \mathrm{~s}$ (during mixing or immediately after) and $500 \mathrm{~s}$ (after mixing)

Figure 28: Open benchmark, Step 4: Calculated sequence of spatial distributions of helium concentrations at various times (contribution of VTT). 
Figure 29: Open benchmark, Step 4: Calculated sequence of spatial distributions of gas temperatures at various times (contribution by VTT).

Figure 3027: Open benchmark, Step 4: Erosion progression calculated in the open phase (left), compared with best estimate results contributed to the blind benchmark.

Figure 31z8: Open benchmark, Step 4: Helium concentration evolutiontime history at $\mathrm{z}=6.48 \mathrm{~m}$ calculated in the open phase (left), compared with best estimate results contributed to the blind benchmark.

Figure 32z9: Open benchmark, Step 4: Example of results obtained using the model for radiation or neglecting it: helium concentration evolutiontime histories calculated at $8 \mathrm{~m}$ (left, GRS, Schramm et al., 2017) and at $7.5 \mathrm{~m}$ (right, VTT, Huhtanen, 2018).

Figure 330: Open benchmark, Step 4: Vertical velocity averaged axial and horizontal profiles in the PIV window.

Figure 341: Open benchmark, Step 4: Axial gas temperature distributions at two times

Figure 3ㅍz: Open benchmark, Step 4: Gas temperature horizontal profiles at various elevations and two times.

Figure 36ㄱ: Open benchmark, Step 4: Gas temperature evolutiontime histories at two positions (left: on the axis; right: at $570 \mathrm{~mm}$ from the wall) at $6.5 \mathrm{~m}$.

Figure 374: Open benchmark, Step 4: Off-axis (r: $\pm 325 \mathrm{~mm}$ ) gas temperature evolutiontime histories at two positons below the initial bottom of the helium layer (z: 5301 and $4000 \mathrm{~mm}$, respectively) 


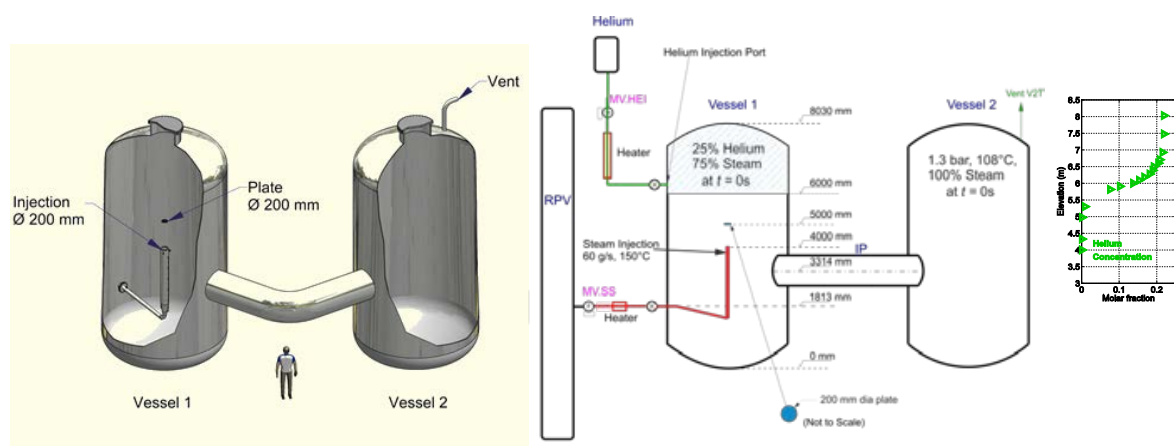

Figure 1: Cutaway drawing of the vessels (left), configuration for the experiment (middle), and initial helium concentration vertical profile in Vessel 1 (right).
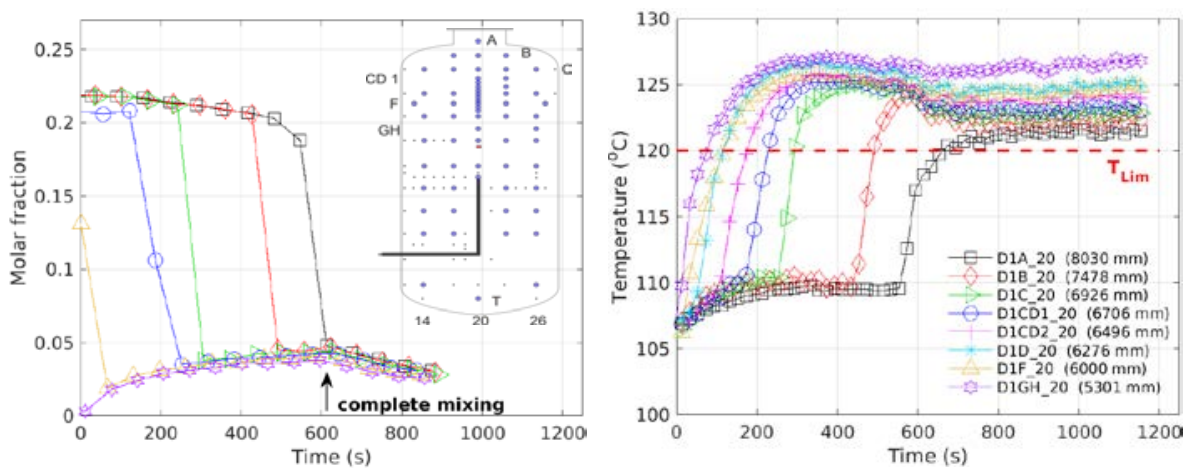

Figure 2: EvolutionTime histories of helium concentrations (left) and gas temperatures (right) at various elevations along axis of Vessel 1. 


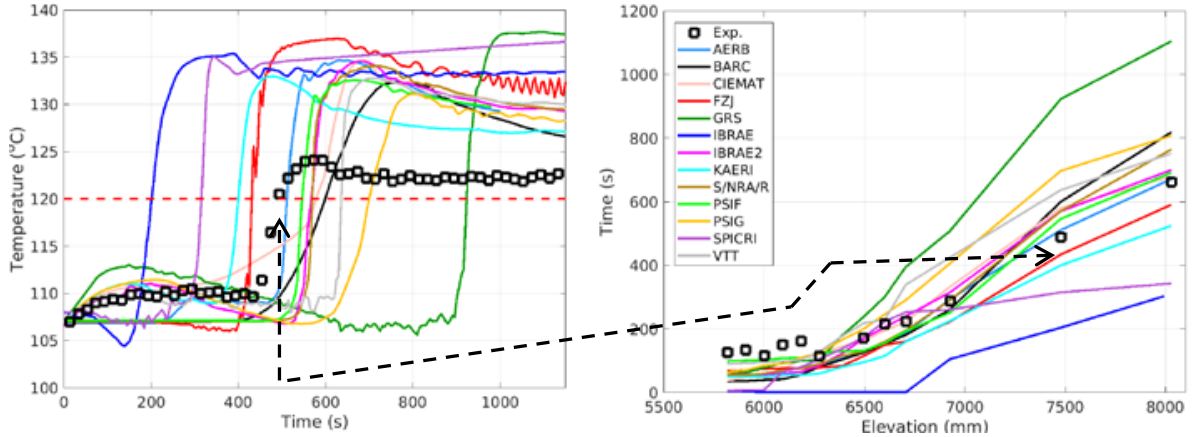

Figure 3: Blind benchmark: erosion progression (right) calculated with the CM and evolutiontime history (left) of gas temperature at position B20 (z=7500 mm), shown as an example. The dashed line shows how the erosion time at each elevation (e.g. at the elevation of position B20) is derived.
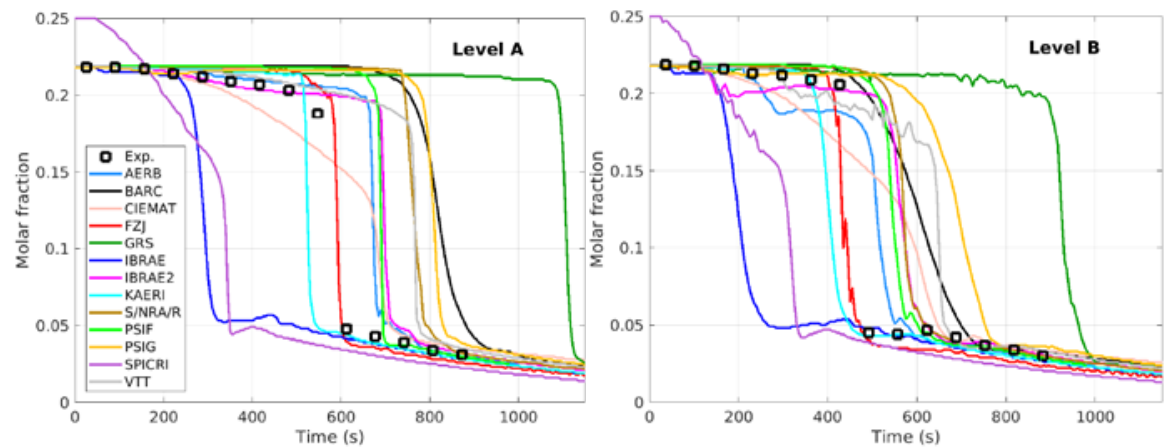

Figure 4: Blind benchmark: Helium concentration evolutiontime histories at elevation A (left) and B (right) calculated with the "Common Model” (CM).
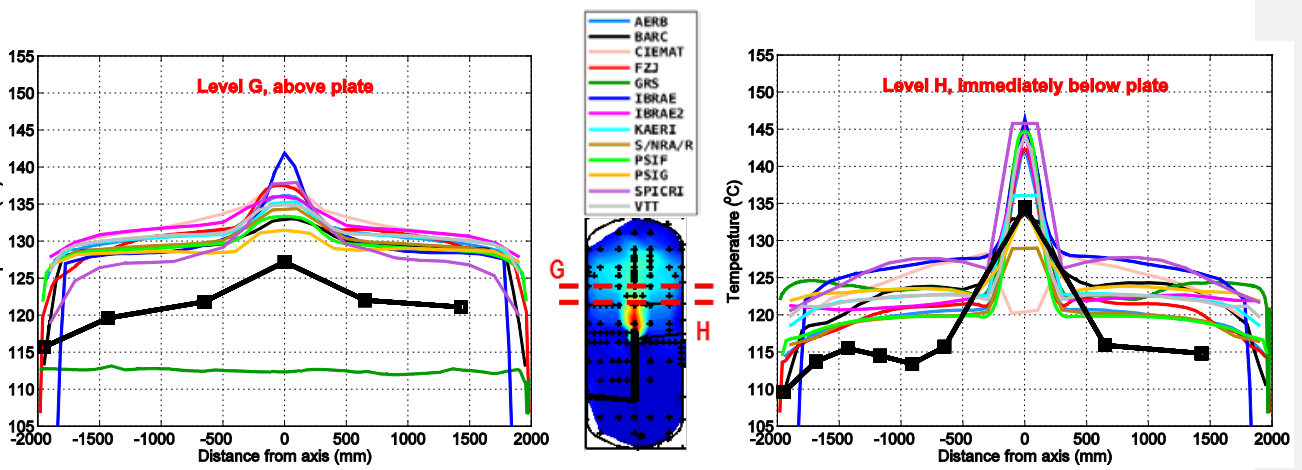
Figure 5: Blind benchmark: Gas temperature horizontal profile at level $\mathrm{G}$ at $300 \mathrm{~s}$ (left) and at level $\mathrm{H}$ at $150 \mathrm{~s}$ (right) calculated with the CM. Square marks show the experimental data.
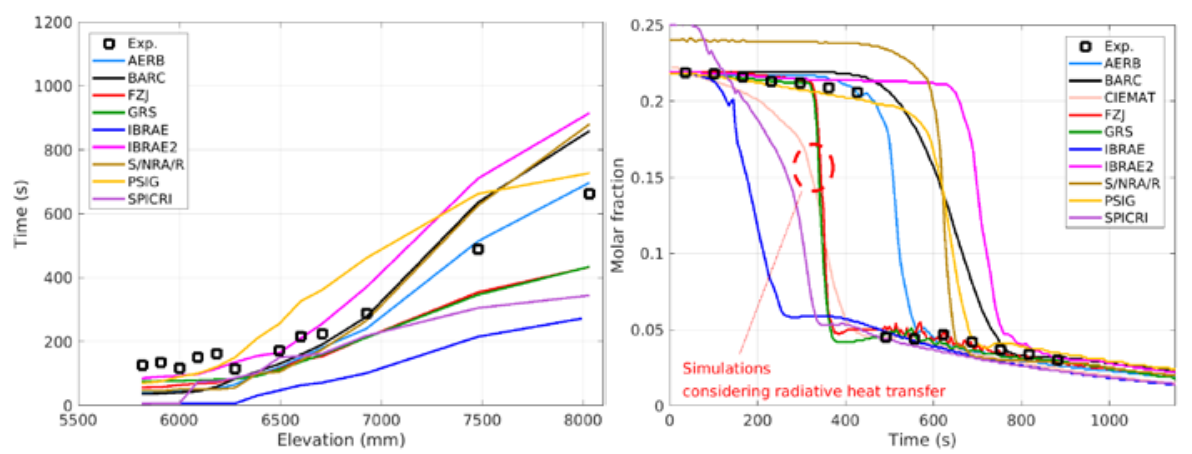

Figure 6: Blind benchmark: Erosion progression (left) and helium concentration evolutiontime history at Level B (right) calculated with the BEM.

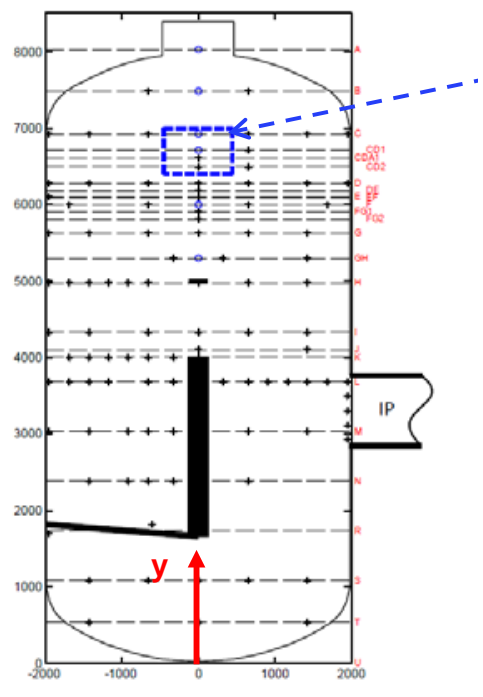

PIV Field of View (FOV)

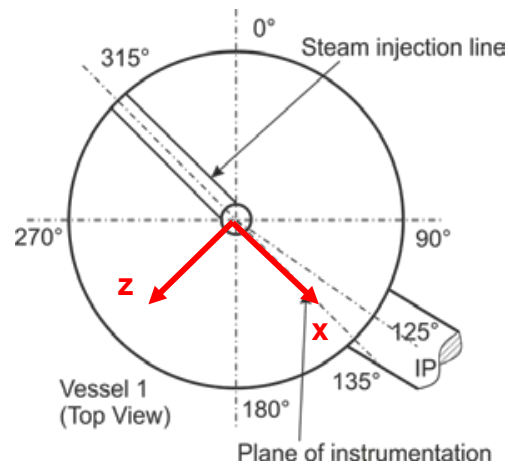




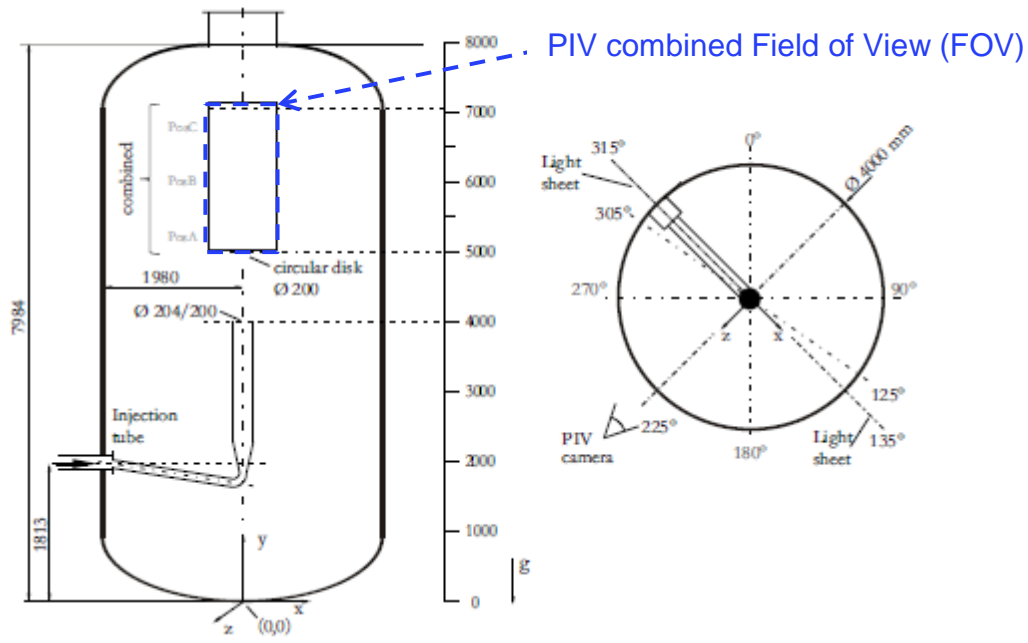

Figure 7: (top) vertical section (plane $315^{\circ}-135^{\circ}$ ) and horizontal section of Vessel 1 showing the locations of the concentration and gas temperature measurements and the Field of View (FOV) for PIV measurements used for HP1_6_2 (Vessel 2 and IP are not shown); (bottom) position of the combined PIV window used for Tests HP1_X_0.
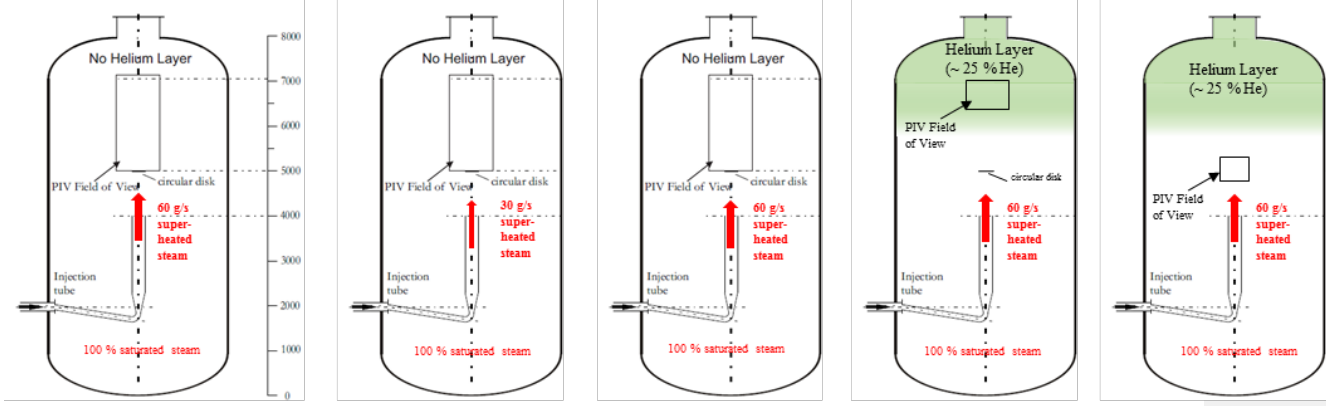

Step 1:

Step 2:

Step 3:

Step 4:

Exp: HP1 6 2 2 Step 5 (optional): Exp: $\mathrm{HP}_{-} 6$ Exp: HP1 $1{ }^{7}-0$ Exp: HP1 $6-0$ Model: $\mathrm{BEM}$

Figure 8: Experiments and models used in the five steps of the open benchmark. 

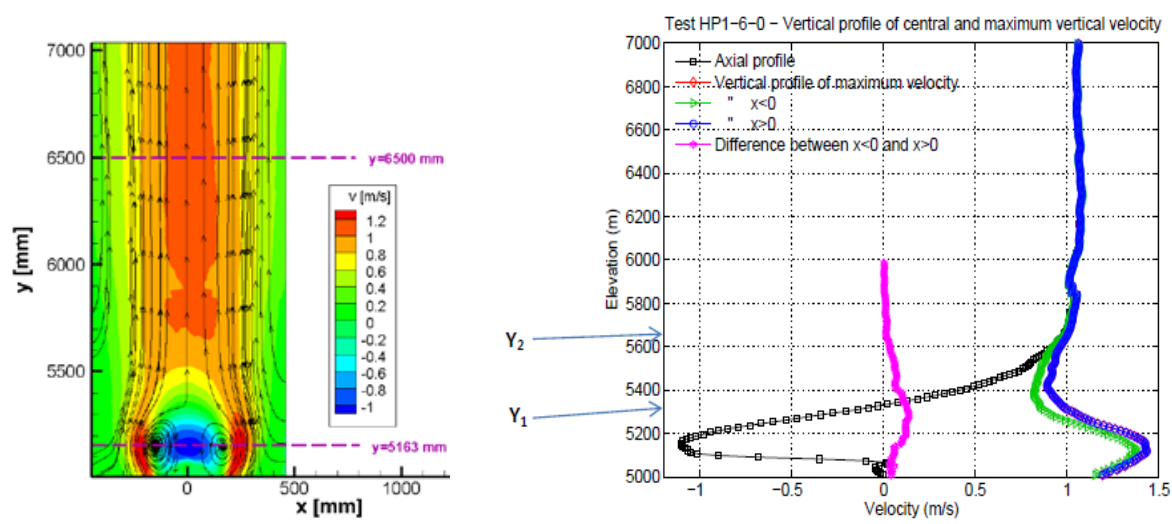

Figure 98: Open benchmark, Step 1: Average experimental flow field (left) measured in Test HP1_6_0, and vertical velocity profiles used in the comparisons with calculated results (right).
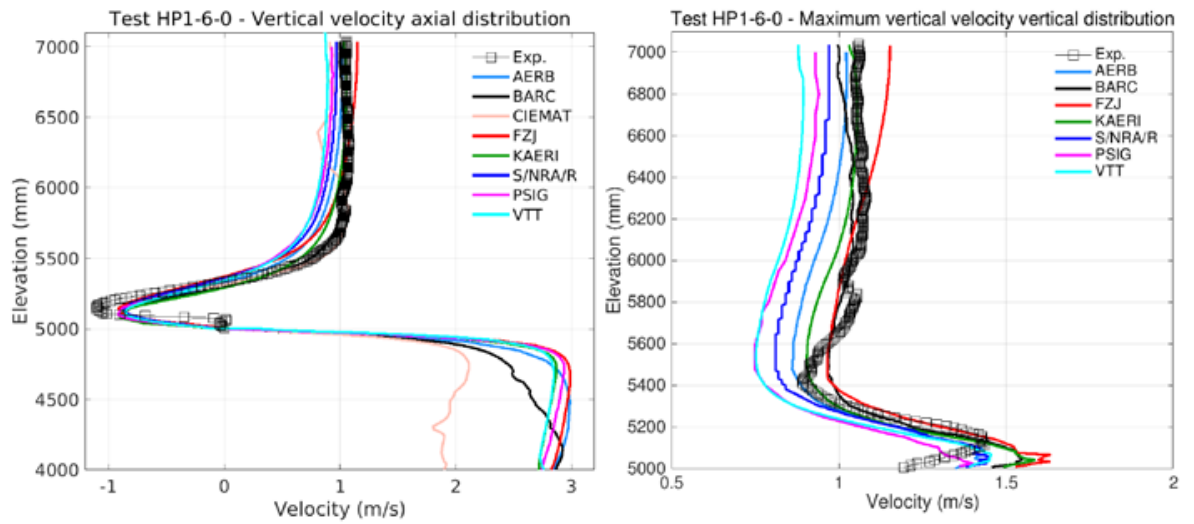

Figure 109: Open benchmark, Step 1: Axial profile of the vertical velocity (left) and vertical profile of the maximum vertical velocity (right). 

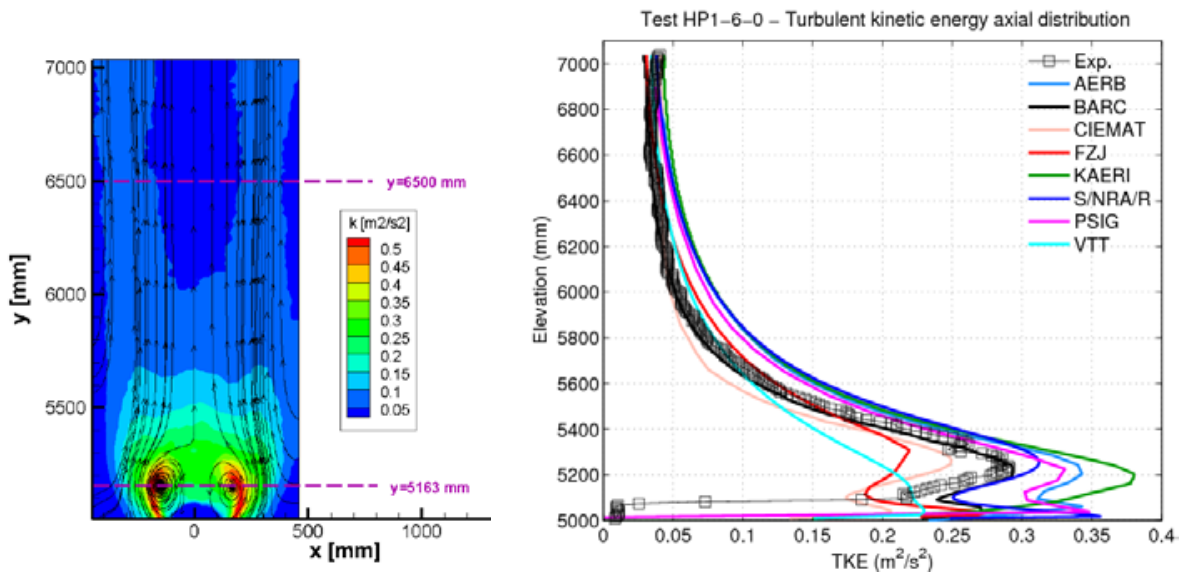

Figure 110: Open benchmark, Step 1: Measured distribution of Turbulent Kinetic Energy (left) and comparison between experimental and calculated axial profile.
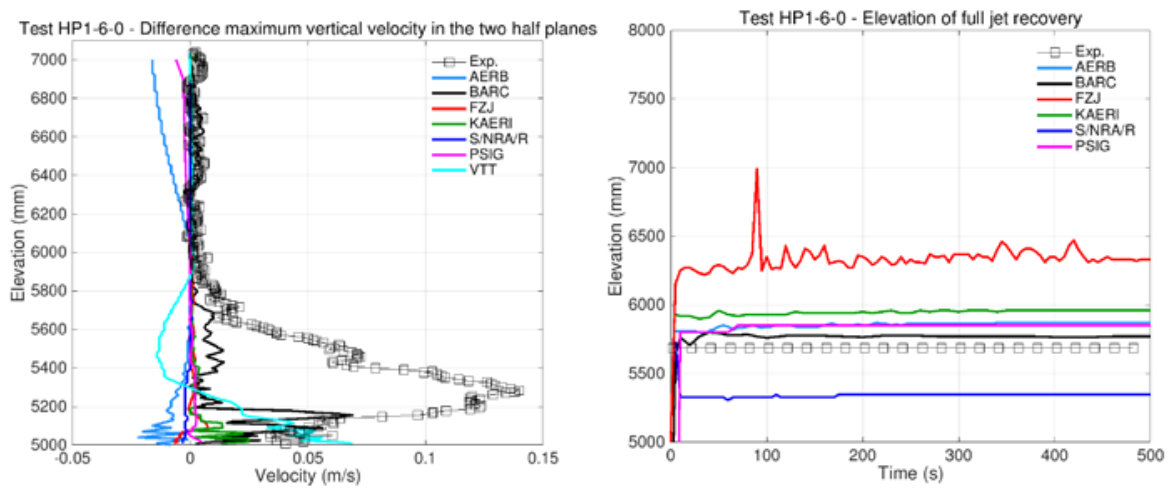

Figure 124: Open benchmark, Step 1: Difference of the maximum velocity in the two half planes (left) and position (Y2) of the full jet recovery (right). 

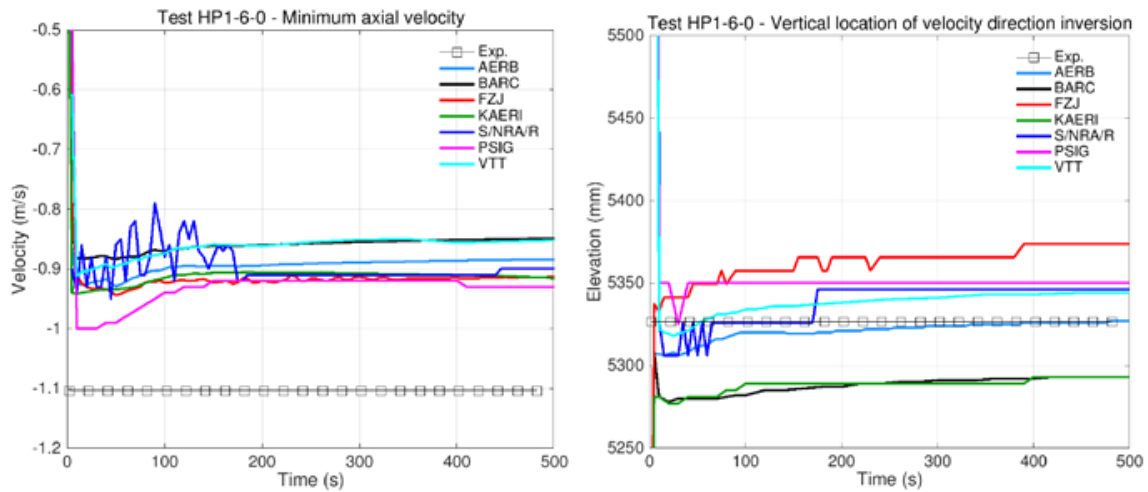

Figure 1ㄹz: Open benchmark, Step 1: EvolutionTime history of the minimum vertical velocity (left) and elevation (Y1) of the top of the recirculation zone (right).
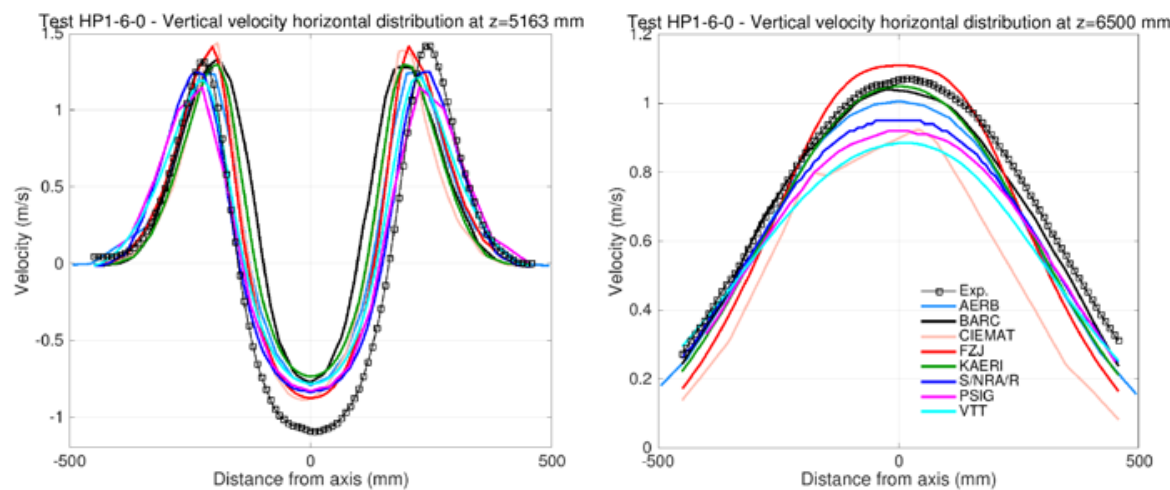

Figure 143: Open benchmark, Step 1: Horizontal profiles of the vertical velocity at the elevation of the middle of the recirculation zone (left) and at an elevation in the flow developed region (right). 

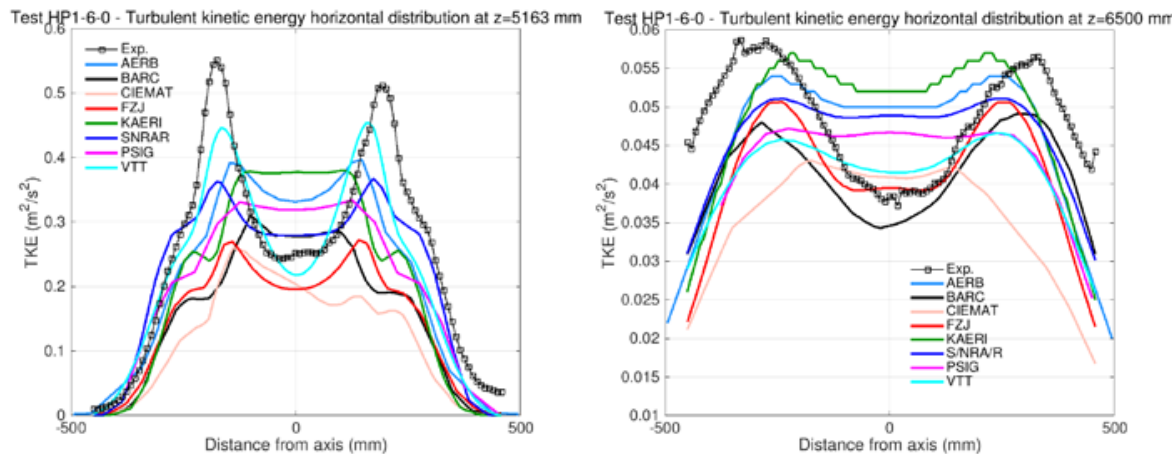

Figure 154: Open benchmark, Step 1: Horizontal profiles of the TKE at the elevation of the middle of the recirculation zone (left) and at an elevation in the flow developed region (right).
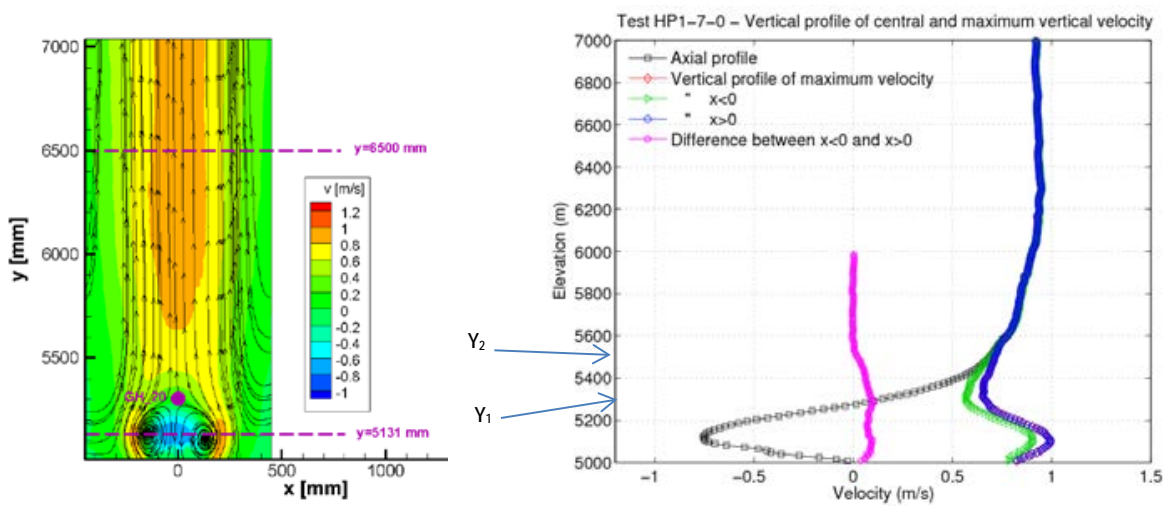

Figure 16ㄷ: Open benchmark, Step 2: Average experimental flow field (left) measured in Test HP1_7_0, and vertical velocity profiles used in the comparisons with calculated results (right). 

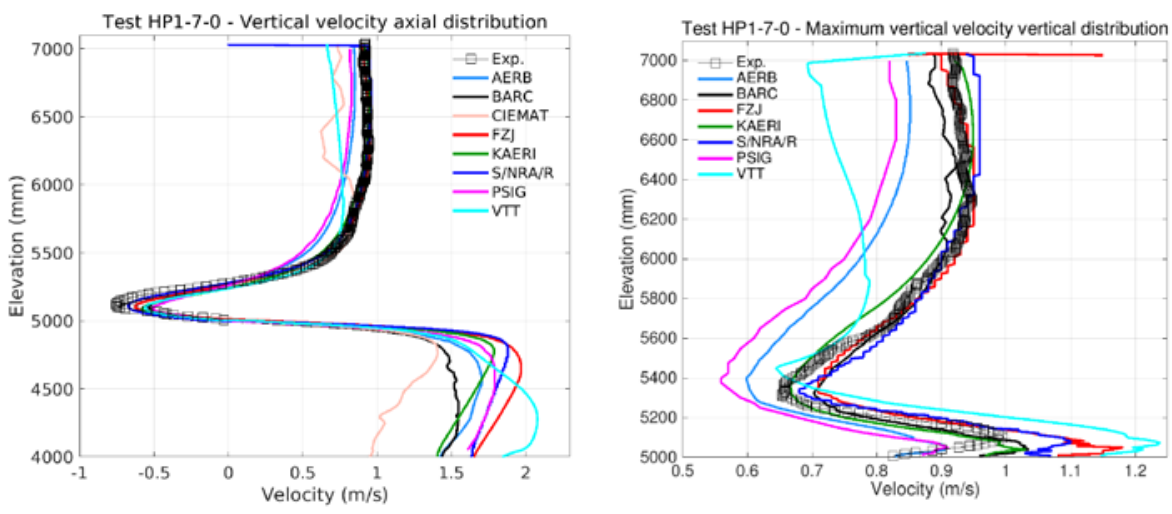

Figure 176: Open benchmark, Step 2: Axial profile of the vertical velocity (left) and vertical profile of the maximum vertical velocity (right).
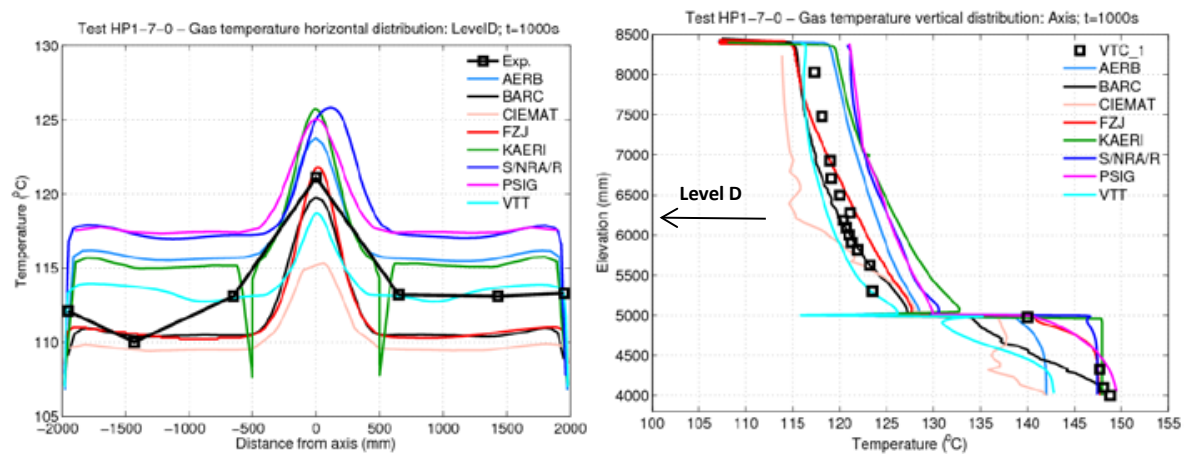

Figure 1ㅁ: Open benchmark, Step 2: Temperature radial profile at $6.276 \mathrm{~m}$ (left) and axial temperature distribution (right). 

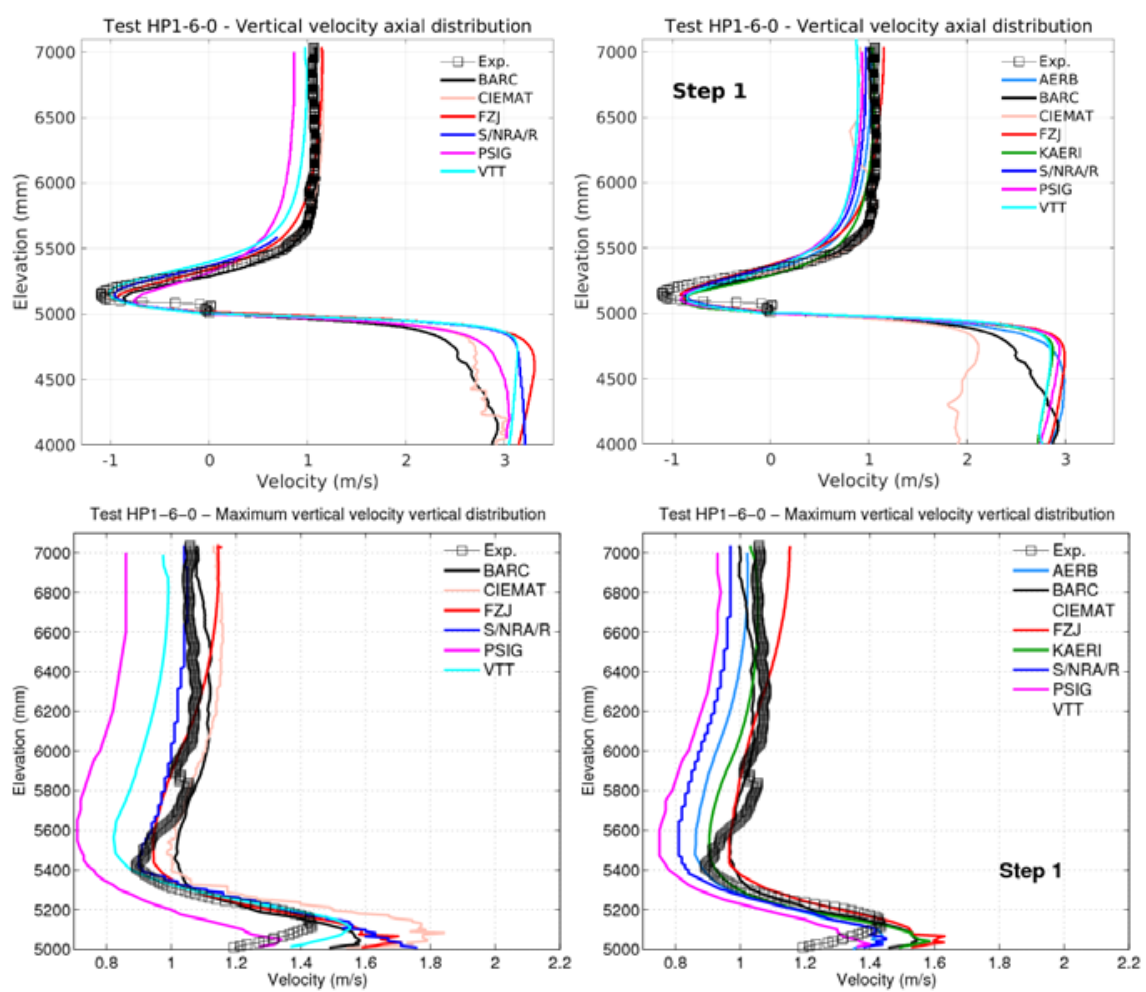

Figure 1998: Open benchmark, Step 3: Axial velocity and maximum velocity vertical profiles for Step 3, compared with results for Step 1 .
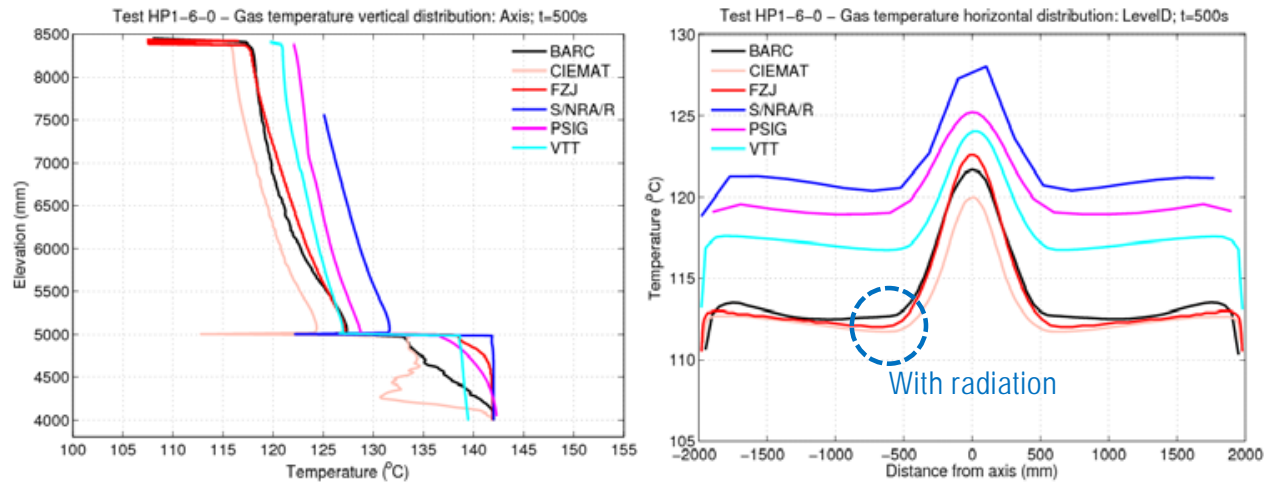

Figure 2019: Open benchmark, Step 3: Axial temperature profile and horizontal profile at $6.3 \mathrm{~m}$. 

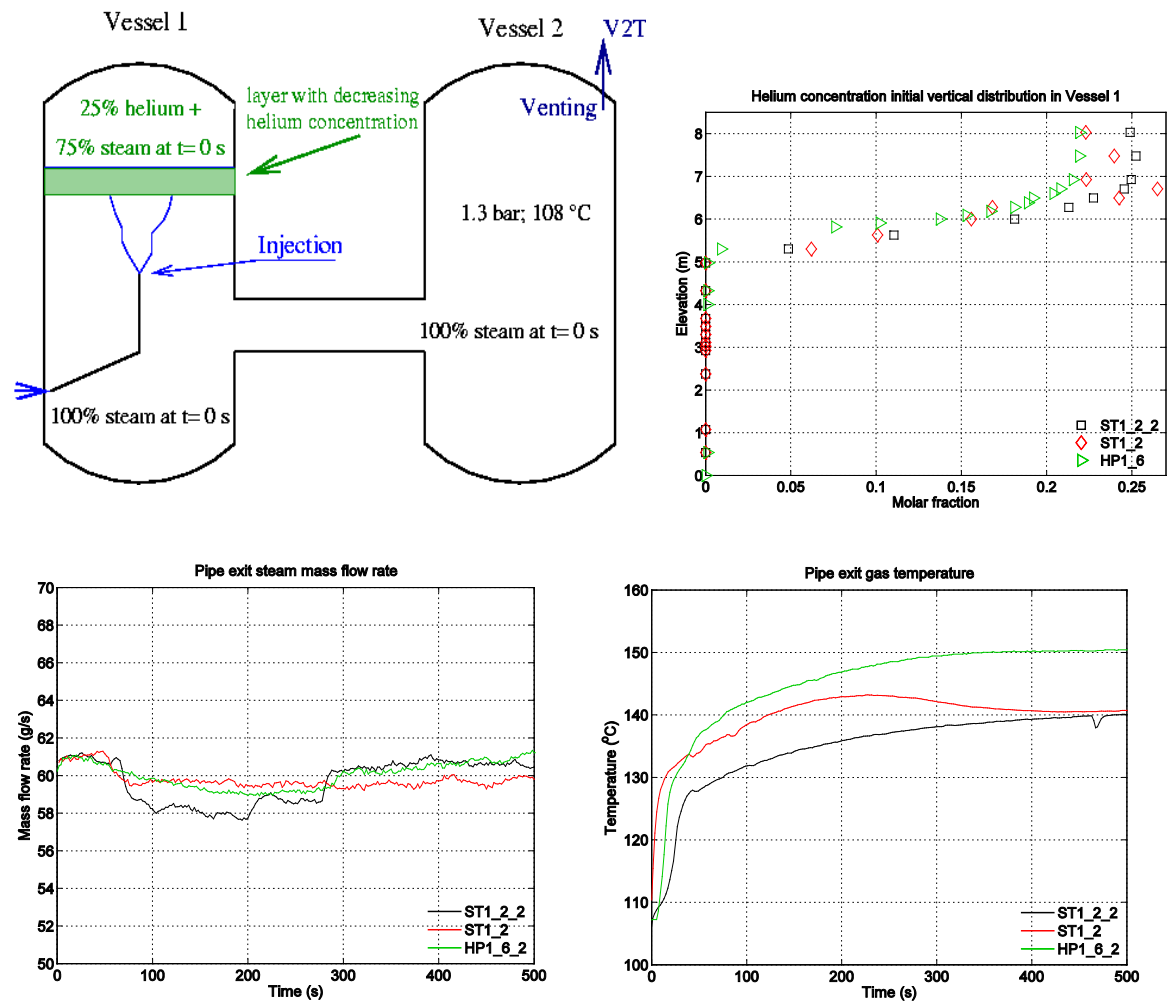

Figure 21 10 : Open benchmark, Step 5: Configuration for SETH-2 test ST1_2_2, and important initial and boundary conditions of test ST1_2 and repetition test ST1_2_2 compared with those used for Test HP1_6_2. 
1

2

3

4

5

7

8

9

10

11

12

13

14

15

16

17

18

19

20

21

22

23

24

25

26

27

28

29

30

31

32

33

34

35

36

37

38

39

40

41

42

43

44

45

46

47

48

49

50

51

52

53

54

55

56

57

58

59

60

61

62

63

64

65

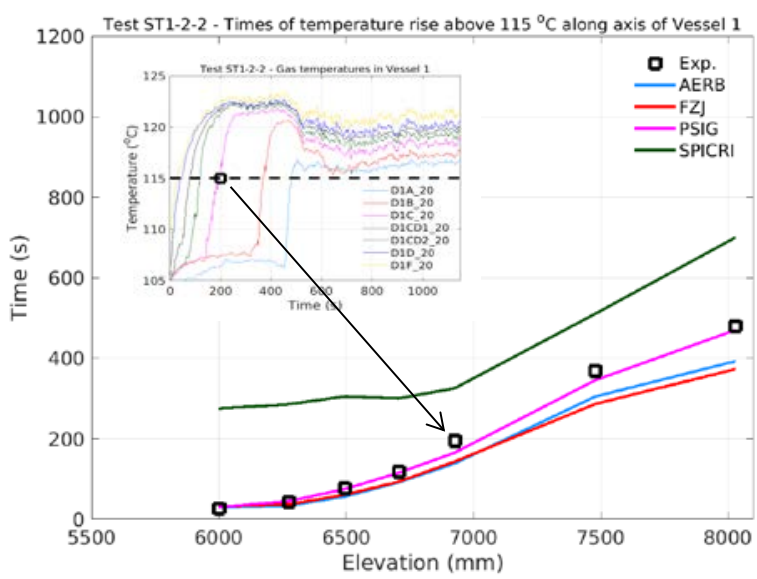

Figure 221: Open benchmark, Step 5: Progression of erosion along the axis in test ST1_2_2, using gas temperature rise times. 

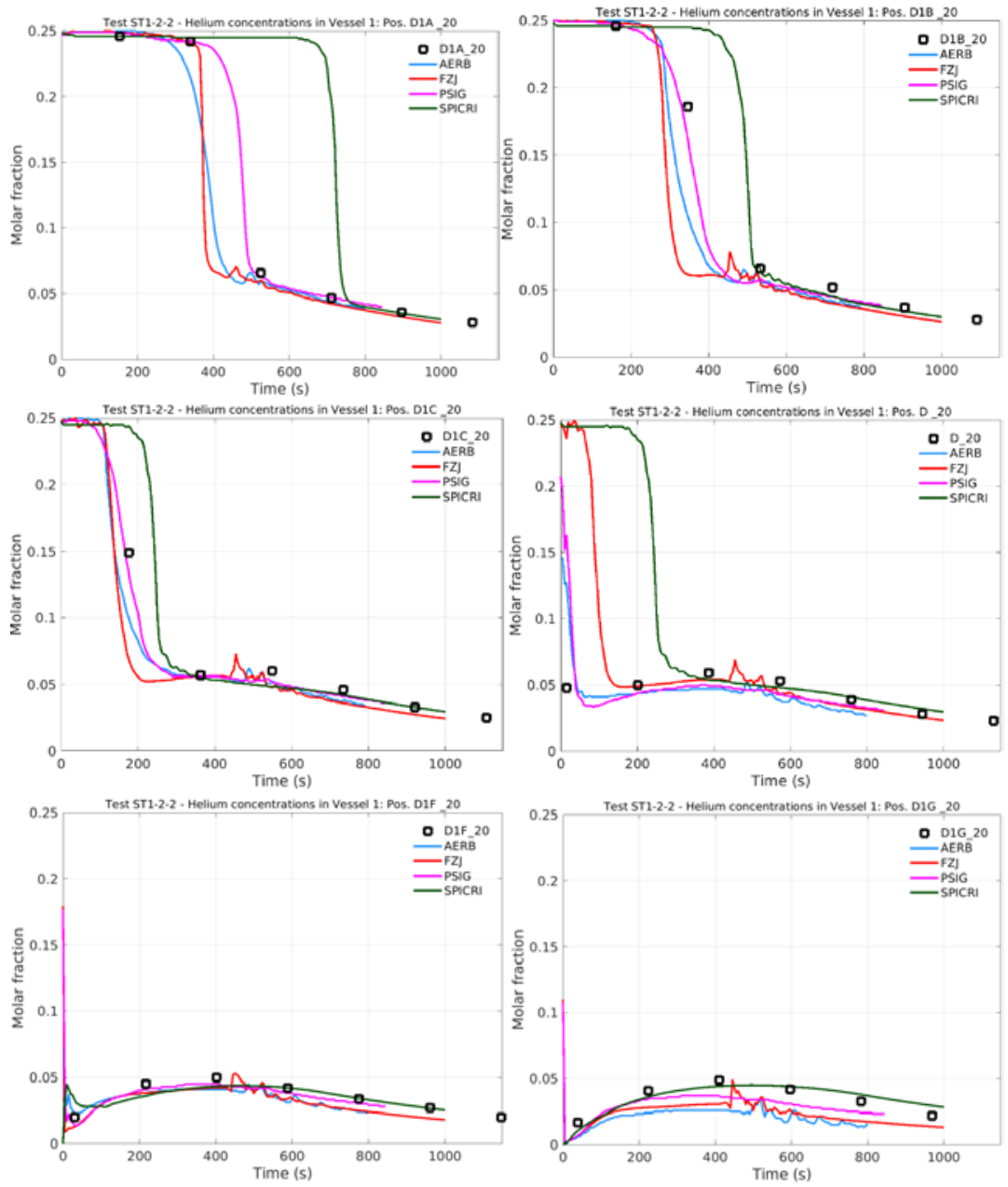

Figure 2̧z: Open benchmark, Step 5: Helium concentration evolutiontime histories at various elevations along the axis of Vessel 1. 

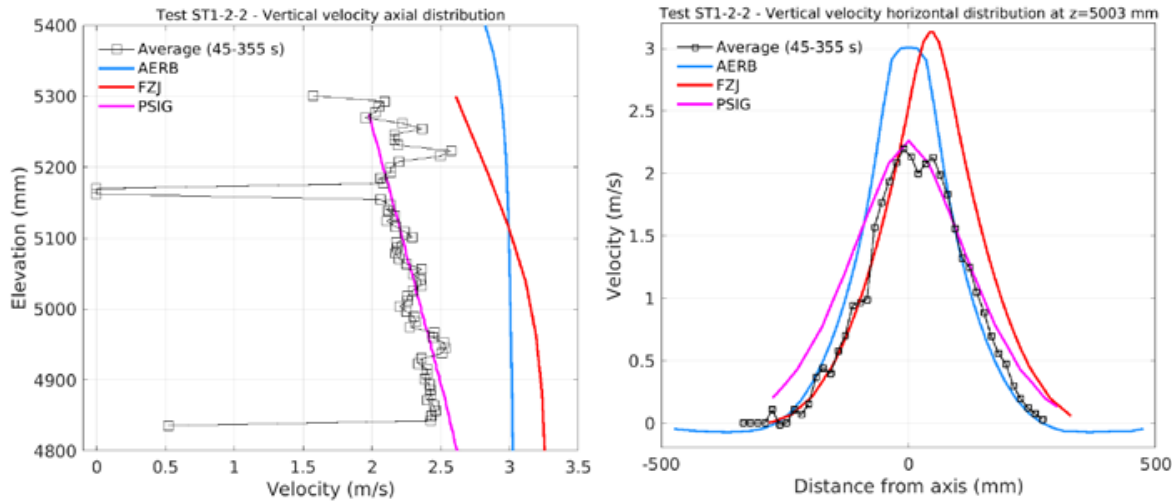

Figure 243: Open benchmark, Step 5: Vertical velocity axial profile (left) and horizontal profile at $\mathrm{z}=5003 \mathrm{~mm}$ ( $1 \mathrm{~m}$ above pipe exit)
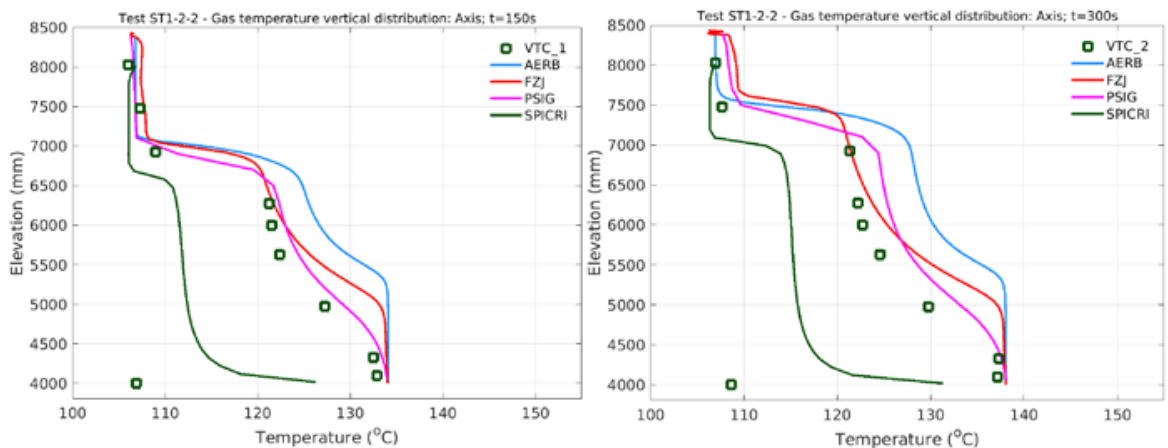

Figure 254: Open benchmark, Step 5: Axial temperature profile at two times.
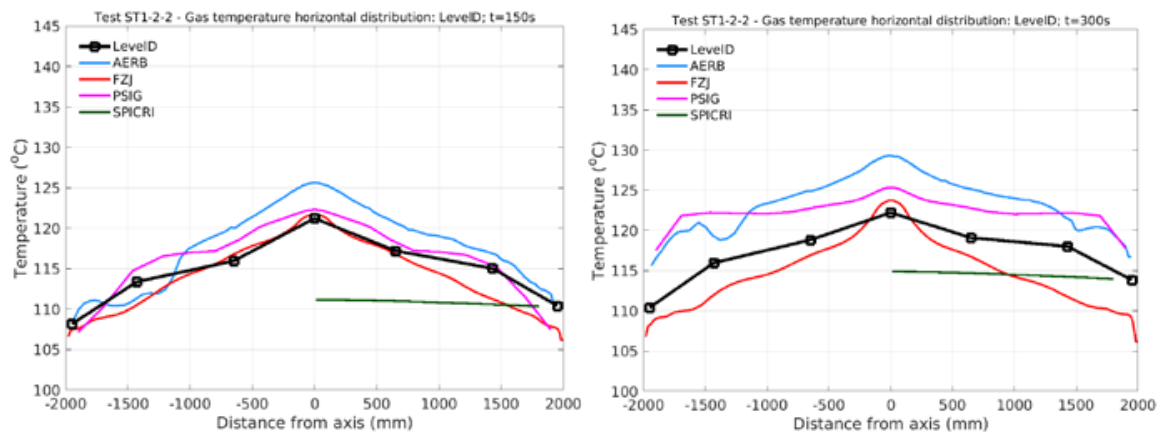

Figure 26ㄷ: Open benchmark, Step 5: Horizontal profiles at $6.3 \mathrm{~m}$ at $150 \mathrm{~s}$ (before mixing) and $300 \mathrm{~s}$ (after mixing) 

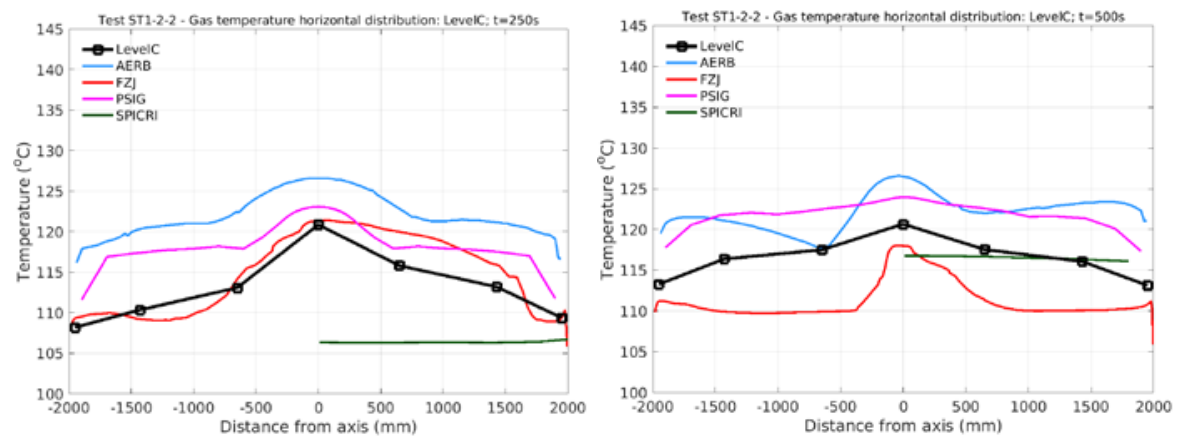

Figure 276: Open benchmark, Step 5: Horizontal profiles at $6.93 \mathrm{~m}$ at $250 \mathrm{~s}$ (during mixing or immediately after) and $500 \mathrm{~s}$ (after mixing)

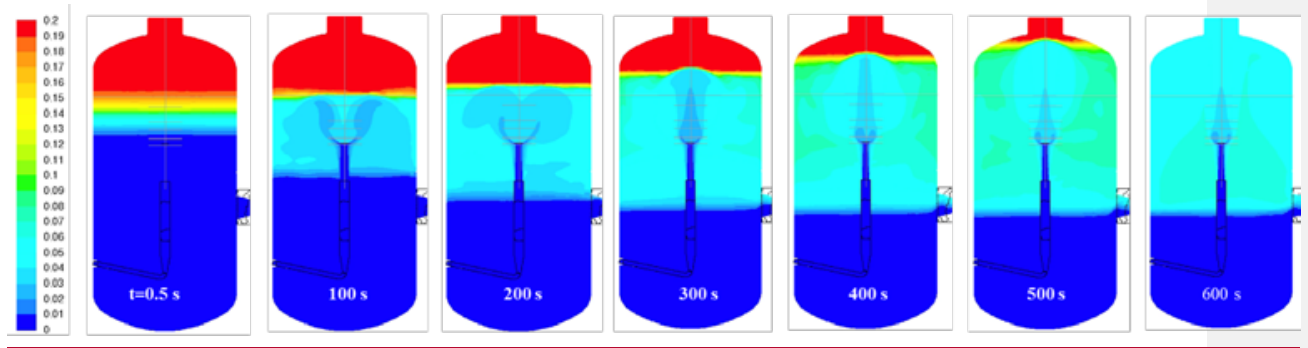

Figure 28: Open benchmark, Step 4: Calculated sequence of spatial distributions of helium concentrations at various times (contribution of VTT).
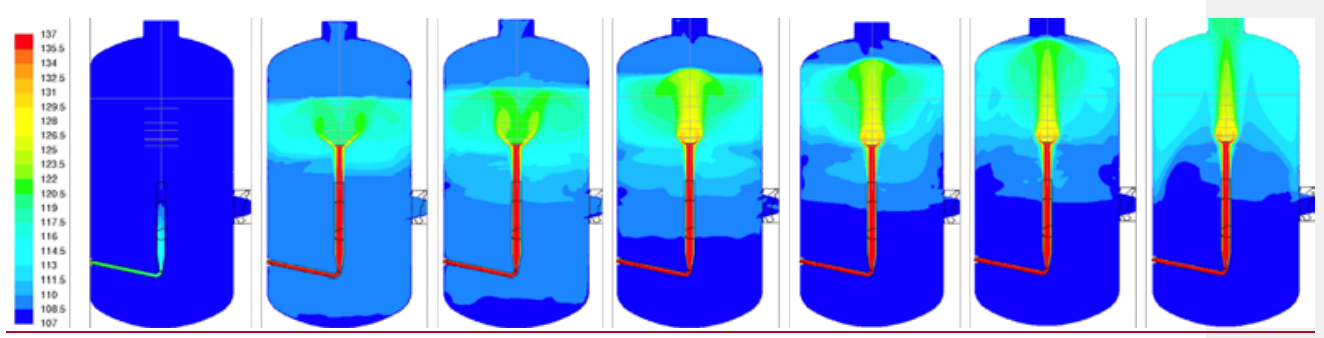

Figure 29: Open benchmark, Step 4: Calculated sequence of spatial distributions of gas temperatures at various times (contribution by VTT). 

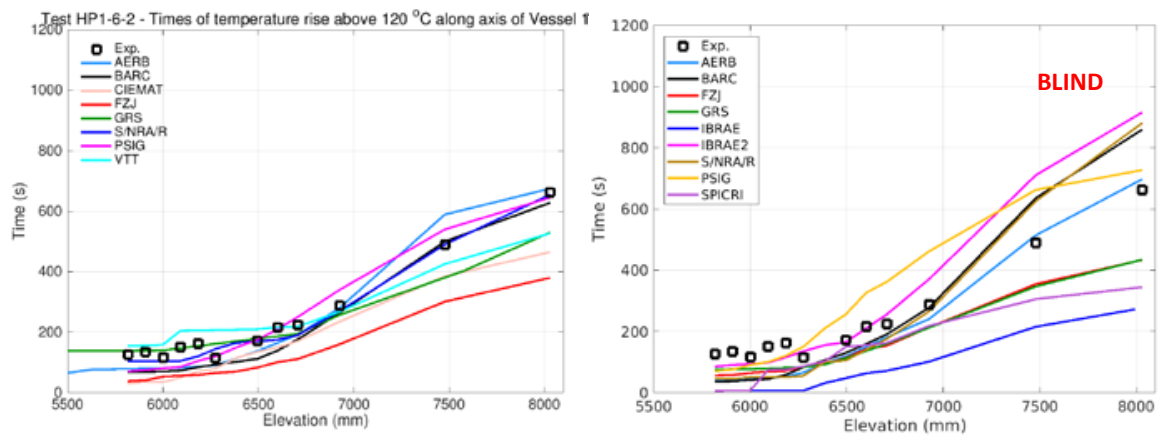

Figure 3027: Open benchmark, Step 4: Erosion progression calculated in the open phase (left), compared with best estimate results contributed to the blind benchmark.
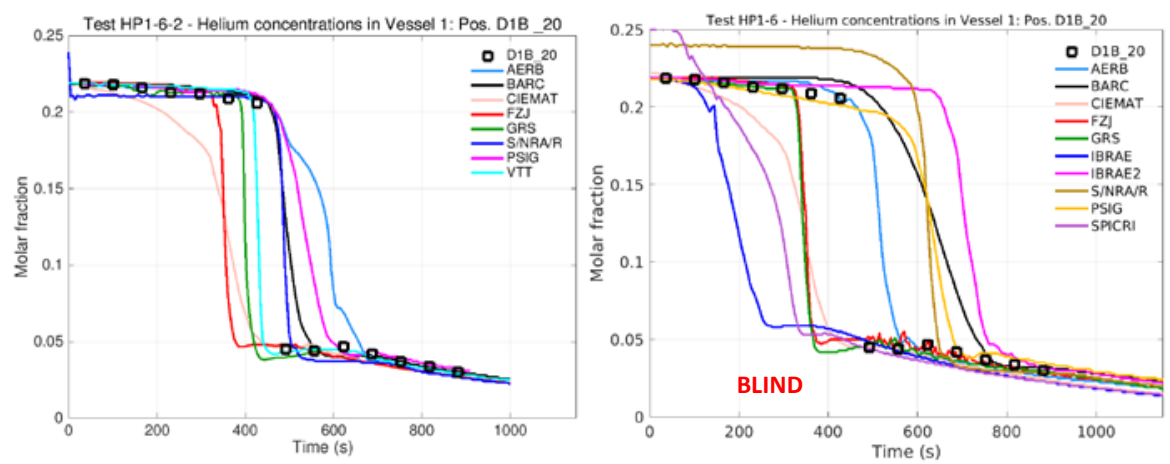

Figure 3128: Open benchmark, Step 4: Helium concentration evolutiontime history at $\mathrm{z}=6.48 \mathrm{~m}$ calculated in the open phase (left), compared with best estimate results contributed to the blind benchmark.
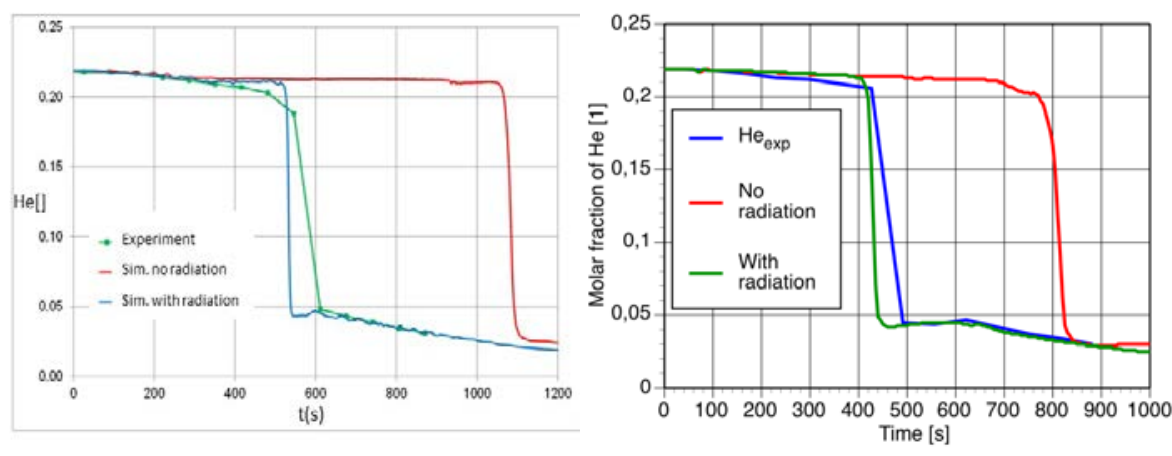
Figure 32z9: Open benchmark, Step 4: Example of results (helium concentration evolutiontime histories) obtained at $8 \mathrm{~m}$ (left) by GRS (Schramm et al., 2017) and at $7.5 \mathrm{~m}$ (right) by VTT (Huhtanen, 2018) using the model for radiation or neglecting it.
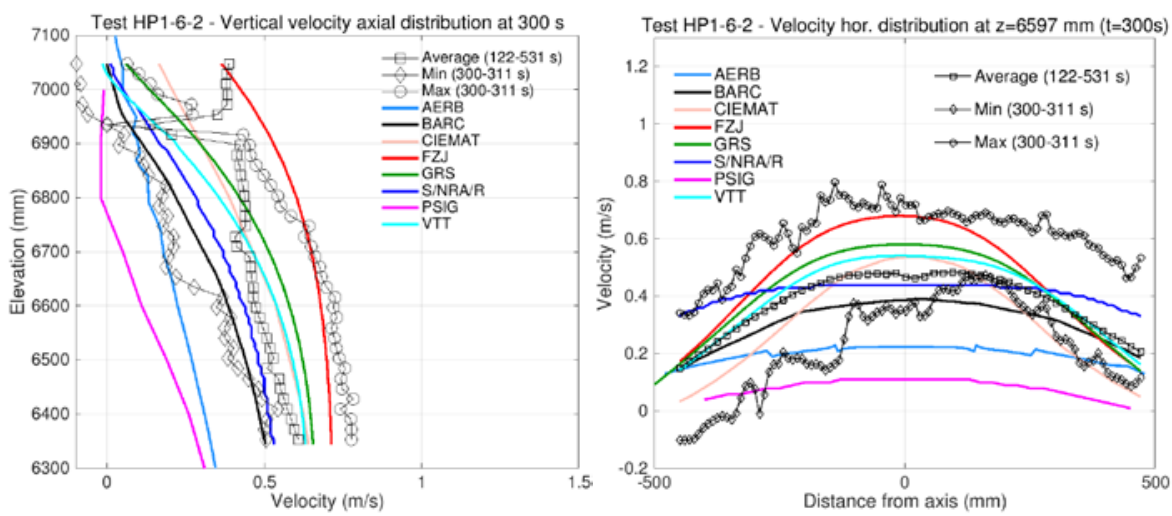

Figure 330: Open benchmark, Step 4: Vertical velocity averaged axial and horizontal profiles in the PIV window.
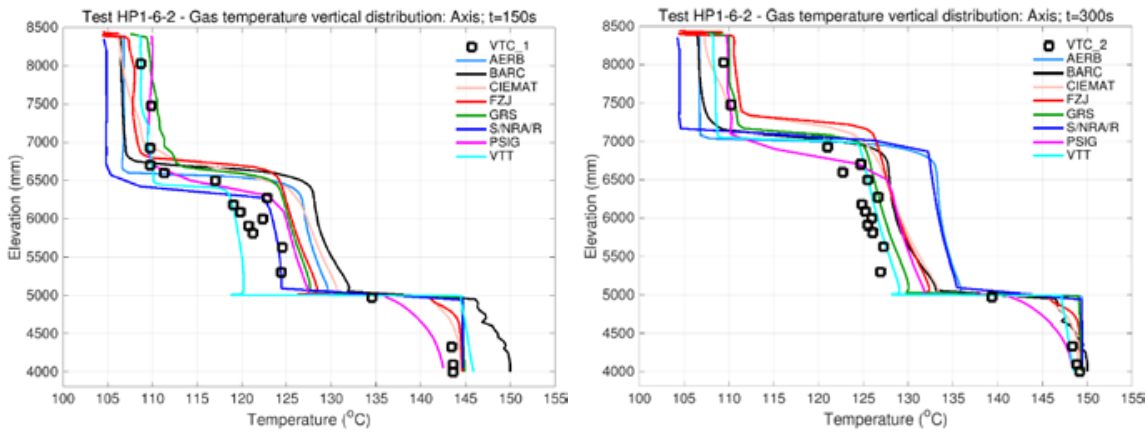

Figure 341: Open benchmark, Step 4: Axial gas temperature distributions at two times. 


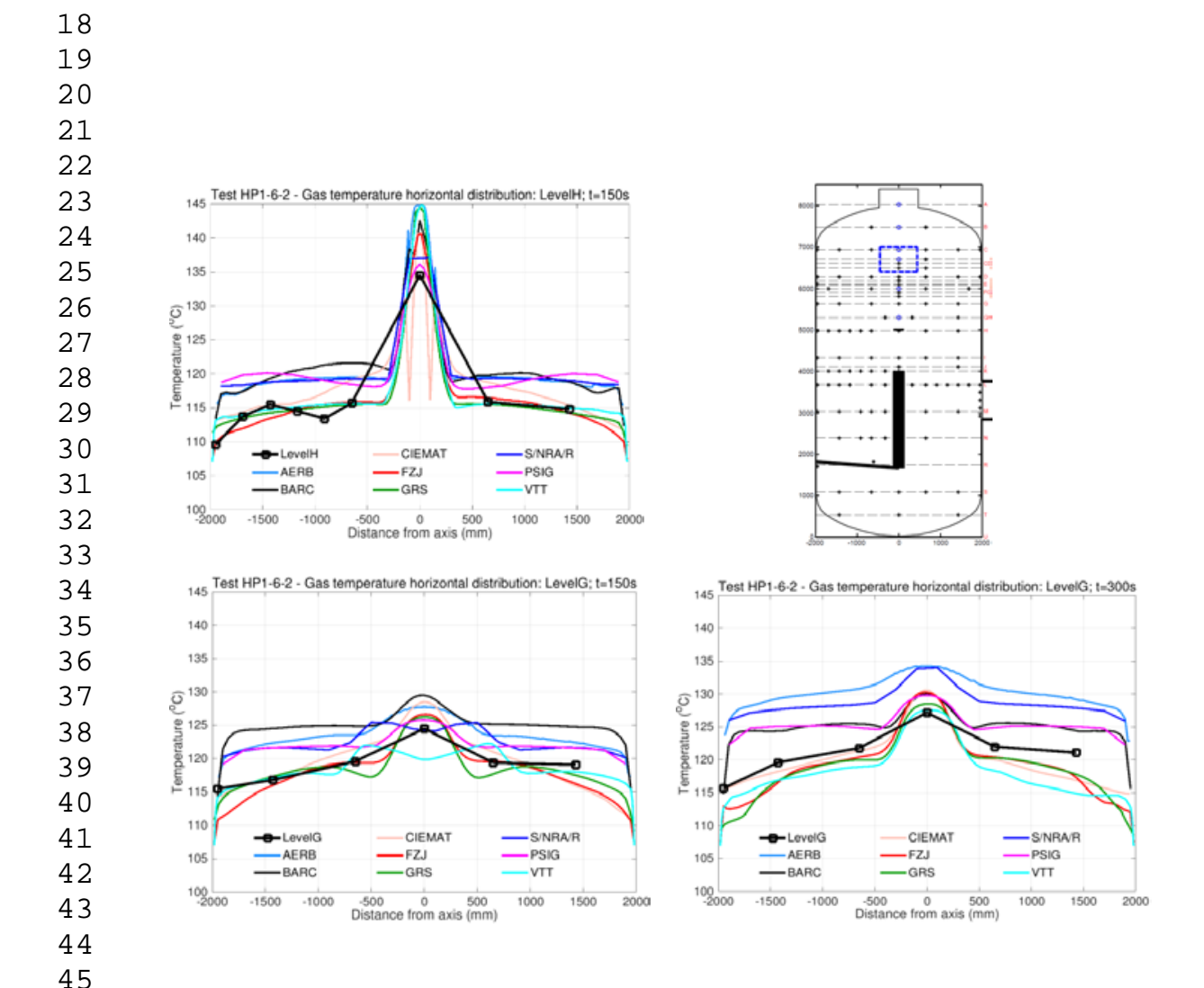



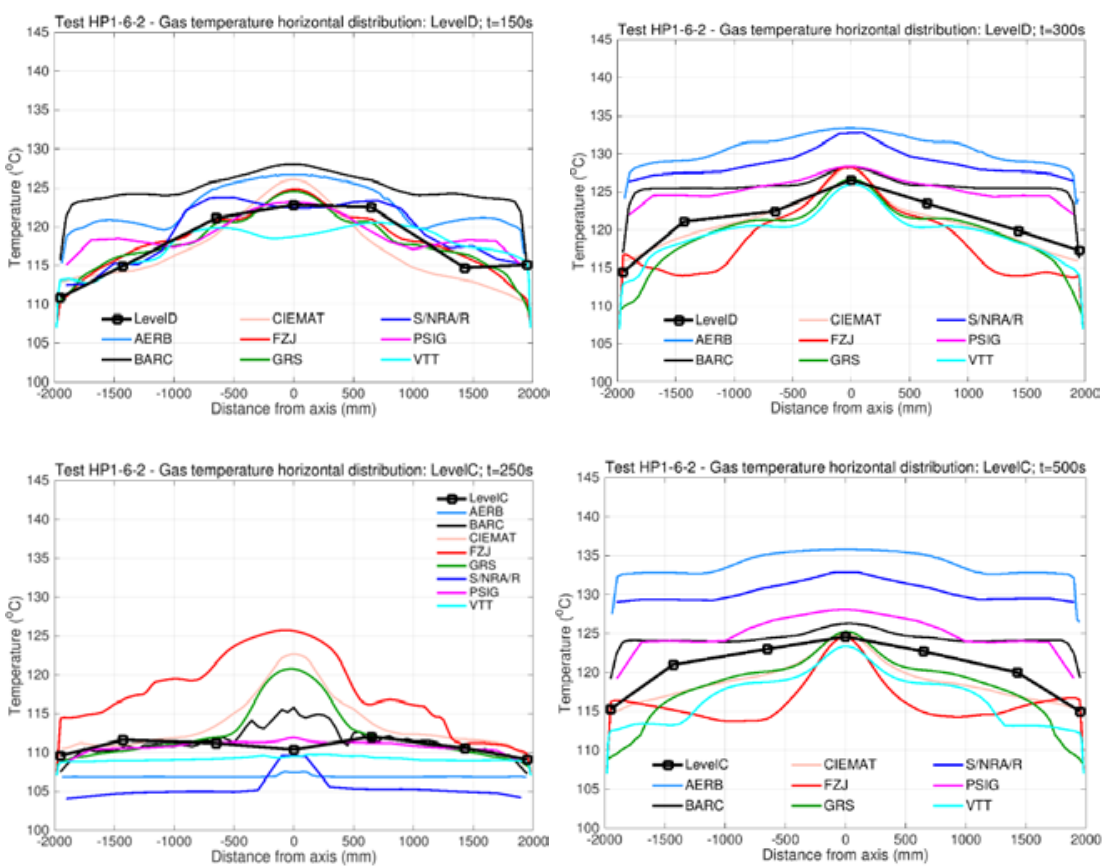

Figure 3ㅁz: Open benchmark, Step 4: Gas temperature horizontal profiles at various elevations and two times.
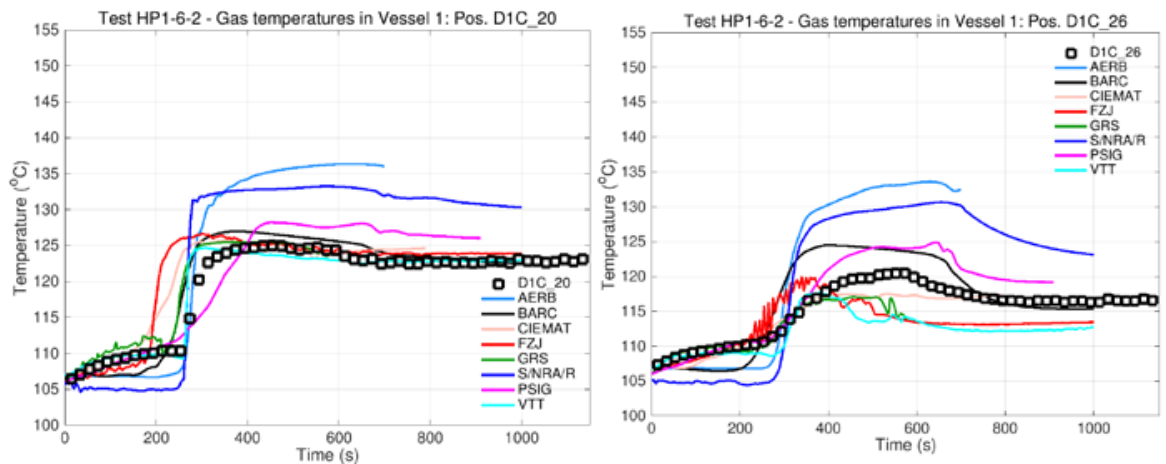

Figure 363: Open benchmark, Step 4: Gas temperature evolutiontime histories at two positions (left: on the axis; right: at $570 \mathrm{~mm}$ from the wall) at $6.5 \mathrm{~m}$. 


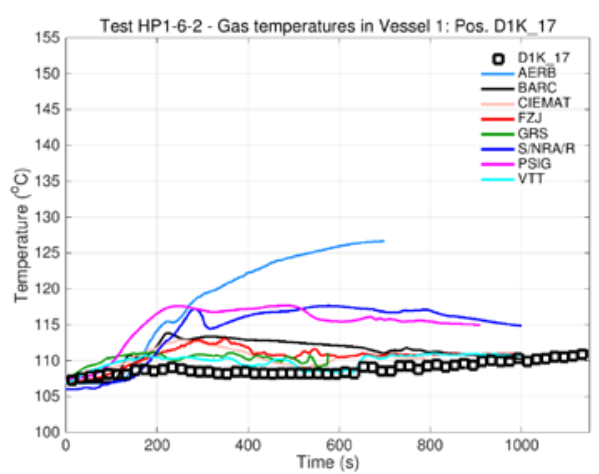

Figure 374: Open benchmark, Step 4: Off-axis ( $\mathrm{r}: \pm 325 \mathrm{~mm}$ ) gas temperature evolutiontime histories at two positons below the initial bottom of the helium layer (z: 5301 and $4000 \mathrm{~mm}$, respectively) 


\section{REFERENCES}

Abe, S., Studer, E., Ishigaki, M., Sibamoto, Y., and Yonomoto, T. (2018) “Stratification Breakup by a Diffuse Buoyant Jet: The MISTRA HM1-1 and 1-1bis Experiments and their CFD Analysis”, Nucl. Eng. Design, 331, pp. 162-175.

Abu-Romia, M.M. and Tien, C.L (1967) “Appropriate Mean Absorption Coefficients for Infrared Radiation of Gases”, Journal of Heat Transfer, 89(4), 321-327.

Allelein, H.-J., Fischer, K., Vendel, J., Malet, J., Studer, E., Schwarz, S., Houkema, M., H. Paillère, H., Bentaib, A. (2007). International Standard Problem ISP-47 on Containment Thermal Hydraulics. Nuclear Energy Agency, Committee on the Safety of Nuclear Installations, Final Report NEA/CSNI/R(2007)10, 2007.

Allein, H.-J., Reinecke, E.-A., Belt, A., Broxtermann, P., and Kelm, S. (2012) “Combined Analytical and Experimental Investigations for LWR Containment Phenomena”, Nucl. Eng, Technol., 44(3), 249260.

Andreani, M., Badillo, A., and Kapulla, R. (2016a) "Synthesis of the OECD/NEA-PSI CFD Benchmark Exercise”, Nucl. Eng. Design, 299, 59-80

Andreani, M., Daqiang, Y., Gaikwad, A.J., Ganju, S., Gera., B., Grigoryev, S., Herranz, L.E., Huhtanen, R., Kanaev, A., Kelm, S., Kim, J., Nishimura, T., Schramm, B., Sharabi, M., and Paladino, D. (2016b) "Synthesis of a blind CFD benchmark exercise based on a test in the PANDA facility addressing the stratification erosion by a vertical jet in presence of a flow obstruction”, OECD/NEA 6th Workshop on Computational Fluid Dynamics for Nuclear Reactor Safety (CFD4NRS-6), MIT, Cambridge, MA-USA, 13-15 September, 2016.

Andreani, M. and Paladino D., (Benchmark Report, 2018), prepared by PSI (with appendices contributed by AERB, FZJ and VTT) (2018) “OECD/NEA HYMERES project: synthesis of results of the benchmark on PANDA test HP1_6_2”, PSI Technical Note AN-42-17-07 Rev.1, Project report HYMERES-P-17-48, May 2018. 
Bentaib, A., Meynet, N., and Bleyer, A. (2015) “Overview on Hydrogen Risk Research and Development Activities: Methodology and Open Issues”, Nucl. Eng. Technol., 47, 26-32.

Breitung, W. and Royl, P. (2000) "Procedure and Tools for Deterministic Analysis and Control of Hydrogen Behavior in Severe Accidents”. Nucl. Eng. Design, 202, 249-268.

Chan, C.K. and Jones, S.C. (1997) “Gas Mixing Experiments in a Large Enclosure”, Proc. of the $18^{\text {th }}$ Annual Conf. of the Canadian Nuclear Society

Choi, Y.-S., Lee, U.-J., and Park, G.-C. (2001) “Study on local hydrogen behaviors in a subcompartment of the NPP containment”, Nucl. Eng, Design, 208, 99-116.

Deri, E., Cariteau, B., and Abdo D. (2010) “Air fountains in the erosion of gaseous stratifications”, Int. J. Heat and Fluid Flow, Volume 31, Issue 5, Pages 935-941.

Erkan, N., Mignot, G., Kapulla, R., Paladino, D., Zboray, R., Strassberger, H.J., Bissels, W. and Fehlmann, M. (2009) “OECD SETH-2 PANDA Test ST1_2_2 Quick-Look Report”, PSI internal technical report TM-42-09-07-0, April 2009.

Filippov, A.S., Grigoryev, S.Yu., O.V. Tarasov, O.V. (2017) “On the possible role of thermal radiation in containment thermal-hydraulics experiments by the example of CFD analysis of TOSQAN T114 airHe test”, Nucl. Eng. Design, 310, 175-186.

Gallego E., Migoya E., Martín-Valdepeñas, J.M., Crespo, A.,García, J., Venetsanos, A. Papanikolaou, E., Kumar, S., Studer, E., Dagba, Y., Jordan ,T., Jahn, W., Høiset, S., Makarov, D., Piechna, J., (2007) “An intercomparison exercise on the capabilities of CFD models to predict distribution and mixing of $\mathrm{H}_{2}$ in a closed vessel”, International Journal of Hydrogen Energy, 32, 2235 $-2245$

Gupta, S. (2015) “Experimental Investigations Relevant for Hydrogen and Fission Product Issues Raised by the Fukushima Accident”, Nucl. Eng. Technol., 47, 11-25.

Howell. J.R. (1988) “Thermal Radiation in Participating Media: The Past the Present, and Some Possible Futures”, Journal Heat Transfer, 110, 1220-1229.

Huhtanen, R. (2018), Private Communication. 
Kapulla, R., Mignot, G., Paranjape, S., Suter, S., Fehlmann, M., and Paladino, D. (2015a) “OECD/NEA HYMERES Project: Jet/Plume interacting with flow obstruction HP1 Series. Test Series Report”, PSI internal report TM-42-15-16, Rev.0, Project report HYMERES-P-15-26, Nov. 2015 (report restricted to project participants).

Kapulla, R., Paranjape, S., Mignot, G., Suter, S., Fehlmann, M., and Paladino D. (2015b) “OECD/NEA HYMERES Project: PANDA Tests HP1_6_0, HP1_7_0, HP1_8_0, and HP1_678_Disk Data Report”, PSI internal report TM-42-15-13 Rev. 0, Project report HYMERES-P-15-25, October 2015 (report restricted to project participants).

Karwat, H. et al. (1999) "State-of-the-Art Report on Containment Thermal-hydraulics and Hydrogen Distribution”, OECD/NEA group of experts, CSNI/R(99)-16 (1999).

Kelm, S., Ritterath, M., Prasser, H.-M., and Allelein, H.J. (2016a) "Application of the MINIPANDA Test Case ‘Erosion of a Stratified Layer by a Vertical Jet’ for CFD Validation”, Nucl. Eng. Design, 299, 124-135.

Kelm, S., Müller, H., and Allelein, H.-J. (2106b) "Importance of thermal radiation heat transfer modeling in containment typical flows”, Paper submitted for presentation at the CFD4NRS-6, MIT, Cambridge, MA, USA, September 13-15.

Liang, R. et al., (2015) "Status Report on Hydrogen Management and Related Computer Codes“, NEA/CSNI/R(2014)8,

Lopez-Alonso E., Papini D., and Jimenez G. (2017) “Hydrogen Distribution and Passive Autocatalytic Recombiner (PAR) Mitigation in a PWR-KWU Containment Type”, Annals of Nuclear Energy, 109, pp. 600-611.

Mahaffy, J., et al. (2015). Best Practice Guidelines for the Use of CFD in Nuclear Reactor Safety Applications - Revision” NEA/CSNI/R(2014)11.

Noutsopoulos, G.C. and Yannopoulos, P.C. (1989) “Axial Dilution in Obstructed Round Buoyant Jet”, Journal of Hydraulic Engineering, 115(1), pp. 71-81. 
Nishimura, T., Hoshi, H. and Hotta, A. (2015) “Current Research and Development Activities on Fission Products and Hydrogen Risk after the Accident at Fukushima Daiichi Nuclear Power Station”, Nucl. Eng. Technol., 47, 1-10

OECD/NEA HYMERES-2 project (2017-2021).

OECD/NEA THAI Project (2010) "Hydrogen and Fission Product Issues Relevant for Containment Safety Assessment under Severe Accident Conditions” Final Report, 22 June 2010, Report NEA/CSNI/R(2010)3.

Paladino, D. and Dreier, J. (2012) “PANDA a Multi Purposes Integral Test Facility”, Science and Technology of Nuclear Installations, 2012, Article ID 239319, doi:10.1155/2012/239319.

Paladino, D., Mignot, G., Kapulla, R., Zboray, R., Andreani, M., Tkatschenko, I., Studer, E., and Brinster, J. (2013) “OECD/SETH-2 Project: PANDA and MISTRA Experiments addressing Key Safety Issues for Water Reactor Containment”, Proceedings of the $15^{\text {th }}$ International Topical Meeting on Nuclear Reactor Thermal-hydraulics (NURETH-15), paper 106, Pisa, Italy, 12-17 May 12-17, 2013.

Paladino, D., Andreani, M., Guentay, S., Mignot, G., Kapulla, R., Paranjape, S., Sharabi, M., Kisselev, A.,Yudina, T., Filippov, Kamnev, M., Khizbullin, A., Tyurikov, O., Liang, Z., Daniele Abdo, D., Brinster, J., Dabbene, F., Kelm, S., Klauck, M., Götz, L., Gehr, R., Malet, J., Bentaib, A., Bleyer, A., Lemaitre, P., Porcheron, E., Benz, S., Jordan, T., Xu, Z., Boyd, C., Siccama, A., Visser, D. (2016) "Outcomes from the EURATOM-ROSATOM ERCOSAM SAMARA projects on containment thermal-hydraulics for severe accident management”, Nuclear Eng. and Design, 308, 103-114.

Paranjape, S., Kapulla, R., Mignot, G. and Paladino D. (2018) “Gas Redistribution Caused by Interacting Heat Sources in the Presence of a Vertical Condenser," $12^{\text {th }}$ International Topical Meeting on Nuclear Reactor Thermal-Hydraulics, Operation and Safety (NUTHOS-12), Qingdao, China, October 14-18, 2018.

Sarikurt, F.S. and Hassan, Y.A. (2017) "Large Eddy Simulations of Erosion of a Stratified Layer by a Buoyant Jet”, Int. J. of Heat and Mass Transfer, Vol. 112, p. 354-365. 
Schramm, B., Stewering, J., and Sonnenkalb M. (2017) “Einsatz von CFD-Codes für die Simulation von unfalltypischen Phänomenen im Sicherheitseinschluss: Validierung und gezielte Modellerweiterung”, Anschlussbericht RS1526, 2017 GRS-472, ISBN 978-3-946607-55-7.

Schwarz, S., Fischer, K., Bentaib, A., Burkhardt, J., Lee, J.-J., Duspiva, J., Visser, D., Kyttala, J., Royl, P., Kim, J., Kostka, P. and Liang, R. (2011) “Benchmark on Hydrogen Distribution in a Containment based on the OECD-NEA THAI HM-2 Experiment”, Nucl. Technol., 175(3), 594-603. Smith B.L. (2009) “Identification and Prioritization of Generic Nuclear Safety Problems Requiring CFD Analysis”, Proc. 17 Int. Conf. on Nuclear Engineering (ICONE-17), Paper 75482, Brussels, Belgium, July12-16, 2009.

Studer, E., Brinster, J., Tkatschenko, I., Mignot, G., Paladino, D., and Andreani, M. (2012)

"Interaction of a light gas stratified layer with an air jet coming from below: Large scale experiments and scaling issues”, Nucl. Eng. Design, 253, 406-412.

Visser, D.C., Houkema, M., Siccama, N.B., and Komen, E.M.J. (2012) "Validation of a FLUENT CFD model for hydrogen distribution in a containment”, Nuclear Engineering and Design, 245, 161171. 